\title{
ONCOSTATIN M PROMOTES BREAST CANCER METASTASIS: INCREASED EXPRESSION OF PRO-ANGIOGENIC FACTORS, INFLAMMATORY CYTOKINE EXPRESSION, AND CIRCULATING TUMOR CELL NUMBERS
}

by

Ken Tawara

\author{
A dissertation \\ submitted in partial fulfillment \\ of the requirements for the degree of \\ Doctor of Philosophy in Biomolecular Sciences \\ Boise State University
}

December 2017 
(C) 2017

Ken Tawara

ALL RIGHTS RESERVED 


\section{BOISE STATE UNIVERSITY GRADUATE COLLEGE}

\section{DEFENSE COMMITTEE AND FINAL READING APPROVALS}

of the dissertation submitted by

Ken Tawara

Dissertation Title: Oncostatin M Promotes Breast Cancer Metastasis: Increased Expression of Pro-Angiogenic Factors, Inflammatory Cytokine Expression, and Circulating Tumor Cell Numbers

Date of Final Oral Examination: $\quad 25$ August 2017

The following individuals read and discussed the dissertation submitted by student Ken Tawara, and they evaluated his presentation and response to questions during the final oral examination. They found that the student passed the final oral examination.

Cheryl Jorcyk, Ph.D. Chair, Supervisory Committee

Kenneth A. Cornell, Ph.D. Member, Supervisory Committee

Daniel Fologea, Ph.D. Member, Supervisory Committee

Denise G. Wingett, Ph.D. Member, Supervisory Committee

The final reading approval of the dissertation was granted by Cheryl Jorcyk, Ph.D., Chair of the Supervisory Committee. The dissertation was approved by the Graduate College. 


\section{ACKNOWLEDGEMENTS}

I would like to thank my dissertation advisor and mentor, Dr. Cheryl Jorcyk, who has helped me grow as a scientist through my masters and doctoral studies. I am extremely grateful for her honest feedback and critical assessment on my ideas, writing, project directions, and for always pushing me to do the best that I can do. Her unwavering belief in my success has helped me drive myself to be much more productive as a graduate student than I could have ever imagined. I would also like to thank the Department of Biological Sciences, the Biomolecular Sciences Graduate Program (BMOL), the BMOL Program Coordinator, Beth Gee, and the Program Director, Dr. Denise Wingett, for their support of the advancement of my research. They have given me the opportunity to develop skills as a scientist within and beyond the laboratory.

I would also like to thank the many people with whom I have collaborated and worked with in the lab. In particular, it was a pleasure to work to Dr. Celeste Bolin. Her expertise in animal experimentation and writing techniques have helped me tremendously to further my skills as a scientist. I would also like to thank her for the invitation to speak at the Biology Colloquium at College of Idaho, which has given me invaluable public speaking experience. I would also like to thank Dr. Julie Oxford, Dr. Randall Ryan, Dr. Caleb Sutherland, and now Dr. Hunter Covert for working with me on multiple publications and the opportunity to work on projects outside my main field of expertise. Furthermore, I would like to thank Dr. William Hughes and the Keck Foundation for 
supporting me and helping me gain invaluable experience in managing human subject protocols with the Institutional Review Board.

I would also like to thank numerous past and present members in the lab that have helped me on my numerous projects. Specifically, I would like to thank Ryan Fox, Dollie LaJoie, Danielle Hedeen, Madhuri Nandakumar, M.S. Jake Goyden, M.S. Jordan Koncinsky, Dr. Jeff Redshaw, Dr. Patrick Aranda, Cody Wolf, Hannah Scott, and Jaqueline Emathinger. They have helped me on various aspects of my projects from assisting me on a few assays in the lab to helping develop new techniques and protocols. I would also like to thank our lab, the Laboratory of Inflammatory Cytokines in Tumor Invasion and Metastasis. Working in the lab has helped me not only to manage multiple projects but also to navigate the various regulations and paper work associated with institutional review boards. Furthermore, it has helped me gain experience in grant writing, budgets, and the complex nature of managing laboratory supplies. Perhaps most importantly, it has helped me gain valuable skills to improve my scientific writing and oral communication skills. Finally, I would also like to thank my committee members Dr. Denise Wingett, Dr. Ken Cornell, and Dr. Daniel Fologea for their valuable input and insight into my projects. Their feedback has helped me narrow down my focus to parts of my projects that were the most interesting with highest probability of success. 


\begin{abstract}
Breast cancer is the most diagnosed cancer type in women and its resultant mortality is second only to lung cancer worldwide. While breast cancer is known to have many risk factors, inflammation remains an unquantifiable risk, and it can arise from obesity, depression, poor health, autoimmune diseases, and other conditions that cause systemic chronic inflammation. Chronic inflammation is gaining recognition for its role in cancer development, the potentiation of a metastatic phenotype in cancer cells, and decreased survival in breast cancer patients. In particular, inflammatory cytokines in the interleukin-6 (IL-6) family have been shown to promote an epithelial-mesenchymal transition (EMT), tumor cell detachment, invasion, and metastasis. However, therapies to inhibit IL-6 have not been successful in treating solid tumors. This is most likely due to redundancy, as there are other inflammatory cytokines such as oncostatin M (OSM) and interleukin-1 beta (IL-1 $\beta$ ) that demonstrate overlapping effects in cancer progression. In these studies, the interactions between OSM, IL- 6 and IL-1 $\beta$ were addressed. First, OSM and IL-6 were shown to induce vascular endothelial growth factor (VEGF) in a breast cancer subtype-specific manner. Next, OSM was assessed for its capacity to increase circulating tumor cell numbers in mouse models of human breast cancer. Lastly, OSM, IL-6, and IL-1 $\beta$ expression levels were shown to correlate with each other in breast cancer, and high co-expression of these cytokines was shown to lead to decreased patient survival. Furthermore, OSM was assessed for its synergistic relationship with IL-1 $\beta$ in inducing IL-6 secretion from breast cancer cells. Together, these results suggest that
\end{abstract}


inflammatory cytokines promote metastatic disease in a breast cancer subtype-dependent manner. Importantly, these studies both provide a rationale for the development of breast cancer therapeutic regimens that target multiple cytokines as well as help explain why single anti-cytokine therapies have failed in clinical trials. 
TABLE OF CONTENTS

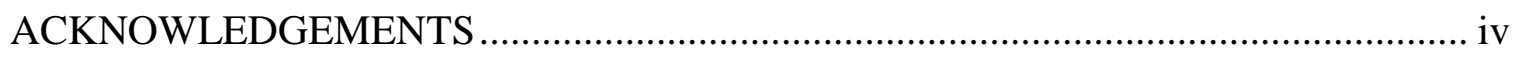

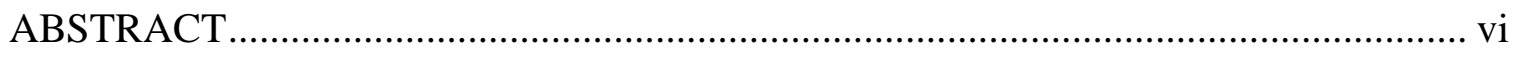

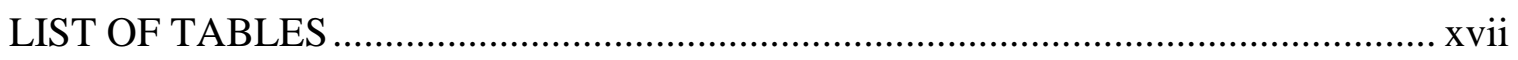

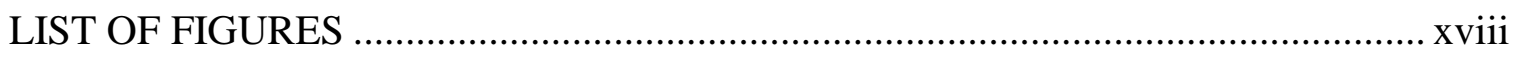

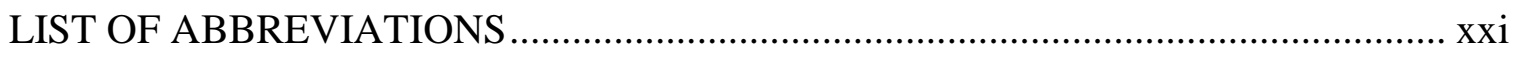

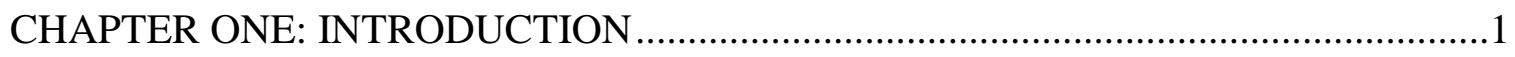

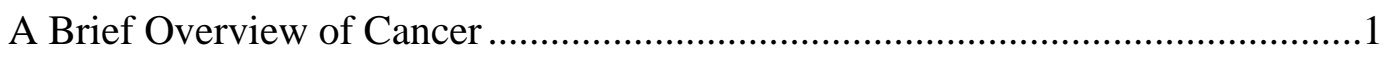

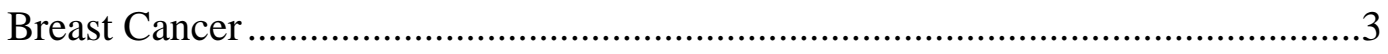

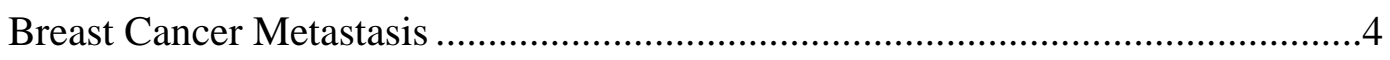

Inflammation in Breast Cancer .....................................................................6

Breast Cancer Subtypes ...........................................................................

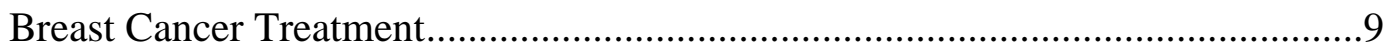

Intracellular Pathways That Promote Breast Cancer Progression ..........................10

Oncostatin M and Breast Cancer .....................................................................11

Interleukin-6, And Interleukin-1 Beta in Breast Cancer.......................................13

Cancer Angiogenesis and Hypoxia Factors ………….........................................14

Circulating Tumor Cells and Their Detection ....................................................15

Mouse Models for Breast Cancer ........................................................................17

Human Data Analysis ................................................................................18 
Summary

Figures

Figure 1.1 Chronic inflammation leads to genomic and epigenetic instability and promotes tumor formation.

Figure 1.2. Multi-step development of invasive ductal carcinoma (IDC). 24

Figure 1.3. Treatment strategies for breast cancer subtypes .... .25

Figure 1.4. Multiple signaling pathway activation in breast cancer cells. .26

Figure 1.5. OSM induces the STAT3, PI3K, and MAPK pathways. ........27

Figure 1.6. Tumor cells promote the development of angiogenesis.........28

References

CHAPTER TWO: CO-EXPRESSION OF VEGF AND IL-6 FAMILY CYTOKINES

ARE ASSOCIATED WITH DECREASED SURVIVAL IN HER2- BREAST CANCER:

SUBTYPE SPECIFIC IL-6 FAMILY CYTOKINE-MEDIATED VEGF SECRETION .45

Authors .45

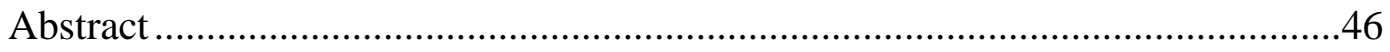

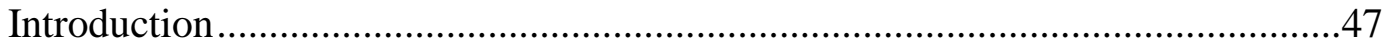

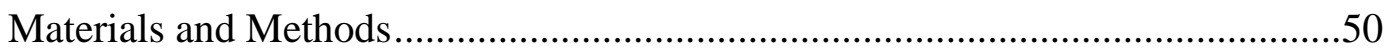

Oncomine analysis ...................................................................

Tissue culture .........................................................................

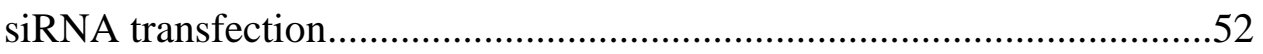

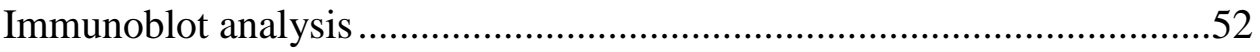

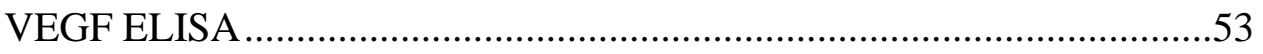

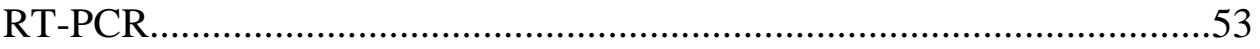

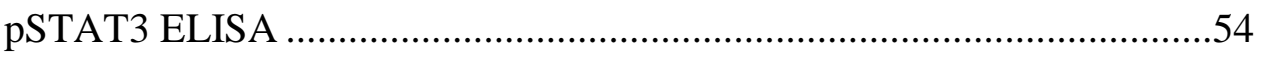

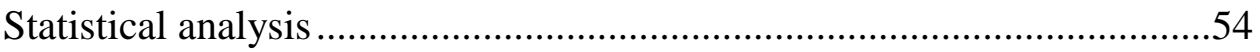


Results.

Inflammatory cytokine and VEGF co-expression is correlated with decreased survival of IDC patients

HER2- status dictates poor survival in IDC patients with high coexpression of OSM, IL-6, OSMR $\beta$ or IL-6R $\alpha$ and VEGF.

OSM mediates VEGF induction independent of HIF1 $\alpha$ signaling in TNBC cells

OSM strongly induces STAT3 activation in ER+ and ER- HER2- breast cancer cells.....

Activation of STAT3 signaling is required for VEGF secretion by MDAMB-231 TNBC cells

Discussion .60

Conclusions .64

Figure 2.1. Inflammatory cytokine and VEGF co-expression are correlated with decreased survival of invasive ductal carcinoma patient.

Figure 2.2 Co-expression of VEGF with OSM or OSMR $\beta$ appears to affect survival in HER2- but not HER2+ subtypes

Figure 2.3. IL-6 family cytokine OSM induces VEGF secretion independent of HIF1 $\alpha$ signaling in MDA-MB-231 cells. .70

Figure 2.4. OSM strongly induces phosphorylation of STAT3 .72

Figure 2.5. OSM induces VEGF via STAT3 signaling. .73

Figure 2.6. Mechanistically distinct regulation of VEGF secretion in MDA-MB-231 TNBC and T47D (ER+/PR+/HER2-) cells.

Supplemental Materials and Methods .75

Endothelial Cell Tube and Branch Point Formation Assay..... .75

In vivo Matrigel Plug Assay. .75

JNK Inhibitor Assay .76 
Chapter Two: Supplemental Figures

Figure 2.S1. Increased co-expression of OSM or OSMR $\beta$ and VEGF correlates with decreased survival of IDC patients......................................77

Figure 2.S2. Expression of cytokines or their receptors and VEGF appears to affect survival in HER2- but not HER2+ subtypes................................79

Figure 2.S3. Co-expression of OSM or OSMR $\beta$ and VEGF differentially affects survival based on estrogen receptor status. .81

Figure 2.S4. OSM induces endothelial cell tube formation and branch points in vitro.

Figure 2.S5. OSM-treatment of human breast cancer cells stimulates angiogenesis in vivo.

Figure 2.S6. JNK Inhibitor 2 suppresses VEGF secretion and phosphorylation of STAT3.

References

\section{CHAPTER THREE: ONCOSTATIN M PROMOTES BREAST CANCER METASTASIS TO LUNG AND INCREASES CIRCULATING TUMOR CELL} NUMBER

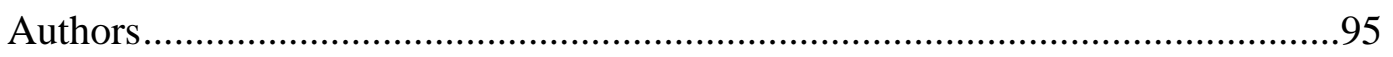

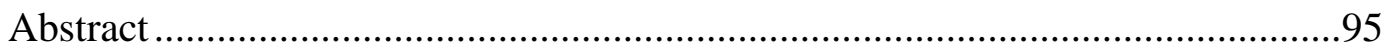

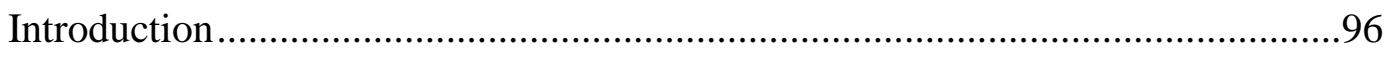

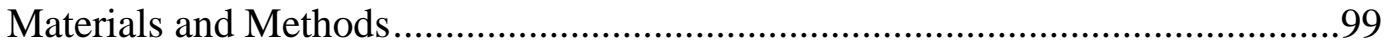

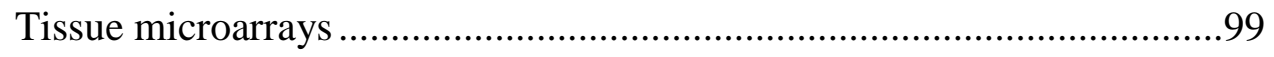

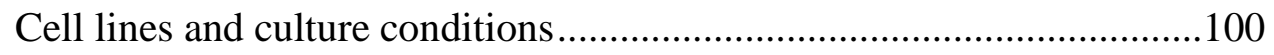

Plasmid construct design and cell transfections.......................................100

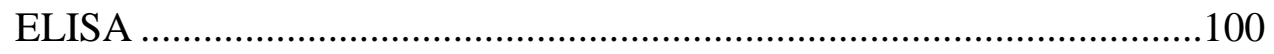

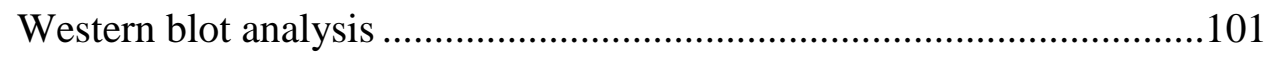

Animals and tumor cell injections ...........................................................101 
In vivo bioluminescence imaging and tumor progression. .103

Detection of circulating tumor cells (Alu qPCR) 103

Quantitative PCR (qPCR). 104

In-vivo magnetic resonance imaging 104

Detection of circulating tumor cells (clonogenic assay)

Epithelial-mesenchymal transition assay 106

Cell migration assay. 106

Cell detachment assay 106

Statistical analysis

Results .107

High OSM expression in breast ductal carcinoma in situ (DCIS) and IDC suggests autocrine signaling

OSM generated from TET-inducible MDA-MB-231 $\left(\mathrm{MDA}^{\mathrm{TO} / \mathrm{OSM}}\right)$ cells increases metastases to the lung and decreases survival.

OSM increases metastasis to lung and circulating tumor cell numbers in an orthotopic MDA-MB-231 model of breast cancer.

Suppression of OSM in syngeneic mouse model reduced lung metastases

Suppression of OSM by shRNA increases survival from spontaneous metastasis via orthotopic injection but not via intracardiac injection in vivo

CTC number and metastatic burden is reduced in OSM knockout mice compared to wild type mice

OSM increases pre-intravasation metrics of metastatic capacity in 4T1.2 cells

Discussion

Conclusions 118 
Declarations 118

Chapter Three: Figures

Figure 3.1. OSM is highly expressed in DCIS and IDC.

Figure 3.2: MDATO/OSM tumors increase metastasis and decrease survival.

Figure 3.3. Peri-tumoral OSM injections into mice with MDA-MB-231 D3H2LN tumors promote the development of metastases and CTCs.....126

Figure 3.4. Reduced OSM expression results in fewer spontaneous lung metastases and lower total volume of lung metastases by MRI. 128

Figure 3.5. Reduced tumor cell OSM expression increases survival in a 4T1.2-shOSM mouse model of tumor resection.

Figure 3.6. OSM promotes $4 \mathrm{~T} 1.2$ cell detachment and migration.

Figure 3.7. Model of OSM-mediated metastasis.

CHAPTER THREE: SUPPLEMENTAL FIGURES.

Figure 3.S1. Deterioration of body condition in MDATO/OSM tumor bearing mice treated with TET .136

Figure 3.S2. OSM is highly expressed in orthotopic 4T1.2 primary mammary tumors in female Balb/c mice .138

Figure 3.S3. qPCR analysis of lung metastases after intracardiac injections.

Supplemental Materials and Methods

Complete details for tissue microarrays.

Animal Histology

References

CHAPTER FOUR: HIGH EXPRESSION OF OSM AND IL-6 IS ASSOCIATED WITH DECREASED BREAST CANCER SURVIVAL: SYNERGISTIC INDUCTION OF IL-6 SECRETION BY OSM AND IL-1 $\beta$. .148

Authors .148 
Abstract

Introduction

Materials and Methods

Oncomine analysis

Enzyme linked immunosorbent assay.......

Cell lines

Stable and transient transfections.

Animal tumor xenograft model.

Immunoblot assay

Immunoprecipitation

Statistical analysis

Results

OSM and IL-6 expression are correlated with each other and associated with decreased invasive breast cancer survival

High OSM serum levels in breast cancer patients are correlated high IL-6 levels .....

OSM promotes IL-6 expression in a mouse model of human breast cancer.

OSM induces human IL-6 secretion in the absence of ER from various cancer cells in vitro

STAT3 is phosphorylated by OSM signaling in both ER- and ER+ cells

Invasive breast cancer patient stroma expresses high levels of OSM and $\mathrm{IL}-1 \beta$

OSM and IL-1 $\beta$ promote increased lymph node metastases and decreased survival: a three-way correlation for OSM-IL-1 $\beta$-IL-6 expression 164

OSM works synergistically with IL-1 $\beta$ to promote IL-6 secretion 165 
OSM and IL-1 $\beta$ activate separate signaling pathways to promote IL-6 production

ER interaction with STAT3 is suppressed by OSM and IL-1 $\beta$ in MCF7 cells

Discussion 168

Conclusions 172

Declarations 173

Chapter Four: Figures

Figure 4.1. OSM and IL-6 are associated with decreased invasive breast cancer survival

Figure 4. 2. OSM breast cancer patient serum levels correlate with IL-6 levels. 176

Figure 4.3. OSM induces IL-6 in an animal model of human breast cancer.

Figure 4.4. OSM induces IL-6 secretion in an ER-dependent manner ....181

Figure 4.5. OSM signals through STAT3 in ER- MDA-MB-231 cells to induce IL-6

Figure 4.6. OSM and IL-1 $\beta$ expression is higher in invasive breast cancer compared to normal tissue and correlates with higher lymph node metastasis, decreased survival, and IL-6 levels

Figure 4.7. OSM and IL-1 $\beta$ activate separate signaling pathways and synergistically induce IL-6 secretion

Figure 4.8. OSM and IL-1 $\beta$ promote IL-6 expression in a breast cancer cell-subtype specific manner

Chapter Four: Supplemental Figures

Figure 4.S1. OSM and IL-6 serum concentration is correlated in vivo...189

Figure 4.S2. IL-6 does not promote OSM secretion 190

Figure 4.S3. Stromal and tumor tissue expression of both OSM and IL-1 $\beta$ are higher in ER-patients 
Figure 4.S4. IL-1 $\beta$ mRNA expression levels correlate with both OSM and IL-6 in breast cancer patients ...............................................................193

Figure 4.S5. OSM and IL-1 $\beta$ promotes EMT-like morphology in T47D cells.

Figure 4.S6. ERK signaling is required for MCF7's synergistic response to OSM and IL-1 $\beta$

Figure 4.S7. STAT3 siRNA does not affect IL-1 $\beta$-induced IL-6 secretion.

Figure 4.S8. CAPE inhibits OSM-induced IL-6 secretion 198

References 


\section{LIST OF TABLES}

Table 3.1 Comparison of OSM expression in the ductal epithelial cells of different stages of ductal carcinoma of the breast (DCIS, IDC, and metastatic tissues) and adjacent normal breast tissues. 


\section{LIST OF FIGURES}

Figure 1.1 Chronic inflammation leads to genomic and epigenetic instability and promotes tumor formation.

Figure 1.2. Multi-step development of invasive ductal carcinoma (IDC).................. 24

Figure 1.3. Treatment strategies for breast cancer subtypes. …………………….... 25

Figure 1.4. Multiple signaling pathway activation in breast cancer cells. .................. 26

Figure 1.5. OSM induces the STAT3, PI3K, and MAPK pathways......................... 27

Figure 1.6. Tumor cells promote the development of angiogenesis. ......................... 28

Figure 2.1. Increased co-expression of OSM or OSMR $\beta$ and VEGF correlates with decreased survival of IDC patients ........................................................ 66

Figure 2.2. Co-expression of VEGF with OSM or OSMR $\beta$ appears to affect survival in HER2- but not HER2+ subtypes.......................................................... 68

Figure 2.3. IL-6 family cytokine OSM induces VEGF secretion independent of HIF $1 \alpha$ signaling in MDA-MB-231 cells .............................................................. 70

Figure 2.4. OSM strongly induces phosphorylation of STAT3 …............................ 71

Figure 2.5. OSM induces VEGF via STAT3 signaling............................................. 73

Figure 2.6. Mechanistically distinct regulation of VEGF secretion in MDA-MB-231 TNBC and T47D (ER+/PR+/HER2-) cells.............................................. 74

Figure 2.S1. Mechanistically distinct regulation of VEGF secretion in MDA-MB-231 TNBC and T47D (ER+/PR+/HER2-) cells........................................... 76

Figure 2.S2. Expression of cytokines or their receptors and VEGF appears to affect survival in HER2- but not HER2+ subtypes ............................................ 78

Figure 2.S3. Co-expression of OSM or OSMR $\beta$ and VEGF differentially affects survival based on estrogen receptor status 80

Figure 2.S4. OSM induces endothelial cell tube formation and branch points in vitro 82 
Figure 2.S5. OSM-treatment of human breast cancer cells stimulates angiogenesis in vivo 84

Figure 2.S6. JNK Inhibitor 2 suppresses VEGF secretion and phosphorylation of

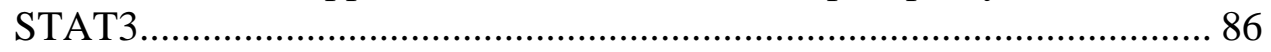

Figure 3.1. OSM is highly expressed in DCIS and IDC ...................................... 120

Figure 3.2. $\quad \mathrm{MDA}^{\mathrm{TO} / \mathrm{OSM}}$ tumors increase metastasis and decrease survival .............. 122

Figure 3.3. Peri-tumoral OSM injections into mice with MDA-MB-231-D3H2LN tumors promotes the development of metastases and CTCs .................. 125

Figure 3.4. Reduced OSM expression results in fewer spontaneous lung metastases and lower total volume of lung metastases by MRI ............................... 127

Figure 3.5. Reduced tumor cell OSM expression increases survival in a 4T1.2-shOSM mouse model of tumor resection........................................................ 129

Figure 3.6. OSM promotes 4T1.2 cell detachment and migration ............................. 131

Figure 3.7. Model of OSM-mediated metastasis...................................................... 133

Figure 3.S1. Deterioration of body condition in $\mathrm{MDA}^{\mathrm{TO} / \mathrm{OSM}}$ tumor bearing mice with TET 135

Figure 3.S2. OSM is highly expressed in orthotopic $4 \mathrm{~T} 1.2$ primary mammary tumors in female Balb/c mice 137

Figure 3.S3. qPCR analysis of lung metastases after intracardiac injections 139

Figure 4.1. OSM and IL-6 are associated with decreased invasive breast cancer survival. 174

Figure 4.2. OSM breast cancer patient serum levels correlate with IL-6 levels ....... 176

Figure 4.3. OSM induces IL-6 in an animal model of human breast cancer............. 178

Figure 4.4. OSM induces IL-6 secretion in an ER-dependent manner ..................... 180

Figure 4.5. OSM signals through STAT3 in ER- MDA-MB-231 cells to induce IL-6 182

Figure 4.6. OSM and IL-1 $\beta$ expression is higher in invasive breast cancer compared to normal tissue and correlates with higher lymph node metastasis, decreased survival, and IL-6 levels 184 
Figure 4.7. OSM and IL-1 $\beta$ activate separate signaling pathways and synergistically induce IL-6 secretion ..... 186

Figure 4.8. OSM and IL-1 $\beta$ promote IL-6 expression in a breast cancer cell-subtype specific manner

Figure 4.S1. OSM and IL-6 serum concentration is correlated in vivo ...................... 189

Figure 4.S2. IL-6 does not promote OSM secretion................................................... 190

Figure 4.S3. IL-6 does not promote OSM secretion.................................................. 191

Figure 4.S4. IL-1 $\beta$ mRNA expression levels correlate with both OSM and IL-6 in breast cancer patients ........................................................................... 193

Figure 4.S5. OSM and IL-1 $\beta$ promotes EMT-like morphology in T47D cells .......... 194

Figure 4.S6. ERK signaling is required for MCF7's synergistic response to OSM and

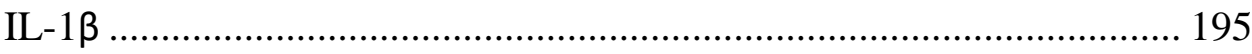

Figure 4.S7. STAT3 siRNA does not affect IL-1ß-induced IL-6 secretion ................. 197

Figure 4.S8. CAPE inhibits OSM-induced IL-6 secretion ........................................... 198 


\section{LIST OF ABBREVIATIONS}

$\begin{array}{ll}\text { CTC } & \text { Circulating tumor cells } \\ \text { DCIS } & \text { Ductal carcinoma in situ } \\ \text { ECM } & \text { Extracellular matrix } \\ \text { EMT } & \text { Epithelial to mesenchymal transition } \\ \text { ER } & \text { Estrogen receptor } \\ \text { FBS } & \text { Fetal bovine serum } \\ \text { FOV } & \text { Field of view } \\ \text { HER2 } & \text { Human epidermal growth factor receptor 2 } \\ \text { HIF1 } \alpha & \text { Hypoxia inducible factor 1 alpha } \\ \text { IDC } & \text { Invasive ductal carcinoma } \\ \text { IACUC } & \text { Institutional Animal Care and Use Committee } \\ \text { IL-1 } 3 & \text { Muclear factor kappa B } \\ \text { IL-6 } & \text { Interleukin-1 beta } \\ \text { IRB } & \text { Interleukin-6 } \\ \text { MAK } & \text { Institutional review board } \\ \text { MAPK } & \text { Matrin }\end{array}$


PI3K

PR

STAT3

TET

TE

TR

TMA

TME

TNBC

VEGF
Phosphoinositide 3-kinase

Progesterone receptor

Signal transducer and activator of transcription 3

Tetracycline

Exposure time

Repetition time

Tissue microarray

Tumor microenvironment

Triple negative breast cancer

Vascular endothelial growth factor 


\section{CHAPTER ONE: INTRODUCTION}

\section{A Brief Overview of Cancer}

Cancer describes a large group of diseases that commonly involve abnormal cell growth and invasion and the potential for metastasis to target organs. For the most part, it has been thought that cells become cancerous through a multi-step process that is typically due to DNA mutations. Mutations in genes that regulate cellular growth, cellcycle regulation genes, DNA repair genes, and a multitude of other genes related to cell transformation and migration are affected. However, by the turn of the century it became clear that changes in DNA sequences alone could not explain the extremely heterogeneous nature of malignancies seen by clinicians. Epigenetics, the process of shutting down or turning on genes through histone and DNA methylation or histone acetylation, gained a lot of attention by the end of the 1990's and was postulated as one of the main drivers of malignancy (1). Broadly speaking, epigenetic inhibition or stimulation of various genes that regulate the cell cycle and growth have been well documented. This role for epigenetics in cancer progression was a major blow to the Knudson's two-hit hypothesis, where mutations or damage to DNA were considered the main drivers for the transformation of normal cells into highly aggressive metastatic cancer cells. However, since methylation and acetylation can be targeted with various inhibitors, therapies that target oncogenic epigenetic events are possible (2). By 2010, a multitude of histone deacetylase (HDAC) inhibitors have been investigated for their 
potential role as therapeutics in certain malignancies (3). For example, HDAC inhibitors have been effective against hematological malignancies, but it is still unclear as to whether they will have positive clinical effect against solid tumors like breast cancer. Also, recent studies on ovarian cancer indicate that there may be some positive clinical potential for HDAC inhibitors in combination with chemotherapeutics (4).

While epigenetic aberrations are now known to be a major factor in the development of various malignancies, the etiological origins of these alterations in the development of cancer are not well defined. An interesting hypothesis has been developing over the past decade that postulates that inflammation can drive epigenetic alterations. In a study on cardiovascular disease, it was shown that inflammation promotes DNA hypermethylation and is a strong risk factor for the development of cardiovascular disease (5). Additional studies have shown a link between inflammation and epigenetics in a variety of other diseases ranging from macular degeneration, depression, and systemic sclerosis to cancer (6-9). It is also well known that localized inflammation and systematic inflammation have a strong dietary component and that pathological dietary habits have been shown to be a risk factor in many types of malignancies. Interestingly, diet, inflammation, and gut flora have been recently linked to epigenetic effects in colorectal cancer (10-12).

Inflammation is now a well-recognized driver of many aspects of malignancy from aberrant epigenetics to DNA damage, immune evasion, and cancer stem cell development (overview shown in Figure 1) $(13,14)$. Inflammation has become a potential target in cancer therapy through the use of specific inhibitors (14). This dissertation is a set of studies of specific inflammatory mediators and cytokines that 
promote the production of breast cancer growth factors, invasion, and metastasis, as well as decreased survival. Specifically, we show that proinflammatory factors such as oncostatin M (OSM) drive expression of other inflammatory cytokines, increase tumor angiogenesis, and the number of circulating tumor cells (CTCs) in breast cancer. Collectively, this body of research also provides a rationale for the development of antiOSM therapeutics for the treatment of metastatic breast cancer.

\section{Breast Cancer}

Global statistics indicate that breast cancer is the leading cause of cancer deaths among women worldwide (15). However, when just taking into consideration developed countries, breast cancer is the second leading cause of cancer deaths. This is due to the availability of better treatments, while the less treatable lung cancer remains the deadliest overall. In the United States, it is estimated that over 230,000 women will be diagnosed with breast cancer and over 40,000 breast cancer-related deaths are expected to occur in $2017(16,17)$. Of all of the types of breast cancer, invasive ductal carcinoma (IDC) is the most common and arises from the milk duct tissues in the breast (18). Breast cancer can also arise from other tissues in the breast, such as from the glandular lobes that results in lobular carcinoma, which make up $10-15 \%$ of all breast cancer, and from periductal stroma that results in phyllodes tumors, which are comparatively rare (19). Inflammatory breast cancer (IBC) is a very aggressive form of breast cancer, which accounts for less than $3 \%$ of all breast cancer cases (20). IBC typically start out as IDC where the cells shed and block lymphatic vessels, resulting in marked swelling and inflammation of the breast. 
During tumor progression, IDC starts as ductal hyperplasia, progresses to atypical hyperplasia with dysplasia, and then to ductal carcinoma in situ (DCIS), where the tumor cells start growing abnormally in the ductal tissue, but are not yet invasive (21) (Figure 2). Finally, when the tumor cells become malignant and acquire a more aggressive phenotype, they can penetrate through the basement membrane and into the local tumor microenvironment (TME). At this point, the tumor is designated invasive ductal carcinoma (IDC) and can potentially metastasize to distant organs through the lymphatic or the circulatory systems.

Significant morbidity and mortality associated with breast cancer comes from its metastasis to vital organs. The primary tumor itself is not typically lethal, as the breast is not considered essential for survival. Breast cancer most commonly metastasizes to lung, brain, liver, spine, and bone, all of which can lead to functional and structural disruptions in the target organs (22-25). Mechanisms that promote breast cancer metastasis are still not well understood, but it is thought that metastasis occurs by tumor cell dissemination through the lymphatic or the blood circulatory system. In most cases, cancer cells have to go through a phenotypic change called an epithelial-mesenchymal transition (EMT). This process causes the cells to change from an adherent, non-motile cell into a non-adherent, invasive, and highly mobile cell. Recent research indicates that the higher the tumor expression level of EMT markers, the more aggressive the breast cancer cells become and the worse the overall prognosis the patient experiences $(26,27)$.

\section{Breast Cancer Metastasis}

Once breast cancer cells become invasive they have the propensity to become metastatic and migrate to distant organs. For cancer to metastasize, it must undergo a 
multi-step process called the metastatic cascade, which involves the tumor cell's migration from the local TME through adjacent tissue into the circulatory system. For tumor cells to be able to move through local tissue barriers, the cells must first undergo an EMT $(28,29)$. In the process of EMT, epithelial cells that are not motile and maintain cell-cell and cell-matrix contact become mesenchymal, motile, and lose cell-to-cell contact. These mesenchymally-transitioned cells then enter the lymphatic or blood circulatory system in a process called intravasation $(29,30)$. Once the cells enter the circulation, they are designated as circulating tumor cells (CTCs) and can extravasate, or exit, into a target organ. CTCs that enter the circulation typically do not survive to form metastases, and in fact only $0.1 \%$ of all CTCs survive in the circulation (31), as most CTCs undergo apoptosis or are destroyed by the immune system. The few cells that do survive, extravasate into a target organ, undergo the reverse of EMT called a mesenchymal-epithelial transition (MET), and may either lay dormant (32) or develop into a secondary metastatic tumor (33).

Metastasis does not occur to just any organ but to sites where there is some level of microenvironment compatibility with the primary tumor cells such that the cells can implant and grow at the secondary site. Typically for breast cancer, some of the primary targets of metastases involve areas that are rich in capillaries and/or growth factors and include lung, brain, bone, and liver $(15,34-36)$. Once the cells have metastasized, target organ function is disrupted as the metastatic lesion grows and replaces healthy tissue. Bone metastases are particularly insidious as they cause marked morbidity and lead to rapid loss in quality of life, mobility loss, and intense pain that is refractory to opioid medication $(37,38)$. 
A common belief is that for cancer to metastasize, the tumor has to grow to a certain size before it is able to intravasate into the circulatory system. This is true for many types of cancer; however, recent studies show that for breast cancer, cells can metastasize as early as the DCIS stage when the cells are technically not supposed to be invasive $(39,40)$. This may necessitate a change in the clinical paradigm for the treatment of breast cancer, where new detection modalities need to be investigated. In addition, there may be other risk factors that affect early dissemination of breast cancer cells. For example, during systemic inflammation, inflammatory factors increase the propensity of the tumor cells to become invasive (41-43).

\section{Inflammation in Breast Cancer}

Many risky behavioral factors such as smoking, high alcohol consumption, sedentary lifestyle, depression, and high body mass index can promote chronic inflammation (44-46). Furthermore, poor dietary choices in adolescence with refined sugars and grains, processed meat, and low intake of vegetables have been linked to increased serum concentrations of inflammatory proteins such as C-reactive protein and interleukin-6 (IL-6) (46). Chronic breast inflammation arises from a multitude of factors including obesity, chronic infection, and poorly designed breast implants (47-49). Inflammation appears to drive breast cancer progression due to long-term insults associated with increased reactive oxygen species, DNA damage, mutation rate, and cell proliferation (49-54). Inflammatory cytokines such as IL-6 and others in the gp130 family are known for their role in both acute and chronic inflammation and in promoting cancer progression $(42,55-57)$. In particular, increased breast cancer recurrence after 
remission has been associated with poor health in connection to systemic inflammation (50).

Some of the downstream mediators of inflammation are cyclooxygenase-2 (COX2) and prostaglandin E2 (PGE2), which are both correlated with a poor breast cancer prognosis (58). Aromatase activity, which increases serum estrogen levels and promotes breast cancer cell growth, can be induced by PGE2 and other inflammatory mediators $(52,53,59)$. Interestingly, the use of non-steroidal anti-inflammatory drugs (NSAIDs) to suppress COX-2 has been associated with decreased risk of breast cancer (60).

Inflammation caused by various inflammatory mediators and cytokines in the gp130 family increase tumor angiogenesis, survival, immune evasion, and metrics associated with metastasis $(42,61,62)$. Interestingly, no matter what the etiological origin of IBC is, it is typically considered invariably fatal $(20,63)$. Together, these studies suggest that there is an intrinsic link between inflammation and breast cancer aggressiveness and possibly to breast cancer subtypes.

\section{Breast Cancer Subtypes}

Breast cancer is a highly heterogeneous disease where a multitude of genetic defects can lead to tumorigenesis and metastasis. Breast cancer aggressiveness roughly correlates with the tumor cells' expression of estrogen receptor (ER), progesterone receptor (PR), human epidermal growth factor receptor 2 (HER2), and Ki67, a marker for proliferation (64). Importantly, these markers are used as prognostic and therapeutic markers in the clinic. ER and PR are intracellular steroid receptors, and the cells expressing them tend to be dependent on estrogen and progesterone signaling for proliferation and survival $(65,66)$. When breast cancer cells lose their expression of ER 
and PR, they become estrogen- and progesterone-independent (also called ER- and PR-) and are unresponsive to therapies that ablate these hormones (67). When assessing ER and PR expression in tissue, a binary relationship exits at the cellular level, where each breast cancer cell either expresses or does not express ER and/or PR. On the other hand, HER2 expression is measured on a scale from low to high above the basal level of HER2 expression by normal breast epithelial cells. High HER2 expression (HER2 ${ }^{\mathrm{HIGH}}$ ), generally caused by gene amplification (68), constitutively activates various signaling pathways such as the PI3K/AKT and mitogen-activated protein kinase (MAPK) pathways and leads to the promotion of tumor cell proliferation, survival, and invasion (69) (65). Furthermore, this receptor can also gain a mutation where it becomes ligand-independent and is able to self-activate. When HER2 self-activates, the overall expression level of HER2 may still be low but the effects of HER2 are dramatically increased.

While ER and HER2 are therapeutically targetable markers, Ki-67 is primarily used as a marker for cell proliferation where high levels of Ki-67 staining $\left(\mathrm{Ki}^{6} 7^{\mathrm{HIGH}}\right)$ in the nucleus correlate to high levels of cellular mitosis (70). Unlike with ER, PR, and HER2 markers, Ki-67 is strictly used as a prognostic marker as it is not a very viable target for therapy due to the fact that nuclear proteins are very difficult to target with small molecule inhibitors. Currently there are no known therapies or clinical trials in progress for inhibition of $\mathrm{Ki}-67$.

Using these clinically relevant breast cancer markers, ER, PR, HER2, and Ki67, breast cancer can be subdivided into four subtypes. The breast cancer subtypes include Luminal A, Luminal B, Triple Negative/basal-like, and Her2-type (71) (Figure 3). Luminal A $\left(\mathrm{ER} / \mathrm{PR}+\mathrm{HER} 2^{\mathrm{LOW}} \mathrm{Ki} 67^{\mathrm{LOW}}\right)$ is the most frequent subtype in invasive 
cancers and is also the least aggressive with a good patient prognosis $(72,73)$. Luminal B $\left(\mathrm{ER} / \mathrm{PR}+, \mathrm{HER} 2^{\mathrm{HIGH}} \mathrm{Ki} 67^{\mathrm{HIGH}}\right)$ subtype is abundantly seen in ductal carcinoma in situ (DCIS) and is also not very aggressive. Patients with luminal B breast cancer also have a good prognosis. The most invasive breast cancer subtypes are triple negative breast cancer $(\mathrm{TNBC})\left(\mathrm{ER} / \mathrm{PR}-\mathrm{HER} 2^{\mathrm{LOW}} \mathrm{Ki} 67^{\mathrm{HIGH}}\right)$ and Her2-type (ER/PR- HER2 ${ }^{\mathrm{HIGH}}$ $\mathrm{Ki} 67^{\mathrm{HIGH}}$ ), which both have a poor overall prognosis (73). Contributing to the poor prognosis for patients with TNBC is the lack of targetability by therapeutic interventions, as the current anti-ER and anti-HER2 compounds are ineffective against cells lacking these targets.

\section{Breast Cancer Treatment}

Currently available breast cancer treatment modalities include the standard suite of chemotherapeutics and radiation treatment along with a handful of FDA-approved targeted therapies. ER and HER2 are the most targetable markers. When ER or HER2 levels are suppressed in the cancer cells that express them, a marked reduction in tumor cell growth results. Currently, ER expression is targeted by drugs such as tamoxifen and fulvestrant, while HER2 is inhibited by trastuzumab and lapatinib $(74,75)$. In addition, aromatase inhibitors such as letrozole are used to reduce the overall level of estrogen in the body to inhibit breast cancer cell growth. With these treatment modalities, both luminal A and B breast cancer can be effectively treated. As the disease progresses and becomes metastatic, it is more likely that the cancer will develop resistance to these therapies as they lose their therapeutically targetable markers and progress into TNBC. Since TNBC do not express any of these targetable markers, the existing drugs are ineffective in suppressing breast cancer progression. In essence, patients with TNBC 
have to rely on chemotherapy and radiation alone, and if they are fortunate, in combination with new, untested drugs in clinical trials (Figure 3) (76).

Other therapies such as gefitinib, erlotinib, and cetuximab, which all target the epithelial growth factor receptor (EGFR), have been used for TNBC patients in clinical trials (77). However, these therapies were not able to statistically improve patient outcomes when compared to standard treatment alone (77). Unlike with many other types of cancer, it is possible that TNBC is not dependent on EGFR signaling for tumor growth, survival, and invasive capacity. The lack of treatments for distant metastases makes metastatic TNBC the least survivable subtype with a 5-year survival rate of only $26 \%$ (17). This provides a rationale for the development of better anti-cancer therapeutics that are effective against metastatic breast cancer.

\section{Intracellular Pathways That Promote Breast Cancer Progression}

Inflammation-driven breast cancer is associated with various signaling pathways that are activated by inflammatory cytokines $(78,79)$. While there are a multitude of intracellular signaling pathways that affect gene regulation, only a handful are well known for promoting cancer progression. In breast cancer, pathways that are involved include the signal transducer and activator of transcription 3 (STAT3), phosphatidylinositol-3-kinase (PI3K), mitogen activated protein kinase (MAPK) pathways, and nuclear factor kappa B (NFאB) (79-85). Specifically, STAT3 signaling has been shown to increase breast cancer invasive potential by upregulating matrix metalloproteinases such as MMP2/9 (83) and has been associated with drug resistance to trastuzumab and doxorubicin $(80,82)$. The increase in MMPs by STAT3 signaling has also been shown to increase EMT, cancer cell detachment, and migration (86). 
Furthermore, PI3K is associated with increased AKT activation and promotes breast cancer cell survival against chemotherapeutics $(81,87)$. In addition, MAPK signaling promotes similar events as STAT3 and PI3K, including cell survival (69), drug resistance (88-90), and invasion (91), as well as promoting cell proliferation. NFאB signaling, on the other hand, has been shown to be associated with the development of breast cancer stem cells, which can repopulate metastases after tumor ablation by chemotherapeutics (92). Specifically, NFאB pathway activation been shown to increase breast cancer cell proliferation and invasion by increasing cluster of differentiation 44 (CD44) expression, which promotes cell migration, survival, and increased stem cell-like characteristics (85). Based on these studies it is quite evident that there is a lot of overlap in function between the STAT3, AKT, MAPK and NFkB signaling pathways in the context of breast cancer, suggesting that there could be synergistic effects when multiple pathways are activated (Figure 4). Indeed, other studies show that these pathways can be activated at the same time, which can promote a synergistic effect that drives complex breast cancer progression $(79,81)$. In summary, inflammation and inflammatory pathways related to the gp130 family of cytokines appear to be able to drive the simultaneous activation of these signaling pathways $(78,79)$.

\section{Oncostatin $M$ and Breast Cancer}

Oncostatin M (OSM), a gp130 family cytokine, acquired its name due to its cytostatic effect on breast cancer cells and was originally thought to be a possible therapeutic $(93,94)$. While this idea was popular between 1990-2000, it was later rebuked as new research demonstrated that OSM actually inhibits the expression of tumor suppressors $(95,96)$, induces EMT (97-100), recruits cancer-promoting neutrophils 
(101), and promotes metastasis (34). Furthermore, OSM signaling is associated with a poor breast cancer patient prognosis (97). OSM is secreted by a variety of cells in the tumor microenvironment such as tumor-associated neutrophils (TANs) and macrophages (TAMs), as well as adipocytes $(101,102)$. Interestingly, since OSM can be produced by adipose tissue, it expression levels may be increased in patients with obesity and type 2 diabetes, both of which are thought to be drivers in systemic inflammation-mediated breast cancer progression $(14,62,100,103)$. Furthermore, OSM appears to accumulate in the acidic breast cancer microenvironment, where it binds to ECM proteins and stays active $(98,104,105)$. Once OSM is secreted, it can act on target cells through the OSM receptor complex (OSMR), which consists of the gp130 protein dimerized with OSMR $\beta$ (106) (Figure 5). Once OSM binds to its receptor, it can activate a multitude of intracellular signaling cascades.

OSM is known to stimulate the activation of three signaling pathways including the STAT3 (107), PI3K/AKT (78), and MAPK pathways $(78,107)$ (Figure 5). Additionally, OSM rarely induces a single pathway in isolation, and multiple pathways become active at the same time (78). This leads to the multitude of OSM-induced cancerpromoting effects, which include increased vascular endothelial growth factor (VEGF) expression, EMT, tumor cell detachment, invasive capacity, and production of other proinflammatory factors such as $\mathrm{S} 100 \mathrm{~A} 7(34,78,97-99,108,109)$. Two other interesting effects associated with OSM are that it may push less aggressive breast cancer cells toward a more aggressive phenotype by suppressing ER expression and it may increase a cancer stem cell-like phenotype $(99,110,111)$. Therefore, it is thought that inflammation stimulated by cytokines such as OSM both promotes hormone-resistance as well as drives 
cancer cells towards a TNBC phenotype (111). Together, these studies strongly suggest that OSM is a proinflammatory factor with a multitude of effects that exacerbate breast cancer progression and metastasis. However, there are other related cytokines such as interleukin-1 beta (IL-1 $\beta$ ) and the parental gp130 family member IL-6, which can have synergistic and overlapping functions with OSM (112).

\section{Interleukin-6, And Interleukin-1 Beta in Breast Cancer}

IL-6 is a cytokine in the gp130 family and is known to bind to a receptor composed of a dimer of IL-6R $\alpha$ and a dimer of gp 130 to form a tetramer receptor complex (113). The entire signaling complex is composed of a hexameric assembly with two IL-6 cytokines, two IL-6R $\alpha$ subunits, and two gp130 subunits (113). IL-6 has been shown to activate multiple pathways including STAT3, JNK, and MAPK (114-116), which increase tumor cell growth, invasion, and metastasis $(42,114,117,118)$. These effects motivated the development of therapeutic agents to inhibit IL-6 signaling including chimeric or humanized antibodies against IL-6 and the IL-6R $\alpha(42,62,119)$. Anti-IL-6 therapeutic agents, such as tocilizumab that inhibits IL-6 and siltuximab that targets IL-6R $\alpha$, have been effective against Castleman's disease, which is characterized by high levels of IL-6 and abnormal proliferation of B and T immune cells $(120,121)$. However, despite siltuximab's effectiveness in limiting IL-6 signaling in patients, and despite IL-6's known effects in cancer progression, siltuximab has been ineffective in limiting the lethal effects of solid tumors $(122,123)$. This suggests that there may be additional inflammatory cytokines related to IL-6 that contribute to cancer invasion, metastasis, and mortality such as IL-1 $\beta$, which specifically induces IL-6 in breast cancer cells (124). 
IL-1 $\beta$ is a proinflammatory cytokine in the IL- 1 family, and it binds to a receptor complex composed of IL1-R1 and IL-1RAcP $(125,126)$. Once the receptor complex is activated, IL-1 $\beta$ induces the NFKB and MAPK pathways (127-129). IL-1 $\beta$ signaling is able to increase tumor cell growth, invasiveness, and metastasis in vivo by the stimulated secretion of proteases that degrade the ECM $(125,130-133)$. Anti-IL-1 $\beta$ therapeutics have been developed (134); however, much like with the anti-IL-6 biologics, IL-1 $\beta$ inhibition has not yielded positive results in patients when compared to standard chemotherapeutics (135). These studies suggest that for breast cancer patients, inhibition of a singular cytokine may not be enough due to the overlapping functions of these cytokines. Common anti-inflammatory medications such as NSAIDs inhibit cyclooxygenase 2 (COX2) expression, resulting in a decrease in prostaglandin production and in the reduced serum levels of a wide range of inflammatory cytokines and growth factors such as tumor necrosis factor alpha (TNF $\alpha$ ), IL-6, IL-1 $\beta$, and VEGF (136-138). Additionally, studies have shown that there may be a benefit in using NSAIDS for the reduction of breast cancer risk (60). In conclusion, multiple cytokines may need to be suppressed simultaneously to obtain a positive clinical effect with reduction in metastases and increased patient survival.

\section{Cancer Angiogenesis and Hypoxia Factors}

Angiogenesis is one of the hallmarks of cancer progression, as the development of new blood vessels is needed to oxygenate and feed the often highly metabolically active tumor. Angiogenesis starts when tumors reach $>1 \mathrm{~mm}$ in size, as this is the maximum distance for efficient diffusion of oxygen and nutrients from adjacent capillaries. Inflammation in the context of cancer has been associated with the production of the 
proangiogenic vascular endothelial growth factor (VEGF) (139-142) (Figure 6).

Furthermore, proangiogenic transcription factors such as hypoxia-inducible factor 1 (HIF1) have also been linked to inflammation, cancer stem cells, and cancer progression $(143,144)$. HIF 1 consists of a dimer of HIF1 $\alpha$ and HIF1 $\beta$. Typically, HIF $1 \alpha$ is regulated by rapid degradation while under normoxic conditions or in the absence of any inducing cytokines (145). Once HIF1 $\alpha$ has been stabilized by hypoxia or by HIF1-inducing cytokines, it can translocate to the nucleus after dimerizing with the constitutively expressed HIF1 $\beta$ and activate gene expression of VEGF and other proangiogenic proteins such as basic fibroblast growth factor (bFGF) $(146,147)$. In particular, OSM has been shown to increase HIF $1 \alpha$ levels through STAT3 activation that leads to an increase in the production of VEGF (148-150). IL-6 has also been shown to increase VEGF secretion through the induction of STAT3 phosphorylation (116). Despite the mountain of evidence showing that VEGF promotes breast cancer progression, anti-VEGF therapies have had limited success clinically (151). Much like with the story of the failed therapies with anti-IL-6 and anti-IL-1 $\beta$, it is likely that other proangiogenic factors play a role in promoting tumor angiogenesis. One of the consequences of tumor angiogenesis is that the tumor cells intravasate into the newly formed vessels and become circulating tumor cells.

\section{Circulating Tumor Cells and Their Detection}

Circulating tumor cells (CTCs) are an important element in cancer progression as they are thought to be the primary means by which tumors promote metastasis to distant organs (152). Specifically, high levels of CTCs in the blood of breast cancer patients have been correlated with poor prognosis and metastatic involvement in bone, lung, and other sites $(153,154)$. Current CTC detection methodologies involve the qPCR amplification 
of epithelial markers such as epithelial cell adhesion molecule (EpCAM), cytokeratin 19 (CK19), and human mammoglobin (hMAM) (155). Detection methods that are reliant on the presence of epithelial makers such as EpCAM and CK19 have run into a few major problems. For example, CTCs tend to lose their epithelial markers (such as EpCAM and CK19) when they undergo EMT and intravasate into the circulation $(156,157)$. Furthermore, detection methods using only epithelial markers have difficulty in determining if the CTC is a normal circulating epithelial cell or a tumor cell (158). Alternative methods include the use of a colony forming assay which exploits the immortalized nature of most tumor cells (159). With the colony forming assay, blood is collected from an individual, the red-blood cells are lysed with a hypotonic solution, and the resultant collection of cells, which include white blood cells and possible CTCs, are plated in tissue culture. Overtime, colonies of adherent tumor cells will grow, and each clonal colony is counted as one CTC. However, this method also has problems including the lengthy time involved in forming colonies and the fact that some tumor cell types fail to form colonies below a certain concentration of cells, which can lead to false negatives. Another alternative method for detecting CTCs includes the qPCR-based detection of human Alu repeat transposon sequences in human cancer cells. However, this method only works in in vivo xenograft mouse models where mouse cells have Alu repeat sequences that are different from the human tumor cell Alu repeats (160). Effective detection of CTCs remains problematic and controversial as the only FDA approved method for detection of CTCs uses the CELLSEARCH ${ }^{\circledR}$ technology, which depends on the detection of epithelial markers $(153,154,161)$. This necessitates further research into 
the nature of CTCs with a search for additional useful cancer cell markers that can be used in the detection of CTCs.

\section{Mouse Models for Breast Cancer}

The most common animal model for the study of tumorigenesis, metastasis, and drug development is the common mouse, mus musculus. The short life span and high metabolism of the mouse allows researchers to study the course of cancer progression in a compressed time-scale. Typical animal experiments using mus musculus for the study of cancer commonly last between 1-3 months, whereas in humans, studies take 1 to $5+$ years. Broadly speaking, there are three types of mouse cancer models: the transgenic tumor-forming mouse model, the human xenograft mouse model, and the syngeneic cancer mouse model (162-164). In the transgenic animal model, the animals are genetically modified to overexpress oncogenes such as c-Myc or have loss-of-function mutations in tumor suppressor genes such as p53 (164). These animals spontaneously develop tumors and are a useful model for studying neo-tumorigenesis where normal cells transform into metastatic tumor cells in a step-wise fashion.

On the other hand, xenograft and syngeneic mouse models require an injection of tumor cells into a target organ. Typically, cells from established breast cancer cell lines are injected orthotopically into the $4^{\text {th }}$ mammary fat pad of mice. With xenograft models, human breast cancer cells are used in immunodeficient mice such as in athymic nude mice, which do not have any functional T-cells (160). This prevents the immunodeficient animals from rejecting the human breast cancer cells and allows the study of human breast cancer in a living system. Alternatively, with a syngeneic mouse model, mouse mammary tumor cells derived from clonal mouse populations are injected into mice with 
the same genetic background. This allows the study of tumor growth and metastatic disease in a immunocompetent model (162). An example of such a model utilizes the 4T1 mammary tumor cell line that was derived from a spontaneous mammary tumor in a BALB/c albino mouse. The 4T1.2 cell line was developed by selecting a clonal population of $4 \mathrm{~T} 1$ cells and possesses a metastatic capacity that roughly matches what is seen in human breast cancer (165). An advantage of the syngeneic model is that the immune system is not compromised and inflammation and immunity, so important for metastasis, can be studied (41).

These animal models collectively give researchers powerful tools by which to study breast cancer progression, yet they are not without their faults. Many therapies that appear promising in in vivo animal models of breast cancer, fail miserably in the clinic. This underlies the need for better animal models that recapitulates human breast cancer (166). Although it is unlikely that a single animal model could ever perfectly model human breast cancer, utilization of multiple animal models may help confirm positive results. In addition, results from animal studies should be corroborated with available human data to increase the likelihood of the data being clinically relevant for human breast cancer.

\section{Human Data Analysis}

To assess the relevance of data derived from in vitro and in vivo experiments to human breast cancer, it is often important to corroborate this data with human patient samples and/or to data that is publicly available in various databases or repositories. An example of such a repository is Oncomine ${ }^{\mathrm{TM}}$, which includes over 18,000 cancer gene expression microarrays assessed from a wide variety of human cancers (167). Data can be 
downloaded from Oncomine ${ }^{\mathrm{TM}}$, and various types of gene expression and correlation analyses can be performed. Other sources of online databases include the catalog of somatic mutations in cancer (COSMIC), the human protein atlas, the cBioPortal cancer genomics database, and others (168-170). Combined with a few bioinformatics tools and some rudimentary PERL programming, these online databases can be a powerful source of data for researchers to associate in vitro or in vivo data with actual human cancer gene expression data.

Other sources of human patient data can be obtained from serum or tissues obtained from breast cancer patients. Serum and tissue samples can generally be acquired either from commercial sources and/or from hospitals/institutes that have a repository of stored human tissue and serum samples from patients. Alternatively, a new institutional review board protocol can be drafted to recruit patients for serum or tissue biopsy collection for cancer research, if the already available samples are insufficient or inappropriate for the study design. Additionally, serum samples can also be obtained from the National Cancer Institute's serum bank for various research studies (171). The collected samples can then be assessed by numerous assays ranging from immunohistochemistry, immunoblot analysis, and enzyme-linked immunosorbent assays (ELISAs), to laser capture microdissection and qPCR. Overall, these sources of samples and data provide researchers a diverse tool set and help move data from tissue culture and animal models closer to clinical relevance.

\section{Summary}

The work presented in the following studies primarily investigates the gp130 family of cytokines, including OSM and IL-6, in the context of cancer angiogenesis, 
metastasis, survival, and their synergistic relationship with other proinflammatory cytokines. In Chapter 2, co-expression of VEGF and gp130 family cytokines in relation to patient survival is assessed. These studies found that high expression levels of these proteins correlate with decreased breast cancer patient survival and that the level of VEGF production in response to OSM or IL-6 is breast cancer subtype-dependent. In Chapter 3, the role of OSM in the promotion of CTCs and lung metastases was investigated in multiple in vivo mouse models of breast cancer. Specifically, high levels of OSM, through injection of recombinant OSM or induction of OSM expression in mammary tumor-bearing mice, resulted in an increase in both CTC numbers and lung metastases, as well as in decreased survival. However, OSM had no effect on the survival of mice when the earlier steps of the metastatic cascade were bypassed by injecting tumor cells directly into circulation. This finding suggests that OSM is important early in the metastatic cascade. In Chapter 4, OSM induction of IL-6 was assessed in breast cancer. It was determined that OSM-induced IL-6 production is predicated on breast cancer cells lacking ER. Specifically, ER+ T47D and MCF7 did not produce any IL-6 in response to OSM, while ER+ TNBC MDA-MD-231 cells did. Furthermore, the synergistic relationship between OSM and IL1 $\beta$ was evaluated, and these studies demonstrate that OSM and IL1 $\beta$ activate separate signaling pathways to promote IL-6 production. Interestingly, increased breast tumor expression of OSM, IL-6, and IL1 $\beta$ were all correlated with reduced breast cancer patient survival, and all three cytokine expression patterns were correlated with each other. Overall, these studies attempt to elucidate the complex interplay of inflammatory cytokines in relation to breast cancer angiogenesis, 
metastasis, and survival and suggest that effective treatment of breast cancer may necessitate the suppression of multiple cytokines. 


\section{Figures}

A. Extrinsic pathway
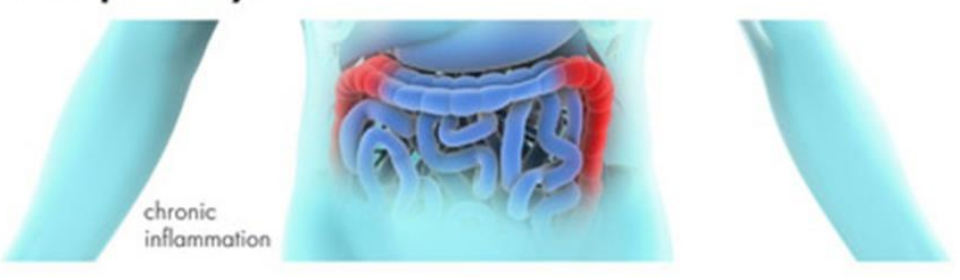

B. Genomic and epigenetic instability

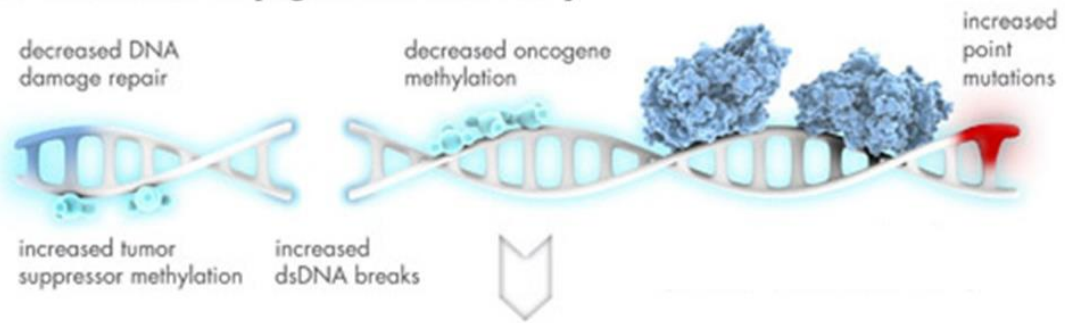

C. Inflammatory mediators
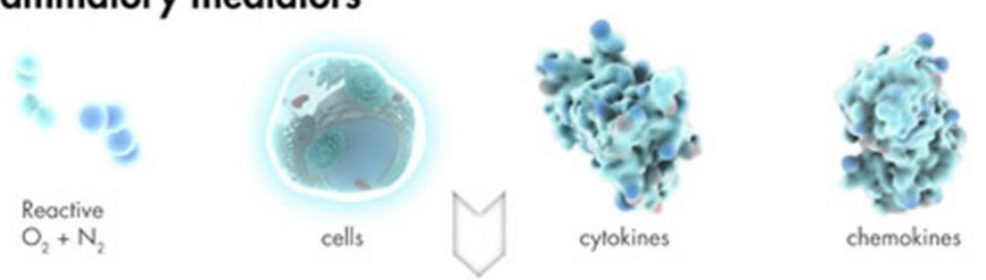

D. Transformation and metastasis

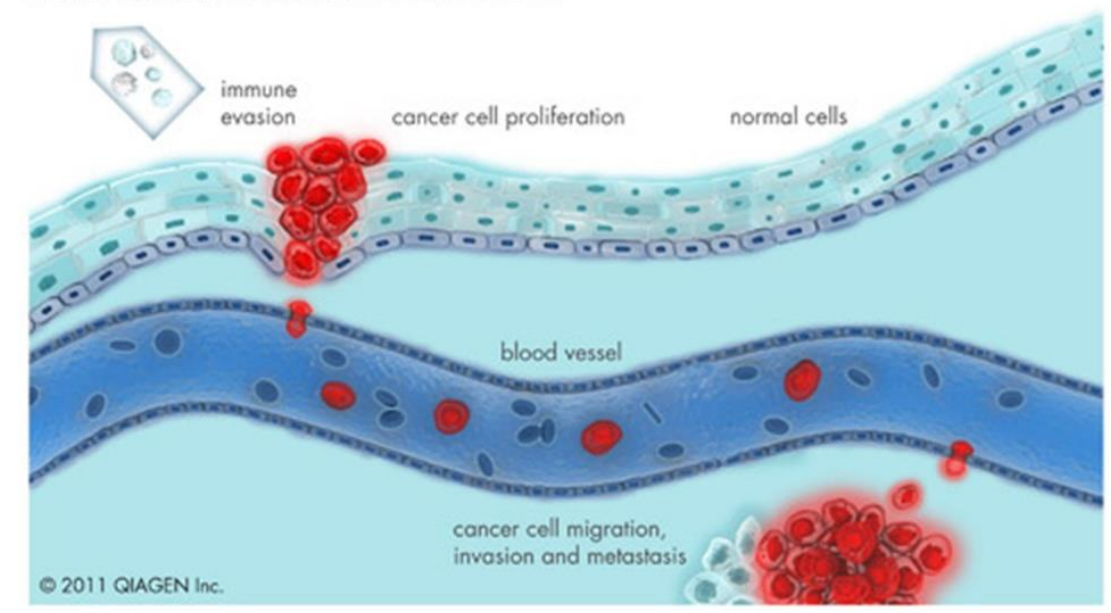

Figure 1.1 Chronic inflammation leads to genomic and epigenetic instability and promotes tumor formation. 
Figure 1.1 Chronic inflammation leads to genomic and epigenetic instability and promotes tumor formation

A, Chronic inflammation promotes the development of downstream procancerous effects seen in B. B, Inflammation increases genomic instability resulting from DNA damage and changes the epigenetic profile of various genes. $\mathbf{C}$, Genomic changes in precancerous cells can promote the secretion of inflammatory cytokines and chemokines in the tumor microenvironment and induce the development of reactive oxygen species. D, The cancer cells are then able to evade the immune system, increase cell proliferation, and invade into circulation. Once in circulation, the CTCs can then extravasate at distant organs and form a metastatic lesion. Adapted from QIAGEN $^{\odot}$ (172). 


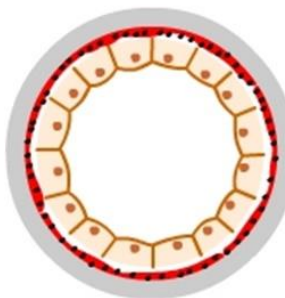

Normal duct

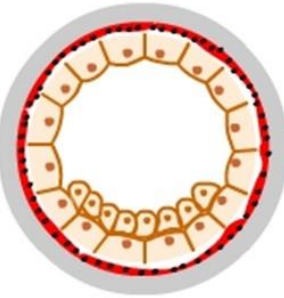

Ductal hyperplasia

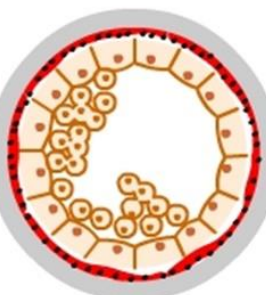

Atypical hyperplasia

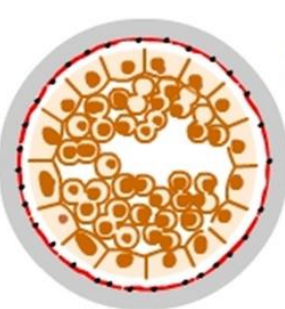

DCIS

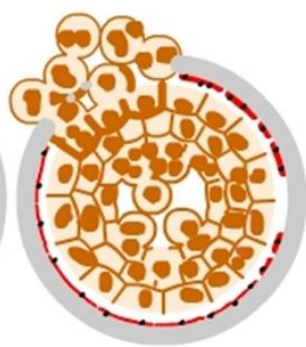

Invasive ductal carcinoma

Basement membrane

Myoepithelium

Ductal epithelium

(C) RnCeus.com

Figure 1.2. Multi-step development of invasive ductal carcinoma (IDC)

Figure 1.2. Multi-step development of invasive ductal carcinoma (IDC).

First, an epithelial cell lining the normal milk duct becomes hyperplastic and proliferates to induce a state called ductal hyperplasia. During atypical hyperplasia, cell organization is lost and cells lose their original shape (dysplasia). Next, it progresses into DCIS, where the normal ductal structure is lost, and a wide variety of cell sizes and nuclear morphologies present. Lastly, in IDC, the cells invade through the ductal basement membrane and may potentially metastasize to distant organs. Adapted from RnCeus.com ${ }^{\oplus}(173)$. 


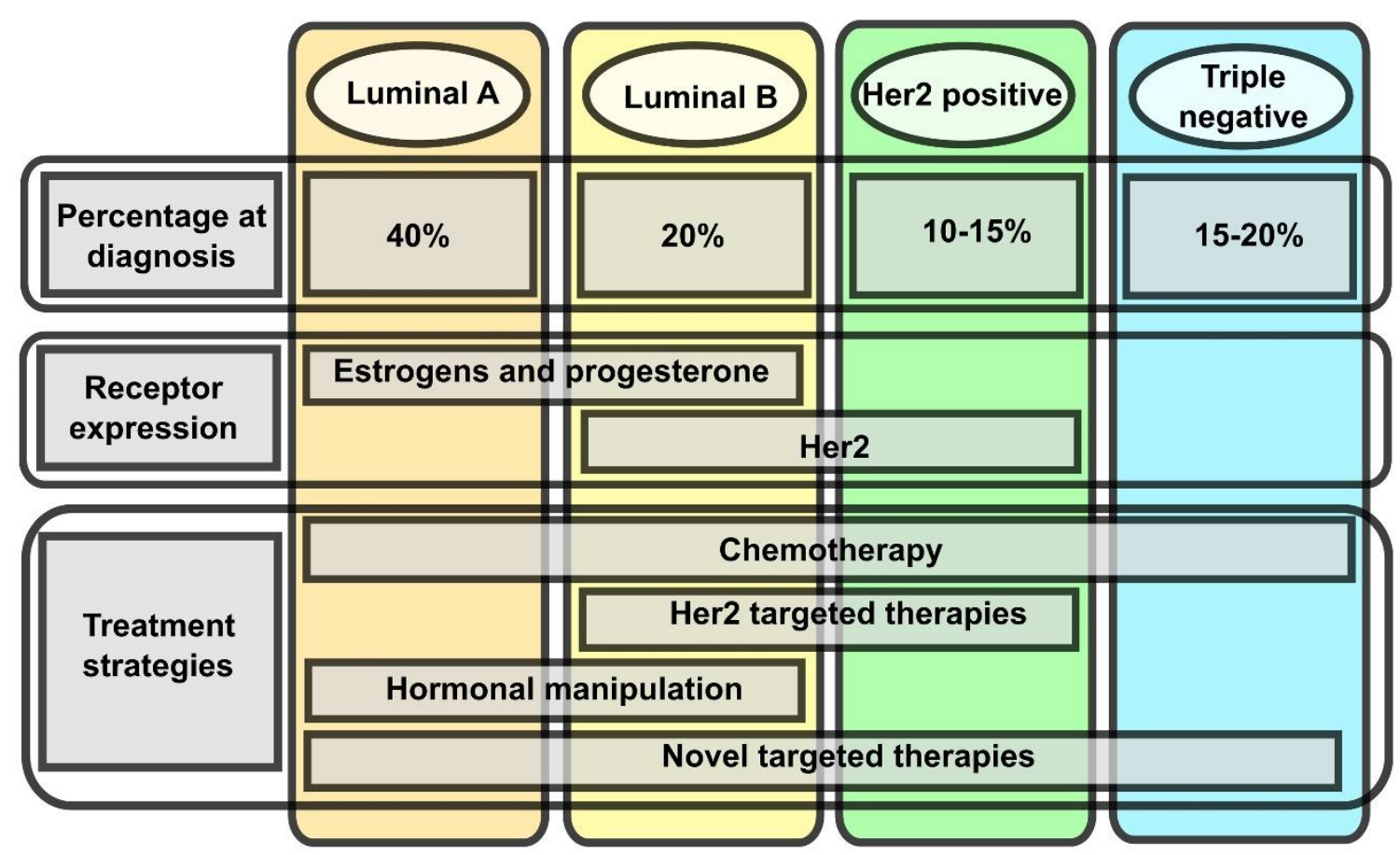

Figure 1.3. Treatment strategies for breast cancer subtypes.

Figure 1.3. Treatment strategies for breast cancer subtypes.

Luminal A and Luminal B are the most common types of breast cancer and are the most treatable; treatments include anti-hormone and/or anti-HER2 therapies along with traditional chemotherapy. HER2-positive, also known as HER2-type, breast cancer responds well to anti-HER2 therapeutics. TNBC on the other hand is the least treatable subtype of breast cancer, as it lacks the molecular targets that the current targeted therapies inhibit. TNBC patients must rely on chemotherapy and radiation alone and/or use novel therapies with unknown therapeutic potential. Adapted from Catalanotti V, et al. (76). 


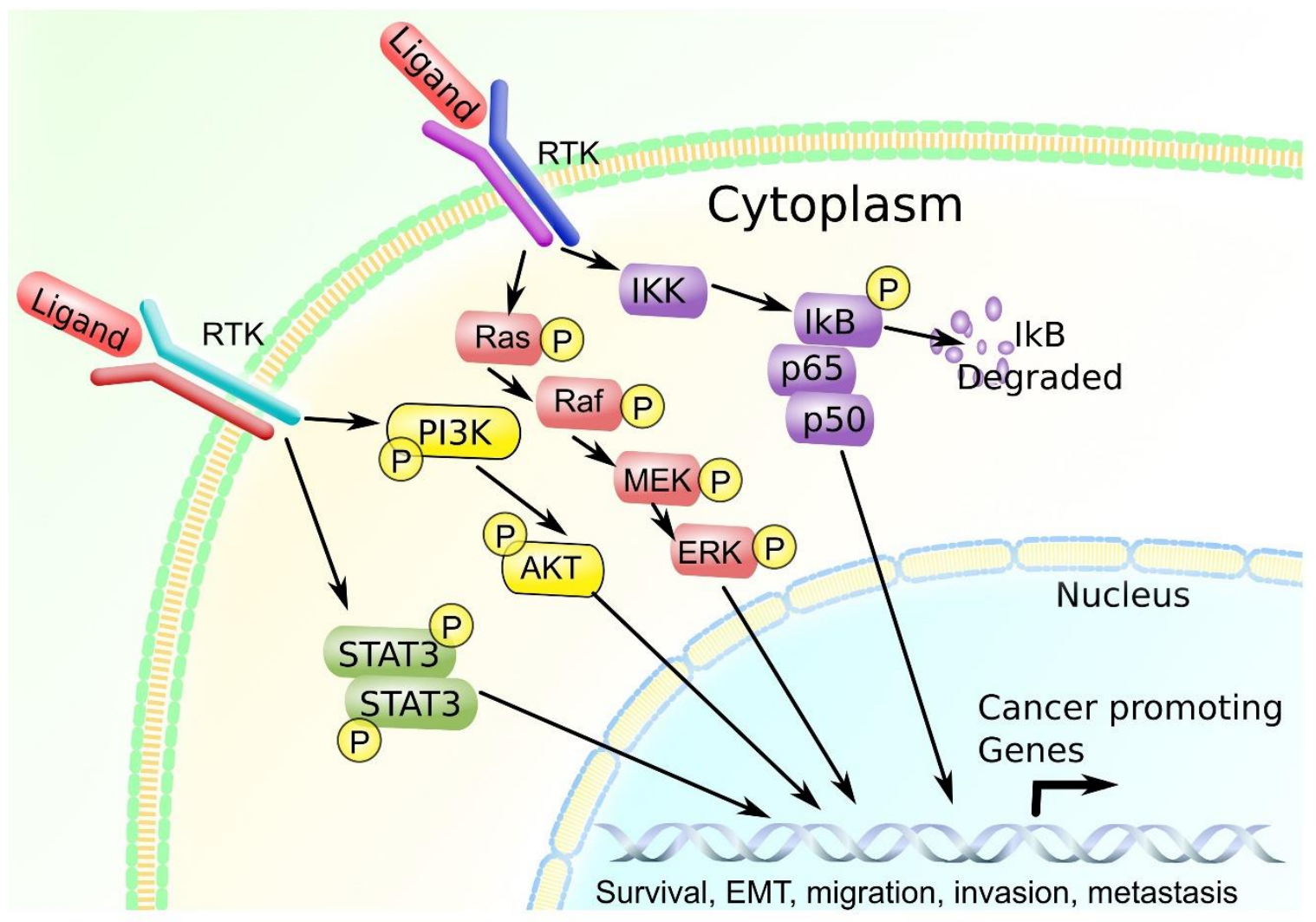

Figure 1.4. Multiple signaling pathway activation in breast cancer cells.

Figure 1.4. Multiple signaling pathway activation in breast cancer cells.

Some of the most common intracellular pathways that are active in breast cancer are the STAT3, PI3K/AKT, MAPK (MEK/ERK), and the NFkB (p65/p50) pathways. These pathways are activated in a variety of ways including the stimulation of receptor tyrosine kinases (RTK) by ligands such as growth factors and cytokines. Once these pathways are activated, usually through phosphorylation of target amino acids on the pathway proteins, they can lead to downstream effects such as tumor cell proliferation and survival, EMT, migration, invasion, and metastasis. 


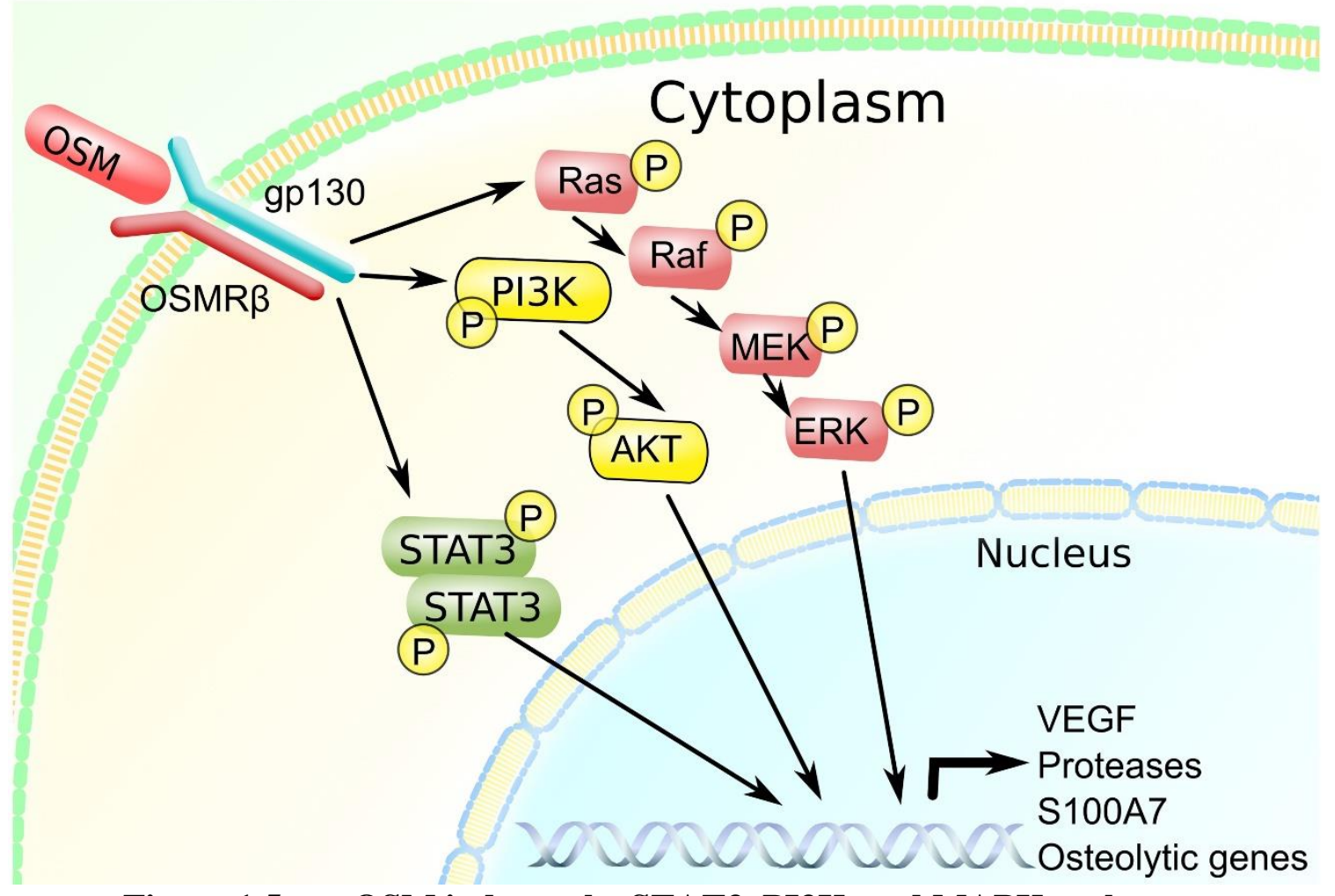

Figure 1.5. OSM induces the STAT3, PI3K, and MAPK pathways.

Figure 1.5. OSM induces the STAT3, PI3K, and MAPK pathways.

OSM is known to activate multiple signaling pathways including STAT3, AKT, and MAPK. Once these pathways are activated by phosphorylation of target signaling proteins, they can induce the translocation of transcription factors into the nucleus. Then transcription of downstream target genes such as VEGF, MMPs and other proteases, S100A7, and genes associated with osteolytic bone metastasis, all of which promote cancer progression. 


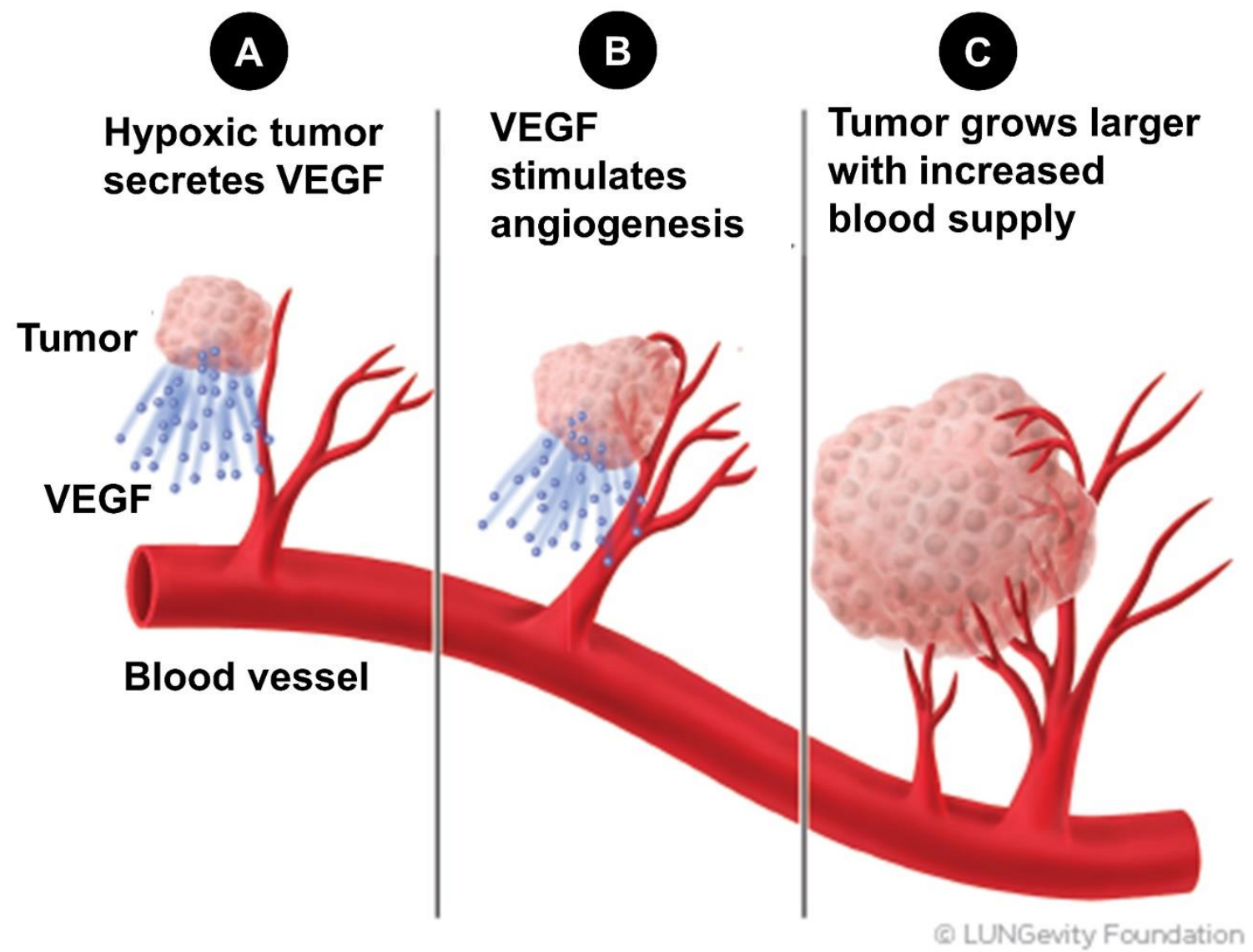

Figure 1.6. Tumor cells promote the development of angiogenesis.

Figure 1.6. Tumor cells promote the development of angiogenesis.

A, Once tumor cells reach a size greater than $1 \mathrm{~mm}$ in diameter, they may become hypoxic and release VEGF. B, VEGF stimulates angiogenesis whereby adjacent blood vessels proliferate, migrate, and form tubes sending new blood vessels into the tumor. This helps with tumor cell oxygenation, nutrient uptake, and overall growth. These new blood vessels tend to be leaky and it is thought that the porous nature of these immature blood vessels facilitate intravasation (174). C, As the tumor grows larger, the requirement for more blood supply is increased. Adapted from Lungevity ${ }^{\Theta}$ (175). 


\section{References}

1. Jones PA, Laird PW. Cancer epigenetics comes of age. Nat Genet. 1999;21(2):163-7. doi: 10.1038/5947. PubMed PMID: 9988266.

2. Yoo CB, Jones PA. Epigenetic therapy of cancer: past, present and future. Nat Rev Drug Discov. 2006;5(1):37-50. doi: 10.1038/nrd1930. PubMed PMID: 16485345.

3. Khan O, La Thangue NB. HDAC inhibitors in cancer biology: emerging mechanisms and clinical applications. Immunol Cell Biol. 2012;90(1):85-94. Epub 2011/11/29. doi: 10.1038/icb.2011.100. PubMed PMID: 22124371.

4. Akiyama M, Sowa Y, Taniguchi T, Watanabe M, Yogosawa S, Kitawaki J, Sakai T. Three-Combined Treatment, a Novel HDAC Inhibitor OBP-801/YM753, 5Fluorouracil and Paclitaxel, Induces G2-Phase Arrest Through the p38 Pathway in Human Ovarian Cancer Cells. Oncol Res. 2017. Epub 2017/01/23. doi: 10.3727/096504017X14850164661097. PubMed PMID: 28117030.

5. Stenvinkel P, Karimi M, Johansson S, Axelsson J, Suliman M, Lindholm B, Heimbürger O, Barany P, Alvestrand A, Nordfors L, Qureshi AR, Ekström TJ, Schalling M. Impact of inflammation on epigenetic DNA methylation - a novel risk factor for cardiovascular disease? J Intern Med. 2007;261(5):488-99. doi: 10.1111/j.1365-2796.2007.01777.x. PubMed PMID: 17444888.

6. Joyce BT, Gao T, Liu L, Zheng Y, Liu S, Zhang W, Penedo F, Dai Q, Schwartz J, Baccarelli AA, Hou L. Longitudinal Study of DNA Methylation of Inflammatory Genes and Cancer Risk. Cancer Epidemiol Biomarkers Prev. 2015;24(10):1531-8. Epub 2015/08/11. doi: 10.1158/1055-9965.EPI-15-0198. PubMed PMID: 26265203; PMCID: PMC4592468.

7. Gemenetzi M, Lotery AJ. The role of epigenetics in age-related macular degeneration. Eye (Lond). 2014;28(12):1407-17. Epub 2014/09/19. doi: 10.1038/eye.2014.225. PubMed PMID: 25233816; PMCID: PMC4268465.

8. Garfield L, Mathews HL, Witek Janusek L. Inflammatory and Epigenetic Pathways for Perinatal Depression. Biol Res Nurs. 2016;18(3):331-43. Epub 2015/11/16. doi: 10.1177/1099800415614892. PubMed PMID: 26574573.

9. Ciechomska M, van Laar JM, O'Reilly S. Emerging role of epigenetics in systemic sclerosis pathogenesis. Genes Immun. 2014;15(7):433-9. Epub 2014/07/17. doi: 10.1038/gene.2014.44. PubMed PMID: 25030429.

10. Han A, MacDonald A, Ahmed B, Whelan J, Donohoe D. Butyrate Regulates Its Own Metabolic Fate as an HDAC inhibitor in Colorectal Cancer Cells. The FASEB Journal. 2017;31(1 Supplement):300.2-.2.

11. Dashwood RH, Myzak MC, Ho E. Dietary HDAC inhibitors: time to rethink weak ligands in cancer chemoprevention? Carcinogenesis. 2006;27(2):344-9. Epub 2005/11/02. doi: 10.1093/carcin/bgi253. PubMed PMID: 16267097; PMCID: PMC2267878.

12. Lightfoot YL, Yang T, Sahay B, Mohamadzadeh M. Targeting aberrant colon cancer-specific DNA methylation with lipoteichoic acid-deficient Lactobacillus acidophilus. Gut Microbes. 2013;4(1):84-8. Epub 2012/11/08. doi: 10.4161/gmic.22822. PubMed PMID: 23137966; PMCID: PMC3555892. 
13. Wang S, Ma N, Zhao W, Midorikawa K, Kawanishi S, Hiraku Y, Oikawa S, Zhang Z, Huang G, Murata M. Inflammation-Related DNA Damage and Cancer Stem Cell Markers in Nasopharyngeal Carcinoma. Mediators Inflamm. 2016;2016:9343460. Epub 2016/08/28. doi: 10.1155/2016/9343460. PubMed PMID: 27647953; PMCID: PMC5018333.

14. Suarez-Carmona M, Lesage J, Cataldo D, Gilles C. EMT and inflammation: inseparable actors of cancer progression. Mol Oncol. 2017. Epub 2017/06/09. doi: 10.1002/1878-0261.12095. PubMed PMID: 28599100.

15. Torre LA, Bray F, Siegel RL, Ferlay J, Lortet-Tieulent J, Jemal A. Global cancer statistics, 2012. CA: a cancer journal for clinicians. 2015;65(2):87-108. doi: 10.3322/caac.21262. PubMed PMID: 25651787.

16. Society AC. Cancer Facts and Figures 2017. In: American Cancer Society I, editor. Atlanta2017. p. 1-56.

17. Society AC. Breast Cancer Facts and Figures 2015-2016. Atlanta: American Cancer Society, Inc; 2016.

18. Harris JR, Lippman ME, Veronesi U, Willett W. Breast cancer (2). N Engl J Med. 1992;327(6):390-8. doi: 10.1056/NEJM199208063270606. PubMed PMID: 1320737.

19. Tan BY, Acs G, Apple SK, Badve S, Bleiweiss IJ, Brogi E, Calvo JP, Dabbs DJ, Ellis IO, Eusebi V, Farshid G, Fox SB, Ichihara S, Lakhani SR, Rakha EA, Reis-Filho JS, Richardson AL, Sahin A, Schmitt FC, Schnitt SJ, Siziopikou KP, Soares FA, Tse GM, Vincent-Salomon A, Tan PH. Phyllodes tumours of the breast: a consensus review. Histopathology. 2016;68(1):5-21. doi: 10.1111/his.12876. PubMed PMID: 26768026; PMCID: PMC5027876.

20. Levine PH, Veneroso C. The epidemiology of inflammatory breast cancer. Semin Oncol. 2008;35(1):11-6. doi: 10.1053/j.seminoncol.2007.11.018. PubMed PMID: 18308141.

21. Bleicher RJ. Ductal carcinoma in situ. Surg Clin North Am. 2013;93(2):393-410. Epub 2013/01/29. doi: 10.1016/j.suc.2012.12.001. PubMed PMID: 23464692.

22. DeSantis CE, Fedewa SA, Goding Sauer A, Kramer JL, Smith RA, Jemal A. Breast cancer statistics, 2015: Convergence of incidence rates between black and white women. CA: a cancer journal for clinicians. 2015. doi: 10.3322/caac.21320. PubMed PMID: 26513636.

23. Trape AP, Gonzalez-Angulo AM. Breast cancer and metastasis: on the way toward individualized therapy. Cancer genomics \& proteomics. 2012;9(5):297-310. PubMed PMID: 22990109.

24. Diel IJ. Bone Metastasis in Breast Cancer. Breast care. 2012;7(2):90-1. doi: 10.1159/000338708. PubMed PMID: 22740793; PMCID: 3376358.

25. Scully OJ, Bay BH, Yip G, Yu Y. Breast cancer metastasis. Cancer genomics \& proteomics. 2012;9(5):311-20. PubMed PMID: 22990110.

26. Pomp V, Leo C, Mauracher A, Korol D, Guo W, Varga Z. Differential expression of epithelial-mesenchymal transition and stem cell markers in intrinsic subtypes of breast cancer. Breast cancer research and treatment. 2015. doi: 10.1007/s10549-015-3598-6. PubMed PMID: 26467042. 
27. Bae YK, Choi JE, Kang SH, Lee SJ. Epithelial-Mesenchymal Transition Phenotype Is Associated with Clinicopathological Factors That Indicate Aggressive Biological Behavior and Poor Clinical Outcomes in Invasive Breast Cancer. Journal of breast cancer. 2015;18(3):256-63. doi: 10.4048/jbc.2015.18.3.256. PubMed PMID: 26472976; PMCID: 4600690.

28. Liu H, Zhang X, Li J, Sun B, Qian H, Yin Z. The biological and clinical importance of epithelial-mesenchymal transition in circulating tumor cells. J Cancer Res Clin Oncol. 2015;141(2):189-201. Epub 2014/06/26. doi: 10.1007/s00432-014-1752-x. PubMed PMID: 24965746.

29. van Zijl F, Krupitza G, Mikulits W. Initial steps of metastasis: cell invasion and endothelial transmigration. Mutat Res. 2011;728(1-2):23-34. Epub 2011/05/12. doi: 10.1016/j.mrrev.2011.05.002. PubMed PMID: 21605699; PMCID: PMC4028085.

30. Carr I. Lymphatic metastasis. Cancer Metastasis Rev. 1983;2(3):307-17. PubMed PMID: 6367969.

31. Fidler IJ. Metastasis: quantitative analysis of distribution and fate of tumor emboli labeled with 125 I-5-iodo-2'-deoxyuridine. J Natl Cancer Inst. 1970;45(4):773-82. PubMed PMID: 5513503.

32. Price TT, Burness ML, Sivan A, Warner MJ, Cheng R, Lee CH, Olivere L, Comatas K, Magnani J, Kim Lyerly H, Cheng Q, McCall CM, Sipkins DA. Dormant breast cancer micrometastases reside in specific bone marrow niches that regulate their transit to and from bone. Sci Transl Med. 2016;8(340):340ra73. doi:

10.1126/scitranslmed.aad4059. PubMed PMID: 27225183.

33. Chaffer CL, Brennan JP, Slavin JL, Blick T, Thompson EW, Williams ED. Mesenchymal-to-epithelial transition facilitates bladder cancer metastasis: role of fibroblast growth factor receptor-2. Cancer Res. 2006;66(23):11271-8. doi:

10.1158/0008-5472.CAN-06-2044. PubMed PMID: 17145872.

34. Bolin C, Tawara K, Sutherland C, Redshaw J, Aranda P, Moselhy J, Anderson R, Jorcyk CL. Oncostatin m promotes mammary tumor metastasis to bone and osteolytic bone degradation. Genes \& cancer. 2012;3(2):117-30. doi: 10.1177/1947601912458284. PubMed PMID: 23050044; PMCID: 3463924.

35. Bos PD, Zhang XH, Nadal C, Shu W, Gomis RR, Nguyen DX, Minn AJ, van de Vijver MJ, Gerald WL, Foekens JA, Massagué J. Genes that mediate breast cancer metastasis to the brain. Nature. 2009;459(7249):1005-9. Epub 2009/05/06. doi: 10.1038/nature08021. PubMed PMID: 19421193; PMCID: PMC2698953.

36. Weigelt B, Peterse JL, van 't Veer LJ. Breast cancer metastasis: markers and models. Nat Rev Cancer. 2005;5(8):591-602. doi: 10.1038/nrc1670. PubMed PMID: 16056258 .

37. Petrut B, Trinkaus M, Simmons C, Clemons M. A primer of bone metastases management in breast cancer patients. Curr Oncol. 2008;15(Suppl 1):S50-7. PubMed PMID: 18231649; PMCID: PMC2216426.

38. Singh VA, Haseeb A, Alkubaisi AA. Incidence and outcome of bone metastatic disease at University Malaya Medical Centre. Singapore Med J. 2014;55(10):539-46. PubMed PMID: 25631896; PMCID: PMC4293965.

39. Husemann Y, Geigl JB, Schubert F, Musiani P, Meyer M, Burghart E, Forni G, Eils R, Fehm T, Riethmuller G, Klein CA. Systemic spread is an early step in 
breast cancer. Cancer Cell. 2008;13(1):58-68. doi: 10.1016/j.ccr.2007.12.003. PubMed PMID: 18167340.

40. Hosseini H, Obradović MM, Hoffmann M, Harper KL, Sosa MS, WernerKlein M, Nanduri LK, Werno C, Ehrl C, Maneck M, Patwary N, Haunschild G, Gužvić M, Reimelt C, Grauvogl M, Eichner N, Weber F, Hartkopf AD, Taran FA, Brucker SY, Fehm T, Rack B, Buchholz S, Spang R, Meister G, Aguirre-Ghiso JA, Klein CA. Early dissemination seeds metastasis in breast cancer. Nature. 2016. Epub 2016/12/14. doi: 10.1038/nature20785. PubMed PMID: 27974799; PMCID: PMC5390864.

41. Grivennikov SI, Greten FR, Karin M. Immunity, inflammation, and cancer. Cell. 2010;140(6):883-99. doi: 10.1016/j.cell.2010.01.025. PubMed PMID: 20303878; PMCID: PMC2866629.

42. Tawara K, Oxford JT, Jorcyk CL. Clinical significance of interleukin (IL)6 in cancer metastasis to bone: potential of anti-IL-6 therapies. Cancer management and research. 2011;3:177-89. doi: 10.2147/CMR.S18101. PubMed PMID: 21625400; PMCID: 3101113.

43. Crespi E, Bottai G, Santarpia L. Role of inflammation in obesity-related breast cancer. Curr Opin Pharmacol. 2016;31:114-22. Epub 2016/11/24. doi: 10.1016/j.coph.2016.11.004. PubMed PMID: 27889687.

44. Pierce BL, Neuhouser ML, Wener MH, Bernstein L, Baumgartner RN, Ballard-Barbash R, Gilliland FD, Baumgartner KB, Sorensen B, McTiernan A, Ulrich $\mathrm{CM}$. Correlates of circulating $\mathrm{C}$-reactive protein and serum amyloid A concentrations in breast cancer survivors. Breast Cancer Res Treat. 2009;114(1):155-67. Epub 2008/04/10. doi: 10.1007/s10549-008-9985-5. PubMed PMID: 18401703; PMCID: PMC3523176.

45. Ford DE, Erlinger TP. Depression and C-reactive protein in US adults: data from the Third National Health and Nutrition Examination Survey. Arch Intern Med. 2004;164(9):1010-4. doi: 10.1001/archinte.164.9.1010. PubMed PMID: 15136311.

46. Harris HR, Willett WC, Vaidya RL, Michels KB. An Adolescent and Early Adulthood Dietary Pattern Associated with Inflammation and the Incidence of Breast Cancer. Cancer Res. 2017;77(5):1179-87. doi: 10.1158/0008-5472.CAN-16-2273. PubMed PMID: 28249935; PMCID: PMC5335878.

47. Bhatelia K, Singh K, Singh R. TLRs: linking inflammation and breast cancer. Cellular signalling. 2014;26(11):2350-7. doi: 10.1016/j.cellsig.2014.07.035. PubMed PMID: 25093807.

48. Deng T, Lyon CJ, Bergin S, Caligiuri MA, Hsueh WA. Obesity, Inflammation, and Cancer. Annu Rev Pathol. 2016;11:421-49. doi: 10.1146/annurevpathol-012615-044359. PubMed PMID: 27193454.

49. Gubitosi A, Docimo G, Ruggiero R, Esposito A, Esposito E, Foroni F. Breast implant (PIP), chronic inflammation and cancer: is there a connection? Case report. Annali italiani di chirurgia. 2012;83. PubMed PMID: 23075481.

50. Cole SW. Chronic inflammation and breast cancer recurrence. Journal of clinical oncology : official journal of the American Society of Clinical Oncology. 2009;27(21):3418-9. doi: 10.1200/JCO.2009.21.9782. PubMed PMID: 19470918.

51. Hugo HJ, Saunders C, Ramsay RG, Thompson EW. New Insights on COX-2 in Chronic Inflammation Driving Breast Cancer Growth and Metastasis. Journal of mammary gland biology and neoplasia. 2015;20(3-4):109-19. doi: 10.1007/s10911015-9333-4. PubMed PMID: 26193871. 
52. Rose DP, Gracheck PJ, Vona-Davis L. The Interactions of Obesity, Inflammation and Insulin Resistance in Breast Cancer. Cancers. 2015;7(4):2147-68. doi: 10.3390/cancers7040883. PubMed PMID: 26516917.

53. Rose DP, Vona-Davis L. Biochemical and molecular mechanisms for the association between obesity, chronic inflammation, and breast cancer. BioFactors. 2014;40(1):1-12. doi: 10.1002/biof.1109. PubMed PMID: 23857944.

54. Suman S, Sharma PK, Rai G, Mishra S, Arora D, Gupta P, Shukla Y. Current perspectives of molecular pathways involved in chronic Inflammation-mediated breast cancer. Biochemical and biophysical research communications. 2015. doi: 10.1016/j.bbrc.2015.10.133. PubMed PMID: 26522220.

55. Brighenti E, Calabrese C, Liguori G, Giannone FA, Trere D, Montanaro L, Derenzini M. Interleukin 6 downregulates p53 expression and activity by stimulating ribosome biogenesis: a new pathway connecting inflammation to cancer. Oncogene. 2014;33(35):4396-406. doi: 10.1038/onc.2014.1. PubMed PMID: 24531714; PMCID: 4150990.

56. De Benedetti F, Alonzi T, Moretta A, Lazzaro D, Costa P, Poli V, Martini A, Ciliberto G, Fattori E. Interleukin 6 causes growth impairment in transgenic mice through a decrease in insulin-like growth factor-I. A model for stunted growth in children with chronic inflammation. The Journal of clinical investigation. 1997;99(4):643-50. doi: 10.1172/JCI119207. PubMed PMID: 9045866; PMCID: 507846.

57. Nguyen DP, Li J, Tewari AK. Inflammation and prostate cancer: the role of interleukin 6 (IL-6). BJU international. 2014;113(6):986-92. doi: 10.1111/bju.12452. PubMed PMID: 24053309.

58. Holmes MD, Chen WY, Schnitt SJ, Collins L, Colditz GA, Hankinson SE, Tamimi RM. COX-2 expression predicts worse breast cancer prognosis and does not modify the association with aspirin. Breast Cancer Res Treat. 2011;130(2):657-62. Epub 2011/07/05. doi: 10.1007/s10549-011-1651-7. PubMed PMID: 21728052; PMCID: PMC3292350.

59. Samarajeewa NU, Yang F, Docanto MM, Sakurai M, McNamara KM, Sasano H, Fox SB, Simpson ER, Brown KA. HIF-1 $\alpha$ stimulates aromatase expression driven by prostaglandin E2 in breast adipose stroma. Breast Cancer Res. 2013;15(2):R30. Epub 2013/04/08. doi: 10.1186/bcr3410. PubMed PMID: 23566437; PMCID: PMC3672802.

60. Takkouche B, Regueira-Méndez C, Etminan M. Breast cancer and use of nonsteroidal anti-inflammatory drugs: a meta-analysis. J Natl Cancer Inst. 2008;100(20):1439-47. Epub 2008/10/07. doi: 10.1093/jnci/djn324. PubMed PMID: 18840819.

61. Repovic P, Fears CY, Gladson CL, Benveniste EN. Oncostatin-M induction of vascular endothelial growth factor expression in astroglioma cells. Oncogene. 2003;22(50):8117-24. doi: 10.1038/sj.onc.1206922. PubMed PMID: 14603252.

62. Silver JS, Hunter CA. gp130 at the nexus of inflammation, autoimmunity, and cancer. J Leukoc Biol. 2010;88(6):1145-56. Epub 2010/07/07. doi: 10.1189/jlb.0410217. PubMed PMID: 20610800; PMCID: PMC2996896. 
63. Zhou J, Yan Y, Guo L, Ou H, Hai J, Zhang C, Wu Z, Tang L. Distinct outcomes in patients with different molecular subtypes of inflammatory breast cancer. Saudi Med J. 2014;35(11):1324-30. PubMed PMID: 25399208; PMCID: PMC4362150.

64. Kohler BA, Sherman RL, Howlader N, Jemal A, Ryerson AB, Henry KA, Boscoe FP, Cronin KA, Lake A, Noone AM, Henley SJ, Eheman CR, Anderson RN, Penberthy L. Annual Report to the Nation on the Status of Cancer, 1975-2011, Featuring Incidence of Breast Cancer Subtypes by Race/Ethnicity, Poverty, and State. J Natl Cancer Inst. 2015;107(6):djv048. Epub 2015/03/30. doi: 10.1093/jnci/djv048. PubMed PMID: 25825511; PMCID: PMC4603551.

65. Gangadhara S, Smith C, Barrett-Lee P, Hiscox S. 3D culture of Her2+ breast cancer cells promotes AKT to MAPK switching and a loss of therapeutic response. BMC Cancer. 2016;16:345. Epub 2016/06/01. doi: 10.1186/s 12885-016-2377-z. PubMed PMID: 27251376; PMCID: PMC4888214.

66. Jahandoost S, Farhanghian P, Abbasi S. The Effects of Sex Protein Receptors and Sex Steroid Hormone Gene Polymorphisms on Breast Cancer Risk. J Natl Med Assoc. 2017;109(2):126-38. Epub 2017/03/01. doi: 10.1016/j.jnma.2017.02.003. PubMed PMID: 28599754.

67. Gangadhara S, Barrett-Lee P, Nicholson RI, Hiscox S. Pro-metastatic tumor-stroma interactions in breast cancer. Future Oncol. 2012;8(11):1427-42. doi: 10.2217/fon.12.134. PubMed PMID: 23148616.

68. Dybdal N, Leiberman G, Anderson S, McCune B, Bajamonde A, Cohen RL, Mass RD, Sanders C, Press MF. Determination of HER2 gene amplification by fluorescence in situ hybridization and concordance with the clinical trials immunohistochemical assay in women with metastatic breast cancer evaluated for treatment with trastuzumab. Breast Cancer Res Treat. 2005;93(1):3-11. doi: 10.1007/s10549-004-6275-8. PubMed PMID: 16184453.

69. Menendez JA, Vellon L, Mehmi I, Teng PK, Griggs DW, Lupu R. A novel CYR61-triggered 'CYR61-alphavbeta3 integrin loop' regulates breast cancer cell survival and chemosensitivity through activation of ERK1/ERK2 MAPK signaling pathway. Oncogene. 2005;24(5):761-79. doi: 10.1038/sj.onc.1208238. PubMed PMID: 15592521.

70. Scholzen T, Gerdes J. The Ki-67 protein: from the known and the unknown. J Cell Physiol. 2000;182(3):311-22. doi: 10.1002/(SICI)10974652(200003)182:3<311::AID-JCP1>3.0.CO;2-9. PubMed PMID: 10653597.

71. Tamimi RM, Baer HJ, Marotti J, Galan M, Galaburda L, Fu Y, Deitz AC, Connolly JL, Schnitt SJ, Colditz GA, Collins LC. Comparison of molecular phenotypes of ductal carcinoma in situ and invasive breast cancer. Breast Cancer Res. 2008;10(4):R67. Epub 2008/08/05. doi: 10.1186/bcr2128. PubMed PMID: 18681955; PMCID: PMC2575540.

72. Sørlie T, Perou CM, Tibshirani R, Aas T, Geisler S, Johnsen H, Hastie T, Eisen MB, van de Rijn M, Jeffrey SS, Thorsen T, Quist H, Matese JC, Brown PO, Botstein D, Lønning PE, Børresen-Dale AL. Gene expression patterns of breast carcinomas distinguish tumor subclasses with clinical implications. Proc Natl Acad Sci U S A. 2001;98(19):10869-74. doi: 10.1073/pnas.191367098. PubMed PMID: 11553815; PMCID: PMC58566. 
73. Soliman NA, Yussif SM. Ki-67 as a prognostic marker according to breast cancer molecular subtype. Cancer Biol Med. 2016;13(4):496-504. doi: 10.20892/j.issn.2095-3941.2016.0066. PubMed PMID: 28154782; PMCID: PMC5250608.

74. Macedo LF, Sabnis G, Brodie A. Aromatase inhibitors and breast cancer. Ann N Y Acad Sci. 2009;1155:162-73. doi: 10.1111/j.1749-6632.2008.03689.x. PubMed PMID: 19250202.

75. Arkin M, Moasser MM. HER-2-directed, small-molecule antagonists. Curr Opin Investig Drugs. 2008;9(12):1264-76. PubMed PMID: 19037833; PMCID: PMC3031872.

76. Catalanotti V BV, Tariq N, Califano R. Treatment of Advanced Breast Cancer (ABC): The Expanding Landscape of Targeted Therapies. J Cancer Biol Res. 2014;2(1):1036

77. Nakai K, Hung MC, Yamaguchi H. A perspective on anti-EGFR therapies targeting triple-negative breast cancer. Am J Cancer Res. 2016;6(8):1609-23. Epub 2016/08/01. PubMed PMID: 27648353; PMCID: PMC5004067.

78. Demyanets S, Kaun C, Rychli K, Pfaffenberger S, Kastl SP, Hohensinner PJ, Rega G, Katsaros KM, Afonyushkin T, Bochkov VN, Paireder M, Huk I, Maurer G, Huber K, Wojta J. Oncostatin M-enhanced vascular endothelial growth factor expression in human vascular smooth muscle cells involves PI3K-, p38 MAPK-, Erk1/2- and STAT1/STAT3-dependent pathways and is attenuated by interferon-gamma. Basic research in cardiology. 2011;106(2):217-31. doi: 10.1007/s00395-010-0141-0. PubMed PMID: 21174212.

79. Kim MS, Lee WS, Jeong J, Kim SJ, Jin W. Induction of metastatic potential by TrkB via activation of IL6/JAK2/STAT3 and PI3K/AKT signaling in breast cancer. Oncotarget. 2015;6(37):40158-71. doi: 10.18632/oncotarget.5522. PubMed PMID: 26515594.

80. Gariboldi MB, Ravizza R, Molteni R, Osella D, Gabano E, Monti E. Inhibition of Stat 3 increases doxorubicin sensitivity in a human metastatic breast cancer cell line. Cancer letters. 2007;258(2):181-8. doi: 10.1016/j.canlet.2007.08.019. PubMed PMID: 17920763.

81. Kim JW, Kim DK, Min A, Lee KH, Nam HJ, Kim JH, Kim JS, Kim TY, Im SA, Park IA. Amphiregulin confers trastuzumab resistance via AKT and ERK activation in HER2-positive breast cancer. Journal of cancer research and clinical oncology. 2015. doi: 10.1007/s00432-015-2012-4. PubMed PMID: 26195282.

82. Sonnenblick A, Brohee S, Fumagalli D, Vincent D, Venet D, Ignatiadis M, Salgado R, Van den Eynden G, Rothe F, Desmedt C, Neven P, Loibl S, Denkert C, Joensuu H, Loi S, Sirtaine N, Kellokumpu-Lehtinen PL, Piccart M, Sotiriou C. Constitutive phosphorylated STAT3-associated gene signature is predictive for trastuzumab resistance in primary HER2-positive breast cancer. BMC medicine. 2015;13:177. doi: 10.1186/s12916-015-0416-2. PubMed PMID: 26234940; PMCID: 4522972.

83. Zhang F, Wang Z, Fan Y, Xu Q, Ji W, Tian R, Niu R. Elevated STAT3 Signaling-Mediated Upregulation of MMP-2/9 Confers Enhanced Invasion Ability in Multidrug-Resistant Breast Cancer Cells. International journal of molecular sciences. 
2015;16(10):24772-90. doi: 10.3390/ijms161024772. PubMed PMID: 26501276; PMCID: 4632776.

84. Zhang H, Cai K, Wang J, Wang X, Cheng K, Shi F, Jiang L, Zhang Y, Dou J. MiR-7, inhibited indirectly by lincRNA HOTAIR, directly inhibits SETDB1 and reverses the EMT of breast cancer stem cells by downregulating the STAT3 pathway. Stem cells. 2014;32(11):2858-68. doi: 10.1002/stem.1795. PubMed PMID: 25070049.

85. Smith SM, Lyu YL, Cai L. NF- $\mathrm{kB}$ affects proliferation and invasiveness of breast cancer cells by regulating CD44 expression. PLoS One. 2014;9(9):e106966. Epub 2014/09/03. doi: 10.1371/journal.pone.0106966. PubMed PMID: 25184276; PMCID: PMC4153718.

86. Tester AM, Ruangpanit N, Anderson RL, Thompson EW. MMP-9 secretion and MMP-2 activation distinguish invasive and metastatic sublines of a mouse mammary carcinoma system showing epithelial-mesenchymal transition traits. Clin Exp Metastasis. 2000;18(7):553-60. PubMed PMID: 11688960.

87. Chen X, Wang H, Ou-Yang XN, Xie FW, Wu JJ. Research on drug resistance mechanism of trastuzumab caused by activation of the PI3K/Akt signaling pathway. Contemporary oncology. 2013;17(4):363-9. doi: 10.5114/wo.2013.35292. PubMed PMID: 24592124; PMCID: 3934046.

88. Donovan JC, Milic A, Slingerland JM. Constitutive MEK/MAPK activation leads to $\mathrm{p} 27$ (Kip1) deregulation and antiestrogen resistance in human breast cancer cells. The Journal of biological chemistry. 2001;276(44):40888-95. doi: 10.1074/jbc.M106448200. PubMed PMID: 11527971.

89. Eralp Y, Derin D, Ozluk Y, Yavuz E, Guney N, Saip P, Muslumanoglu M, Igci A, Kucucuk S, Dincer M, Aydiner A, Topuz E. MAPK overexpression is associated with anthracycline resistance and increased risk for recurrence in patients with triplenegative breast cancer. Annals of oncology : official journal of the European Society for Medical Oncology / ESMO. 2008;19(4):669-74. doi: 10.1093/annonc/mdm522. PubMed PMID: 18006896.

90. Normanno N, Campiglio M, Maiello MR, De Luca A, Mancino M, Gallo M, D'Alessio A, Menard S. Breast cancer cells with acquired resistance to the EGFR tyrosine kinase inhibitor gefitinib show persistent activation of MAPK signaling. Breast cancer research and treatment. 2008;112(1):25-33. doi: 10.1007/s10549-007-9830-2. PubMed PMID: 18060492.

91. Meng X, Cai C, Wu J, Cai S, Ye C, Chen H, Yang Z, Zeng H, Shen Q, Zou F. TRPM7 mediates breast cancer cell migration and invasion through the MAPK pathway. Cancer letters. 2013;333(1):96-102. doi: 10.1016/j.canlet.2013.01.031. PubMed PMID: 23353055.

92. Shostak K, Chariot A. NF-KB, stem cells and breast cancer: the links get stronger. Breast Cancer Res. 2011;13(4):214. Epub 2011/07/26. doi: 10.1186/bcr2886. PubMed PMID: 21867572; PMCID: PMC3236328.

93. Liu J, Spence MJ, Wallace PM, Forcier K, Hellstrom I, Vestal RE. Oncostatin M-specific receptor mediates inhibition of breast cancer cell growth and down-regulation of the c-myc proto-oncogene. Cell growth \& differentiation : the molecular biology journal of the American Association for Cancer Research. 1997;8(6):667-76. PubMed PMID: 9186000. 
94. Spence MJ, Vestal RE, Liu J. Oncostatin M-mediated transcriptional suppression of the c-myc gene in breast cancer cells. Cancer research. 1997;57(11):22238. PubMed PMID: 9187125.

95. Li C, Ahlborn TE, Tokita K, Boxer LM, Noda A, Liu J. The critical role of the PE21 element in oncostatin M-mediated transcriptional repression of the p53 tumor suppressor gene in breast cancer cells. Oncogene. 2001;20(57):8193-202. doi: 10.1038/sj.onc.1205046. PubMed PMID: 11781835.

96. Liu J, Li C, Ahlborn TE, Spence MJ, Meng L, Boxer LM. The expression of p53 tumor suppressor gene in breast cancer cells is down-regulated by cytokine oncostatin M. Cell growth \& differentiation : the molecular biology journal of the American Association for Cancer Research. 1999;10(10):677-83. PubMed PMID: 10547071.

97. West NR, Murphy LC, Watson PH. Oncostatin M suppresses oestrogen receptor-alpha expression and is associated with poor outcome in human breast cancer. Endocrine-related cancer. 2012;19(2):181-95. doi: 10.1530/ERC-11-0326. PubMed PMID: 22267707.

98. Ryan RE, Martin B, Mellor L, Jacob RB, Tawara K, McDougal OM, Oxford JT, Jorcyk CL. Oncostatin M binds to extracellular matrix in a bioactive conformation: implications for inflammation and metastasis. Cytokine. 2015;72(1):71-85. doi: 10.1016/j.cyto.2014.11.007. PubMed PMID: 25622278; PMCID: 4328881.

99. West NR, Murray JI, Watson PH. Oncostatin-M promotes phenotypic changes associated with mesenchymal and stem cell-like differentiation in breast cancer. Oncogene. 2014;33(12):1485-94. doi: 10.1038/onc.2013.105. PubMed PMID: 23584474.

100. Lapeire L, Hendrix A, Lambein K, Van Bockstal M, Braems G, Van Den Broecke R, Limame R, Mestdagh P, Vandesompele J, Vanhove C, Maynard D, Lehuédé C, Muller C, Valet P, Gespach CP, Bracke M, Cocquyt V, Denys H, De Wever O. Cancer-associated adipose tissue promotes breast cancer progression by paracrine oncostatin M and Jak/STAT3 signaling. Cancer Res. 2014;74(23):6806-19. Epub 2014/09/24. doi: 10.1158/0008-5472.CAN-14-0160. PubMed PMID: 25252914.

101. Queen MM, Ryan RE, Holzer RG, Keller-Peck CR, Jorcyk CL. Breast cancer cells stimulate neutrophils to produce oncostatin $\mathrm{M}$ : potential implications for tumor progression. Cancer research. 2005;65(19):8896-904. doi: 10.1158/00085472.CAN-05-1734. PubMed PMID: 16204061.

102. Guihard P, Boutet MA, Brounais-Le Royer B, Gamblin AL, Amiaud J, Renaud A, Berreur M, Rédini F, Heymann D, Layrolle P, Blanchard F. Oncostatin m, an inflammatory cytokine produced by macrophages, supports intramembranous bone healing in a mouse model of tibia injury. Am J Pathol. 2015;185(3):765-75. Epub 2015/01/02. doi: 10.1016/j.ajpath.2014.11.008. PubMed PMID: 25559270.

103. Sanchez-Infantes D, White UA, Elks CM, Morrison RF, Gimble JM, Considine RV, Ferrante AW, Ravussin E, Stephens JM. Oncostatin $m$ is produced in adipose tissue and is regulated in conditions of obesity and type 2 diabetes. J Clin Endocrinol Metab. 2014;99(2):E217-25. Epub 2013/12/02. doi: 10.1210/jc.2013-3555. PubMed PMID: 24297795; PMCID: PMC3913819.

104. Kato Y, Ozawa S, Miyamoto C, Maehata Y, Suzuki A, Maeda T, Baba Y. Acidic extracellular microenvironment and cancer. Cancer Cell Int. 2013;13(1):89. Epub 
2013/09/03. doi: 10.1186/1475-2867-13-89. PubMed PMID: 24004445; PMCID: PMC3849184.

105. Yoneda T, Hiasa M, Nagata Y, Okui T, White F. Contribution of acidic extracellular microenvironment of cancer-colonized bone to bone pain. Biochim Biophys Acta. 2015;1848(10 Pt B):2677-84. Epub 2015/02/14. doi: 10.1016/j.bbamem.2015.02.004. PubMed PMID: 25687976; PMCID: PMC5356024.

106. Le Goff B, Singbrant S, Tonkin BA, Martin TJ, Romas E, Sims NA, Walsh NC. Oncostatin M acting via OSMR, augments the actions of IL-1 and TNF in synovial fibroblasts. Cytokine. 2014;68(2):101-9. Epub 2014/04/22. doi: 10.1016/j.cyto.2014.04.001. PubMed PMID: 24767864.

107. Kurosawa T, Yamada A, Takami M, Suzuki D, Saito Y, Hiranuma K, Enomoto T, Morimura N, Yamamoto M, Iijima T, Shirota T, Itabe H, Kamijo R. Expression of nephronectin is inhibited by oncostatin M via both JAK/STAT and MAPK pathways. FEBS open bio. 2015;5:303-7. doi: 10.1016/j.fob.2015.04.001. PubMed PMID: 25905035; PMCID: 4404411.

108. West NR, Watson PH. S100A7 (psoriasin) is induced by the proinflammatory cytokines oncostatin- $\mathrm{M}$ and interleukin-6 in human breast cancer. Oncogene. 2010;29(14):2083-92. doi: 10.1038/onc.2009.488. PubMed PMID: 20101226.

109. Jorcyk CL, Holzer RG, Ryan RE. Oncostatin M induces cell detachment and enhances the metastatic capacity of T-47D human breast carcinoma cells. Cytokine. 2006;33(6):323-36. doi: DOI: 10.1016/j.cyto.2006.03.004.

110. West NR, Murphy LC, Watson PH. Oncostatin M suppresses oestrogen receptor- $\alpha$ expression and is associated with poor outcome in human breast cancer. Endocr Relat Cancer. 2012;19(2):181-95. doi: 10.1530/erc-11-0326. PubMed PMID: 22267707.

111. Murray JI, West NR, Murphy LC, Watson PH. Intratumoural inflammation and endocrine resistance in breast cancer. Endocr Relat Cancer. 2015;22(1):R51-67. Epub 2014/11/17. doi: 10.1530/ERC-14-0096. PubMed PMID: 25404688 .

112. Rowan AD, Koshy PJ, Shingleton WD, Degnan BA, Heath JK, Vernallis AB, Spaull JR, Life PF, Hudson K, Cawston TE. Synergistic effects of glycoprotein 130 binding cytokines in combination with interleukin-1 on cartilage collagen breakdown. Arthritis Rheum. 2001;44(7):1620-32. doi: 10.1002/1529-0131(200107)44:7<1620::AIDART285>3.0.CO;2-B. PubMed PMID: 11465713.

113. Boulanger MJ, Chow DC, Brevnova EE, Garcia KC. Hexameric structure and assembly of the interleukin-6/IL-6 alpha-receptor/gp130 complex. Science. 2003;300(5628):2101-4. doi: 10.1126/science.1083901. PubMed PMID: 12829785.

114. Ogata A, Chauhan D, Teoh G, Treon SP, Urashima M, Schlossman RL, Anderson KC. IL-6 triggers cell growth via the Ras-dependent mitogen-activated protein kinase cascade. J Immunol. 1997;159(5):2212-21. PubMed PMID: 9278309.

115. Heinrich PC, Behrmann I, Haan S, Hermanns HM, Müller-Newen G, Schaper F. Principles of interleukin (IL)-6-type cytokine signalling and its regulation. Biochem J. 2003;374(Pt 1):1-20. doi: 10.1042/bj20030407. PubMed PMID: 12773095; PMCID: PMC1223585.

116. Yang X, Lin A, Jiang N, Yan H, Ni Z, Qian J, Fang W. Interleukin-6 trans-signalling induces vascular endothelial growth factor synthesis partly via Janus 
kinases-STAT3 pathway in human mesothelial cells. Nephrology (Carlton). 2017;22(2):150-8. doi: 10.1111/nep.12746. PubMed PMID: 26869278.

117. Asgeirsson KS, Olafsdóttir K, Jónasson JG, Ogmundsdóttir HM. The effects of IL-6 on cell adhesion and e-cadherin expression in breast cancer. Cytokine. 1998;10(9):720-8. doi: 10.1006/cyto.1998.0349. PubMed PMID: 9770334.

118. Chang Q, Bournazou E, Sansone P, Berishaj M, Gao SP, Daly L, Wels J, Theilen T, Granitto S, Zhang X, Cotari J, Alpaugh ML, de Stanchina E, Manova K, Li M, Bonafe M, Ceccarelli C, Taffurelli M, Santini D, Altan-Bonnet G, Kaplan R, Norton L, Nishimoto N, Huszar D, Lyden D, Bromberg J. The IL-6/JAK/Stat3 feed-forward loop drives tumorigenesis and metastasis. Neoplasia. 2013;15(7):848-62. PubMed PMID: 23814496 ; PMCID: PMC3689247.

119. Heo TH, Wahler J, Suh N. Potential therapeutic implications of IL-6/IL6R/gp130-targeting agents in breast cancer. Oncotarget. 2016;7(13):15460-73. doi: 10.18632/oncotarget.7102. PubMed PMID: 26840088; PMCID: PMC4941253.

120. van Rhee F, Wong RS, Munshi N, Rossi JF, Ke XY, Fosså A, Simpson D, Capra M, Liu T, Hsieh RK, Goh YT, Zhu J, Cho SG, Ren H, Cavet J, Bandekar R, Rothman M, Puchalski TA, Reddy M, van de Velde H, Vermeulen J, Casper C. Siltuximab for multicentric Castleman's disease: a randomised, double-blind, placebocontrolled trial. Lancet Oncol. 2014;15(9):966-74. Epub 2014/07/17. doi: 10.1016/S1470-2045(14)70319-5. PubMed PMID: 25042199.

121. Galeotti C, Boucheron A, Guillaume S, Koné-Paut I. Sustained remission of multicentric Castleman disease in children treated with tocilizumab, an antiinterleukin-6 receptor antibody. Mol Cancer Ther. 2012;11(8):1623-6. Epub 2012/05/25. doi: 10.1158/1535-7163.MCT-11-0972. PubMed PMID: 22638145.

122. Hudes G, Tagawa ST, Whang YE, Qi M, Qin X, Puchalski TA, Reddy M, Cornfeld M, Eisenberger M. A phase 1 study of a chimeric monoclonal antibody against interleukin-6, siltuximab, combined with docetaxel in patients with metastatic castrationresistant prostate cancer. Invest New Drugs. 2013;31(3):669-76. Epub 2012/07/25. doi: 10.1007/s10637-012-9857-z. PubMed PMID: 22828917.

123. Angevin E, Tabernero J, Elez E, Cohen SJ, Bahleda R, van Laethem JL, Ottensmeier C, Lopez-Martin JA, Clive S, Joly F, Ray-Coquard I, Dirix L, Machiels JP, Steven N, Reddy M, Hall B, Puchalski TA, Bandekar R, van de Velde H, Tromp B, Vermeulen J, Kurzrock R. A phase I/II, multiple-dose, dose-escalation study of siltuximab, an anti-interleukin-6 monoclonal antibody, in patients with advanced solid tumors. Clin Cancer Res. 2014;20(8):2192-204. Epub 2014/02/21. doi: 10.1158/10780432.CCR-13-2200. PubMed PMID: 24563479.

124. Oh K, Lee OY, Park Y, Seo MW, Lee DS. IL-1 $\beta$ induces IL-6 production and increases invasiveness and estrogen-independent growth in a TG2-dependent manner in human breast cancer cells. BMC Cancer. 2016;16(1):724. Epub 2016/09/08. doi: 10.1186/s12885-016-2746-7. PubMed PMID: 27609180; PMCID: PMC5017052.

125. Dagenais M, Dupaul-Chicoine J, Douglas T, Champagne C, Morizot A, Saleh M. The Interleukin (IL)-1R1 pathway is a critical negative regulator of PyMTmediated mammary tumorigenesis and pulmonary metastasis. Oncoimmunology. 2017;6(3):e1287247. Epub 2017/02/06. doi: 10.1080/2162402X.2017.1287247. PubMed PMID: 28405519; PMCID: PMC5384409. 
126. Risbud MV, Shapiro IM. Role of cytokines in intervertebral disc degeneration: pain and disc content. Nat Rev Rheumatol. 2014;10(1):44-56. Epub 2013/10/29. doi: 10.1038/nrrheum.2013.160. PubMed PMID: 24166242; PMCID: PMC4151534.

127. Kwon G, Corbett JA, Rodi CP, Sullivan P, McDaniel ML. Interleukin-1 beta-induced nitric oxide synthase expression by rat pancreatic beta-cells: evidence for the involvement of nuclear factor kappa B in the signaling mechanism. Endocrinology. 1995;136(11):4790-5. doi: 10.1210/endo.136.11.7588208. PubMed PMID: 7588208.

128. Yoshida Y, Kumar A, Koyama Y, Peng H, Arman A, Boch JA, Auron PE. Interleukin 1 activates STAT3/nuclear factor-kappaB cross-talk via a unique TRAF6- and p65-dependent mechanism. J Biol Chem. 2004;279(3):1768-76. Epub 2003/10/30. doi: 10.1074/jbc.M311498200. PubMed PMID: 14593105.

129. Raymond L, Eck S, Mollmark J, Hays E, Tomek I, Kantor S, Elliott S, Vincenti M. Interleukin-1 beta induction of matrix metalloproteinase-1 transcription in chondrocytes requires ERK-dependent activation of CCAAT enhancer-binding proteinbeta. J Cell Physiol. 2006;207(3):683-8. doi: 10.1002/jcp.20608. PubMed PMID: 16453302.

130. Apte RN, Dotan S, Elkabets M, White MR, Reich E, Carmi Y, Song X, Dvozkin T, Krelin Y, Voronov E. The involvement of IL-1 in tumorigenesis, tumor invasiveness, metastasis and tumor-host interactions. Cancer Metastasis Rev. 2006;25(3):387-408. doi: 10.1007/s 10555-006-9004-4. PubMed PMID: 17043764.

131. Voronov E, Dotan S, Krelin Y, Song X, Elkabets M, Carmi Y, Rider P, Idan Cohen, Romzova M, Kaplanov I, Apte RN. Unique Versus Redundant Functions of IL- $1 \alpha$ and IL-1 $\beta$ in the Tumor Microenvironment. Front Immunol. 2013;4:177. Epub 2013/07/08. doi: 10.3389/fimmu.2013.00177. PubMed PMID: 23847618; PMCID: PMC3703603.

132. Holen I, Lefley DV, Francis SE, Rennicks S, Bradbury S, Coleman RE, Ottewell P. IL-1 drives breast cancer growth and bone metastasis in vivo. Oncotarget. 2016;7(46):75571-84. doi: 10.18632/oncotarget.12289. PubMed PMID: 27765923; PMCID: PMC5342762.

133. Mon NN, Senga T, Ito S. Interleukin-1 $\beta$ activates focal adhesion kinase and Src to induce matrix metalloproteinase-9 production and invasion of MCF-7 breast cancer cells. Oncol Lett. 2017;13(2):955-60. Epub 2016/12/20. doi: 10.3892/ol.2016.5521. PubMed PMID: 28356984; PMCID: PMC5351262.

134. Dinarello CA. Why not treat human cancer with interleukin-1 blockade? Cancer Metastasis Rev. 2010;29(2):317-29. doi: 10.1007/s10555-010-9229-0. PubMed PMID: 20422276; PMCID: PMC2865633.

135. Lubberink M, Golla SS, Jonasson M, Rubin K, Glimelius B, Sörensen J, Nygren P. (15)O-Water PET Study of the Effect of Imatinib, a Selective Platelet-Derived Growth Factor Receptor Inhibitor, Versus Anakinra, an IL-1R Antagonist, on WaterPerfusable Tissue Fraction in Colorectal Cancer Metastases. J Nucl Med. 2015;56(8):1144-9. Epub 2015/06/11. doi: 10.2967/jnumed.114.151894. PubMed PMID: 26069310.

136. Housby JN, Cahill CM, Chu B, Prevelige R, Bickford K, Stevenson MA, Calderwood SK. Non-steroidal anti-inflammatory drugs inhibit the expression of 
cytokines and induce HSP70 in human monocytes. Cytokine. 1999;11(5):347-58. doi: 10.1006/cyto.1998.0437. PubMed PMID: 10328874.

137. Syggelos SA, Giannopoulou E, Gouvousis PA, Andonopoulos AP, Aletras AJ, Panagiotopoulos E. In vitro effects of non-steroidal anti-inflammatory drugs on cytokine, prostanoid and matrix metalloproteinase production by interface membranes from loose hip or knee endoprostheses. Osteoarthritis Cartilage. 2007;15(5):531-42. Epub 2006/12/22. doi: 10.1016/j.joca.2006.11.003. PubMed PMID: 17188523.

138. Gallelli L, Galasso O, Falcone D, Southworth S, Greco M, Ventura V, Romualdi P, Corigliano A, Terracciano R, Savino R, Gulletta E, Gasparini G, De Sarro G. The effects of nonsteroidal anti-inflammatory drugs on clinical outcomes, synovial fluid cytokine concentration and signal transduction pathways in knee osteoarthritis. A randomized open label trial. Osteoarthritis Cartilage. 2013;21(9):1400-8. doi:

10.1016/j.joca.2013.06.026. PubMed PMID: 23973155.

139. Djuric S, Winkler J, Glaser K. Therapeutic Regulation of Inflammation and Angiogenesis in Cancer, Arthritis, and Asthma, Sixth Annual Midwest Meeting, Chicago, IL, USA, March 16, 1998. Inflamm Res. 1999;48(3):101-3. doi:

10.1007/s000110050430. PubMed PMID: 10219650.

140. Bian XW, Chen JH, Jiang XF, Bai JS, Wang QL, Zhang X. Angiogenesis as an immunopharmacologic target in inflammation and cancer. Int Immunopharmacol. 2004;4(12):1537-47. doi: 10.1016/j.intimp.2004.07.017. PubMed PMID: 15351323.

141. Dalgleish AG, O'Byrne K. Inflammation and cancer: the role of the immune response and angiogenesis. Cancer Treat Res. 2006;130:1-38. PubMed PMID: 16610701.

142. Waldner MJ, Wirtz S, Jefremow A, Warntjen M, Neufert C, Atreya R, Becker C, Weigmann B, Vieth M, Rose-John S, Neurath MF. VEGF receptor signaling links inflammation and tumorigenesis in colitis-associated cancer. J Exp Med. 2010;207(13):2855-68. Epub 2010/11/22. doi: 10.1084/jem.20100438. PubMed PMID: 21098094; PMCID: PMC3005238.

143. Peng G, Liu Y. Hypoxia-inducible factors in cancer stem cells and inflammation. Trends Pharmacol Sci. 2015;36(6):374-83. Epub 2015/04/06. doi: 10.1016/j.tips.2015.03.003. PubMed PMID: 25857287; PMCID: PMC4461458.

144. Triner D, Shah YM. Hypoxia-inducible factors: a central link between inflammation and cancer. J Clin Invest. 2016;126(10):3689-98. Epub 2016/08/15. doi: 10.1172/JCI84430. PubMed PMID: 27525434; PMCID: PMC5096825.

145. Kuschel A, Simon P, Tug S. Functional regulation of HIF-1 $\alpha$ under normoxia--is there more than post-translational regulation? J Cell Physiol. 2012;227(2):514-24. doi: 10.1002/jcp.22798. PubMed PMID: 21503885.

146. Damert A, Ikeda E, Risau W. Activator-protein-1 binding potentiates the hypoxia-induciblefactor-1-mediated hypoxia-induced transcriptional activation of vascular-endothelial growth factor expression in C6 glioma cells. Biochem J. 1997;327 ( Pt 2):419-23. PubMed PMID: 9359410; PMCID: PMC1218810.

147. Brat DJ, Kaur B, Van Meir EG. Genetic modulation of hypoxia induced gene expression and angiogenesis: relevance to brain tumors. Front Biosci. 2003;8:d10016. Epub 2003/01/01. PubMed PMID: 12456339.

148. Vollmer S, Kappler V, Kaczor J, Flügel D, Rolvering C, Kato N, Kietzmann T, Behrmann I, Haan C. Hypoxia-inducible factor 1alpha is up-regulated by 
oncostatin $M$ and participates in oncostatin M signaling. Hepatology. 2009;50(1):253-60. doi: 10.1002/hep.22928. PubMed PMID: 19441100.

149. Fossey SL, Bear MD, Kisseberth WC, Pennell M, London CA. Oncostatin M promotes STAT3 activation, VEGF production, and invasion in osteosarcoma cell lines. BMC Cancer. 2011;11:125. doi: 1471-2407-11-125 [pii]

10.1186/1471-2407-11-125. PubMed PMID: 21481226; PMCID: PMC3079692.

150. Weiss TW, Simak R, Kaun C, Rega G, Pflüger H, Maurer G, Huber K, Wojta J. Oncostatin M and IL-6 induce u-PA and VEGF in prostate cancer cells and correlate in vivo. Anticancer Res. 2011;31(10):3273-8. PubMed PMID: 21965736.

151. Aalders KC, Tryfonidis K, Senkus E, Cardoso F. Anti-angiogenic treatment in breast cancer: Facts, successes, failures and future perspectives. Cancer Treat Rev. 2017;53:98-110. Epub 2017/01/03. doi: 10.1016/j.ctrv.2016.12.009. PubMed PMID: 28088074.

152. Bednarz-Knoll N, Alix-Panabieres C, Pantel K. Clinical relevance and biology of circulating tumor cells. Breast Cancer Res. 2011;13(6):228. doi: 10.1186/bcr2940. PubMed PMID: 22114869; PMCID: 3326546.

153. Cohen SJ, Punt CJ, Iannotti N, Saidman BH, Sabbath KD, Gabrail NY, Picus J, Morse M, Mitchell E, Miller MC, Doyle GV, Tissing H, Terstappen LW, Meropol NJ. Relationship of circulating tumor cells to tumor response, progression-free survival, and overall survival in patients with metastatic colorectal cancer. J Clin Oncol. 2008;26(19):3213-21. doi: 10.1200/JCO.2007.15.8923. PubMed PMID: 18591556.

154. de Bono JS, Scher HI, Montgomery RB, Parker C, Miller MC, Tissing H, Doyle GV, Terstappen LW, Pienta KJ, Raghavan D. Circulating tumor cells predict survival benefit from treatment in metastatic castration-resistant prostate cancer. Clin Cancer Res. 2008;14(19):6302-9. doi: 10.1158/1078-0432.CCR-08-0872. PubMed PMID: 18829513.

155. Zhao S, Yang H, Zhang M, Zhang D, Liu Y, Liu Y, Song Y, Zhang X, Li H, Ma W, Zhang Q. Circulating tumor cells (CTCs) detected by triple-marker EpCAM, CK19, and hMAM RT-PCR and their relation to clinical outcome in metastatic breast cancer patients. Cell Biochem Biophys. 2013;65(2):263-73. doi: 10.1007/s12013-0129426-2. PubMed PMID: 22990361.

156. Gradilone A, Raimondi C, Nicolazzo C, Petracca A, Gandini O, Vincenzi B, Naso G, Agliano AM, Cortesi E, Gazzaniga P. Circulating tumour cells lacking cytokeratin in breast cancer: the importance of being mesenchymal. J Cell Mol Med. 2011;15(5):1066-70. doi: 10.1111/j.1582-4934.2011.01285.x. PubMed PMID: 21352474.

157. Gorges TM, Tinhofer I, Drosch M, Rose L, Zollner TM, Krahn T, von Ahsen O. Circulating tumour cells escape from EpCAM-based detection due to epithelial-to-mesenchymal transition. BMC Cancer. 2012;12:178. doi: 10.1186/14712407-12-178. PubMed PMID: 22591372; PMCID: 3502112.

158. Marrinucci D, Bethel K, Kolatkar A, Luttgen MS, Malchiodi M, Baehring F, Voigt K, Lazar D, Nieva J, Bazhenova L, Ko AH, Korn WM, Schram E, Coward M, Yang X, Metzner T, Lamy R, Honnatti M, Yoshioka C, Kunken J, Petrova Y, Sok D, Nelson D, Kuhn P. Fluid biopsy in patients with metastatic prostate, pancreatic and breast cancers. Phys Biol. 2012;9(1):016003. Epub 2012/02/03. doi: 10.1088/1478-

3975/9/1/016003. PubMed PMID: 22306768; PMCID: PMC3387996. 
159. Ross AA, Cooper BW, Lazarus HM, Mackay W, Moss TJ, Ciobanu N, Tallman MS, Kennedy MJ, Davidson NE, Sweet D. Detection and viability of tumor cells in peripheral blood stem cell collections from breast cancer patients using immunocytochemical and clonogenic assay techniques. Blood. 1993;82(9):2605-10. PubMed PMID: 8219214.

160. Schneider T, Osl F, Friess T, Stockinger H, Scheuer WV. Quantification of human Alu sequences by real-time PCR--an improved method to measure therapeutic efficacy of anti-metastatic drugs in human xenotransplants. Clin Exp Metastasis. 2002;19(7):571-82. PubMed PMID: 12498386.

161. Cristofanilli M, Hayes DF, Budd GT, Ellis MJ, Stopeck A, Reuben JM, Doyle GV, Matera J, Allard WJ, Miller MC, Fritsche HA, Hortobagyi GN, Terstappen LW. Circulating tumor cells: a novel prognostic factor for newly diagnosed metastatic breast cancer. J Clin Oncol. 2005;23(7):1420-30. doi: 10.1200/JCO.2005.08.140. PubMed PMID: 15735118.

162. Bolin C, Sutherland C, Tawara K, Moselhy J, Jorcyk CL. Novel mouse mammary cell lines for in vivo bioluminescence imaging (BLI) of bone metastasis. Biol Proced Online. 2012;14(1):6. Epub 2012/04/17. doi: 10.1186/1480-9222-14-6. PubMed PMID: 22510147; PMCID: PMC3473320.

163. Yuan L, Liu X. Platelets are associated with xenograft tumor growth and the clinical malignancy of ovarian cancer through an angiogenesis-dependent mechanism. Mol Med Rep. 2015;11(4):2449-58. Epub 2014/12/11. doi: 10.3892/mmr.2014.3082. PubMed PMID: 25502723; PMCID: PMC4337475.

164. Hutchinson JN, Muller WJ. Transgenic mouse models of human breast cancer. Oncogene. 2000;19(53):6130-7. doi: 10.1038/sj.onc.1203970. PubMed PMID: 11156526.

165. Lelekakis M, Moseley JM, Martin TJ, Hards D, Williams E, Ho P, Lowen D, Javni J, Miller FR, Slavin J, Anderson RL. A novel orthotopic model of breast cancer metastasis to bone. Clin Exp Metastasis. 1999;17(2):163-70. PubMed PMID: 10411109.

166. Holen I, Speirs V, Morrissey B, Blyth K. In vivo models in breast cancer research: progress, challenges and future directions. Dis Model Mech. 2017;10(4):35971. doi: 10.1242/dmm.028274. PubMed PMID: 28381598; PMCID: PMC5399571.

167. Rhodes DR, Kalyana-Sundaram S, Mahavisno V, Varambally R, Yu J, Briggs BB, Barrette TR, Anstet MJ, Kincead-Beal C, Kulkarni P, Varambally S, Ghosh D, Chinnaiyan AM. Oncomine 3.0: genes, pathways, and networks in a collection of 18,000 cancer gene expression profiles. Neoplasia. 2007;9(2):166-80. PubMed PMID: 17356713; PMCID: PMC1813932.

168. Gao J, Aksoy BA, Dogrusoz U, Dresdner G, Gross B, Sumer SO, Sun Y, Jacobsen A, Sinha R, Larsson E, Cerami E, Sander C, Schultz N. Integrative analysis of complex cancer genomics and clinical profiles using the cBioPortal. Sci Signal. 2013;6(269):pl1. Epub 2013/04/02. doi: 10.1126/scisignal.2004088. PubMed PMID: 23550210 ; PMCID: PMC4160307.

169. Uhlén M, Fagerberg L, Hallström BM, Lindskog C, Oksvold P, Mardinoglu A, Sivertsson A, Kampf C, Sjöstedt E, Asplund A, Olsson I, Edlund K, Lundberg E, Navani S, Szigyarto CA, Odeberg J, Djureinovic D, Takanen JO, Hober S, Alm T, Edqvist PH, Berling H, Tegel H, Mulder J, Rockberg J, Nilsson P, Schwenk JM, Hamsten M, von Feilitzen K, Forsberg M, Persson L, Johansson F, Zwahlen M, von 
Heijne G, Nielsen J, Pontén F. Proteomics. Tissue-based map of the human proteome. Science. 2015;347(6220):1260419. doi: 10.1126/science.1260419. PubMed PMID: 25613900.

170. Forbes SA, Beare D, Boutselakis H, Bamford S, Bindal N, Tate J, Cole CG, Ward S, Dawson E, Ponting L, Stefancsik R, Harsha B, Kok CY, Jia M, Jubb H, Sondka Z, Thompson S, De T, Campbell PJ. COSMIC: somatic cancer genetics at highresolution. Nucleic Acids Res. 2017;45(D1):D777-D83. Epub 2016/11/28. doi:

10.1093/nar/gkw1121. PubMed PMID: 27899578; PMCID: PMC5210583.

171. Ritts RE, Del Villano BC, Go VL, Herberman RB, Klug TL, Zurawski VR. Initial clinical evaluation of an immunoradiometric assay for CA 19-9 using the NCI serum bank. Int J Cancer. 1984;33(3):339-45. PubMed PMID: 6199316.

172. QIAGEN. Precancerous inflammation can cause increased genetic and epigenetic damage 2017 [cited 2017 July]. Available from:

https://www.qiagen.com/ /media/nextq/image\%20library/illu/03/50/illu_0350_rt2profiler /1_8.ashx?h=1243\&amp;amp;la=en\&amp;amp;w=800\&amp;amp;hash=070ADF703315 6B53C892EAF28D2638912A60D2A9.

173. RnCeus. Histology of DCISHistology of DCIS 2017 [cited 2017 July]. Available from: http://www.rnceus.com/dcis/image\%20folder/DCISrange.jpg.

174. Brown LF, Dvorak AM, Dvorak HF. Leaky vessels, fibrin deposition, and fibrosis: a sequence of events common to solid tumors and to many other types of disease. Am Rev Respir Dis. 1989;140(4):1104-7. doi: 10.1164/ajrccm/140.4.1104. PubMed PMID: 2478057.

175. Lungevity. Blood Vessel Overgrowth on Cell 2017 [cited 2017 July]. Available from: https://www.lungevity.org/sites/default/files/illustrations/blood-vesselovergrowth.png. 
CHAPTER TWO: CO-EXPRESSION OF VEGF AND IL-6 FAMILY CYTOKINES ARE ASSOCIATED WITH DECREASED SURVIVAL IN HER2- BREAST CANCER: SUBTYPE SPECIFIC IL-6 FAMILY CYTOKINE-MEDIATED VEGF SECRETION

\author{
Authors \\ Ken Tawara ${ }^{1}$, Hannah Scott $^{2}$, Jacqueline Emathinger ${ }^{2}$, Alex $\operatorname{Ide}^{2}$, Ryan Fox ${ }^{2}$, Dollie \\ LaJoie $^{2,3}$, Danielle Hedeen ${ }^{2,3}$, Madhuri Nandakumar ${ }^{2}$, Andrew J. Oler ${ }^{2,4}$, Ryan Holzer ${ }^{2,5}$, \\ and Cheryl Jorcyk ${ }^{1,2, \#}$ \\ ${ }^{1}$ Boise State University, Biomolecular Sciences Program, 1910 University Drive, \\ Boise, ID \\ ${ }^{2}$ Boise State University, Department of Biological Sciences, 1910 University \\ Drive, Boise, ID \\ ${ }^{3}$ University of Utah, Department of Oncological Sciences, Salt Lake City, UT \\ ${ }^{4}$ Bioinformatics and Computational Biosciences Branch, Office of Cyber \\ Infrastructure and Computational Biology, NIAID/NIH, Bethesda, Maryland, USA \\ ${ }^{5}$ Rosetta Institute of Biomedical Research, San Jose, CA

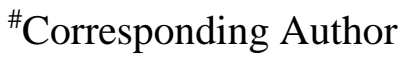




\begin{abstract}
Background: Breast cancer cell-response to inflammatory cytokines such as interleukin-6 (IL-6) and oncostatin M (OSM) may affect the course of clinical disease in a cancer subtype-dependent manner. Furthermore, vascular endothelial growth factor A (VEGF) secretion induced by IL-6 and OSM may also be subtype-dependent. Here we investigate the relationship between cytokine signaling, patient survival, and VEGF-A in invasive ductal carcinoma (IDC).

Methods: The association between expression of cytokines, their receptors, and VEGF was assessed with patient survival in a breast cancer subtype-specific manner using datasets from Oncomine $\mathrm{TM}^{\mathrm{TM}}$. To assess specific signaling factors involved in cytokine-induced VEGF secretion, siRNAs for hypoxia inducible factor $1 \alpha(\mathrm{HIF} 1 \alpha)$ and signal transducer and activator of transcription 3 (STAT3) were used. The levels of VEGF secretion were then analyzed by ELISA.

Results: In IDC patients, high VEGF expression in tumors together with high cytokine or cytokine receptor expression correlates with decreased survival. Specifically, patient survival is significantly lower in HER2-, but not in HER2+, disease when tumor co-expression of VEGF and OSM, OSMR $\beta$, IL-6, or IL-6R $\alpha$ is high. Furthermore, assessment of HER2- breast cancer cells in vitro identified unique signaling differences regulating cytokine-induced VEGF secretion. In particular, OSM signals through STAT3 and not HIF1 $\alpha$ to induce VEGF secretion in ER-MDA-MB-231 human breast cancer cells, while the in ER+ T47D cells, OSM signals through both STAT3 and HIF1 $\alpha$. Additionally, ER- MDA-MB-231 cells only respond to OSM and not IL-6, while T47D cells respond to both OSM and IL-6, though to a lesser extent.
\end{abstract}


Conclusions: These results highlight that both the survival of breast cancer patients with high co-expression of VEGF and IL-6 family cytokines, as well as IL-6 family cytokine-mediated VEGF secretion is dependent on specific breast cancer subtype. Thus, the heterogeneity of human breast cancer in relation to IL-6 family cytokines and VEGF may have important implications in clinical treatment options, disease progression, and ultimately patient prognosis.

\section{Introduction}

In the United States, breast cancer is the most frequently diagnosed cancer among women (1). With 252,710 new cases of invasive breast cancer (IBC) expected for 2017, breast cancer remains a leading public health concern, both in the United States and globally. One of the main concerns is the complex relationship between IBC subtypes, clinical treatment, and long-term survival $(1,2)$. The current known IBC subtypes are not able to fully capture the heterogeneous nature of breast cancer, and our current understanding is lacking in terms of patient treatment versus survival (3). In particular, anti-angiogenic therapies for breast cancer have had variable clinical success at best, and at worst, show no improvement in disease free survival $(4,5)$. The high level of clinical variability with anti-angiogenic therapies may be due to the highly heterogeneous nature of breast cancer and their subsequent biomarkers (6). This underlies the need to improve our understanding of clinical outcomes in conjunction with existing breast cancer subtype biomarkers such as estrogen receptor (ER), progesterone receptor (PR), and human epidermal growth factor receptor 2 (HER2).

Current breast cancer subtype classifications depend on the evaluation of ER, PR, and HER2 (3). Luminal A (ER+ PR+ HER2-; low Ki67) and luminal B (ER+ PR+ 
HER2-; high Ki67 or HER2+) breast cancer subtypes are typically less aggressive and display a more favorable prognosis relative to other subtypes (7-9). In contrast, cancers classified as basal-like triple negative breast cancer (TNBC; ER- PR- HER2-) have high frequencies of relapse and display less favorable prognoses, in large part due to the lack of targeted therapies available to treat this subtype $(3,7)$. Similarly, HER2-enriched cancers (ER- PR- HER2+) also have unfavorable prognoses (10). While superficial differences between the subtypes are relatively well known, the specific molecular mechanisms that drive these differences remain elusive. Specifically, increased inflammatory markers in the serum of breast cancer patients appear to be associated with poor prognosis (11).

Chronic inflammation promotes a maladaptive pathological state, in which inflammatory cytokines actually promote tumorigenesis, invasion, and metastasis (12). Cytokines of the interleukin-6 (IL-6) family, including IL-6 and oncostatin M (OSM), have been implicated in the migration and invasiveness of human breast cancer cells (1315), while leukemia inhibitory factor (LIF) has been shown to act as a tumor/metastasis suppressor (16-19). Moreover, both IL-6 and OSM have been shown to be capable of directly and indirectly driving angiogenesis (20-22). Receptor complexes of the IL-6 family all consist of at least one molecule of glycoprotein 130 (gp130), as well as subunit(s) specific to their respective receptor $(23,24)$. Following IL-6 or OSM ligand binding, the gp130 receptor complexes activates the JAK/STAT, MAPK, PI3K/AKT, and JNK pathways, thereby mediating transcription of target genes (25-30). Although IL-6 and LIF bind specifically to their individual receptors (IL-6R and LIFR, respectively), OSM is capable of binding to both the LIFR and the OSM receptor (OSMR) (25, 31-33). 
The resultant cytokine signaling plays various roles in the development of breast cancer, through activation of target genes involved in differentiation, survival, apoptosis, and angiogenesis $(29,32,34)$.

Angiogenesis is controlled by a balance of pro- and anti-angiogenic factors that maintain a normal rate of blood vessel growth (35). During tumor angiogenesis, both tumor cells and tumor-associated stromal/immune cells secrete proangiogenic factors (30, 36). The most potent proangiogenic factor, vascular endothelial growth factor A (VEGFA), promotes the survival, proliferation, and motility of endothelial cells and enhances vascular permeability $(37,38)$. Other VEGF subtypes include the weakly angiogenic VEGF-B, and the lymphangiogenic and vasculogenic VEGF-C and VEGF-D (39, 40). As a proangiogenic factor, VEGF-A (also referred to as VEGF) regulation typically depends on hypoxia-driven signaling through the dimeric transcription factor hypoxia-inducible factor 1 (HIF1). As a dimer, HIF1 consists of the inducible HIF1 $\alpha$ subunit, which when partnered with the constitutively expressed HIF1 $\beta$, binds the hypoxia response element (HRE) (41). However, transcription factor binding sites in the VEGF promoter other than the HRE, such as those for signal transducer and activator of transcription-3 (STAT3) and activator protein 1 (AP1), allow transcription to be activated by numerous pathways under both hypoxic and non-hypoxic conditions (42-45). Under non-hypoxic conditions, IL-6 family cytokines have been shown to promote VEGF expression via activation of transcription factors HIF1 $\alpha$ and STAT3 $(22,30,46,47)$.

In this paper, we study the differential effects of the inflammatory interleukin-6 (IL-6)-family cytokines on breast cancer patient outcomes, as well as the induction of vascular endothelial growth factor A (VEGF) in a breast cancer subtype-specific manner. 
Using Oncomine data, we systemically explored the co-expression of VEGF with inflammatory cytokine components among invasive ductal carcinoma (IDC) patients subdivided by HER2- or HER2+ status. Specifically, we found that HER2- patient survival significantly decreases when breast tumors co-express high levels of VEGF and high levels of OSM, OSMR $\beta$, IL-6, or IL-6R $\alpha$. Conversely, in patients with HER2+ disease, co-expression of VEGF and these inflammatory proteins had little to no effect on survival. Furthermore, through treatment of HER2- breast cancer cell lines with these cytokines, we elucidate some of the specific signaling factors involved in the mediation of VEGF secretion. Interestingly, regulation of IL-6-family cytokine-induced VEGF in HER2- cells differed between ER+ T47D (ER+ PR+ HER2-) and ER- MDA-MB-231 (ER- PR- HER2-) cells. In T47D cells, HIF1 $\alpha$ played a traditional role in transcriptionally activating VEGF secretion, while in MDA-MB-231 cells, VEGF secretion was independent of HIF $1 \alpha$ and dependent on STAT3 signaling. These results highlight the breast cancer subtype-specific differences in cytokine signaling that lead to VEGF secretion, and importantly, the potential for therapeutic suppression of IL-6 family cytokines in HER2- breast cancer.

\section{Materials and Methods}

$\underline{\text { Oncomine analysis }}$

To explore correlations between IL-6 family cytokines, cytokine receptors, VEGF expression, breast cancer receptor status, and patient survival, we attained the Curtis Breast human mRNA microarray dataset from Oncomine ${ }^{\mathrm{TM}}$ (Compendia Bioscience, Ann Arbor, MI). The constraints used to define the dataset used were "Invasive Ductal Carcinoma" and a detailed survival status of either "Alive" or "Dead of Disease." The 
resultant dataset was used to calculate quartiles. From these, the upper quartile $\left(>75^{\text {th }}\right.$ percentile) and lower quartile $\left(<25^{\text {th }}\right.$ percentile) were selected for comparison in order to clearly depict survival trends that may have been otherwise muddled by use of all quartile combinations. For co-expression analysis, we calculated survival curves using patients in the upper quartile of both VEGF and each particular IL-6 family gene ("high/high") and the lower quartile of both ("low/low"). Statistical analyses between survival of two groups was calculated using a log-rank test in GraphPad Prism 5 software ${ }^{*} \mathrm{p}<0.05$, $* * \mathrm{p}<0.01, * * * \mathrm{p}<0.001$

$\underline{\text { Tissue culture }}$

MDA-MB-231 and T47D human breast cancer cells (ATCC) were grown in RPMI medium supplemented with $10 \%$ fetal bovine serum (FBS), $1 \%$ penicillin streptomycin, and $1 \%$ sodium pyruvate and incubated at $37^{\circ} \mathrm{C}$ at $5 \% \mathrm{CO}$. Cytokine treatments were prepared in low serum media (1\% FBS) at $25 \mathrm{ng} / \mathrm{mL}$ recombinant human OSM (Cat\#300-10T, Peprotech), IL-6 (Cat \#200-06, Peprotech), and LIF (Cat\# 300-05, Peprotech) for the indicated time points. Human umbilical vein endothelial cells (HUVECs, GlycoTech, Gaithersburg, MD) were grown in M199 medium supplemented

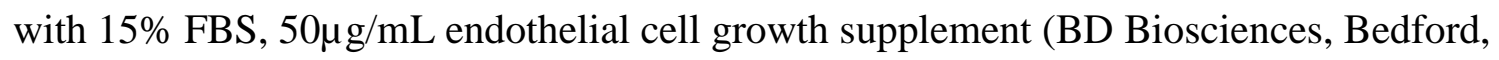
MA) and 10 units $/ \mathrm{mL}$ porcine heparin (Sigma-Aldrich, St. Louis, MO) at $37^{\circ} \mathrm{C}$ in humidified $5 \% \mathrm{CO}_{2}$.

For cytokine treatment, MDA-MB-231 cells were grown to 50\% confluency in RPMI 1640 with 10\% FBS, 1\% Penicillin/Streptomycin and 1\% Sodium Pyruvate. Cells were then starved overnight in serum-free media. Cells were treated with recombinant human OSM (rhOSM, Peprotech Inc., Rocky Hill, NJ) in serum-free RPMI for 72 hours. 
Conditioned media (CM) was collected, cellular debris was removed following centrifugation, and $\mathrm{CM}$ was stored at $-80^{\circ} \mathrm{C}$.

$\underline{\text { siRNA transfection }}$

To determine the mechanism by which VEGF is induced, siRNAs pools targeting HIF1 $\alpha$, STAT3, JNK1 and JNK2 were obtained from Dharmacon. In brief, 300,000 cells/well were plated in a 6-well plate and siRNAs were transfected according to the Fast-Forward protocol as outlined by the manufacturer of Hyperfect siRNA Transfection Reagent (Cat\# 301705, Qiagen). STAT3 siRNAs were used at $25 \mathrm{nM}$ and transfection was incubated for 72 hours, serum starved, and then treated with OSM for up to 48 hours. JNK1 and JNK2 siRNA was used at concentrations of $20 \mathrm{nM}$ and transfected for 24 hours prior to OSM treatment. Knockdown was assessed via immunoblot analysis (see below).

$\underline{\text { Immunoblot analysis }}$

Cells were grown to $50-80 \%$ confluency before being treated with respective cytokines and/or siHIF1 $\alpha$. Cells were lysed on ice with 1x RIPA buffer containing 1x protease inhibitor cocktail (Cat\# P8340, Sigma Aldrich). Lysates were run on an SDSPAGE gel, transferred onto a nitrocellulose membrane, and blocked overnight in 5\% nonfat dry milk in PBS containing 0.05\% Tween 20 (5\% NFDM-PBST). Membranes were incubated overnight in $0.6 \mu \mathrm{g} / \mathrm{mL}$ anti-HIF1 $\alpha$ primary antibody (Dilution: 1:1000 Cat\# AF1935, R\&D Systems) in 5\% NFDM-PBST. Membranes were washed with PBST and incubated with HRP secondary antibody (Cat\# 705-035-003 Jackson ImmunoResearch) in 5\% NFDM-PBST for 45 minutes, developed with ECL, and imaged on X-ray film on a Kodak 4000R Image Station. 
Signaling blots were performed as detailed above with the following antibodies: STAT3 (Cat\# 9132), p-STAT3 (Cat\# 9145), JNK1 (Cat\# 3708), p-JNK1 (Cat\# 4668), $\beta$ Actin (Cat\# 3700) ((1:1000, Cell Signaling Technologies).

\section{VEGF ELISA}

Analysis of VEGF secretion by MDA-MB-231 and T47D cells was assessed via VEGF ELISA according to the manufacturer's protocol (Cat\# Dy293B, R\&D Systems, Bethesda, MD). Cells were plated at a confluency of 50,000 or 100,000 cells in a 24 -well plate and allowed to adhere overnight. The following day, cells were serum-starved in serum free media for 6 hours and treated with cytokines for the indicated time.

Conditioned media was collected and assessed by ELISA. The absorbance was recorded using a microplate reader (Bio-Rad) at $450 \mathrm{~nm}$ and background was subtracted (570 nm). $\underline{\text { RT-PCR }}$

Total RNA was collected from MDA-MB-231 and T47D cells using RNA STAT60 and isolated per the manufacturer's protocol (Tel-Test; Friendswood, TX). cDNA was synthesized according to the manufacturer's protocol (High Capacity cDNA Reverse Transcription Kit, Applied Biosystems, In/Life Technologies). RT-PCR primers used were as follows: OSMR $\beta$ (510 bp): forward, 5'-TTAAAGGCCAGAGGCTATGG-3'; and reverse, 5'-TTGACAGAAGAATTGTGGAA-3'. LIFR (420 bp): forward, 5'ATACAGATGGTGGAGTGG-3'; and reverse, 5'-TGATGGGTGGACAATAGG-3'. IL6R (618 bp): forward, 5'-GTGAGGAAGTTTCAGAACAGTCCG-3'; and reverse, 5'TGGGAGGCTTGTCGCATTTG-3'; gp130 (326 bp): forward, 5'CATGCTTTGGGTGGAATGGAC-3'; and reverse, 5'CATCAACAGGAAGTTGGTCCC-3'; and GAPDH (483 bp): forward, 5'- 
GTCAACGGATTTGGCCGTATT-3'; and reverse, 5'-

AAAGTTGTCATGGATGACCTT-3’

pSTAT3 ELISA

Intracellular pSTAT3 levels were assessed by ELISA in accordance with the manufacturer's protocol (Cat\#7146, Cell Signaling). 50,000 cells were adhered to 24 well plates overnight in serum free media. Cells were then treated with OSM or IL6 (25 $\mathrm{ng} / \mathrm{mL}$ ) for the indicated times, and cell lysates were collected using 1x Cell Lysis Buffer (Cat\# 9803, Cell Signaling). The lysates were diluted 1:3 with blocking buffer (PBS0.05\% Tween 20, $1 \%$ IgG-free BSA) and assessed by ELISA. Absorbance was assessed with a microplate reader at $450 \mathrm{~nm}$ with background correction at $570 \mathrm{~nm}$.

$\underline{\text { Statistical analysis }}$

All statistical analyses were performed using GraphPad Prism 5 software. To compare multiple groups, one- or two-way analysis of variance were run with Tukey's and Bonferroni's post-test, respectively where appropriate on ELISA data. Survival data was depicted as Kaplan-Meier graphs and significance assessed by the Log-rank test. Experiments were considered statistically significant if $p$ values were less than 0.05 . Error bars represent mean \pm S.E. Experiments were performed at least three times to determine statistical significance.

\section{Results}

Inflammatory cytokine and VEGF co-expression is correlated with decreased survival of $\underline{\text { IDC patients }}$

To address the importance of IL-6 family cytokines in invasive ductal carcinoma (IDC), we correlated patient survival relative to expression levels of VEGF with OSM, 
IL-6, LIF, or their specific receptor subunits. Using the Curtis Breast dataset obtained from Oncomine ${ }^{\mathrm{TM}}$ (48), co-expression in IDC patients was compared to survival and quantified by individual quartiles, as demonstrated for OSM and VEGF (Fig. S1A) and OSMR $\beta$ and VEGF (Fig. S1B). Utilizing just the upper and lower quartiles, we observed a significant correlation between high co-expression of OSM and VEGF and decreased survival, relative to low expression of both OSM and VEGF (p=0.0190, Fig. 1A). Similarly, decreased survival was observed in patients who had high expression levels of OSMR $\beta$ and VEGF (p=0.0012, Fig. 1B), IL-6 and VEGF (p=0.0005, Fig. 1C), or IL6R $\alpha$ and VEGF (p=0.0016, Fig. 1D) relative to those with low co-expression of each respective gene pair. Patient survival was not statistically affected by co-expression levels of LIF and VEGF (p=0.0578, Fig. 1E) or LIFR $\beta$ and VEGF (p=0.1020, Fig. 1F). Together, these results highlight the importance of tumor cell co-expression of VEGF with OSM, OSMR $\beta$, IL-6, or IL-6R $\alpha$ on the poor survival of individuals with IDC. HER2- status dictates poor survival in IDC patients with high co-expression of OSM, IL$\underline{6, \text { OSMR } \beta \text { or IL-6R } \alpha \text { and VEGF }}$

As published studies have demonstrated the importance of receptor status in breast cancer recurrence and treatment (49-51), we next analyzed HER2 status on the survival of IDC patients according to VEGF and IL-6 family molecule co-expression. Interestingly, high co-expression of VEGF with OSM, OSMR $\beta$, IL-6, or IL-6R $\alpha$ correlated with the poor survival of HER2- patients but not HER2+ patients (Fig. 2). Specifically, statistically significant decrease in survival was only observed in HER2patients with high OSM and VEGF expression $(\mathbf{p = 0 . 0 0 1 6})$ and not in HER2+ patients (p=0.5963, Fig. 2A). Decreased survival was also seen in HER2- individuals with high 
OSMR $\beta$ and VEGF co-expression (p=0.0001, Fig. 2B), high IL-6 and VEGF coexpression (p=0.0005, Fig. S2A), and high IL-6R $\alpha$ and VEGF co-expression $(\mathbf{p = 0 . 0 1 1 2 ;}$

Fig. S2B). No statistically significant change in survival was detected with the respective HER2+ individuals (High OSMRß/VEGF, p=0.9317, Fig.2B) (High IL-6/VEGF, p=0.6106, Fig. s2A) (High IL-6Ra/VEGF, p=0.0671, Fig S2B). Counter intuitive to its role as a metastasis suppressor, high expression of LIF with high expression of VEGF also was correlated with poor survival in HER2- (p<0.0001, Fig. S2C) but not HER2+ IDC patients (p=0.2806 Fig S2C). However, no association between HER2 status and survival was observed in IDC patients expressing high levels of LIFR $\beta$ and VEGF $(p=0.1247$ and $p=0.534$, respectively, Fig. S2D $)$.

Among HER2- individuals, published results suggest that ER+ patients have the lowest rates of relapse and a considerably more favorable prognosis than individuals expressing other receptor subtypes (49). Therefore, we examined the co-expression of OSM and VEGF with HER2 status in individuals that did or did not express ER. Surprisingly, in HER2- patients, ER+ status was associated with poor survival in IDC

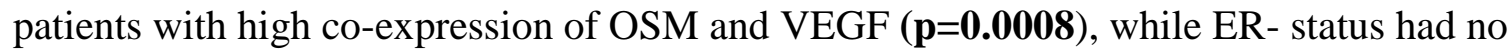
effect on survival (p=0.6998, Fig. S3A, B). ER status played a less significant role in patient survival when HER2 status was associated high OSMR $\beta$ and VEGF coexpression (Fig. S3C, D). These data suggest that while ER+ HER2- patients typically have fair prognoses (49), high tumor co-expression of OSM and VEGF greatly impacts survival in these individuals. 
OSM mediates VEGF induction independent of HIF1 $\alpha$ signaling in TNBC cells

Stark differences are evident when analyzing the effects of cytokine-driven angiogenesis on patient survival, thus underling the heterogeneity of cancer subtypes. As our data suggested, high OSM co-expression with the proangiogenic factor VEGF resulted in poor HER2- patient survival. To uncover mechanistic differences among breast tumor lines with differing ER status, we used two distinct HER2- cell lines, the ER-negative MDA-MB-231 (ER- PR- HER2-) TNBC cell line and the ER-positive T47D (ER+ PR+ HER2-) cell line, to study VEGF induction by IL-6 family cytokines.

Inflammatory cytokines have been shown to promote hypoxia-like effects thereby inducing transcriptional activity of HIF $1 \alpha$, an important transcription factor for hypoxiarelated proteins such as VEGF. Treatment with OSM, IL-6, or LIF (25 ng/mL) induced HIF $1 \alpha$ expression by Western blot analysis in both MDA-MB-231 cells at 24 hours and in T47D cells at 72 hours, relative to non-treated cells (Fig. 3A, B). These time points selected as they were determined to have maximal HIF $1 \alpha$-induction in each cell line (data not shown). The addition of an siRNA targeting HIF $1 \alpha(\operatorname{siHIF} 1 \alpha)$ inhibited the induction of HIF $1 \alpha$ following treatment with inflammatory cytokines. Interestingly, despite the induction of HIF $1 \alpha$ by each of the inflammatory cytokines assessed, an increase in VEGF secretion was observed only in cells treated with OSM. OSM treatment accounted for a 3-fold increase in VEGF secretion in MDA-MB-231 cells at 24 hours $(\mathbf{p}<\mathbf{0 . 0 5})$ and an almost 4-fold increase in T47D cells at 72 hours $(\mathbf{p}<\mathbf{0 . 0 0 1})$ compared to non-treated cells, as assessed by ELISA (Fig. 3C, D). Treatment with IL-6 or LIF did not produce a significant change in the level of VEGF secreted. To determine if VEGF induction was mediated through cytokine-induced HIF $1 \alpha$, VEGF secretion was assessed in the presence 
of cytokine and siHIF $1 \alpha$ together. Relative VEGF secretion was not altered in MDA-MB-231 cells treated with siHIF $1 \alpha$ and OSM (p<0.01, Fig. 3C) relative to untreated control, while this same treatment reduced VEGF secretion by nearly 50\% in T47D cells ( $\mathbf{p}<\mathbf{0 . 0 0 1}$ Fig. 3D). These results demonstrate that OSM-mediated induction of VEGF is at least partially dependent on HIF1 $\alpha$ signaling in T47D cells, yet is independent of HIF $1 \alpha$ signaling in MDA-MB-231 cells. Together, this data suggests that OSM-induced VEGF induction is likely mediated through different pathways in ER+ versus ER- cells.

To confirm a functional effect for OSM-induced VEGF production, we performed in vitro and in vivo angiogenesis assays. Conditioned media (CM) from MDA-MB-231 cells treated with OSM induced endothelial cell tube and branch point formation, which was suppressed by the addition of a VEGF neutralizing antibody (Fig. S4A, B). A similar effect was observed in vivo using a Matrigel plug assay. CM from OSM-treated MDAMB-231 cells was mixed with Matrigel and injected subcutaneously in athymic female mice resulting in a 12-fold increase in angiogenesis, as compared to CM from untreated MDA-MB-231 cells (p<0.001, Fig. S5A, B). Together, these results suggest that OSM, but not IL-6 or LIF, promote the secretion of a functional VEGF from breast cancer cells. OSM strongly induces STAT3 activation in ER+ and ER- HER2- breast cancer cells

OSM is capable of binding to and activating both the OSMR and the LIFR, while LIF binds to only the LIFR and IL-6 binds to the IL-6 receptor. Stimulation of these receptors prompts activation of various signaling pathways including the STAT3 and JNK pathways $(23,52-54)$. To confirm that these receptors were present in our cell lines, mRNA from MDA-MB-231 and T47D cells was assessed by RT-PCR and shown to 
express OSMR $\beta$, LIFR $\beta$, IL-6R $\alpha$, and gp130 (Fig. 4A). Next, we sought to determine the mechanism by which OSM induces the expression of VEGF by investigating the activation of the transcription factors STAT3 and JNK, found downstream of OSMR. Treatment with OSM (25 ng/mL) strongly induced phosphorylation of STAT3 (pSTAT3) in MDA-MB-231 TNBC cells at both 15 and 30 minutes, as assessed by immunoblot. In contrast, addition of OSM only slightly induced phosphorylation of JNK1 (pJNK1) in these cells (Fig. 4B). Neither the addition of IL-6 nor LIF led to STAT3 or JNK1 phosphorylation in ER- MDA-MB-231 cells. In T47D cells, stimulation with both OSM and IL-6 promoted phosphorylation of STAT3 at 15 and 30 minutes (Fig. 4C). Phosphorylation of STAT3 was not induced in ER+ T47D cells treated with LIF. A slight induction of JNK1 phosphorylation was observed following treatment with OSM in T47D cells, whereas IL-6 and LIF did not produce an effect.

In both cell lines, levels of OSM-induced STAT3 phosphorylation were transient yet sustained over time up to 72 hours, with preferential activation around 0.5 hours, relative to treatment with IL-6 (p<0.001, Fig. 4D, E). Interestingly, treatment with IL-6 has no observed effect on pSTAT3 levels in MDA-MB-231 cells and only induced moderate STAT3 phosphorylation in T47D cells. This highlights an important difference between cytokine responsiveness in these cells.

Activation of STAT3 signaling is required for VEGF secretion by MDA-MB-231 TNBC $\underline{\text { cells }}$

As our data suggests, OSM-induced VEGF is independent of HIF1 $\alpha$ in MDAMB-231 TNBC cells. To determine whether OSM promotes VEGF secretion via the STAT3 pathway, we used an siRNA targeting STAT3 (siSTAT3). In MDA-MB-231 
cells, treatment with OSM and siSTAT3 suppressed VEGF secretion to near-baseline levels (Fig. 5A). However, this complete suppression was not observed in T47D cells, likely due to the role of HIF1 $\alpha$ in the secretion of VEGF in this cell line (Fig. 5B). While initial studies using a chemical inhibitor of JNK suggested that JNK signaling was in part necessary for OSM-mediated induction of VEGF (Fig. S6A), further investigations revealed that these effects might have resulted from the off-target suppression of STAT3 phosphorylation (Fig. S6B). However, siRNAs targeting both JNK1 and JNK2 (siJNK1 and siJNK2, respectively) had no effect on the levels of VEGF secretion in MDA-MB231 or T47D cells treated with OSM (Fig. 5C, D). Together, these results demonstrate that OSM mediated induction of VEGF is dependent on STAT3 in MDA-MB-231 cells, while T47D cells utilize both STAT3 and HIF1 $\alpha$ to promote VEGF production in response to OSM.

\section{Discussion}

The different molecular characteristics of human breast cancer have important implications for clinical treatment options, disease progression, and ultimately patient prognosis. Therefore, it is important to understand how molecular mechanisms are impacted by breast cancer subtypes in order to provide patients with comprehensive therapies targeted against their specific disease. In this study, we show the clinical importance of tumor cell co-expression of VEGF with OSM, OSMR $\beta$ IL-6, or IL-6R $\alpha$ on the survival of individuals with HER2- invasive ductal carcinoma. Using this human relevance to drive in vitro studies, we demonstrate that mechanistically, ER+ HER2- and ER- HER2- breast cancer cells respond differently to IL-6 cytokines in their induction of VEGF. 
High tumor expression of the inflammatory cytokine IL-6 has been associated with poor survival in breast as well as cervical cancer patients $(55,56)$. Upregulated OSM, IL-6, and their receptors have been associated with increased metastatic capacity $(15,57)$, including increased invasion and migration $(23,27,28,58,59)$ and the promotion of angiogenesis $(29,30,32,34)$. Moreover, OSM has been shown to bind to extracellular matrix proteins such as collagens, laminins, and fibronectins in an active conformation, thereby accumulating in a breast tumor microenvironment to further promote these effects (60). In the studies presented here, we show that high co-expression of VEGF with OSM, IL-6, or their receptors, correlates with poor survival of IDC patients, suggesting their potential value as negative prognostic markers and therapeutic targets. This association was not observed among patients with high co-expression of VEGF and LIF or LIFR $\beta$, most likely due to the recently described role of LIF as a metastasis suppressor (16-18).

When IDC patients were subdivided by HER2 status, our results demonstrated that overall survival was significantly lower in HER2- patients with high OSM or OSMR $\beta$ and VEGF co-expression than in HER2+ patients. HER2- patients also fared much worse when their tumors had high co-expression of IL-6 or IL-6R $\alpha$ and VEGF. Considering OSM alone, high expression of OSM in HER2- individuals was by itself associated with poor survival, while expression of OSMR $\beta$ was not (data not shown). Taking into consideration VEGF alone, one previous study showed that high VEGF expression was correlated with the presence of axillary nodal metastasis and lower overall survival (OS) rates in non-luminal A breast cancer subtypes, which include HER2- TNBC as well as luminal B and HER2 subtypes (61). Work has also been 
published describing the relationship between expression of the lymphangiogenic factor, VEGF-C, its receptors, VEGFR2 and VEGFR3, along with the canonical VEGF receptor VEGFR1 with disease-free (DFS) and OS (62-65). Although VEGF-C expression had a strong impact on DFS or OS in HER2+ tumors, HER2- patients with high VEGF-C and VEGFR1 expression had a worse prognosis overall, thus indicating a clinical relevance between VEGF-C expression and HER2 status (62).

With respect to ER status, we observed a significant negative effect on survival in ER+/HER2- individuals with high co-expression of VEGF and OSM or OSMR $\beta$. This is interesting in light of the fact that OSM has also been shown to negatively regulate expression of the estrogen receptor itself (66). Such regulation can indicate a key element in OSM-driven malignancy, with the possibility that ER+ tumor cells lose ER status over time and evolve to become less susceptible to hormone therapies and more difficult to treat (66). Furthermore, several in vitro studies have demonstrated that OSM has a greater effect inducing epithelial-mesenchymal transition (EMT), and tumor cell detachment effects in ER+ PR+ HER2- breast cancer cell lines such as T47D and MCF7 than on ERTNBCs like MDA-MB-231 or MDA-MB-468 $(15,30)$, and data not shown). Though our analysis for this work included 1,245 IDC patients, the assessment of HER2 and ER status yielded low population sizes suggesting that further examination may be needed. In particular, it is difficult to draw concrete conclusions between HER2+ status in relation to VEGF and inflammatory cytokines because of the low population sizes, even though clear statistical significance was shown with HER2- data. Overall, these results indicate that the inflammatory cytokines OSM and IL-6 contribute to the aggressive phenotype seen with HER2- disease. 
Standing alone, these results suggest important differences between breast cancer subtypes in relation to VEGF and IL6 family inflammatory cytokines. Based on our patient data demonstrating that high levels of IL-6 family cytokines and VEGF coexpression lead to poor survival in HER2- breast cancer, we investigated cytokineinduced VEGF secretion from two HER2- breast cancer cell lines, MDA-MB-231 and T47D. Typically, VEGF expression is regulated under both hypoxic and non-hypoxic conditions, as the promoter region contains several response elements that allow for VEGF regulation downstream of various signaling pathways (Fig. 6A, (42-45)). Our studies showed that OSM, IL-6, and LIF each increased expression of HIF1 $\alpha$, an important transcription factor for VEGF. However, OSM-induced VEGF was independent of HIF $1 \alpha$ in ER- MDA-MB-231 cells, whereas HIF $1 \alpha$ signaling was in part necessary for OSM-mediated VEGF induction in ER+ T47D cells. HIF1 $\alpha$ may have other alternate downstream effects in MDA-MB-231 cells such as the induction of angiopoietin-like-4, which has been shown to promote an aggressive phenotype (67). Furthermore, knockdown of STAT3 signaling ablated VEGF secretion in MDA-MB-231 cells while having less effect on VEGF secretion in T47D cells. This difference demonstrates that TNBC MDA-MB-231 cells depend on activation of STAT3 for induction of VEGF secretion and suggests that inhibition of cytokine stimulation may attenuate angiogenesis in TNBC disease. To summarize, OSM-induced VEGF secretion is dependent on the activation of STAT3 in MDA-MB-231 cells, while activation of both STAT3 and HIF $1 \alpha$ appear necessary for VEGF induction in T47D cells (Fig. 6B). These results may indicate unique properties pertaining to angiogenic signaling in ER- TNBC versus ER+ PR+ HER2- tumors. 
Though it might be expected that IL-6 should also induce VEGF secretion from breast cancer cells, we found that in ER- MDA-MB-231 cells, IL-6 did not activate the STAT3 pathway and therefore did not promote VEGF secretion. Previous studies have shown that IL-6 has no effect on MDA-MB-231 E-cadherin levels (known to be signaled through the JAK/STAT3 pathway) and on various other markers of invasion (68), which suggests that while ER- MDA-MB-231 cells express the IL-6 receptor, STAT3 signaling is not regulated by IL-6 in these cells. While we showed that IL-6 weakly activated the STAT3 pathway in ER+ T47D cells, this induction was only seen through the first hour of treatment and did not extend to 48 hours as did OSM-induced pSTAT3. Interestingly, under innate immunological stress such as mycoplasma contamination, overall STAT3 activation was enhanced in T47D cells and pSTAT3 induction by IL-6 mirrored that of OSM (data not shown). Mycoplasma-induced pSTAT3 has been previously seen in airway epithelial lung cells and is thought to be part of the cause for inflammation during mycoplasma infections (69). Overall, the activation of STAT3 signaling by OSM is significantly greater than with IL-6 and highlights a difference in signaling magnitude and possibly function between these two cytokines in a breast cancer subtype specific manner.

\section{Conclusions}

Taken together, our studies demonstrate that co-expression of VEGF and IL-6 family cytokine molecules emerge as a potential negative prognostic marker, particularly for HER2- IDC disease. Furthermore, we demonstrate that there is distinct differential cytokine-induced signaling of STAT3 and HIF1 $\alpha$, which lead to varied levels of VEGF secretion among various TNBC and ER+ PR+ HER2- breast tumor cells. Collectively, 
these results suggest that IL-6 family cytokine inhibition may have a beneficial effect on VEGF suppression and long-term patient survival in HER2- disease. 


\section{CHAPTER TWO: FIGURES}

A

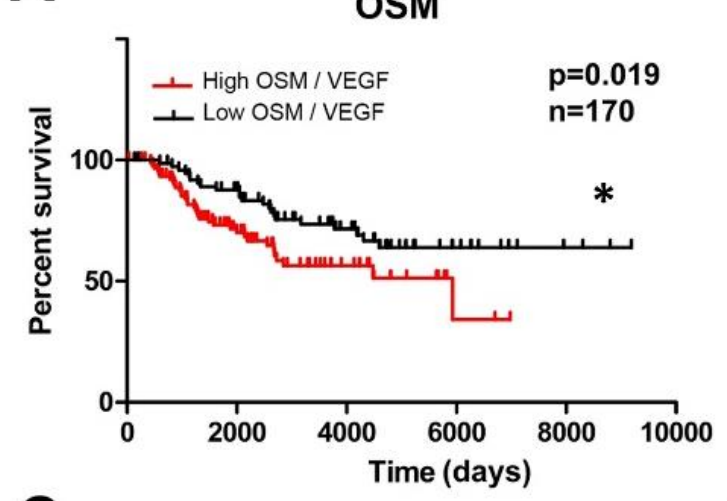

C

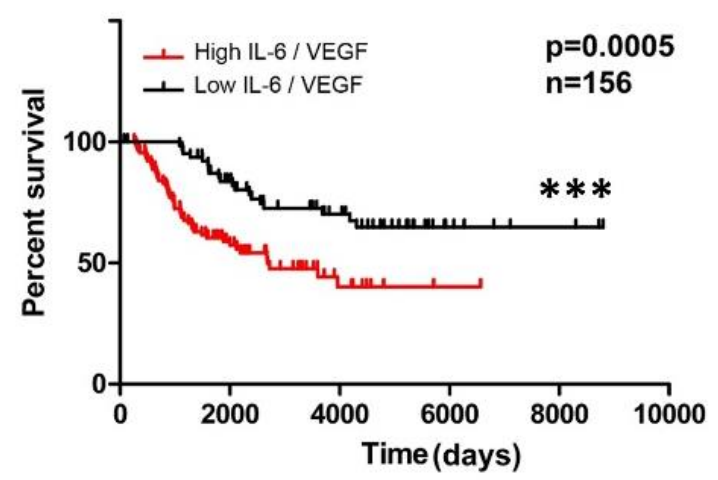

$\mathbf{E}$

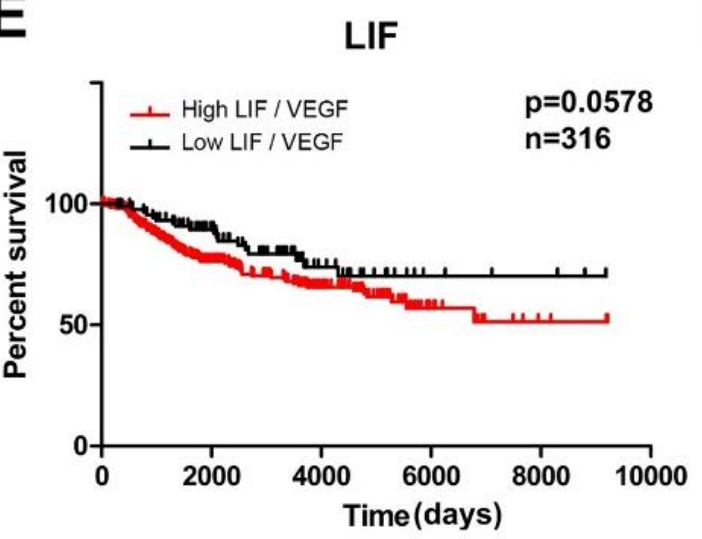

B

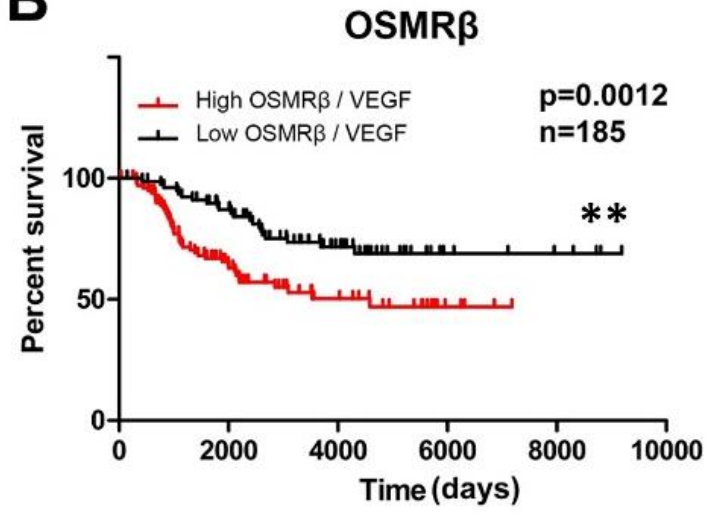

D

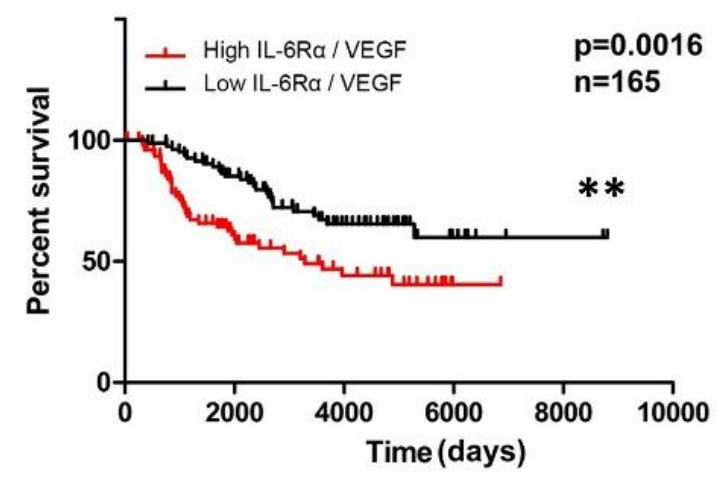

F

LIFR $\beta$

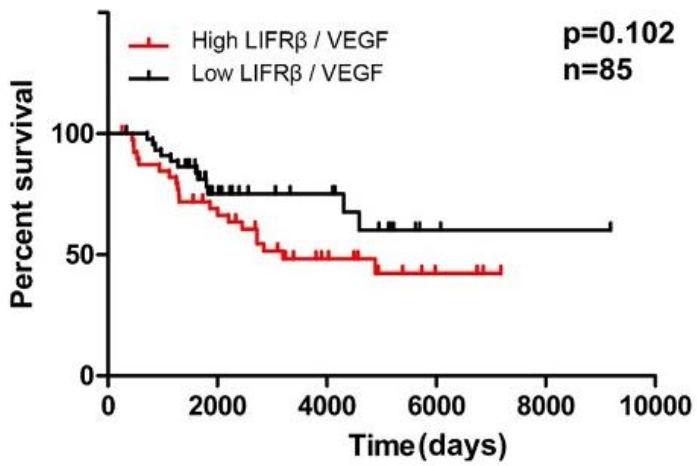

Figure 2.1. Increased co-expression of OSM or OSMR 3 and VEGF correlates with decreased survival of IDC patients 
Figure 2.1. Inflammatory cytokine and VEGF co-expression are correlated with decreased survival of invasive ductal carcinoma patient.

These Kaplan-Meier curves in Figure 1A and B are a subset of the data presented in Supplemental Figure S1. A) Kaplan-Meier survival curves of invasive ductal breast carcinoma patients with high OSM and VEGF expression (upper quartiles, respectively) present diminished survival when compared to individuals with low OSM and VEGF expression (lower quartiles, respectively). Comparable trends appear upon examination of the upper and lower quartiles of patients with B) OSMR $\beta$ and VEGF expression, C) IL-6 and VEGF expression, and D) IL-6R $\alpha$ and VEGF expression, indicating significant differences between upper and lower quartile survival for each group. Survival of patients with invasive ductal breast carcinoma by E) LIF and VEGF expression and F) LIFR $\beta$ and VEGF expression are not significantly different. Analysis obtained from Oncomine ${ }^{\mathrm{TM}}$ dataset entitled Curtis Breast. Log-rank test $* \mathrm{p}<0.05, * * \mathrm{p}<0.01, * * * \mathrm{p}<0.001$. 

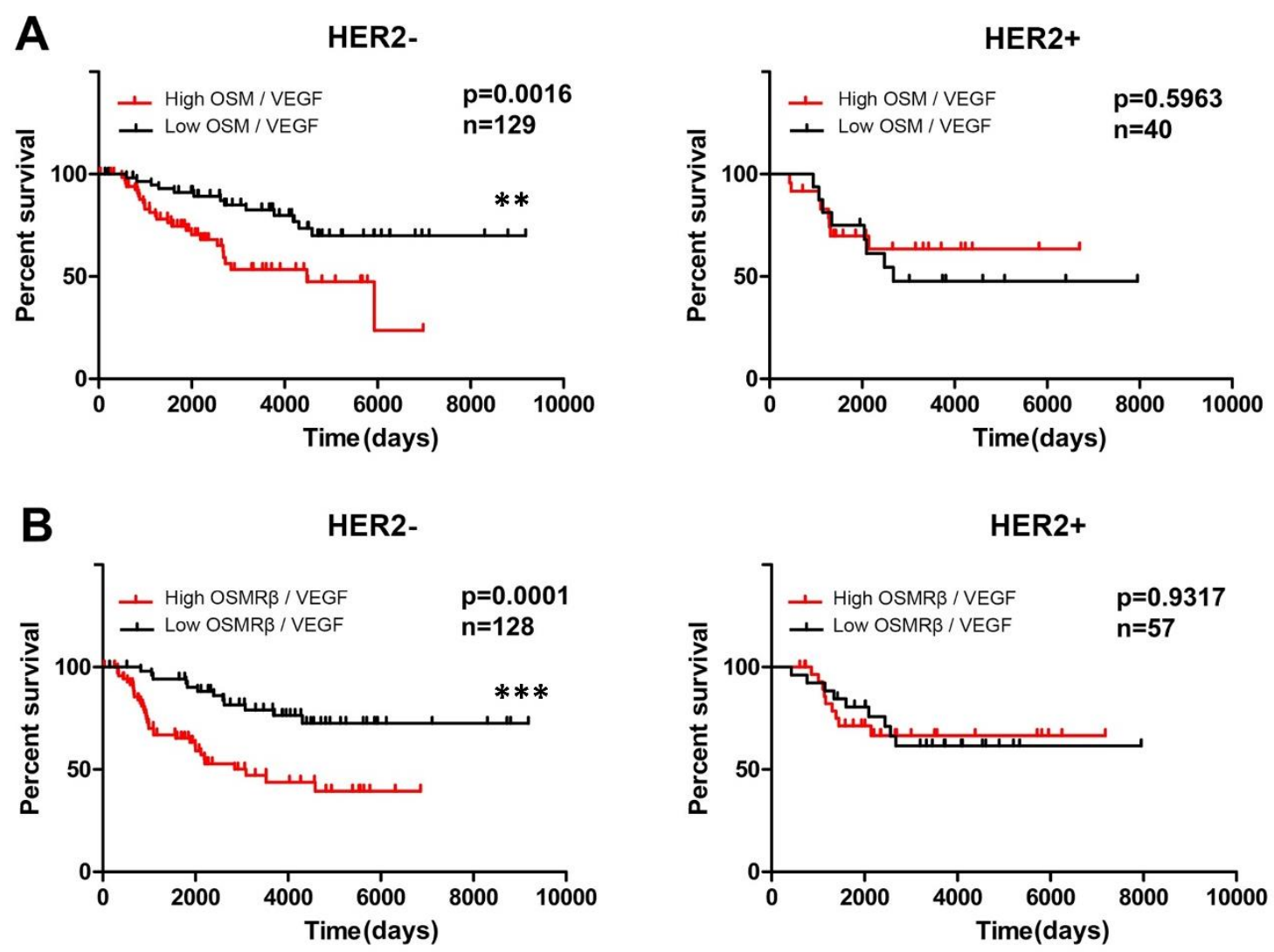

Figure 2.2. Co-expression of VEGF with OSM or OSMRß appears to affect survival in HER2- but not HER2+ subtypes 
Figure 2.2 Co-expression of VEGF with OSM or OSMR $\beta$ appears to affect survival in HER2- but not HER2+ subtypes.

A) Kaplan-Meier survival curves of invasive ductal breast carcinoma for HER2patients and HER2+ patients by OSM and VEGF expression. In HER2- patients, high OSM and high VEGF expression (upper quartiles, respectively) is strongly correlated with decreased survival when compared to patients with low OSM and low VEGF expression (lower quartiles, respectively). However, this trend is not evident in HER2+ individuals, as no significant difference is observed between survival of patients with OSM and VEGF expression in upper and lower quartiles, respectively. Similar trends are observed in Kaplan-Meier survival curves for B) HER2- and HER2+ by OSMR $\beta$ and VEGF expression. Analysis obtained from Oncomine ${ }^{\mathrm{TM}}$ dataset entitled Curtis Breast. Log-rank test $* \mathrm{p}<0.05, * * \mathrm{p}<0.01, * * * \mathrm{p}<0.001$ 
A

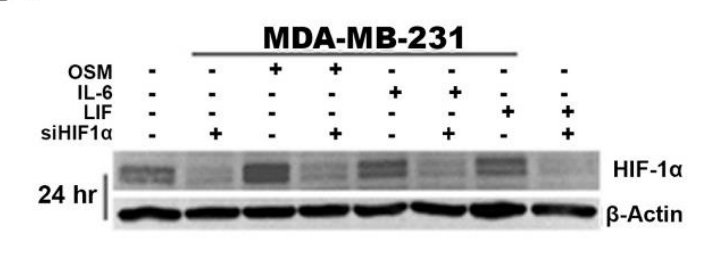

C
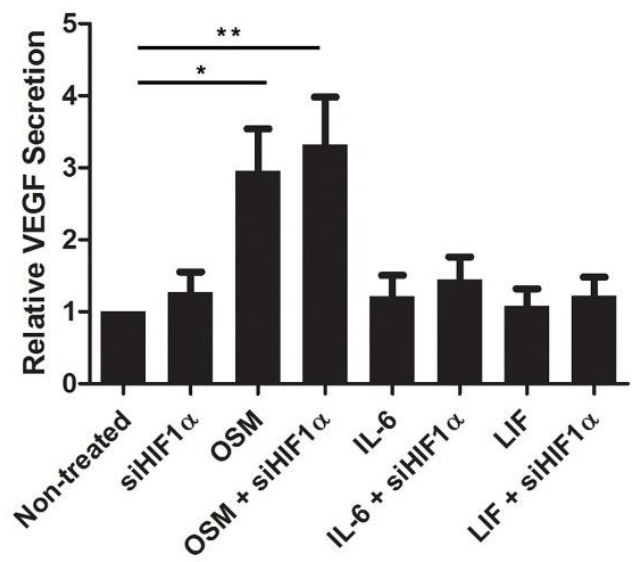

B

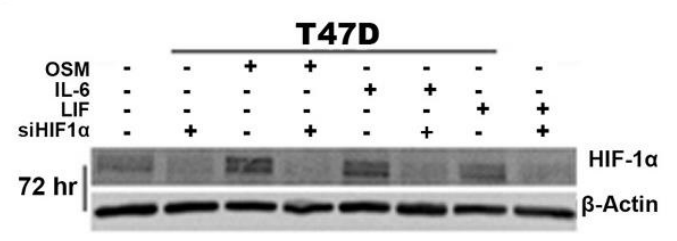

D

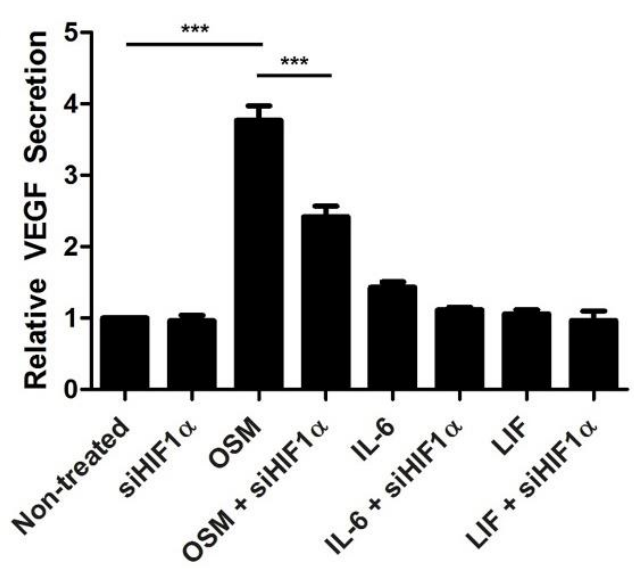

Figure 2.3. IL-6 family cytokine OSM induces VEGF secretion independent of HIF1 $\alpha$ signaling in MDA-MB-231 cells

Figure 2.3. IL-6 family cytokine OSM induces VEGF secretion independent of HIF1 $\alpha$ signaling in MDA-MB-231 cells.

A) Treatment with OSM, IL-6, and LIF ( $25 \mathrm{ng} / \mathrm{mL})$ for 24 hours promotes expression of HIF1 $\alpha$ in MDA-MB-231 TNBC cells. Addition of a siRNA targeting HIF1 $\alpha$ (siHIF1 $\alpha$ ) ablates the effect of adding cytokines. B) T47D cells treated with IL-6 family cytokines for 72 hours express greater levels of HIF1 $\alpha$ relative to non-treated control. Treatment with siHIF1 $\alpha$ reduces this effect, as assessed by immunoblot analysis. Immunoblots are representative of at least 3 experiments. Induction of VEGF secretion is observed following treatment with IL-6 family cytokines $(25 \mathrm{ng} / \mathrm{mL})$ for 24 hours in C) MDA-MB-231 cells ( $\mathrm{n}=5$ ) and for 72 hours in $\mathbf{D})$ T47D cells ( $\mathrm{n}=3$ ), as determined by ELISA. Treatment with siHIF $1 \alpha$ does not affect VEGF secretion in OSM-treated MDAMB-231 cells; however, VEGF secretion is modestly reduced in T47D cells treated with 
OSM and siHIF1 $\alpha$. Data expressed as mean +/- SEM. One-way ANOVA, Tukey's posttest, ${ }^{*} * \mathrm{p}<0.01, * * * \mathrm{p}<0.001$.

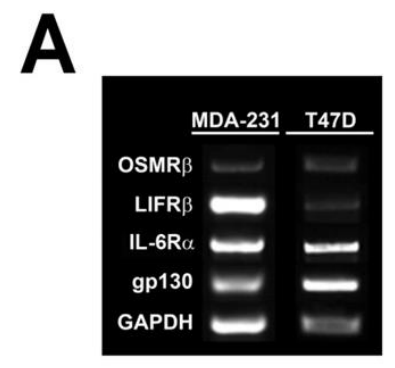

B
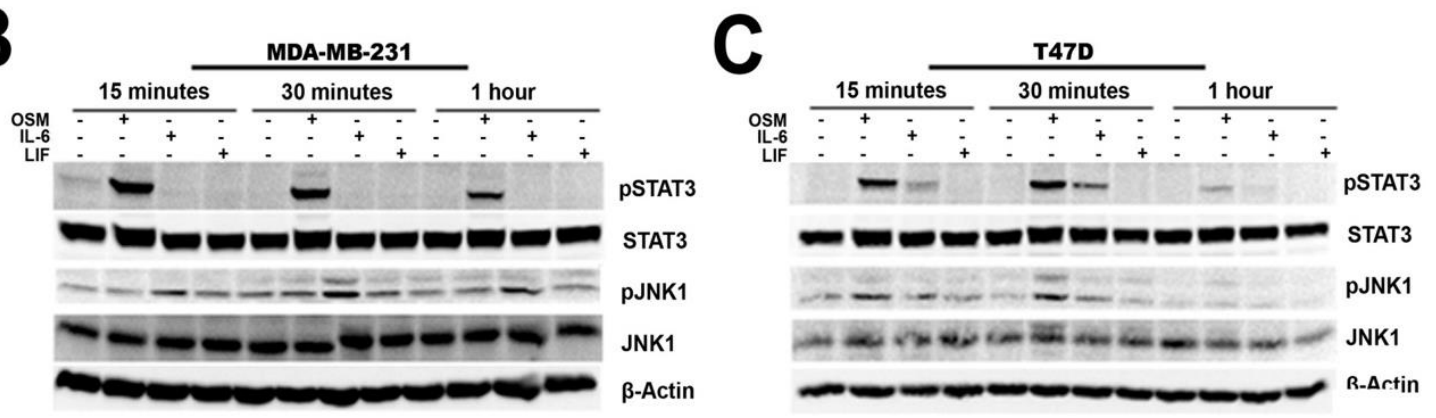

D
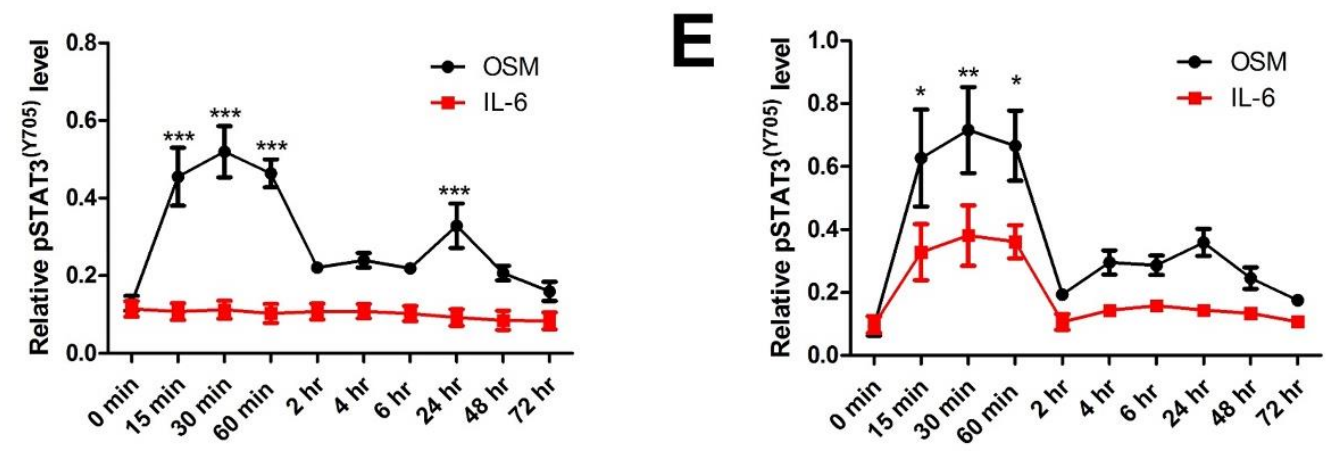

Figure 2.4. OSM strongly induces phosphorylation of STAT3 
Figure 2.4. OSM strongly induces phosphorylation of STAT3.

A) mRNA expression by RT-PCR of OSMR $\beta$, LIFR $\beta$, IL-6R $\alpha$, and gp130 in MDA-MB-231 and T47D cells. B) Treatment with OSM (25 ng/mL) for 15 minutes, 30 minutes, and 1 hour strongly induces phosphorylation of STAT3 (Tyr 705) in MDA-MB231 cells and moderately induces JNK phosphorylation (T183/Y185). Treatment with either IL-6 or LIF (25 ng/mL) does not induce pSTAT3 or pJNK. C) Treatment with OSM induces phosphorylation of STAT3 and JNK in T47D cells. IL-6 induces moderate phosphorylation of STAT3 and JNK at early time points. Phosphorylation of STAT3 and JNK was compared to control $\beta$-actin, as assessed by immunoblot analysis. Blots are representative of three experiments. Time course experiment for STAT3 phosphorylation upon treatment with OSM or IL-6 (25 ng/mL) for 0.25-72 hours in D) MDA-MB-231 cells, and in E) T47D cells. Treatment with IL-6 does not induce pSTAT3 expression in MDA-MB-231 cells, while inducing moderate pSTAT3 expression in T47D cells. ELISA was performed in quadruplicate; with each data point expressed as mean +/- SEM. Twoway ANOVA with Bonferroni post-test, $* \mathrm{p}<0.05, * * \mathrm{p}<0.01, * * * \mathrm{p}<0.001$. 

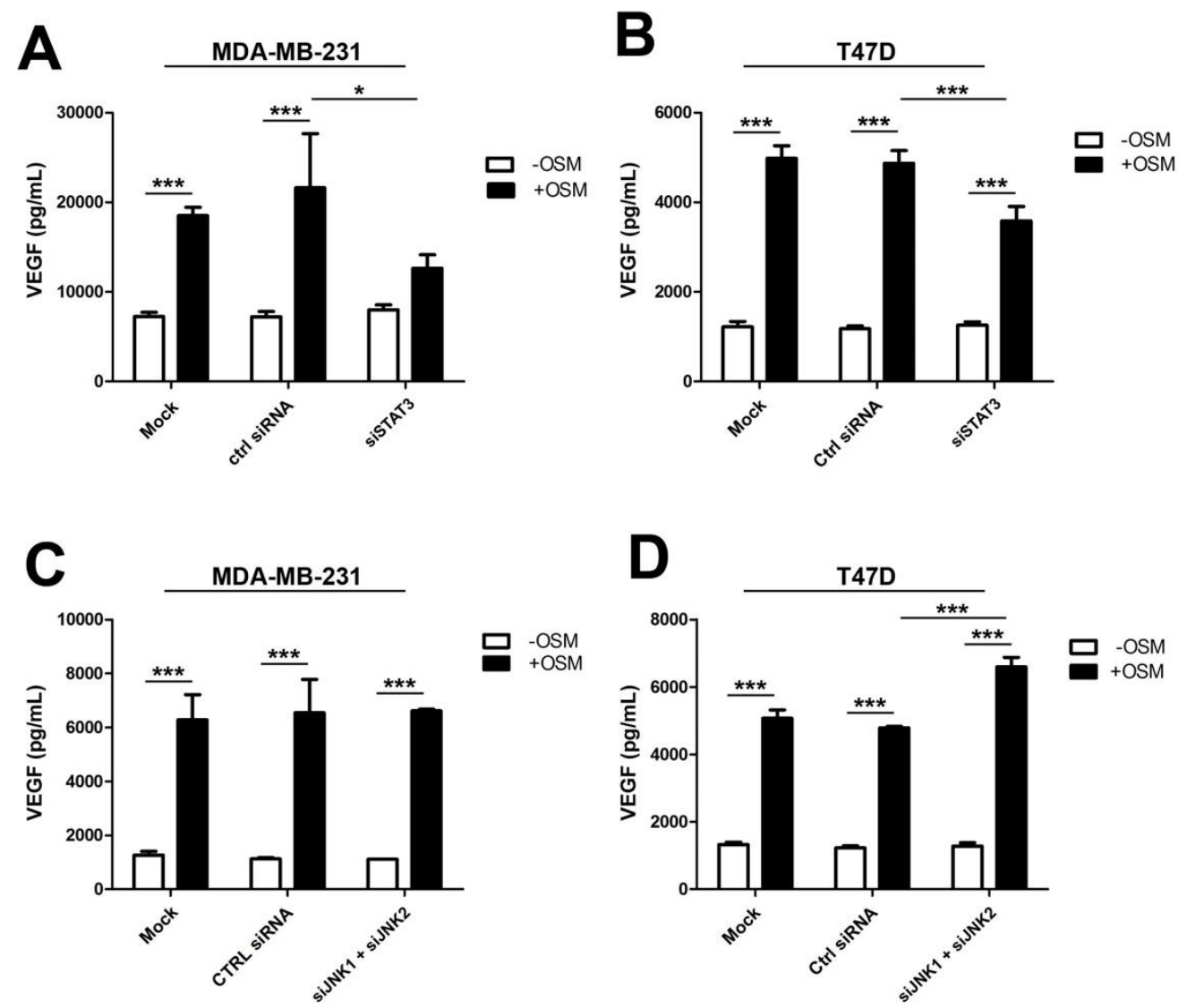

Figure 2.5. OSM induces VEGF via STAT3 signaling

Figure 2.5. OSM induces VEGF via STAT3 signaling.

A) Treatment with OSM ( $25 \mathrm{ng} / \mathrm{mL})$ and siSTAT3 suppresses VEGF secretion by 3-fold in MDA-MB-231 cells. B) Treatment with OSM and siSTAT3 moderately reduces VEGF secretion by T47D cells. VEGF secretion was not reduced by the addition of siRNAs targeting both JNK1 and JNK2 in either C) MDA-MB-231 or D) T47D cells. Experiments were performed in triplicate, and data expressed as mean +/- SEM. Twoway ANOVA with Bonferroni post-test, ${ }^{*} \mathrm{p}<0.05,{ }^{* * *} \mathrm{p}<0.001$. 


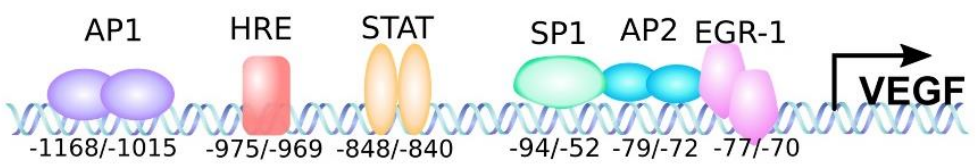

B

MDA-MB-231 (Triple Negative)

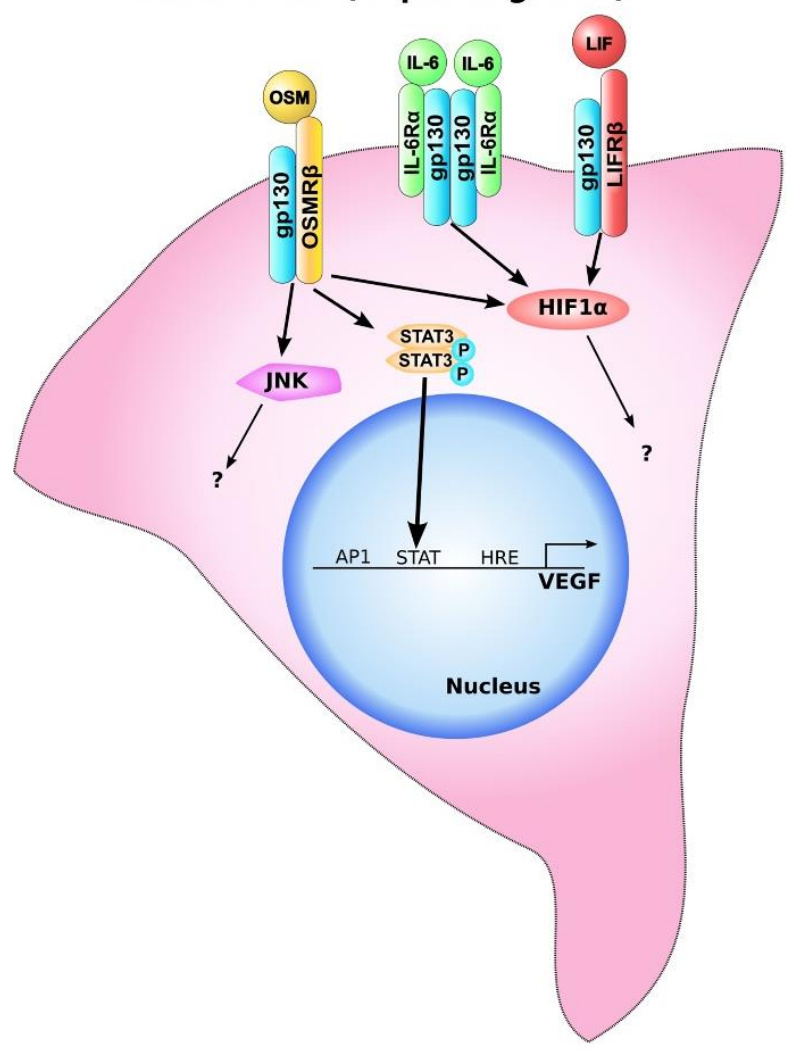

T47D (ER/PR+ HER2-)

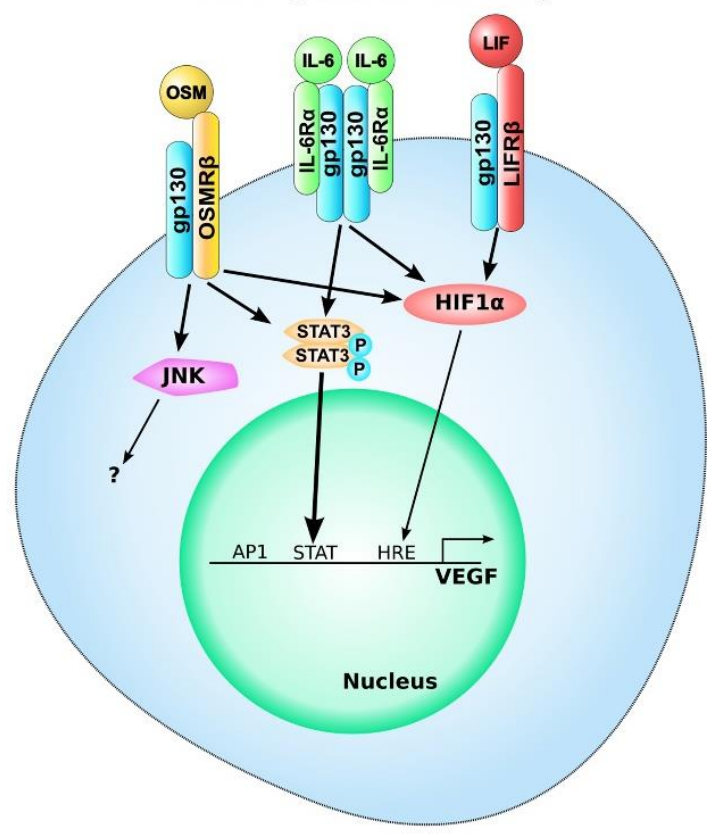

Figure 2.6. Mechanistically distinct regulation of VEGF secretion in MDA-MB231 TNBC and T47D (ER+/PR+/HER2-) cells

Figure 2.6. Mechanistically distinct regulation of VEGF secretion in MDA-MB-231

TNBC and T47D (ER+/PR+/HER2-) cells.

A) The VEGF promoter can be activated by various transcription factors. B) In MDA-MB-231 cells, OSM mediates VEGF secretion by activating the STAT3 signaling pathway downstream of the OSMR (OSMR $\beta+$ gp 130). In T47D cells, OSM regulates VEGF secretion via both HIF1 $\alpha$ and the STAT3 signaling pathway. 


\section{Supplemental Materials and Methods}

Endothelial Cell Tube and Branch Point Formation Assay

For the in vitro Angiogenesis Assay (Chemicon), Matrigel $(50 \mu \mathrm{L})$ was allowed to solidify in 96 -well plates at $37^{\circ} \mathrm{C}$ for 90 minutes prior to the addition of HUVECs $(7.5 \mathrm{x}$ $10^{3}$ cells). Cells were allowed to adhere for one hour in serum-free M199 medium. Medium was then removed and the HUVECs were treated with 1) conditioned media (CM) from untreated MDA-MB-231 cells, 2) CM from OSM-treated MDA-MB-231 cells, 3) control medium with $25 \mathrm{ng} / \mathrm{mL}$ rhOSM, or 4) control medium with $50 \mathrm{ng} / \mathrm{mL}$ rhVEGF. Additionally, HUVECs were treated with OSM in combination with either 0.6 $\mu \mathrm{g} / \mathrm{mL}$ anti-OSM neutralizing antibody or $0.6 \mu \mathrm{g} / \mathrm{mL}$ anti-VEGF neutralizing antibody (Genentech). Tube formation was imaged every hour for an eight-hour period using $600 \mathrm{x}$ 800 pixel digital images. To quantify tube formation, branch points were counted as angle formed by an extending cellular process.

$\underline{\text { In vivo Matrigel Plug Assay }}$

The Matrigel plug assay was performed as described previously (70) with the following modifications. Briefly, 8-week old female athymic NCr-nu/nu nude mice were injected subcutaneously and bilaterally in dorsal groin area with $0.3 \mathrm{~mL}$ of Matrigel (BD Biosciences) containing 20 units heparin and one of the following: 1) PBS, 2) $200 \mathrm{ng}$ VEGF in PBS, 3) control MDA-MB-231 CM (concentrated 3x), 4) OSM-treated MDAMB-231 conditioned medium (concentrated 3x), 5) CM plus $0.6 \mu \mathrm{g} / \mathrm{mL}$ anti-OSM neutralizing antibody or 6) CM plus anti-VEGF neutralizing antibody. After 10 days, the Matrigel plugs were removed, trimmed of mouse tissue, photographed, and weighed. Hemoglobin was measured using the Drabkin method (Sigma-Aldrich) following 
homogenization in $0.5 \mathrm{~mL}$ distilled water. Concentrations of hemoglobin were calculated from known hemoglobin concentrations based on a standard curve run in parallel.

Athymic NCR-nu/nu mice were housed in environmentally controlled rooms in the

Veteran's Administration Medical Center animal facility in Boise, ID. All procedures were approved by the International Animal Care and Use Committee.

JNK Inhibitor Assay

MDA-MB-231 cells were grown to 70-80\% confluency on a 24-well plate with RPMI-1640 media. Cells were treated with 100 nM JNK Inhibitor II (CAS 129-56-6, Cal Biochem). After four hours, $25 \mathrm{ng} / \mathrm{mL}$ OSM was added for 30 minutes, after which cell lysates were collected and examined by immunoblot analysis.

\section{Chapter Two: Supplemental Figures}
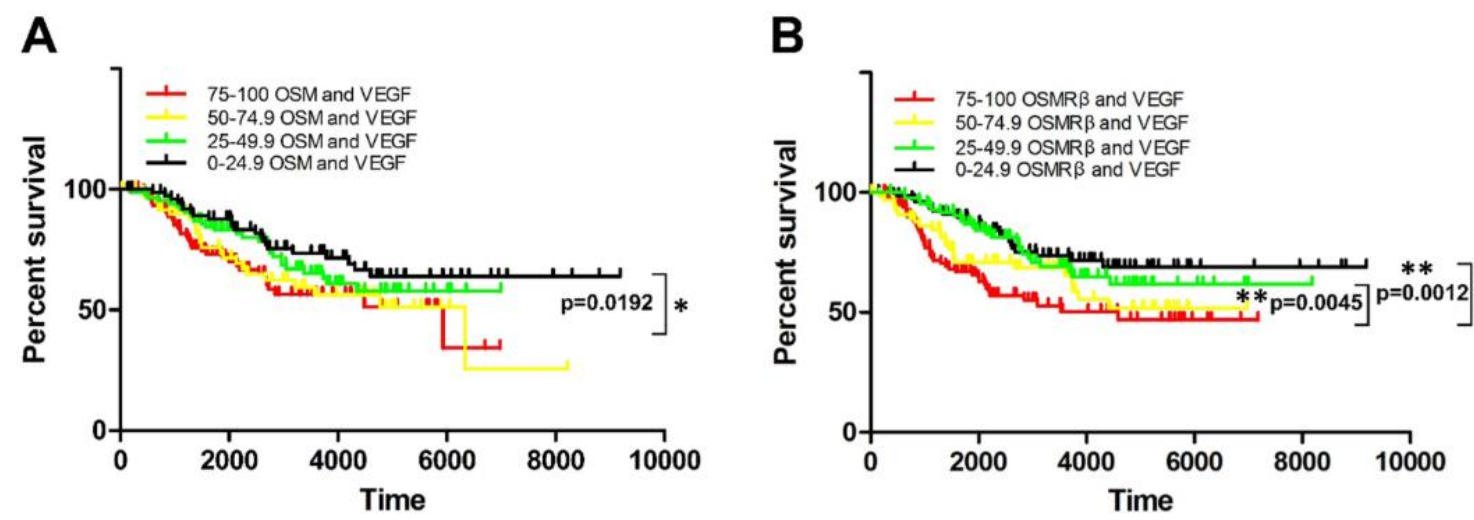

Figure 2.S1. Mechanistically distinct regulation of VEGF secretion in MDA-MB231 TNBC and T47D (ER+/PR+/HER2-) cells 
Figure 2.S1. Increased co-expression of OSM or OSMR $\beta$ and VEGF correlates with decreased survival of IDC patients.

Kaplan-Meier survival curves of invasive ductal breast carcinoma for A) OSM and VEGF ( $\mathrm{n}=318)$ and B) OSMR $\beta$ and VEGF expression $(\mathrm{n}=389)$, as separated by quartiles. As co-expression of OSM and VEGF increases, overall survival trends decrease, with patients expressing high OSM high VEGF (upper quartiles, respectively) exhibiting significantly worse survival $(\mathrm{p}=0.0192)$ than individuals expressing low OSM low VEGF (lower quartiles, respectively). A comparable trend is observed with coexpression of OSMR $\beta$ and VEGF ( $p=0.0012)$. Analysis obtained from Oncomine ${ }^{\mathrm{TM}}$ dataset entitled Curtis Breast. Log-rank test $* \mathrm{p}<0.05, * * \mathrm{p}<0.01$. 
A

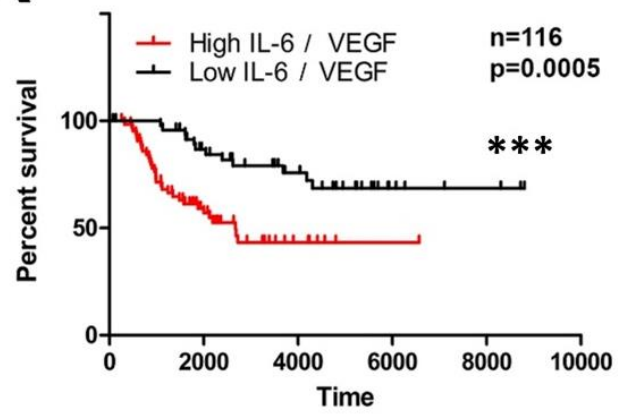

B

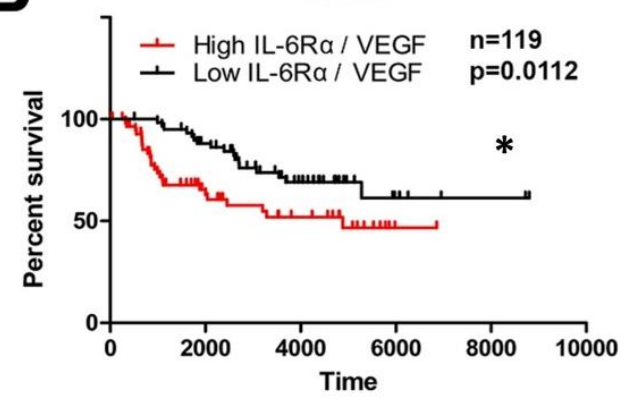

C

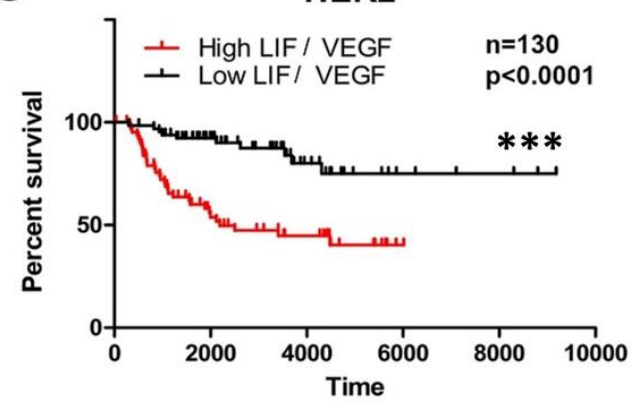

D

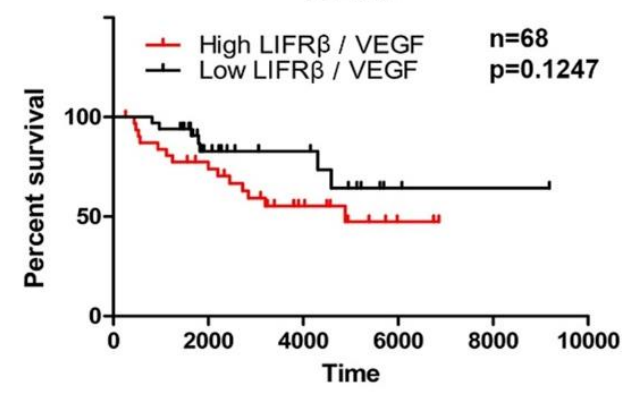

HER2+

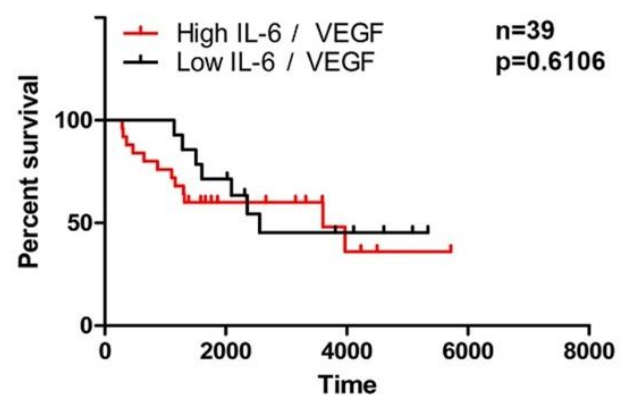

HER2+

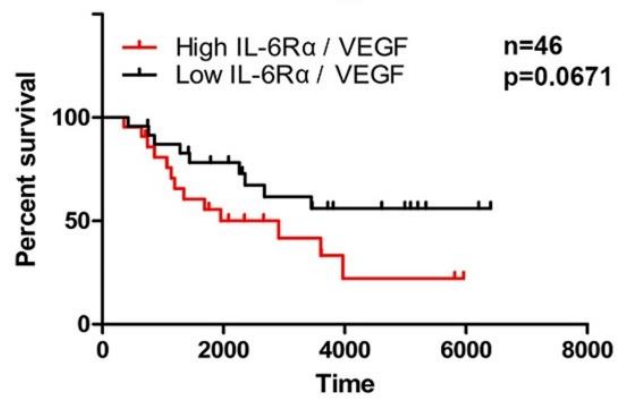

HER2+

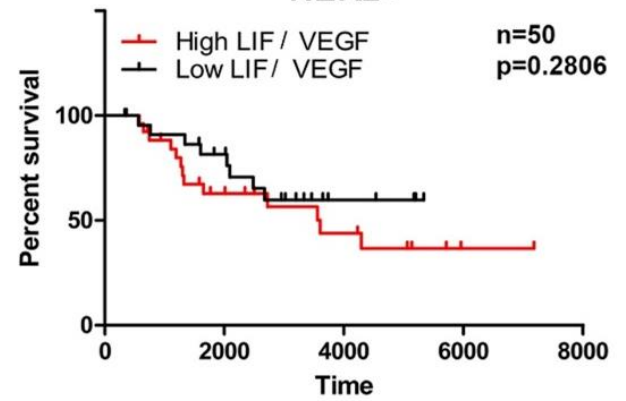

HER2+

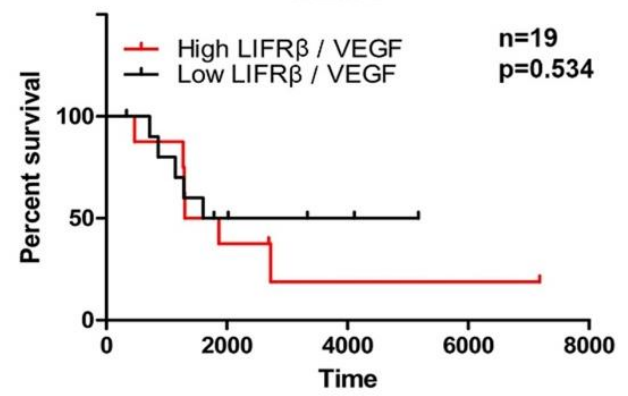

Figure 2.S2. Expression of cytokines or their receptors and VEGF appears to affect survival in HER2- but not HER2+ subtypes 
Figure 2.S2. Expression of cytokines or their receptors and VEGF appears to affect survival in HER2- but not HER2+ subtypes.

Kaplan-Meier survival curves of invasive ductal breast carcinoma for A) HER2and HER2+ patients by IL-6 and VEGF expression. In HER2- patients, high IL-6 and high VEGF expression (upper quartiles, respectively) is strongly correlated with decreased survival when compared to patients with low IL-6 and low VEGF expression (lower quartiles, respectively). However, this trend is not evident in HER2+ individuals. Similar trends are observed in Kaplan-Meier survival curves between HER2- and HER2+ patients by B) IL-6R $\alpha$ and VEGF expression and C) LIF and VEGF expression. D) Coexpression of LIFR $\beta$ and VEGF does not significantly affect survival in either HER2- or HER2+ patients. Analysis obtained from Oncomine ${ }^{\mathrm{TM}}$ dataset entitled Curtis Breast. Logrank test $* \mathrm{p}<0.05, * * \mathrm{p}<0.01, * * * \mathrm{p}<0.001$ 
A

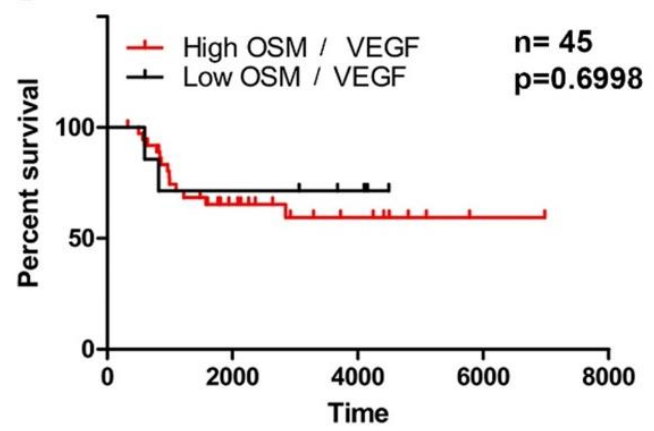

B

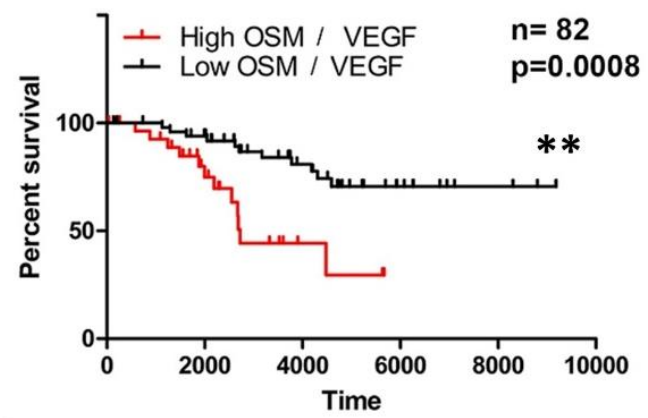

C

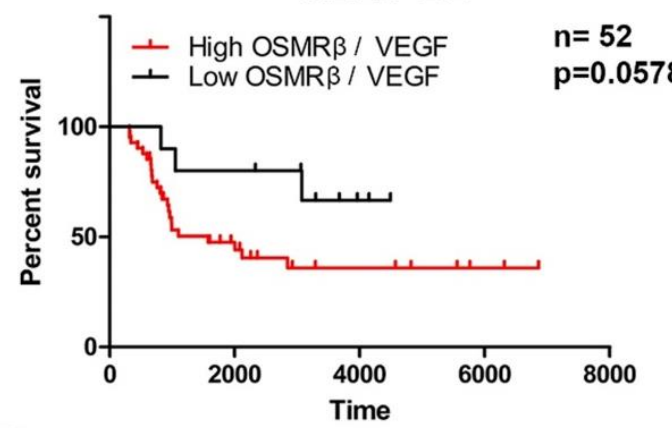

D

HER2- ER+

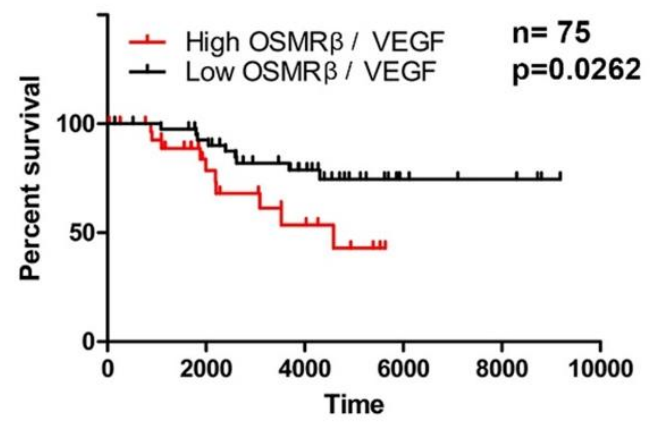

HER2+ ER-

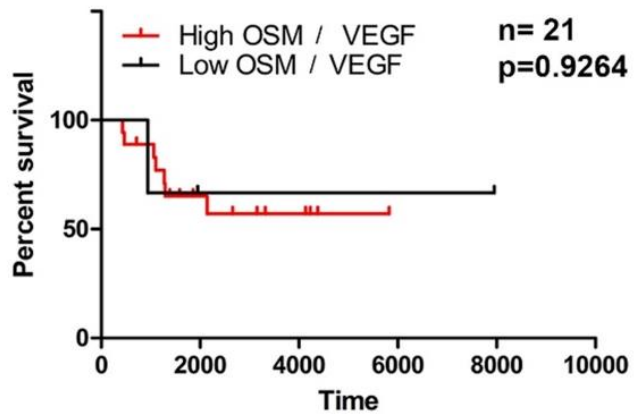

HER2+ ER+

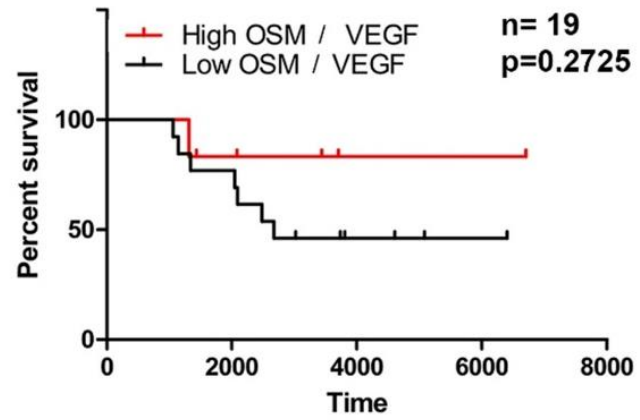

HER2+ ER-

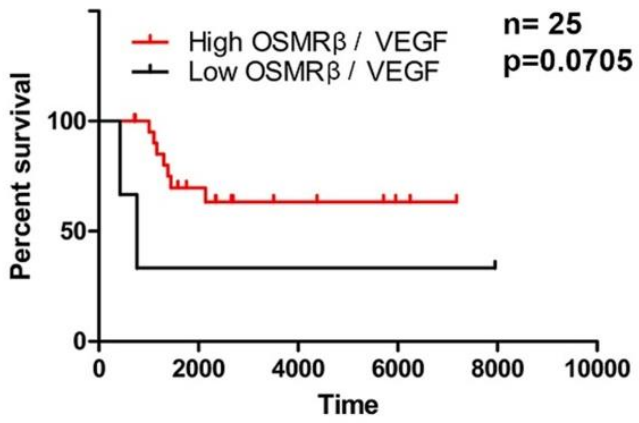

HER2+ ER+

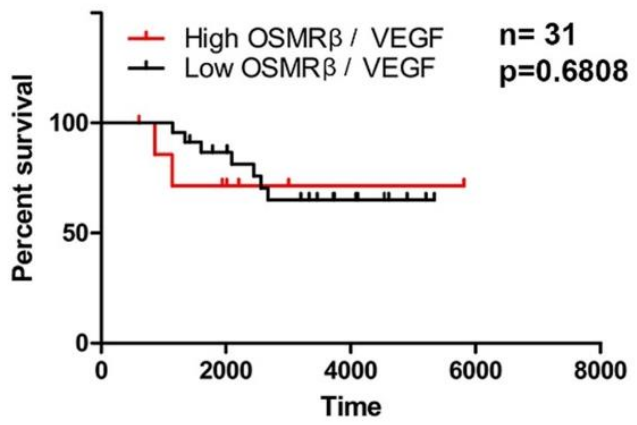

Figure 2.S3. Co-expression of OSM or OSMR $\beta$ and VEGF differentially affects survival based on estrogen receptor status 
Figure 2.S3. Co-expression of OSM or OSMR $\beta$ and VEGF differentially affects survival $\underline{\text { based on estrogen receptor status. }}$

Kaplan-Meier survival curves of invasive ductal breast carcinoma for A) HER2/ER- and HER2+/ER- patients and B) HER2-/ER+ and HER2+/ER+ patients by OSM and VEGF expression. In patients with either HER2+/- and ER- expression, OSM/VEGF expression levels do not significantly affect survival. However, in HER2-/ER+ patients, high OSM and high VEGF expression (upper quartiles, respectively) is strongly correlated with decreased survival when compared to patients with similar receptor status expressing low OSM and low VEGF expression (lower quartiles, respectively). Similar trends respective of receptor status are observed in Kaplan-Meier survival curves for $\mathbf{C}$ ) HER2-/ER- and HER2+/ER- patients and D) HER2-/ER+ and HER2+/ER+ patients by OSMR $\beta$ and VEGF expression. While no correlation between OSM/VEGF expression and survival is observed in HER2+/ER+ patients, the low number of samples in these populations distorts our ability to make a robust assessment of this patient subset. Analysis obtained from Oncomine ${ }^{\mathrm{TM}}$ dataset entitled Curtis Breast. Log-rank test $* \mathrm{p}<0.05, * * \mathrm{p}<0.01$ 

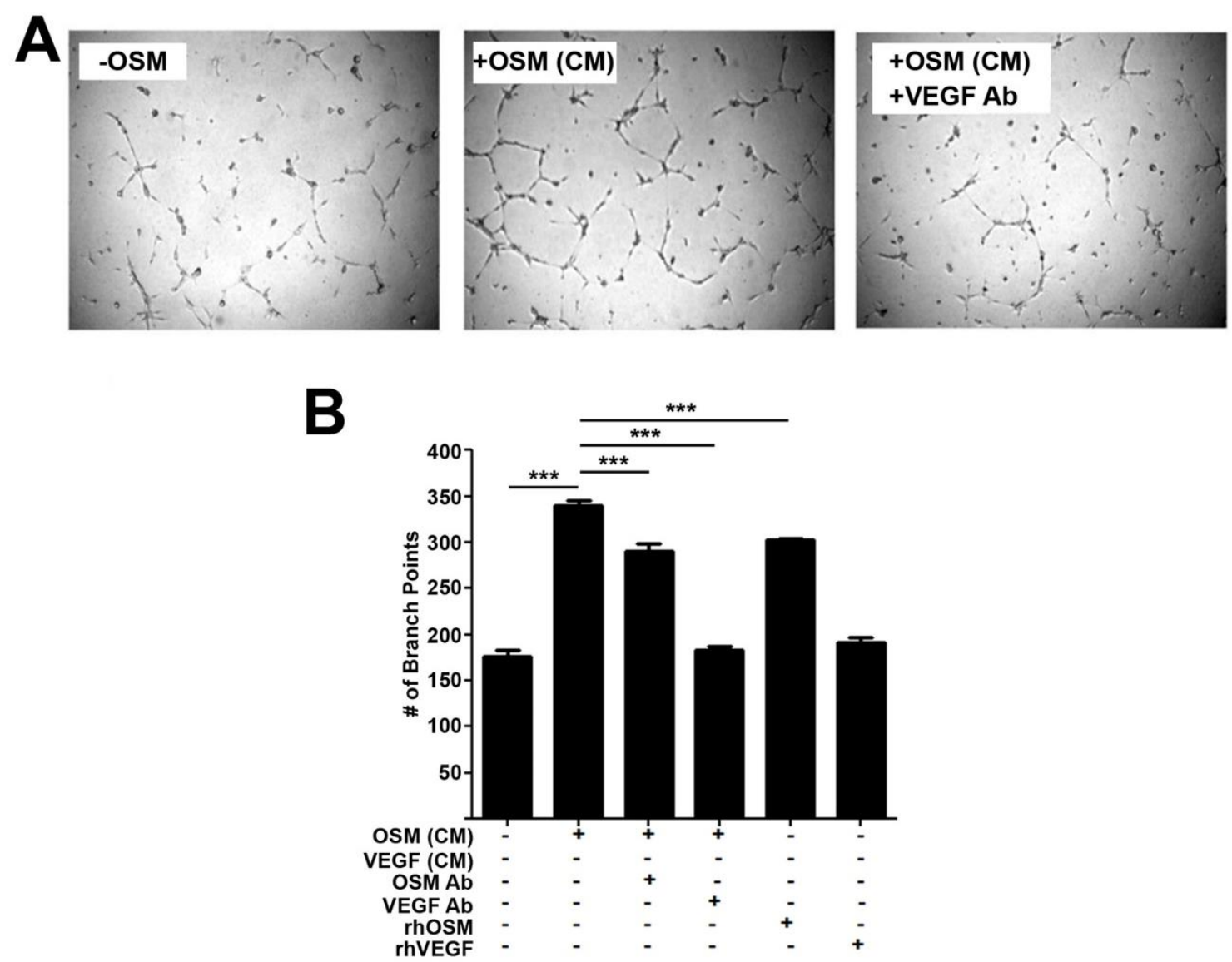

Figure 2.S4. OSM induces endothelial cell tube formation and branch points in vitro 
Figure 2.S4. OSM induces endothelial cell tube formation and branch points in vitro.

Tube formation of human umbilical vein endothelial cells (HUVEC) cells treated for 4 hours with conditioned media from A) MDA-MB-231 treated with OSM (25 $\mathrm{ng} / \mathrm{mL})$ with or without anti-VEGF neutralizing antibody $(0.6 \mu \mathrm{g} / \mathrm{mL})$; light microscopy, 200X. Increased tube formation is observed in HUVECs incubated with conditioned media (CM) of OSM-treated breast cancer cells, as compared to HUVECs incubated with control CM. B) Quantification of branch points was obtained by counting the number of tube-like protrusions originating from each cell node. A two-fold increase in branch points is observed in HUVECs treated with CM of OSM-treated MDA-MB-231 cells compared to non-treated control CM. The addition of anti-VEGF neutralizing antibody reduces branch point numbers to control levels, while anti-OSM neutralizing antibody shows a partial effect. Addition of recombinant VEGF or OSM to untreated cell CM are included as controls. Data expressed as mean +/- SEM. One-way ANOVA with Tukey's post-test, $* * * \mathrm{p}<0.001, \mathrm{n}=5$. 

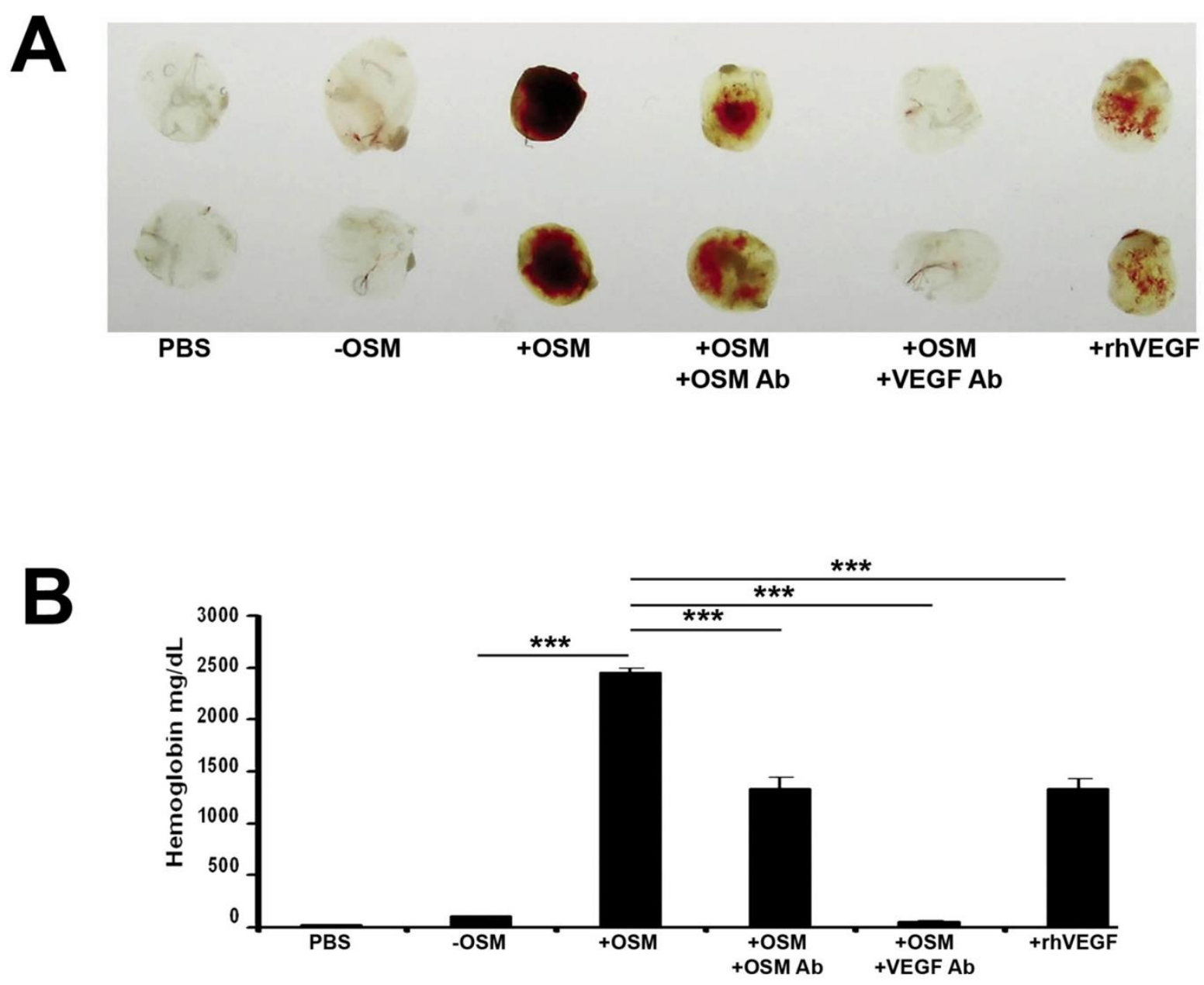

Figure 2.S5. OSM-treatment of human breast cancer cells stimulates angiogenesis in vivo 
Figure 2.S5. OSM-treatment of human breast cancer cells stimulates angiogenesis in vivo.

Six- to eight-week old female athymic nude mice were injected with $0.3 \mathrm{~mL}$ of Matrigel containing heparin (20 units) and $100 \mu \mathrm{L}$ of one of the following: 1) PBS; 2) untreated MDA-MB-231 cell conditioned medium, concentrated 3-fold (3X); 3) OSMtreated (25 ng/mL) MDA-MB-231 cell conditioned medium (3X); 4) OSM-treated medium $(3 X)+$ anti-OSM neutralizing antibody $(0.6 \mu \mathrm{g} / \mathrm{mL}) ; 5)$ OSM-treated medium $(3 \mathrm{X})+$ anti-VEGF neutralizing antibody $(0.6 \mu \mathrm{g} / \mathrm{mL})$; or 6$)$ untreated control cell medium + rhVEGF $(50 \mathrm{ng} / \mathrm{mL})$. After 10 days the mice were euthanized and the Matrigel plugs were removed. A) Representative plugs containing various treatments were photographed. B) Quantification of new infiltrating vessel formation was determined by the measurement of the amount of hemoglobin in each Matrigel plug using the Drabkin's assay. Hemoglobin levels increases 12-fold for OSM-treated conditioned medium over untreated conditioned medium. Data was analyzed from at least 6 replicates per condition. Data expressed as mean +/- SEM. One-way ANOVA with Tukey's post-test, $* * * p<0.001$. 

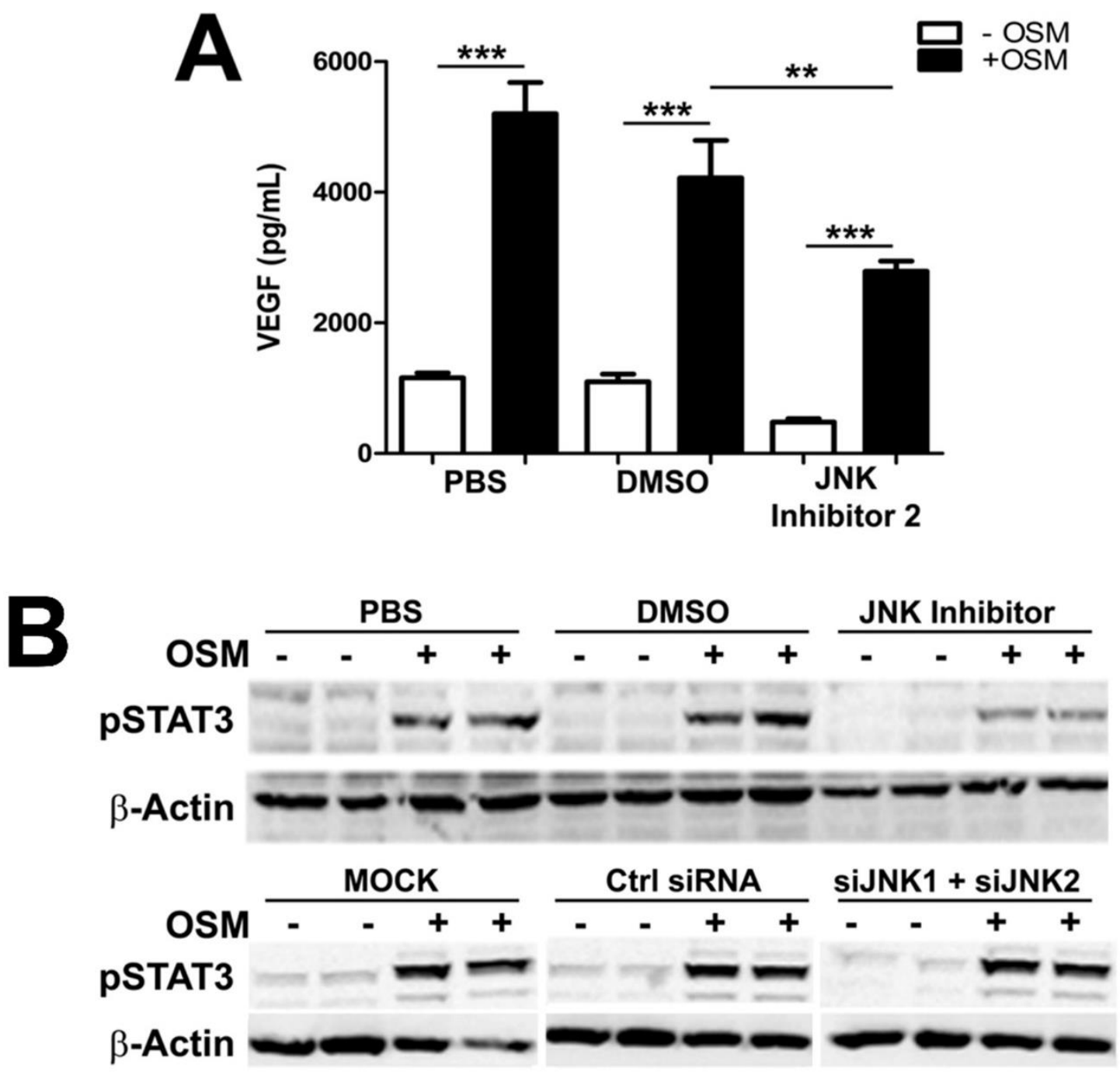

Figure 2.S6. JNK Inhibitor 2 suppresses VEGF secretion and phosphorylation of STAT3 
Figure 2.S6. JNK Inhibitor 2 suppresses VEGF secretion and phosphorylation of STAT3.

A) MDA-MB-231 cells were treated with JNK Inhibitor 2 or control DMSO for 2 hours prior to treatment with OSM (25 ng/mL). VEGF levels were assessed following treatment with OSM for 48 hours by ELISA. JNK Inhibitor 2 suppresses overall VEGF levels by roughly $50 \%$. JNK ELISA was performed in triplicate. B) Phosphorylation of STAT3 is suppressed in cell lysates collected from cells treated with JNK Inhibitor 2 and OSM, as assessed by immunoblot analysis, indicating non-specific effects of the JNK Inhibitor. For the VEGF ELISA, the data was expressed as mean +/- SEM, and assessed by two-way ANOVA with Bonferroni post-test was run; **p<0.01, ***p<0.001. 


\section{References}

1. Society AC. Cancer Facts and Figures 2017. In: American Cancer Society I, editor. Atlanta2017. p. 1-56.

2. Society AC. Breast Cancer Facts and Figures 2015-2016. Atlanta: American Cancer Society, Inc; 2016.

3. Anderson WF, Rosenberg PS, Prat A, Perou CM, Sherman ME. How many etiological subtypes of breast cancer: two, three, four, or more? Journal of the National Cancer Institute. 2014;106(8):1-11. Epub August 12, 2014.

4. Sledge GW. Anti-vascular endothelial growth factor therapy in breast cancer: game over? J Clin Oncol. 2015;33(2):133-5. Epub 2014/10/27. doi: 10.1200/JCO.2014.58.1298. PubMed PMID: 25349299.

5. Aalders KC, Tryfonidis K, Senkus E, Cardoso F. Anti-angiogenic treatment in breast cancer: Facts, successes, failures and future perspectives. Cancer Treat Rev. 2017;53:98-110. Epub 2017/01/03. doi: 10.1016/j.ctrv.2016.12.009. PubMed PMID: 28088074.

6. Kristensen TB, Knutsson ML, Wehland M, Laursen BE, Grimm D, Warnke E, Magnusson NE. Anti-vascular endothelial growth factor therapy in breast cancer. Int J Mol Sci. 2014;15(12):23024-41. Epub 2014/12/11. doi: 10.3390/ijms 151223024. PubMed PMID: 25514409; PMCID: PMC4284752.

7. Society AC. Breast Cancer Facts and Figures 2015-2016. Atlanta: American Cancer Society, Inc; 2015.

8. Sørlie T, Perou CM, Tibshirani R, Aas T, Geisler S, Johnsen H, Hastie T, Eisen MB, van de Rijn M, Jeffrey SS, Thorsen T, Quist H, Matese JC, Brown PO, Botstein D, Lønning PE, Børresen-Dale AL. Gene expression patterns of breast carcinomas distinguish tumor subclasses with clinical implications. Proc Natl Acad Sci U S A. 2001;98(19):10869-74. doi: 10.1073/pnas.191367098. PubMed PMID: 11553815; PMCID: PMC58566.

9. Fan C, Oh DS, Wessels L, Weigelt B, Nuyten DS, Nobel AB, van't Veer LJ, Perou CM. Concordance among gene-expression-based predictors for breast cancer. N Engl J Med. 2006;355(6):560-9. doi: 10.1056/NEJMoa052933. PubMed PMID: 16899776.

10. Carey LA, Dees EC, Sawyer L, Gatti L, Moore DT, Collichio F, Ollila DW, Sartor CI, Graham ML, Perou CM. The triple negative paradox: primary tumor chemosensitivity of breast cancer subtypes. Clin Cancer Res. 2007;13(8):2329-34. doi: 10.1158/1078-0432.CCR-06-1109. PubMed PMID: 17438091.

11. Takeuchi H, Kawanaka H, Fukuyama S, Kubo N, Hiroshige S, Yano T. Comparison of the prognostic values of preoperative inflammation-based parameters in patients with breast cancer. PLoS One. 2017;12(5):e0177137. Epub 2017/05/10. doi: 10.1371/journal.pone.0177137. PubMed PMID: 28489884.

12. Grivennikov SI, Greten FR, Karin M. Immunity, inflammation, and cancer. Cell. 2010;140(6):883-99. doi: 10.1016/j.cell.2010.01.025. PubMed PMID: 20303878; PMCID: PMC2866629.

13. Walter M, Liang S, Ghosh S, Hornsby PJ, Li R. Interleukin 6 secreted from adipose stromal cells promotes migration and invasion of breast cancer cells. 
Oncogene. 2009;28(30):2745-55. doi: 10.1038/onc.2009.130. PubMed PMID: 19483720; PMCID: 2806057.

14. Bolin C, Tawara K, Sutherland C, Redshaw J, Aranda P, Moselhy J, Anderson R, Jorcyk CL. Oncostatin m promotes mammary tumor metastasis to bone and osteolytic bone degradation. Genes Cancer. 2012;3(2):117-30. doi: 10.1177/1947601912458284. PubMed PMID: 23050044; PMCID: PMC3463924.

15. Jorcyk CL, Holzer RG, Ryan RE. Oncostatin M induces cell detachment and enhances the metastatic capacity of T-47D human breast carcinoma cells. Cytokine. 2006;33(6):323-36. doi: 10.1016/j.cyto.2006.03.004. PubMed PMID: WOS:000238464800004.

16. Luo Q, Wang C, Jin G, Gu D, Wang N, Song J, Jin H, Hu F, Zhang Y, Ge T, Huo X, Chu W, Shu H, Fang J, Yao M, Gu J, Cong W, Qin W. LIFR functions as a metastasis suppressor in hepatocellular carcinoma by negatively regulating phosphoinositide 3-kinase/AKT pathway. Carcinogenesis. 2015;36(10):1201-12. doi: 10.1093/carcin/bgv108. PubMed PMID: 26249360.

17. Humbert L, Ghozlan M, Canaff L, Tian J, Lebrun JJ. The leukemia inhibitory factor (LIF) and p21 mediate the TGF $\beta$ tumor suppressive effects in human cutaneous melanoma. BMC Cancer. 2015;15:200. doi: 10.1186/s12885-015-1177-1. PubMed PMID: 25885043; PMCID: PMC4389797.

18. Chen D, Sun Y, Wei Y, Zhang P, Rezaeian AH, Teruya-Feldstein J, Gupta S, Liang H, Lin HK, Hung MC, Ma L. LIFR is a breast cancer metastasis suppressor upstream of the Hippo-YAP pathway and a prognostic marker. Nat Med. 2012;18(10):1511-7. doi: 10.1038/nm.2940. PubMed PMID: 23001183; PMCID: PMC3684419.

19. García-Tuñón I, Ricote M, Ruiz A, Fraile B, Paniagua R, Royuela M. OSM, LIF, its receptors, and its relationship with the malignance in human breast carcinoma (in situ and in infiltrative). Cancer Invest. 2008;26(3):222-9. doi: 10.1080/07357900701638491. PubMed PMID: 18317962.

20. Yang X, Lin A, Jiang N, Yan H, Ni Z, Qian J, Fang W. Interleukin-6 trans-signalling induces vascular endothelial growth factor synthesis partly via Janus kinases-STAT3 pathway in human mesothelial cells. Nephrology (Carlton). 2017;22(2):150-8. doi: 10.1111/nep.12746. PubMed PMID: 26869278.

21. Vasse M, Pourtau J, Trochon V, Muraine M, Vannier JP, Lu H, Soria J, Soria C. Oncostatin M induces angiogenesis in vitro and in vivo. Arterioscler Thromb Vasc Biol. 1999;19(8):1835-42. PubMed PMID: 10446061.

22. Winder DM, Chattopadhyay A, Muralidhar B, Bauer J, English WR, Zhang X, Karagavriilidou K, Roberts I, Pett MR, Murphy G, Coleman N. Overexpression of the oncostatin $\mathrm{M}$ receptor in cervical squamous cell carcinoma cells is associated with a pro-angiogenic phenotype and increased cell motility and invasiveness. The Journal of pathology. 2011;225(3):448-62. PubMed PMID: 21952923.

23. Liu J, Hadjokas N, Mosley B, Estrov Z, Spence MJ, Vestal RE. Oncostatin $\mathrm{M}$-specific receptor expression and function in regulating cell proliferation of normal and malignant mammary epithelial cells. Cytokine. 1998;10(4):295-302. doi: S10434666(97)90283-0 [pii] 10.1006/cyto.1997.0283. PubMed PMID: 9617575.

24. Murakami M, Hibi M, Nakagawa N, Nakagawa T, Yasukawa K, Yamanishi K, Taga T, Kishimoto T. IL-6-induced homodimerization of gp130 and 
associated activation of a tyrosine kinase. Science. 1993;260(5115):1808-10. PubMed PMID: 8511589.

25. Boulanger MJ, Garcia KC. Shared cytokine signaling receptors: structural insights from the gp130 system. Adv Protein Chem. 2004;68:107-46. doi: 10.1016/S0065-3233(04)68004-1. PubMed PMID: 15500860.

26. Demyanets S, Huber K, Wojta J. Vascular effects of glycoprotein130 ligands--part I: pathophysiological role. Vascul Pharmacol. 2012;56(1-2):34-46. doi: 10.1016/j.vph.2011.12.004. PubMed PMID: 22197898.

27. Hibi M, Nakajima K, Hirano T. IL-6 cytokine family and signal transduction: a model of the cytokine system. J Mol Med (Berl). 1996;74(1):1-12. PubMed PMID: 8834766.

28. Li TM, Wu CM, Huang HC, Chou PC, Fong YC, Tang CH. Interleukin-11 increases cell motility and up-regulates intercellular adhesion molecule-1 expression in human chondrosarcoma cells. J Cell Biochem. 2012;113(11):3353-62. doi: 10.1002/jcb.24211. PubMed PMID: 22644863.

29. Scheller J, Chalaris A, Schmidt-Arras D, Rose-John S. The pro- and antiinflammatory properties of the cytokine interleukin-6. Biochimica et Biophysica Acta (BBA) - Molecular Cell Research. 2011;1813(5):878-88. doi: http://dx.doi.org/10.1016/j.bbamcr.2011.01.034.

30. Queen MM, Ryan RE, Holzer RG, Keller-Peck CR, Jorcyk CL. Breast cancer cells stimulate neutrophils to produce oncostatin M: Potential implications for tumor progression. Cancer Research. 2005;65(19):8896-904. doi: 10.1158/00085472.can-05-1734. PubMed PMID: ISI:000232199400043.

31. Fischer P, Hilfiker-Kleiner D. Survival pathways in hypertrophy and heart failure: the gp130-STAT axis. Basic Res Cardiol. 2007;102(5):393-411. PubMed PMID: 17918316.

32. Heinrich PC, Behrmann I, Haan S, Hermanns HM, Müller-Newen G, Schaper F. Principles of interleukin (IL)-6-type cytokine signalling and its regulation. Biochem J. 2003;374(Pt 1):1-20. doi: 10.1042/BJ20030407. PubMed PMID: 12773095; PMCID: PMC1223585.

33. Pflanz S, Timans JC, Cheung J, Rosales R, Kanzler H, Gilbert J, Hibbert L, Churakova T, Travis M, Vaisberg E, Blumenschein WM, Mattson JD, Wagner JL, To W, Zurawski S, McClanahan TK, Gorman DM, Bazan JF, de Waal Malefyt R, Rennick D, Kastelein RA. IL-27, a heterodimeric cytokine composed of EBI3 and p28 protein, induces proliferation of naive CD4+ T cells. Immunity. 2002;16(6):779-90. PubMed PMID: 12121660.

34. Scheller J, Grötzinger J, Rose-John S. Updating interleukin-6 classic- and trans-signaling. Signal Transduction. 2006;6(4):240-59. doi: 10.1002/sita.200600086.

35. Kareva I, Abou-Slaybi A, Dodd O, Dashevsky O, Klement GL. Normal Wound Healing and Tumor Angiogenesis as a Game of Competitive Inhibition. PLoS One. 2016;11(12):e0166655. Epub 2016/12/09. doi: 10.1371/journal.pone.0166655. PubMed PMID: 27935954; PMCID: PMC5147849.

36. Hanahan D, Folkman J. Patterns and emerging mechanisms of the angiogenic switch during tumorigenesis. Cell. 1996;86(3):353-64. PubMed PMID: 8756718 . 
37. Dvorak HF, Nagy JA, Feng D, Brown LF, Dvorak AM. Vascular permeability factor/vascular endothelial growth factor and the significance of microvascular hyperpermeability in angiogenesis. Curr Top Microbiol Immunol. 1999;237:97-132. PubMed PMID: 9893348.

38. Ferrara N, Adamis AP. Ten years of anti-vascular endothelial growth factor therapy. Nature reviews Drug discovery. 2016;15(6):385-403. doi: 10.1038/nrd.2015.17. PubMed PMID: 26775688.

39. Bry M, Kivela R, Leppanen VM, Alitalo K. Vascular endothelial growth factor-B in physiology and disease. Physiological reviews. 2014;94(3):779-94. doi: 10.1152/physrev.00028.2013. PubMed PMID: 24987005.

40. Lange C, Storkebaum E, de Almodovar CR, Dewerchin M, Carmeliet P. Vascular endothelial growth factor: a neurovascular target in neurological diseases. Nature reviews Neurology. 2016;12(8):439-54. doi: 10.1038/nrneurol.2016.88. PubMed PMID: 27364743.

41. Brat DJ, Kaur B, Van Meir EG. Genetic modulation of hypoxia induced gene expression and angiogenesis: relevance to brain tumors. Front Biosci. 2003;8:d10016. Epub 2003/01/01. PubMed PMID: 12456339.

42. Niu G, Wright KL, Huang M, Song L, Haura E, Turkson J, Zhang S, Wang T, Sinibaldi D, Coppola D, Heller R, Ellis LM, Karras J, Bromberg J, Pardoll D, Jove R, Yu H. Constitutive Stat3 activity up-regulates VEGF expression and tumor angiogenesis. Oncogene. 2002;21(13):2000-8. doi: 10.1038/sj.onc.1205260. PubMed PMID: 11960372.

43. Damert A, Ikeda E, Risau W. Activator-protein-1 binding potentiates the hypoxia-induciblefactor-1-mediated hypoxia-induced transcriptional activation of vascular-endothelial growth factor expression in C6 glioma cells. Biochem J. 1997;327 ( Pt 2):419-23. PubMed PMID: 9359410; PMCID: PMC1218810.

44. Pang L, Zhang Y, Yu Y, Zhang S. Resistin promotes the expression of vascular endothelial growth factor in ovary carcinoma cells. Int J Mol Sci. 2013;14(5):9751-66. doi: 10.3390/ijms14059751. PubMed PMID: 23652833; PMCID: PMC3676810.

45. Sun D, Liu WJ, Guo K, Rusche JJ, Ebbinghaus S, Gokhale V, Hurley LH. The proximal promoter region of the human vascular endothelial growth factor gene has a G-quadruplex structure that can be targeted by G-quadruplex-interactive agents. Mol Cancer Ther. 2008;7(4):880-9. doi: 10.1158/1535-7163.MCT-07-2119. PubMed PMID: 18413801; PMCID: PMC2367258.

46. Akiri G, Nahari D, Finkelstein Y, Le SY, Elroy-Stein O, Levi BZ. Regulation of vascular endothelial growth factor (VEGF) expression is mediated by internal initiation of translation and alternative initiation of transcription. Oncogene. 1998;17(2):227-36. doi: 10.1038/sj.onc.1202019. PubMed PMID: 9674707.

47. Weiss TW, Simak R, Kaun C, Rega G, Pflüger H, Maurer G, Huber K, Wojta J. Oncostatin M and IL-6 induce u-PA and VEGF in prostate cancer cells and correlate in vivo. Anticancer Res. 2011;31(10):3273-8. PubMed PMID: 21965736.

48. Curtis C, Shah SP, Chin SF, Turashvili G, Rueda OM, Dunning MJ, Speed D, Lynch AG, Samarajiwa S, Yuan Y, Gräf S, Ha G, Haffari G, Bashashati A, Russell R, McKinney S, Langerød A, Green A, Provenzano E, Wishart G, Pinder S, Watson P, Markowetz F, Murphy L, Ellis I, Purushotham A, Børresen-Dale AL, Brenton 
JD, Tavaré S, Caldas C, Aparicio S, Group M. The genomic and transcriptomic architecture of 2,000 breast tumours reveals novel subgroups. Nature.

2012;486(7403):346-52. doi: 10.1038/nature10983. PubMed PMID: 22522925; PMCID: PMC3440846.

49. Voduc KD, Cheang MC, Tyldesley S, Gelmon K, Nielsen TO, Kennecke $\mathrm{H}$. Breast cancer subtypes and the risk of local and regional relapse. J Clin Oncol. 2010;28(10):1684-91. doi: 10.1200/JCO.2009.24.9284. PubMed PMID: 20194857.

50. Lin J, Goldstein L, Nesbit A, Chen MY. Influence of Hormone Receptor Status on Spinal Metastatic Lesions in Patients with Breast Cancer. World Neurosurg. 2015. doi: 10.1016/j.wneu.2015.07.068. PubMed PMID: 26260940.

51. Knight WA, Osborne CK, Yochmowitz MG, McGuire WL. Steroid hormone receptors in the management of human breast cancer. Ann Clin Res. 1980;12(5):202-7. PubMed PMID: 7015982.

52. Badache A, Hynes NE. Interleukin 6 inhibits proliferation and, in cooperation with an epidermal growth factor receptor autocrine loop, increases migration of T47D breast cancer cells. Cancer Res. 2001;61(1):383-91. PubMed PMID: 11196191.

53. Korzus E, Nagase H, Rydell R, Travis J. The mitogen-activated protein kinase and JAK-STAT signaling pathways are required for an oncostatin M-responsive element-mediated activation of matrix metalloproteinase 1 gene expression. J Biol Chem. 1997;272(2):1188-96. PubMed PMID: 8995420.

54. Hurst SM, McLoughlin RM, Monslow J, Owens S, Morgan L, Fuller GM, Topley N, Jones SA. Secretion of oncostatin M by infiltrating neutrophils: regulation of IL-6 and chemokine expression in human mesothelial cells. J Immunol. 2002;169(9):5244-51. PubMed PMID: 12391243.

55. Song Z, Lin Y, Ye X, Feng C, Lu Y, Yang G, Dong C. Expression of IL$1 \alpha$ and IL-6 is Associated with Progression and Prognosis of Human Cervical Cancer. Med Sci Monit. 2016;22:4475-81. PubMed PMID: 27866212; PMCID: PMC5120643.

56. Lin S, Gan Z, Han K, Yao Y, Min D. Interleukin-6 as a prognostic marker for breast cancer: a meta-analysis. Tumori. 2015;101(5):535-41. doi: 10.5301/tj.5000357. PubMed PMID: 26045123.

57. Chang Q, Bournazou E, Sansone P, Berishaj M, Gao SP, Daly L, Wels J, Theilen T, Granitto S, Zhang X, Cotari J, Alpaugh ML, de Stanchina E, Manova K, Li M, Bonafe M, Ceccarelli C, Taffurelli M, Santini D, Altan-Bonnet G, Kaplan R, Norton L, Nishimoto N, Huszar D, Lyden D, Bromberg J. The IL-6/JAK/Stat3 feed-forward loop drives tumorigenesis and metastasis. Neoplasia. 2013;15(7):848-62. PubMed PMID: 23814496 ; PMCID: PMC3689247.

58. Bockhorn J, Dalton R, Nwachukwu C, Huang S, Prat A, Yee K, Chang YF, Huo D, Wen Y, Swanson KE, Qiu T, Lu J, Park SY, Dolan ME, Perou CM, Olopade OI, Clarke MF, Greene GL, Liu H. MicroRNA-30c inhibits human breast tumour chemotherapy resistance by regulating TWF1 and IL-11. Nat Commun. 2013;4:1393. doi: 10.1038/ncomms2393. PubMed PMID: 23340433; PMCID: PMC3723106.

59. Lewis VO, Ozawa MG, Deavers MT, Wang G, Shintani T, Arap W, Pasqualini R. The interleukin-11 receptor alpha as a candidate ligand-directed target in osteosarcoma: consistent data from cell lines, orthotopic models, and human tumor samples. Cancer Res. 2009;69(5):1995-9. doi: 10.1158/0008-5472.CAN-08-4845. PubMed PMID: 19244100. 
60. Ryan RE, Martin B, Mellor L, Jacob RB, Tawara K, McDougal OM, Oxford JT, Jorcyk CL. Oncostatin M binds to extracellular matrix in a bioactive conformation: implications for inflammation and metastasis. Cytokine. 2015;72(1):71-85. doi: 10.1016/j.cyto.2014.11.007. PubMed PMID: 25622278; PMCID: PMC4328881.

61. Sa-Nguanraksa D, Chuangsuwanich T, Pongpruttipan T, O-Charoenrat P. High vascular endothelial growth factor gene expression predicts poor outcome in patients with non-luminal A breast cancer. Mol Clin Oncol. 2015;3(5):1103-8. doi: 10.3892/mco.2015.574. PubMed PMID: 26623059; PMCID: PMC4534845.

62. Linardou H, Kalogeras KT, Kronenwett R, Alexopoulou Z, Wirtz RM, Zagouri F, Scopa CD, Gogas H, Petraki K, Christodoulou C, Pavlakis K, Koutras AK, Samantas E, Patsea H, Pectasides D, Bafaloukos D, Fountzilas G. Prognostic Significance of VEGFC and VEGFR1 mRNA Expression According to HER2 Status in Breast Cancer: A Study of Primary Tumors from Patients with High-risk Early Breast Cancer Participating in a Randomized Hellenic Cooperative Oncology Group Trial. Anticancer Res. 2015;35(7):4023-36. PubMed PMID: 26124351.

63. Linardou H, Kalogeras KT, Kronenwett R, Kouvatseas G, Wirtz RM, Zagouri F, Gogas H, Christodoulou C, Koutras AK, Samantas E, Pectasides D, Bafaloukos D, Fountzilas G. The prognostic and predictive value of mRNA expression of vascular endothelial growth factor family members in breast cancer: a study in primary tumors of high-risk early breast cancer patients participating in a randomized Hellenic Cooperative Oncology Group trial. Breast Cancer Res. 2012;14(6):R145. doi: 10.1186/bcr3354. PubMed PMID: 23146280; PMCID: PMC4053134.

64. Fountzilas G, Dafni U, Papadimitriou C, Timotheadou E, Gogas H, Eleftheraki AG, Xanthakis I, Christodoulou C, Koutras A, Papandreou CN, Papakostas P, Miliaras S, Markopoulos C, Dimitrakakis C, Korantzopoulos P, Karanikiotis C, Bafaloukos D, Kosmidis P, Samantas E, Varthalitis I, Pavlidis N, Pectasides D, Dimopoulos MA. Dose-dense sequential adjuvant chemotherapy followed, as indicated, by trastuzumab for one year in patients with early breast cancer: first report at 5-year median follow-up of a Hellenic Cooperative Oncology Group randomized phase III trial. BMC Cancer. 2014;14:515. doi: 10.1186/1471-2407-14-515. PubMed PMID: 25026897; PMCID: PMC4223601.

65. Ellis LM, Hicklin DJ. VEGF-targeted therapy: mechanisms of anti-tumour activity. Nature reviews Cancer. 2008;8(8):579-91. doi: 10.1038/nrc2403. PubMed PMID: 18596824.

66. West NR, Murphy LC, Watson PH. Oncostatin M suppresses oestrogen receptor- $\alpha$ expression and is associated with poor outcome in human breast cancer. Endocr Relat Cancer. 2012;19(2):181-95. doi: 10.1530/ERC-11-0326. PubMed PMID: 22267707.

67. Pawlus MR, Wang L, Hu CJ. STAT3 and HIF1 $\alpha$ cooperatively activate HIF1 target genes in MDA-MB-231 and RCC4 cells. Oncogene. 2014;33(13):1670-9. doi: 10.1038/onc.2013.115. PubMed PMID: 23604114; PMCID: PMC3868635.

68. Asgeirsson KS, Olafsdóttir K, Jónasson JG, Ogmundsdóttir HM. The effects of IL-6 on cell adhesion and e-cadherin expression in breast cancer. Cytokine. 1998;10(9):720-8. doi: 10.1006/cyto.1998.0349. PubMed PMID: 9770334.

69. Hao Y, Kuang Z, Jing J, Miao J, Mei LY, Lee RJ, Kim S, Choe S, Krause DC, Lau GW. Mycoplasma pneumoniae modulates STAT3-STAT6/EGFR-FOXA2 
signaling to induce overexpression of airway mucins. Infect Immun. 2014;82(12):524655. doi: 10.1128/IAI.01989-14. PubMed PMID: 25287927; PMCID: PMC4249270.

70. Lamoreaux WJ, Fitzgerald ME, Reiner A, Hasty KA, Charles ST.

Vascular endothelial growth factor increases release of gelatinase A and decreases release of tissue inhibitor of metalloproteinases by microvascular endothelial cells in vitro. Microvasc Res. 1998;55(1):29-42. doi: 10.1006/mvre.1997.2056. PubMed PMID: 9473407. 
CHAPTER THREE: ONCOSTATIN M PROMOTES BREAST CANCER METASTASIS TO LUNG AND INCREASES CIRCULATING TUMOR CELL NUMBER

\author{
Authors \\ Ken Tawara*1 ${ }^{1}$, Celeste Bolin*1 ${ }^{1}$, Jordan Koncinsky*1, Sujatha Kadaba ${ }^{1}$, Hunter \\ Covert $^{1}$, Caleb Sutherland ${ }^{1}$, Laura Bond ${ }^{1}$, Joseph Kronz ${ }^{2}$, Joel Garbow ${ }^{3}$, and Cheryl L. \\ Jorcyk $^{1+}$ \\ ${ }^{1}$ Boise State University, Boise, Idaho 83725; ${ }^{2}$ Medical Center, Nampa, ID, \\ ${ }^{3}$ Washington University School of Medicine, St. Louis, MO
}

\begin{abstract}
Systemic and chronic inflammatory conditions reduce patient survival and have been associated with increased breast cancer aggressiveness. This paper characterizes the role of an inflammatory cytokine oncostatin $\mathrm{M}(\mathrm{OSM})$ and its role in the preintravasation aspects of breast cancer metastasis. First, we established the expression pattern of OSM in human breast cancer tissue using tissue microarray analysis and found that OSM was expressed at highest levels in ductal carcinoma in situ (DCIS) when
\end{abstract}


compared to invasive ductal carcinoma (IDC), lymph node metastasis, or adjacent normal tissue. This finding suggests that OSM may be operant during earlier steps of breast cancer metastasis. To investigate this further, both xenograft and syngeneic orthotopic mouse models of breast cancer were utilized. In mice bearing MDA-MB-231-Luc2 tumors, injection of rhOSM not only increased metastases to the lung and decreased survival, but also increased circulating tumor cell (CTC) numbers. This is the first time that a gp130 family inflammatory cytokine has been shown to directly affect the amount of tumor cells found in circulation. These findings are corroborated using a 4T1.2 syngeneic mouse model of breast cancer. Mice bearing 4T1.2-shOSM tumors with KD tumor expression of OSM had reduced CTCs, lowered lung metastatic burden, and increased survival compared to mice bearing control tumors. CTC numbers were further reduced in OSM knockout mice bearing the same tumors, demonstrating the importance of both paracrine- and autocrine-produced OSM in this process. On the other hand, after bypassing initial steps of metastases by injecting the $4 \mathrm{~T} 1.2$ cancer cells directly into the circulatory system, OSM did not affect survival. During in vitro studies, OSM induced both 4T1.2 tumor cell detachment and migration. Collectively, we suggest that OSM affects the early steps of metastatic breast cancer progression and that suppression of OSM may help prevent breast cancer metastasis.

\section{Introduction}

The inflammatory gp130 family of cytokines has been shown to modulate immune function (1) with important implications in tumor immunology (2). Inflammation and inflammatory cytokines have been associated with increased breast cancer metastasis and poor survival rates (3-6). Interleukin-6 (IL-6), as a well-known 
inflammatory cytokine in the gp130 family, promotes breast cancer metastasis but therapies targeted against it have failed in clinical trials $(7,8)$. Other cytokines in the gp130 family such as oncostatin M (OSM) also modulate inflammation, and OSM has been associated with a wide variety of disease states such as in inflammatory bowel disease, arthritis, gingivitis, and lupus nephritis (9-12). In the context of cancer, OSM has been shown to induce in vitro metrics associated with cancer invasiveness and promote breast cancer metastasis to bone (13-17). In the breast tumor microenvironment (TME), OSM is produced by breast tumor cells (13), as well as stromal cells such as tumorassociated macrophages and neutrophils and adipose tissues (18-20). After OSM is secreted, it binds to and accumulates in the extracellular matrix (ECM) in an active form, which may lead to chronic local inflammation and increased tumor metastasis (21). Specifically, it has been shown that human breast tumor cells signal neutrophils to secrete OSM, which subsequently induces tumor cell vascular endothelial growth factor (VEGF) production, cell detachment, and invasive capacity (18). Collectively, these studies suggest that OSM functions in breast cancer progression both in an autocrine and paracrine fashion.

OSM signaling utilizes two receptors. OSM binds with high affinity to the OSM receptor (OSMR) (gp130 subunit + OSMR $\beta$ ) and with lower affinity to the leukemia inhibitory factor receptor (LIFR) (gp130 + LIFR $\beta)(22)$. While OSM binding to the OSMR has been shown to promote cancer cell malignancy and reduce long-term survival in breast cancer patients (23), activation of the LIFR appears to suppress tumor growth and metastasis (24). OSMR signaling initiates the JAK/STAT, MAPK, and PI3K/AKT 
pathways (25-27), as well as the stress-activated mitogen-activated protein kinases p38 and JNK pathways (28).

As a pleiotropic cytokine, OSM seems to play an important role in promoting breast cancer metastatic potential in vitro while inhibiting breast tumor cell proliferation $(10,29,30)$. OSM has been shown to function on breast and various other cancer cells in culture to: i) promote an epithelial-mesenchymal transition (EMT) and a stem cell-like phenotype $(27,31,32)$, ii) upregulate expression of proteases such as matrix metalloproteinases (MMPs) $(10,15)$, iii) promote tumor cell detachment and subsequent invasion $(15,16,33)$, iv) induce the expression of VEGF, hypoxia inducible factor 1 alpha (HIF $1 \alpha$ ), and other proangiogenic factors (33-36), and v) suppress estrogen receptor alpha (ER $\alpha$ ) expression, (23). Despite increasing evidence in vitro, limited studies have addressed the role of OSM in breast cancer metastasis in vivo.

Our lab's previous studies were the first to show the importance of OSM in breast cancer metastasis to bone. Specifically, reduced tumor cell-produced OSM expression led to a decrease in osteolytic bone metastasis in an orthotopic 4T1.2 mouse model (17). Along with earlier studies demonstrating OSM's function in normal bone homeostasis $(37,38)$, this work suggests an important role for OSM during post-intravasation breast cancer metastasis to bone and subsequent bone destruction. While in vitro studies suggest that OSM promotes the early steps of the metastatic cascade, no extant work differentiates between OSM's impact on pre- versus post-intravasation aspects of the breast cancer metastatic cascade.

This paper demonstrates that OSM initiates pre-intravasation steps of the metastasis cascade, increasing CTC numbers and lung metastases and decreasing 
survival. Conversely, we also show that OSM had no effect on mouse survival in the post-intravasation model that bypassed the early steps of metastasis by injecting tumor cells directly into circulation. Therefore, our work suggests that therapeutic suppression of OSM in the tumor microenvironment might not only be an effective treatment strategy for bone metastasis; it could also be used as a preventative therapeutic to mitigate overall breast cancer metastasis.

\section{Materials and Methods}

\section{$\underline{\text { Tissue microarrays }}$}

Breast tissue from 72 patients was obtained from paraffin block archives at the Department of Pathology, Mercy Medical Center, Nampa Idaho. Three tissue microarrays (TMA) of $1 \mathrm{~mm}$ thickness were assessed. The TMAs were stained for OSM using the Histostain Kit (Cat \#95-9843; Invitrogen, Carlsbad, CA) per manufacturer's instructions. The TMAs were deparaffinized and stained overnight with 1:400 dilution of rabbit antihuman OSM primary antibody (Cat \#sc-129; Santa Cruz Biotechnology, Santa Cruz, CA) and 1 hour with 1:1000 goat-anti rabbit IgG-AP $2^{\circ}$ antibody. TMAs stained with secondary antibody alone served as the negative control, and spleen and salivary gland served as positive controls for OSM staining.

The TMAs were analyzed in multiple sets of random orders for OSM expression intensity by a pathologist graded as follows: $0=$ No staining; $1=$ Light staining; $2=$ Medium staining; 3=Dark staining. Gradings for each patient were averaged for each cell tissue type (ductal epithelial, vessel, stroma). Additional methods are detailed in the Supplemental Materials and Methods. 


\section{$\underline{\text { Cell lines and culture conditions }}$}

MDA-MB-231 D3H2LN luc2 cells (Caliper Life Sciences); and MDA-MB-231 (ATCC) human breast cancer cells were cultured in RMPI media supplemented with $10 \%$ FBS and 100 units $/ \mathrm{mL}$ of penicillin and streptomycin. Cells were maintained at $37^{\circ} \mathrm{C}$, $5 \%$ carbon dioxide, and $95 \%$ humidity. $4 \mathrm{~T} 1.2$ mouse mammary cancer cells were cultured in alpha-MEM media supplemented with 10\% fetal bovine serum (FBS), $1 \mathrm{mM}$ sodium pyruvate, and 100 units $/ \mathrm{mL}$ each of penicillin and streptomycin, and passaged for no more than 6 months. All media and supplements were obtained from Hyclone (Logan, UT).

\section{$\underline{\text { Plasmid construct design and cell transfections }}$}

To transduce MDA-MB-231-Luc2 D3H2LN cells with a tetracycline (TET)inducible vector, the full-length OSM cDNA was cloned into the pLenti 6.3/TO/V5DEST vector. Lentiviral transduction of the pLenti 6.3/TO/V5-DEST+hOSM vector and pLenti3.3/TR vector was performed using the ViraPower ${ }^{\mathrm{TM}}$ II Lentiviral Gateway ${ }^{\circledR}$ Expression System (Life technologies, K367-20) in accordance with manufacturer's instructions. Stably transduced cell lines were tested for TET induction of hOSM expression by ELISA and Western Blot.

To create OSM knockdown 4T1.2 cells, OSM shRNA and a LacZ shRNA sequences were cloned into the pSilencer 4.1 plasmid and stably transfected into 4T1.2 cells as previously described (17).

\section{$\underline{\text { ELISA }}$}

OSM produced by tetracycline (TET)-inducible MDA-MB-231 (MDA ${ }^{\mathrm{TO} / \mathrm{OSM}}$ ) cells were tested for in vitro activity. $\mathrm{MDA}^{\mathrm{TO} / \mathrm{OSM}}$ cells were treated with $0.1 \mathrm{mg} / \mathrm{mL}$ 
tetracycline for 48 hours to generate conditioned media (CM) containing OSM. The CM was then applied to parental MDA-MB-231, MDA-MB-231-Luc2, or T47D cells for 30 minutes. Respective cell lysates were then collected from treated cells using the PathScan® Sandwich ELISA Lysis Buffer (Cell Signaling, Cat\# 7018). The lysates were then run on a PathScan ${ }^{\circledR}$ Phospho-Stat3 (Tyr705) Sandwich ELISA in accordance with the manufacturer's instructions (Cell Signaling, Cat\# 7146).

To assess OSM concentration in animal serum, whole blood was collected from $\left(\mathrm{MDA}^{\mathrm{TO} / \mathrm{OSM}}\right)$ tumor xenograft animals at the experimental endpoint and allowed to coagulate for 30 minutes. The coagulated blood was centrifuged at 2,500 RPM for 10 minutes and the upper layer collected as serum. The serum was then diluted 1:3 in PBS and used in the hOSM ELISA (R\&D systems, DY295), which was performed in accordance with manufacturer's instructions.

$\underline{\text { Western blot analysis }}$

OSM was induced in $\mathrm{MDA}^{\mathrm{TO} / O S M}$ cells for 48 hours with $0.1 \mathrm{mg} / \mathrm{mLtetracycline}$ in 10\% FBS RPMI 1640 media. The CM was collected, run on a gel, and blotted onto $0.22 \mu \mathrm{M}$ PVDF membranes (Millipore). Membranes were blocked using 5\% nonfat dry milk (NFDM) diluted in PBS at pH 7.4 with $0.05 \%$ Tween-20. Anti-human OSM antibody (Santa Cruz Biotechnology, Cat\# sc-129) was used at 1:1000 dilution in 5\% NFDM-PBS-T, and a secondary anti-rabbit-HRP (Jackson Immunologicals, Cat\# 711035-152) was used at 1:5000 dilution in 5\% NFDM- PBS-T.

\section{$\underline{\text { Animals and tumor cell injections }}$}

Six-week-old female athymic nude mice were used for the xenograft experiments, and six-week-old female Balb/c mice were used for the syngeneic studies. All mice were 
obtained from the National Cancer Institute's Animal Production Facility (Frederick, MD). OSM knockout Balb/c mice were backcrossed from OSM knockout C57BL/6 mice that were a kind gift from Dr. Peter Donovan, indirectly, through Dr. James Ihle (St. Jude's Children's Hospital, Memphis, TN). Animals were backcrossed for at least 10 generations and genotyping was done at each generation to ensure the presence of the knockout allele. Non-surgical orthotopic injections were performed as described previously with $2.0 \times 10^{6}$ cells diluted in $50 \mu \mathrm{L}$ of PBS containing $10 \%$ medium for the xenograft model, and $1 \times 10^{5}$ cells for the syngeneic models. (17). For all animals, starting at 2 weeks post-injection, tumor length and width were measured by mechanical calipers 3 times a week and tumor volume was extrapolated using the following equation (tumor volume $=($ length $\mathrm{x}$ width`2) $/ 2)$. "Survival endpoint" was defined by the IACUC as tumor size greater than $20 \mathrm{~mm}$ in diameter, $10 \%$ or more weight loss, and/or appearance of cachexia. At the experimental endpoint animals were sacrificed, their organs harvested and examined for any abnormalities. Further analysis specific to each model is described below.

For peri-tumoral OSM injections, either $50 \mu \mathrm{L}$ PBS or $1 \mu \mathrm{g}$ recombinant fulllength human OSM (Peprotech) diluted in $50 \mu \mathrm{L}$ PBS was injected into the area surrounding the tumor three times per week until the end point of the experiment. When the tumors became palpable, mice were randomized into groups and began receiving pertumoral injections.

For the TET-OSM-inducible MDA-MB-231 (MDA $\left.{ }^{\mathrm{TO} / \mathrm{OSM}}\right)$ experiments, the OSM-induced group was given $2 \%$ sucrose water containing $0.1 \mathrm{mg} / \mathrm{mL}$ tetracycline, while the control mice were given just $2 \%$ sucrose water until the endpoint of the 
experiment. To assess blood platelet numbers, blood was collected at endpoint into EDTA-coated blood sample tubes (Cat\# BD Biosciences, Franklin Lakes, NJ) and complete blood count (CBC) analysis was performed by WestVet Veterinary Clinic (5024 W Chinden Blvd, Garden City, ID).

$\underline{\text { In vivo bioluminescence imaging and tumor progression }}$

Bioluminescence imaging (BLI) of live animals was initiated at 13 days after cell injection and performed weekly. Three to five mice were imaged at one time. Ex vivo organs were also imaged using BLI. Both procedures follow our previously described protocols (39).

Detection of circulating tumor cells (Alu qPCR)

The detection of human circulating tumor cells in mouse blood was performed as described previously (40). A human DNA standard curve was prepared by adding a specified number of human MDA-MB-231 cells into mouse blood, and then the DNA was isolated for use in the qPCR reactions. Genomic DNA was isolated from $100 \mathrm{uL}$ of whole blood collected from mice at the end of the experiment. DNA was isolated using the DNeasy Blood \& Tissue kit (Qiagen Cat\# 69581) using the manufacturer's standard instructions. DNA concentrations were normalized between each sample and $4.5 \mathrm{ng}$ of DNA was added to each $25 \mu \mathrm{L}$ qPCR reaction. The qPCR reaction mixture was obtained from the SYBR GoTaq qPCR Master Mix (Promega, Cat\# TM318, Madison WI), and reaction mixtures were prepared in accordance with manufacturer recommendations. To each reaction, $0.125 \mu \mathrm{L}$ of $100 \mu \mathrm{M}$ human Alu and GAPDH primers were added. The primer sequences used for Alu were (s:CACCTGTAATCCCAGCACTTT a:CCCAGGCTRGGAGTCGCAGT), and for GAPDH the sequence used was (s: 
ATGACATCAAGAAGGTGGTG; a: CATACCAGGAAATGAGCTTG). Reaction conditions were: $50{ }^{\circ} \mathrm{C}$ for 2 minutes, $95{ }^{\circ} \mathrm{C}$ for 3 minutes and 40 cycles of: $\left(95{ }^{\circ} \mathrm{C} 00: 15\right.$, $60{ }^{\circ} \mathrm{C} \mathrm{00:30,72}{ }^{\circ} \mathrm{C} \mathrm{00:30)} \mathrm{and} \mathrm{fluorescence} \mathrm{measurements} \mathrm{were} \mathrm{taken} \mathrm{during} \mathrm{the}$ annealing temperature stage $\left(60^{\circ} \mathrm{C}\right) . \mathrm{C}_{\mathrm{t}}$ values were determined and the final results were normalized to GAPDH signal levels.

\section{Quantitative PCR (qPCR)}

For quantitative analysis of lung metastases, lungs dissected from mice bearing mammary tumors were snap-frozen in liquid nitrogen and pulverized into a fine powder. DNA was extracted using an STE buffer (100 mM NaCl, 10 mM Tris-HCl pH 8.0, $1 \mathrm{mM}$ EDTA) containing $20 \mu \mathrm{g} / \mathrm{mL}$ of proteinase $\mathrm{K}$ and purified by two phenol/chloroform (1:1 v/v) extractions followed by ethanol precipitation. The ratio of cancer cells to normal cells was quantified by measuring the neomycin resistance gene $\left(\right.$ neo $\left.^{r}\right)$ DNA levels versus the vimentin DNA loading control, as described previously (41). Taqman PCR was performed on an Applied Biosystems 7500 real-time thermocycler. Probe and primer sequences are listed in Supplemental Table 1. The cycling conditions were run as follows: $50{ }^{\circ} \mathrm{C}$ for 5 minutes, $95^{\circ} \mathrm{C}$ for 2 minutes, then 40 cycles of $95^{\circ} \mathrm{C}$ for 1 minutes and $60{ }^{\circ} \mathrm{C}$ for 45 seconds. Fluorescence was measured every cycle after the annealing step and threshold cycle number $\left(\mathrm{C}_{\mathrm{t}}\right)$ values were calculated. The data was analyzed using the comparative $\mathrm{DC}_{\mathrm{t}}$ method (42).

\section{In-vivo magnetic resonance imaging}

Respiratory-gated, spin-echo magnetic resonance (MR) images of mice were collected in an Oxford Instruments (Oxford, UK) 4.7 tesla, 40-cm bore magnet. The magnet was equipped with Agilent/Magnex (Yarnton, UK) actively shielded, (21-cm 
inner diameter, $\sim 30 \mathrm{G} / \mathrm{cm}, \sim 200 \mathrm{~ms}$ rise time) gradient coils and International Electric Company (Helsinki, Finland) gradient power amplifiers and interfaced with an Agilent/ Varian NMR Systems (Santa Clara, CA) DirectDrive ${ }^{\mathrm{TM}}$ console. Data was collected using a Stark Contrast (Erlangen, Germany) $2.5 \mathrm{~cm}$ birdcage RF coil. Prior to the imaging experiments, mice were anesthetized with isoflurane and were maintained on isoflurane $/ \mathrm{O}_{2}(1-1.5 \% \mathrm{v} / \mathrm{v})$ throughout data collection. Animal core body temperature was maintained at $37 \pm 1^{\circ} \mathrm{C}$ by circulation of warm air through the bore of the magnet. During the imaging experiments, the respiration rates for all mice were regular and $\sim 2 \mathrm{sec}^{-1}$. Synchronization of MR data collection with animal respiration was achieved with a home-built respiratory-gating unit (43) and all images were collected during postexpiratory periods. Imaging parameters are $\mathrm{TR}=3 \mathrm{~s}, \mathrm{TE}=20 \mathrm{~ms}, \mathrm{FOV}=2.5 \mathrm{~cm}^{2}$, Data matrix $=128 \times 128 ;$ slice thickness $=0.5 \mathrm{~mm}$; number of averages $(\mathrm{NEX})=4$. Lung tumors were manually segmented with ImageJ (rsbweb.nih.gov/ij), and the number and volume of all metastatic tumors were measured and recorded, on an animal-by-animal basis, as described previously (43).

Detection of circulating tumor cells (clonogenic assay)

Colony forming assays were performed to detect circulating tumor cells in mouse blood. At the end point of the animal experiment, whole blood was collected via intracardiac puncture into EDTA-coated tubes. Red blood cells (RBCs) were lysed with RBC lysis solution (155 mM NH $4 \mathrm{Cl}, 10 \mathrm{mM} \mathrm{KHCO}_{3}, 0.1 \mathrm{mM}$ EDTA diluted in $\left.\mathrm{ddH}_{2} \mathrm{O}\right)$ for 4 minutes. The remaining cell mixture, containing white blood cells and circulating tumor cells, was spun down and washed $2 \mathrm{X}$ with PBS. The cell pellet was then resuspended in MEM-alpha with $10 \% \mathrm{FBS}$, and plated and incubated at $37{ }^{\circ} \mathrm{C}$ for $7-10$ 
days until colonies formed. The colonies were then fixed with $10 \%$ formalin in PBS for 15 minutes, stained with coomassie blue and counted.

Epithelial-mesenchymal transition assay

4T1.2 mouse mammary cancer cells were plated on a 6-well plate to a confluence of $30 \%$ in alpha-MEM with $10 \%$ FBS and $1 \%$ penicillin - streptomycin. Following a period of 24 hours to allow for cells to adhere, $25 \mathrm{ng} / \mathrm{mL}$ recombinant mouse OSM (rmOSM) was added to appropriate wells. Photomicrographs were taken at a power of 100x at times of 24 hours and 48 hours to observe phenotypic EMT changes over the 2day period.

\section{Cell migration assay}

4T1.2 cells were plated on 6-well plates to a confluency of $80 \%$ in alpha-MEM with $10 \%$ FBS. After the cells attached overnight, a straight scratch on the cell monolayer was made with a sterile $1000 \mu \mathrm{L}$ polypropylene pipette tip, and loose cells and debris were washed away with three sterile PBS washes. The cells were then treated with or without $25 \mathrm{ng} / \mathrm{mL}$ rmOSM, and imaged every day for 3 days on the same part of the scratch using negative phase contrast microscopy. The images were then imported into Image $(\mathrm{NIH})$, and raw unmigrated area was measured by calculating the number of pixels in the area with no migration. Relative migration intensity was calculated using this formula: Migration intensity $=($ Day 0 unmigrated area / Day n unmigrated area $)-1$

\section{Cell detachment assay}

4T1.2 mouse mammary cancer cells were plated on 24-well tissue culture dishes to a confluency of $80 \%$ in alpha-MEM with $10 \%$ FBS. The cells were allowed to attach overnight, and rmOSM (25 ng/mL, R\&D systems) suspended in alpha-MEM, 10\% FBS 
media was added to the cells. For up to 8 days, cells that were detached were collected and counted by hemocytometer and viable cells were detected by lack of trypan blue (CAT\# SV30084.01, Hyclone) staining.

$\underline{\text { Statistical analysis }}$

Statistical comparisons between multiple groups were assessed by one or two-way ANOVA using Tukey's post-test analysis. Comparisons between two groups were analyzed by student's t-test (two-tailed, unpaired). The statistical analyses were performed by using Prism GraphPad 5.0b software (GraphPad Software Inc., San Diego, CA). Survival data was analyzed using the Log-rank (Mantel-Cox) test. Asterisks denote $* \mathrm{p}<0.05, * * \mathrm{p}<0.01$, or $* * * \mathrm{p}<0.001$

\section{Results}

High OSM expression in breast ductal carcinoma in situ (DCIS) and IDC suggests autocrine signaling

To assess breast epithelial cell expression and location of OSM in human breast tumors, tissue microarrays (TMAs) containing samples from 72 patients were analyzed by immunohistochemistry. Interestingly, staining intensity was highest in the DCIS group compared to normal tissue, while $2^{\circ}$ antibody alone showed little staining (Fig 1A). The mean staining intensity for normal adjacent tissue (1.33) was significantly lower than that of DCIS (2.00) and IDC (1.66) tissues, while metastatic tissue (1.24) was statistically similar to normal tissue (Fig. 1B and Table 1). These results suggest that OSM protein levels are higher in the earlier stages of breast cancer and that tumor cell-produced OSM may be important in autocrine signaling for the promotion of tumor progression. 
$\underline{\text { OSM generated from TET-inducible MDA-MB-231 }\left(\mathrm{MDA}^{\mathrm{TO} / \mathrm{OSM}}\right) \text { cells increases }}$ $\underline{\text { metastases to the lung and decreases survival }}$

In order assess the effect of cancer cell-produced OSM in a tumor microenvironment, we developed a stably-transduced triple negative breast cancer (TNBC) MDA-MB-231-Luc2 D3H2LN cell line that secretes OSM in response to tetracycline treatment (+TET; $\left.\mathrm{MDA}^{\mathrm{TO} / \mathrm{OSM}}\right)$. To compare the OSM produced by the $\mathrm{MDA}^{\mathrm{TO} / O S M}$ cells to recombinant human OSM (hOSM), conditioned media (CM) from MDA $^{\text {TO/OSM }}$ cells treated with TET $(0.1 \mu \mathrm{g} / \mathrm{mL})$ was collected. MDA-MB-231, MDAMB-231-Luc, and T47D breast cancer cells were treated with either CM from $\mathrm{MDA}^{\mathrm{TO} / O S \mathrm{M}}$ cells or with rhOSM $(25 \mathrm{ng} / \mathrm{mL})$ for 30 minutes. OSM signaling was assessed by measuring STAT3 activation using a pSTAT3 ELISA. For each cell line investigated, there were no significant difference in the level of pSTAT3 induced by OSM produced from TET-induced MDA ${ }^{\text {TO/OSM }}$ cells compared to rhOSM (Fig 2A). Furthermore, the CM from MDA ${ }^{\text {TO/OSM }}$ cells treated with TET was assessed on a hOSM immunoblot and an expected size band (26 kDa) was detected (Fig, 2A, inset).

To assess the activity of $\mathrm{MDA}^{\mathrm{TO} / \mathrm{OSM}}$ cells in vivo, $1 \times 10^{6}$ cells were injected into the $4^{\text {th }}$ mammary fat pad of female athymic nude mice. The mice were given drinking water with tetracycline (+TET) or without tetracycline (-TET) $(0.1 \mathrm{mg} / \mathrm{mL}$ in $2 \%$ sucrose water) to induce OSM expression in the cancer cells. At the experimental endpoint, their serum was then separated from whole blood, and serum OSM levels were assessed by ELISA. Tumor bearing mice +TET had a 67-fold higher level of OSM levels present in their serum than -TET mice (Fig 2B, left). Each animal's physical condition was assessed, and animals with $\mathrm{MDA}^{\mathrm{TO} / \mathrm{OSM}}$ tumors + TET had significantly increased blood 
platelet counts (Fig. 2B, center). TET-treated mice also experienced a significant decrease in body weight compared -TET mice (Fig. 2B, right; sFig. 1A), displaying a prominent spinal column and reduced apparent body fat indicative of cachexia (sFig. 1B). In the literature, it has been reported that cachexia, elevated inflammatory factors, and kidney disease may be correlated with each other $(44,45)$, and in this study, the cachexic animals had kidney abnormalities with hypoperfusion and damage to the gross morphological kidney structures (sFig. 1C). This correlated with +TET treatment and high levels of serum OSM in the animals (sFig.1D and E), which suggest that high OSM levels may contribute to the development of cachexia and kidney organ dysfunction. Additionally, in a separate experiment, animals were given TET drinking water for only 1 week to assess early effects of OSM on metastasis. Animals on 1 week of +TET had higher levels of metastases to the lung as assessed by ex vivo imaging compared to-TET mice (Fig. 2C). This result suggests that even short-term elevation in the level of OSM can promote the development of metastases. To measure animal survival, mice with $\mathrm{MDA}^{\mathrm{TO} / \mathrm{OSM}}$ tumors were treated with or without TET and allowed to progress to end point. +TET mice had a mean decreased survival of 11 days compared to -TET mice (Fig. 2D). Collectively, these results demonstrated that elevated levels of tumor cell-produced OSM lead to increased lung metastases, decreased survival, and deterioration in body condition indicative of cachexia.

$\underline{\text { OSM increases metastasis to lung and circulating tumor cell numbers in an orthotopic }}$ $\underline{\text { MDA-MB-231 model of breast cancer }}$

To assess the paracrine effects of OSM, exogenous OSM was injected peritumorally in an orthotopic MDA-MB-231 xenograft model. In this model, 2 x $10^{6}$ MDA- 
MB-231-Luc2 D3H2LN cells were injected into the fourth mammary fat pads of female nude mice. After the tumors were palpable $(\sim 3 \mathrm{~mm})$, OSM $(1 \mu \mathrm{g}$ in $50 \mu \mathrm{L}$ PBS $)$ or PBS alone was injected peri-tumorally 3 times per week, and mice were monitored until the end-point criteria was met. Unexpectedly, tumor volume did not differ between the groups (Fig. 3A), even though OSM has been shown to reduce MDA-MB-231 cell proliferation in vitro (46). The BLI intensities of the tumors from both groups were similar (Fig. 3B), although a few mice in each group had lower BLI intensities due to tumor necrosis.

Mice receiving peri-tumoral OSM showed larger metastatic volumes in both lung and spine compared to mice receiving PBS, as assessed by ex vivo imaging (Fig. 3C, left). Additionally, lungs extracted from the OSM-injected group had luminescence signals that were two orders of magnitude $\left(10^{2}\right)$ higher than the PBS-injected group (Fig. $3 \mathrm{C}$, right). Similarly, spine BLI intensity from OSM-treated mice averaged $2 \times 10^{7}$ photons/s, while control mice had a mean signal of $3 \times 10^{5}$ photons/s.

In patients with advanced and/or inflammatory breast cancer, high numbers of circulating tumor cells (CTCs) have been detected, suggesting a correlation between inflammatory factors and the number of CTCs (47). In our xenograft model, both human MDA-MB-231-Luc2 tumor cells as well as potential CTCs contain multiple copies of human Alu DNA repeat sequences. To assess CTC numbers, DNA was isolated from mouse blood, and the levels of human Alu DNA repeat sequences were determined in the blood by qPCR. In animals that received rhOSM injections, there was a 4-fold increase in the number of CTCs per $100 \mu \mathrm{L}$ of mouse blood compared to animals that did not receive 
OSM (Fig. 3D). Collectively, this suggests that increased paracrine OSM in the tumor microenvironment, increases metastasis to lung and spine while also increasing CTCs. $\underline{\text { Suppression of OSM in syngeneic mouse model reduced lung metastases }}$

To utilize an immunocompetent mouse model, we utilized two highly metastatic 4T1.2 mouse mammary tumor cell lines exhibiting knockdown expression of OSM in a syngeneic, orthotopic model of breast cancer (17). These two independent cell lines (4T1.2-shOSM1 and 4T1.2-shOSM2) were shown by ELISA to secrete a 3- to 12-fold reduction in OSM, respectively, as compared to control 4T1.2-LacZ cells (17). To test the effects of OSM on mammary tumor metastasis in vivo, 1 x $10^{5}$ control 4T1.2-LacZ, 4T1.2-shOSM1, and 4T1.2-shOSM2 cells were injected orthotopically into the mammary fat pads of female Balb/c mice.

The mean number of relative lung metastases was shown to be 10 -fold lower in mice that received 4T1.2-shOSM1 cells and 5-fold lower in mice injected with 4T1.2shOSM2 cells, compared to 4T1.2-LacZ control cells (Fig. 4DA). Histology performed on tissues from mice injected with parental 4T1.2 cells using an anti-mouse OSM antibody showed strong OSM expression in the primary mammary tumor as well as some background expression in the normal breast connective tissue (sFig. 2). Specifically, very high OSM expression is shown at the leading edge of the primary tumor metastasis, in closest proximity to the breast stroma.

Magnetic resonance imaging (MRI) was used to track lung metastases progression in vivo after injection of parental 4T1.2, control 4T1.2-shLacZ, and 4T1.2-shOSM2 cells. Mice were imaged post-injection at days 20-21, days 25-26, and just before sacrifice of animals at days 29-30 (Fig. 4B). For all three cell lines, MRI images showed essentially 
no detectable metastasis at days 20-21. At 25-26 days and 29-30 days; however, readily identifiable metastases were observed in lung images. The number of metastases was reduced by more than $50 \%$ in mice injected with 4T1.2-shOSM2 cells compared to control 4T1.2-shLacZ control cells at 25-26 days and 29-30 days (Fig. 4B and C). The average metastasis volume was also significantly decreased by 50 to $80 \%$ in $4 \mathrm{~T} 1.2$ shOSM2 cells compared to 4T1.2-shLacZ control or parental 4T1.2 cells, respectively (Fig. 4D). Thus, the in vivo MRI imaging confirmed that OSM is a potent inducer of the metastatic cascade that ultimately results in lung metastases originating from a primary mammary tumor. In total, these results suggest that OSM is necessary for spontaneous mammary tumor metastasis to lung in a syngeneic mouse model.

$\underline{\text { Suppression of OSM by shRNA increases survival from spontaneous metastasis via }}$ orthotopic injection but not via intracardiac injection in vivo

We utilized a tumor-resection survival model to mimic surgical removal of the primary tumor in patients and to determine if suppression of tumor-produced OSM limits early metastases. Orthotopic mammary fat pad injections were performed using control 4T1.2-shLacZ cells, 4T1.2-shOSM1, and 4T1.2-shOSM2 cells. Primary tumors were resected when they became palpable at day 14 (Fig. 5A), and mice were monitored until endpoint criteria were met (see Materials and Methods). The mean survival time of the mice that received 4T1.2-shOSM1 and 4T1.2-shOSM2 cell injections significantly increased by 5 and 10 days compared to animals with 4T1.2-shLacZ tumors (Fig. 5A). These results suggest that following primary mammary tumor resection, decreased OSM expression in primary tumor cells leads to increased survival. 
In order to determine if OSM affects post-intravasation aspects of metastasis, we injected the mammary tumor cells directly into the circulatory system via the left ventricle of the heart. There was no statistical difference in the survival time between mice injected intracardially with 4T1.2-shLacZ versus 4T1.2-shOSM2 cells (Fig. 5B). Similarly, there was no statistical difference in lung metastatic burden in the two different tumor types as assessed by qPCR (sFig 3). These results suggest that tumor cell OSM expression has little effect on post-intravasation aspects of metastasis to lung, such as extravasation and metastatic site implantation.

$\underline{\text { CTC number and metastatic burden is reduced in OSM knockout mice compared to wild }}$ type mice

To determine if knocking out OSM in the whole organism affects CTC numbers in the 4T1.2 mouse model, wild type and OSM knockout (KO) Balb/c mice were orthotopically injected with either 4T1.2-shLacZ or 4T1.2-shOSM2 cells. Whole blood was collected at endpoint, red blood cells were lysed, and the remaining white blood cells containing the epithelial CTCs were examined using a Clonogenic assay (Fig. 5C). Blood from OSM KO mice injected with 4T1.2-shOSM2 cells had 15-fold less CTCs and 2.5fold less lung metastases compared to wild type mice injected with control 4T1.2-shLacZ cells (Fig. 5D, left). Additionally, OSM KO mice bearing 4T1.2-shLacZ tumors had 10fold less CTCs and 2.5-fold less lung metastases compared to wild type mice with the same tumor type (Fig 5D, right). These results suggest that microenvironment OSM, independent from the tumor cell secreted OSM, has a large effect on tumor cell dissemination into circulation. Furthermore, this highlights the importance of paracrine OSM in breast cancer progression and metastasis. 
OSM increases pre-intravasation metrics of metastatic capacity in 4T1.2 cells

In order for tumor cells to enter the blood stream as CTCs and subsequently metastasize, it is thought that they must first undergo an EMT, followed by detachment, migration, and intravasation into the circulatory system $(48,49)$. To assess OSM's effect on EMT, detachment, and migration, mesenchymal-like triple negative (ER-, PR-, HER2) $4 \mathrm{~T} 1.2$ mouse mammary cancer cells were treated with OSM $(25 \mathrm{ng} / \mathrm{mL})$ for 24 to 48 hours. As 4T1.2 cells are an aggressive mesenchymal mammary cancer cell type, OSM did not affect their cell morphology nor produce an EMT, (Fig. 6A). On the other hand, OSM significantly increased 4T1.2 mammary tumor cell migration 7-fold by day 3 in a cell migration assay (Fig. 6B) and tumor cell detachment 100-fold by day 8 (Fig. 6C). Our previous studies also demonstrated that OSM increases overall invasive potential in 4T1.2 cells (17). Together, these results suggest that OSM may promote tumor cell dissemination into circulation by increasing cell migration and detachment, which may subsequently increase the number of CTCs.

\section{Discussion}

In this study, we show that OSM, whether acting in a paracrine fashion or produced by breast tumor cells and acting in an autocrine manner, can potentiate preintravasation metastatic events such as migration, detachment, and increased CTCs (Fig. 7). Recent studies suggest that cells from breast ductal carcinoma in situ (DCIS) can actually metastasize prior to their development into malignant invasive ductal carcinoma (IDC), though what triggers this early event has not been well characterized $(50,51)$. Our breast cancer immunohistochemistry studies using tissue microarrays resulted in an intriguing finding; OSM expression is highest in the epithelium of DCIS, as compared to 
IDC, metastatic, or adjacent normal tissue. However, García-Tuñón saw higher levels of breast tissue OSM and OSMR in IDC than in DCIS or normal tissue (13). While there is disagreement in the literature compared to our study in relation to the stage in which the highest level of OSM was seen, there is agreement in so far as higher OSM levels were detected in cancerous tissue versus normal tissue.

In this study, three different TNBC mouse models were utilized, one immunocompetent Balb/c model using syngeneic 4T1.2 cells, and two immunosuppressed athymic xenograft mouse models using either MDA-MB-231-Luc2 or $\mathrm{MDA}^{\mathrm{TO} / \mathrm{OSM}}$ cells. Despite the differences between the systems used in our study, our results were consistent in that suppression of OSM reduced metastasis in Balb/c mice and injection of recombinant hOSM or TET-induced hOSM expression in MDA ${ }^{\mathrm{TO} / \mathrm{OSM}}$ cells increased human breast tumor metastasis in athymic mice. Although, adaptive immunity is stunted in athymic mice due to nonfunctional T-cells, innate immune function is still intact (52). Recent studies indicate that innate immunity plays a primary role in controlling progression of tumor growth and metastatic disease $(53,54)$, and innate immune cells such as macrophages home to hypoxic tumors and promote angiogenesis (19). OSM has recently been shown to increase lung metastatic burden in melanoma by increasing M2 macrophage infiltration (55). Thus, OSM may be promoting inflammatory responses mediated by innate immunity in the tumor microenvironment of both athymic and Balb/c mouse models to promote metastases.

While the traditional cause of mortality in advanced cancer patients is metastasis to vital organs, cachexia has been shown to contribute in up to $50 \%$ of cancer patient deaths $(56,57)$. Significant weight loss as a consequence of fat loss and muscle wasting, 
indicative of cachexia, was seen in our TET-induced OSM in MDA ${ }^{\mathrm{TO} / O S M}$ xenograft mouse model. Other studies have shown that high levels of various inflammatory cytokines such as IL-6, and tumor necrosis factor alpha (TNF $\alpha$ ) potentiate loss of adipose tissue and muscle wasting $(58,59)$. This suggests that OSM may be yet another cytokine that could exacerbate cachexia in breast cancer patients. Furthermore, since cytokines modulate the immune system, it's very probable that cancer cachexia could be related to maladaptive immune responses (60).

Platelets, as an adjunct to their classical role in thrombosis, are also operant in mediating inflammation and immune response $(61,62)$. Interestingly, we found in the $\mathrm{MDA}^{\mathrm{TO} / O S M}$ mouse model that higher OSM levels were correlated with increased platelet counts and reduced animal survival. Previous studies in breast cancer patients have shown that elevated platelet counts were associated with poor prognosis and reduced disease-free survival (63). Platelets have also been implicated in the promotion of metastasis by acting as a reservoir for factors that induce invasion and function to protect CTCs from the immune system $(64,65)$. In our study, increased viable CTCs were detected in our mouse models when higher levels of OSM were present, which may have been due, in part, to the elevated levels of platelets in circulation.

The ability of tumor cells to intravasate into the circulation directly correlates with CTC numbers from the corresponding tumor (66). Increased CTC numbers have been linked clinically to enhanced metastatic burden in patients and a reduced 5-year survival rate (67). As tumors may shed early during cancer development (50), the detection of CTCs would be an important tool in the clinic to assess the metastatic capacity of a tumor, even as early as in precancerous DCIS. Typically, CTCs are detected 
using cancer epithelial markers such as cytokeratin (CK) 18/19 (68). However, highly aggressive tumor cells that have already undergone an EMT, and thus lost their epithelial markers, may evade detection $(69,70)$. Other possibly viable markers for CTC detection include, epithelial cell adhesion molecule (EpCAM) or human mammoglobin A (hMAM) (68). For our studies, the highly aggressive mesenchymal-like MDA-MB-231 cells show negative or low expression for each of these markers, making conventional CTC detection unfeasible $(69,71)$. Thus, we employed multiple techniques that are markerindependent, such as the Colony forming assay or a PCR assay targeting human Alu sequences in human breast tumor cells growing in a mouse. In our studies, suppression of tumor-produced OSM or the absence of OSM in OSM KO mice resulted in reduced numbers of CTCs, while injection of recombinant OSM increased CTCs. Interestingly, there was no significant difference in the number of CTCs +/-TET in the MDA ${ }^{\mathrm{TO} / O S M}$ mouse metastasis model (data not shown). This suggests that paracrine OSM may be more important than autocrine-produced OSM for CTC development. Indeed, in our OSM KO mouse model, where there is less paracrine OSM, total CTC numbers and lung metastatic burden were significantly reduced compared to WT mice.

To assess some of the early aspects of metastases that could lead to generation of CTCs in vitro, tumor cell EMT, migration, and detachment were studied in the highly aggressive 4T1.2 tumor model. The 4T1.2 mammary tumor cells, which are considered analogues of high-grade human TNBC, have already undergone EMT and OSM did not cause additional EMT-like effects (72). However, our results show that OSM does increase cell migration, detachment, and invasion in the 4T1.2 cells (17), supporting the idea that OSM operates in the pre-intravasation steps of metastasis. Other OSM-related 
factors such as transforming growth factor beta (TGF $\beta$ ), IL-6, and interleukin-8 (IL-8) may promote CTCs by increasing tumor cell invasion, detachment, and epithelial to mesenchymal transition $(\mathrm{EMT})(73,74)$. Furthermore, these effects may be amplified in vivo, as OSM possesses a proclivity to accumulate in the acidic ECM of the tumor microenvironment (21).

Based on our findings, OSM may potentiate the pre-intravasation aspects of the metastatic cascade to increase metastasis to the lung, and bone (17). This is evidenced by the fact that intracardiac injection of 4T1.2 cells with reduced OSM (4T1.2-shOSM2) into mice, which bypasses intravasation step of the metastatic cascade, did not result in increased survival compared to control cells (4T1.2-shLacZ). Therefore, it is highly probable that OSM functions before intravasation during cancer progression, but does not appear to affect CTC survival, tumor cell extravasation, and secondary tumor growth.

\section{Conclusions}

The results from this study suggest that OSM increases lung metastases and CTC numbers by acting on the early stages of metastasis. This provides a rationale for the administration of anti-OSM therapeutics before tumor resection at the earlier steps of the disease with the potential to improve overall breast cancer patient survival.

\section{Declarations}

Breast tissue that was analyzed for this publication was obtained from paraffin block archives at the Department of Pathology, Mercy Medical Center, Nampa, ID and deidentified, as per their Institutional Review Board guidelines. 
All animal experiments were approved by and performed in accordance with the animal guidelines of the Boise Veterans Affairs Medical Center (\#JOR0010-2) and the Boise State University (\#006-AC16-007) Institutional Animal Care and Use Committees. 
Chapter Three: Figures

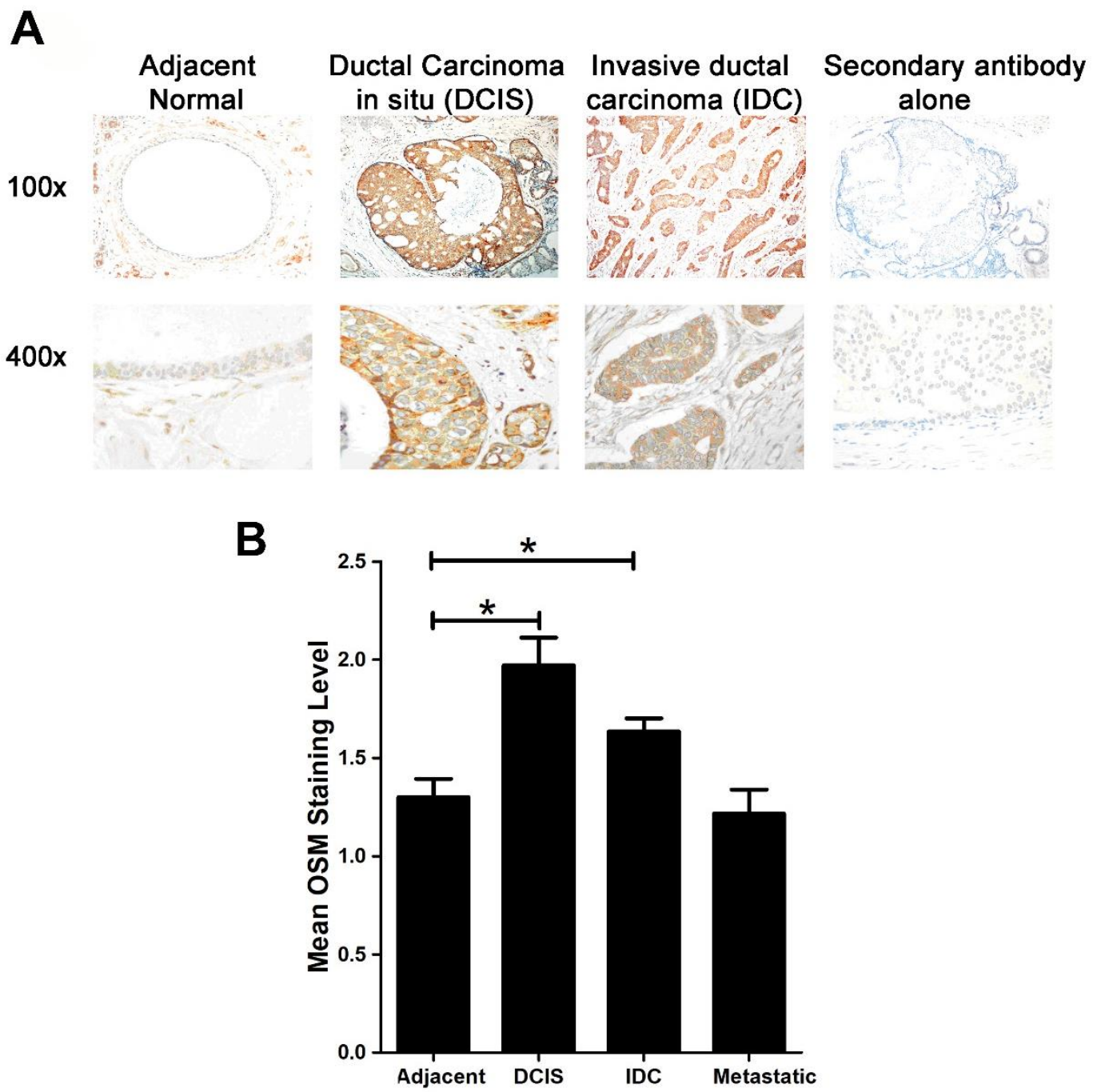

Figure 3.1. OSM is highly expressed in DCIS and IDC 
Figure 3.1. OSM is highly expressed in DCIS and IDC.

A, To detect the presence of OSM in breast cancer tissue, histological microarrays from 72 breast cancer patients were stained with human OSM antibody by immunohistochemistry. Twelve patients had ductal carcinoma in situ (DCIS), 54 patients had non-metastatic invasive ductal carcinoma (IDC), and 16 patients had IDC with metastasis to lymph nodes (Table 1). Results show that normal adjacent tissue does not express much OSM, but OSM is highly expressed in DCIS and IDC. Secondary antibody alone does not produce any background signals. B, Intensity quantification of OSM stained tissues. Mean staining intensity for DCIS (2.00) and IDC (1.66) tissues are significantly higher than normal adjacent tissue (1.33) and metastatic tissue (1.24). There is no statistical difference between normal and metastatic tissue. Data expressed as mean +/- SEM *p<0.05, two-tailed t-test. 


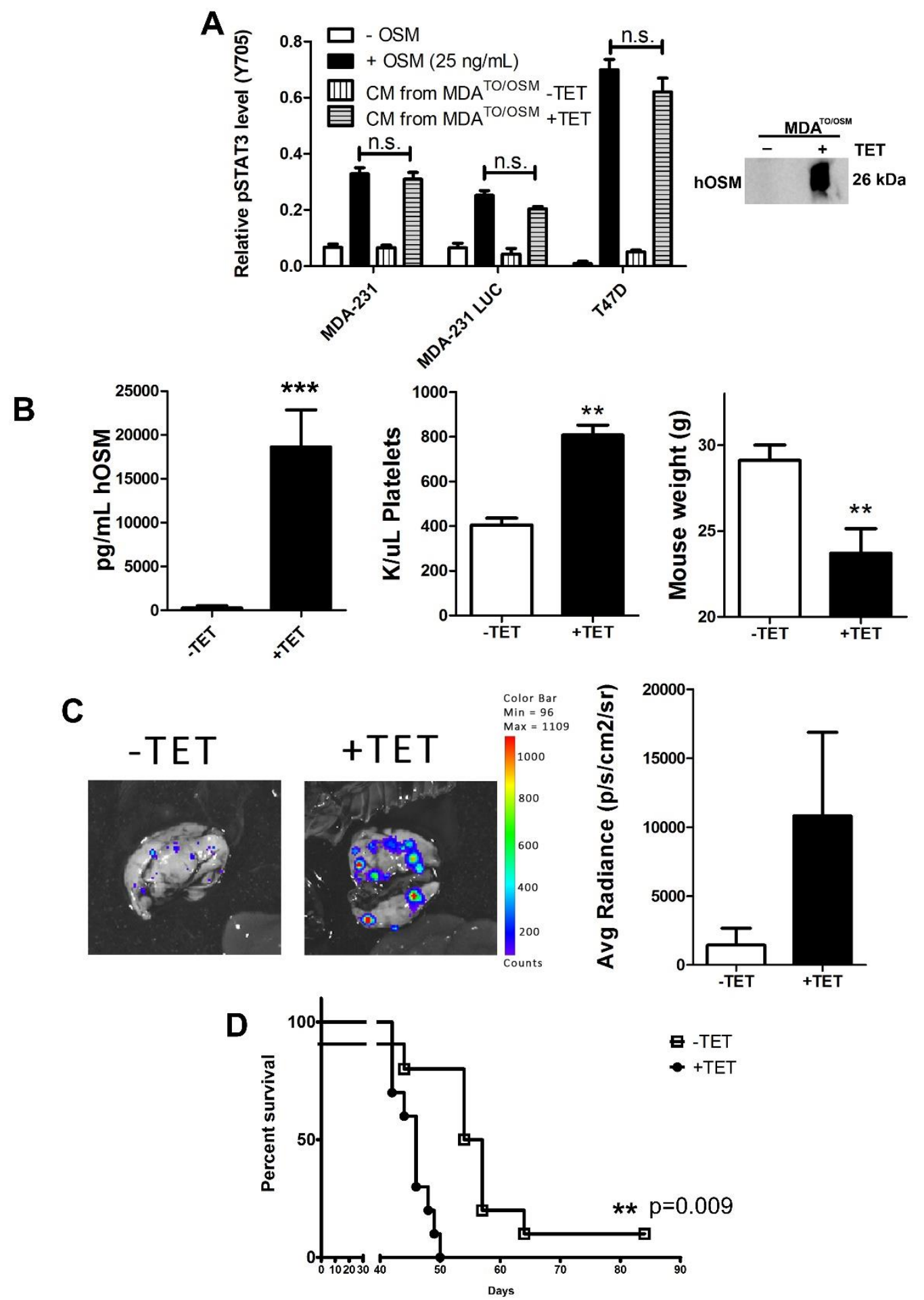

Figure 3.2. $\mathrm{MDA}^{\mathrm{TO} / \mathrm{OSM}}$ tumors increase metastasis and decrease survival 
Figure 3.2: MDATO/OSM tumors increase metastasis and decrease survival.

A, $\mathrm{MDA}^{\mathrm{TO} / \mathrm{OSM}}$ human breast cancer cells were treated with or without TET and the resultant CM from the treated cells were applied to parental MDA-MB-231, MDAMB-231-LUC, and T47D cells. The activity of OSM accumulated in the CM was compared to commercially obtained rhOSM $(25 \mathrm{ng} / \mathrm{mL})$. There is no significant difference between OSM produced by MDA ${ }^{\mathrm{TO} / O S M}$ versus rhOSM in relation to its ability to induce pSTAT3. Inset, Western blot analysis depicting that CM produced by $\mathrm{MDA}^{\mathrm{TO} / \mathrm{OSM}}$ cells stimulated with TET contains OSM. B, Left, Animals with MDA ${ }^{\mathrm{TO} / O S M}$ tumors were given drinking water with or without TET and the whole blood was collected at experimental endpoint. After allowing the blood to clot and serum separated by centrifugation, the resultant serum OSM levels were measured by ELISA, and animals with $\mathrm{MDA}^{\mathrm{TO} / \mathrm{OSM}}$ tumors with drinking water containing TET have 67-fold higher OSM levels. B Center, Platelet counts are higher in MDA ${ }^{\mathrm{TO} / O \mathrm{SM}}$ tumor bearing mice $+\mathrm{TET}$ compared to -TET mice. B Right, MDA ${ }^{\mathrm{TO} / O S \mathrm{M}}$ tumor bearing mice + TET have lower bodyweight compared to mice -TET. C, Animals with MDA ${ }^{\mathrm{TO} / O \mathrm{SM}}$ tumors were given drinking water containing TET for 1 week and their lung metastatic levels were assessed by ex vivo bioluminescent imaging. C Left, Representative image of ex vivo bioluminescent image. C Right, Average radiance analysis of the ex vivo bioluminescent imaging in photons per second per $\mathrm{cm}^{2}$ per square radian $(\mathrm{p} / \mathrm{s} / \mathrm{cm} 2 / \mathrm{sr})$. Animals with $\mathrm{MDA}^{\mathrm{TO} / O S \mathrm{M}}$ tumor + TET have a 5-fold higher bioluminescent radiance compared to TET mice. ( - TET $n=3,+$ TET $n=6$ ) Data expressed as mean \pm SEM. D, Kaplan-Meier survival curve for mice with MDA ${ }^{\mathrm{TO} / O S M}$ tumors $+/$ - TET. Mice that did not receive TET have on average, 11 days longer survival $(-\mathrm{TET} n=9,+$ TET $n=10) * * * \mathrm{p}<0.001 \log$-rank 
test. Data expressed as mean $+/-$ SEM $* \mathrm{p}<0.05, * * \mathrm{p}<0.01$, $* * * \mathrm{p}<0.001$, two-tailed $\mathrm{t}$-test or one-way ANOVA with Tukey's post-test where appropriate. 


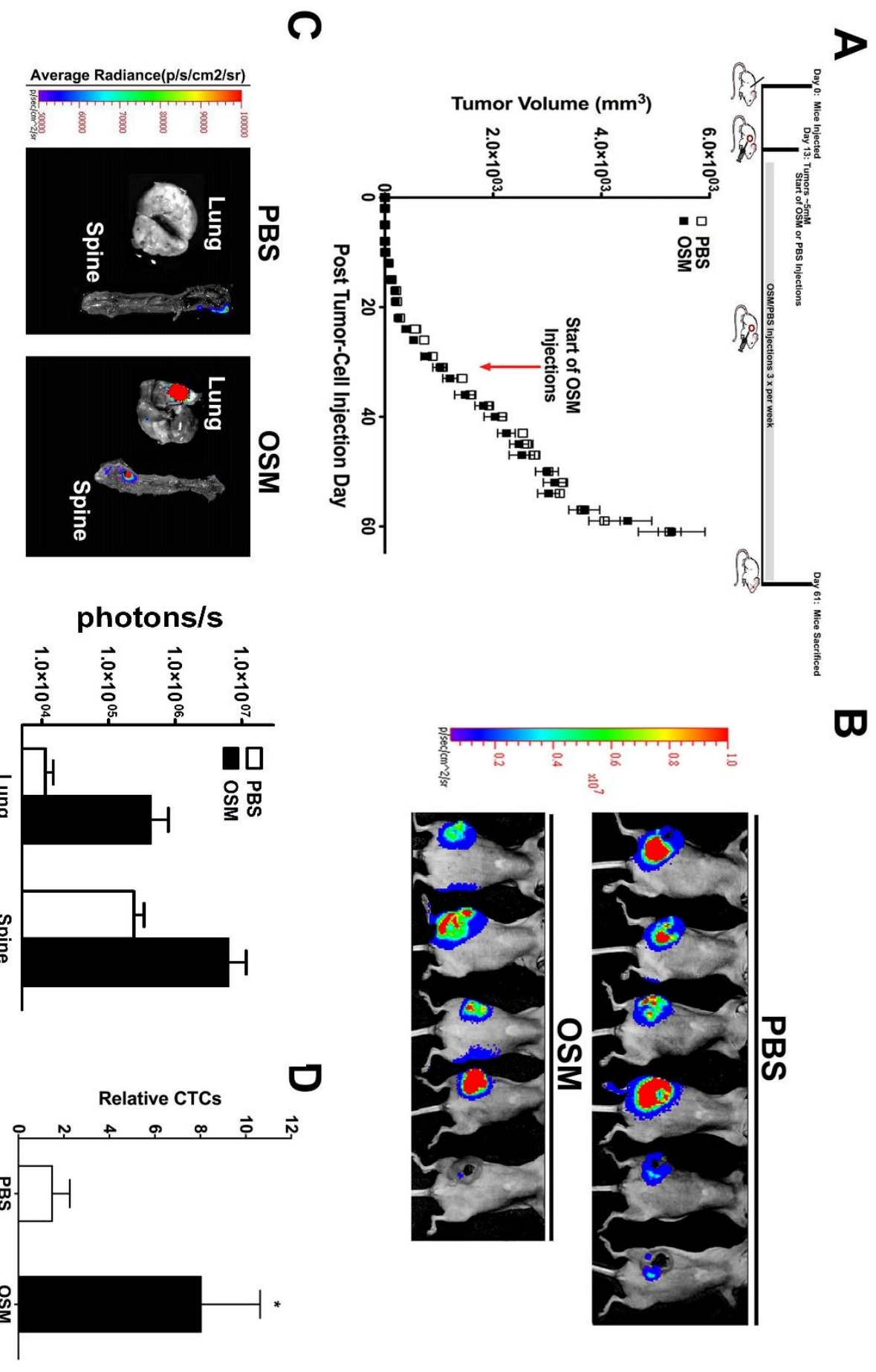

Figure 3.3. Peri-tumoral OSM injections into mice with MDA-MB-231-D3H2LN tumors promotes the development of metastases and CTCs 
Figure 3.3. Peri-tumoral OSM injections into mice with MDA-MB-231 D3H2LN tumors promote the development of metastases and CTCs. A, The timeline shows orthotopic MDA-MB-231 D3H2LN human breast tumor cell injection at day 0, peri-tumoral OSM or PBS injections beginning $3 \mathrm{X}$ per week at day 13 , and final day of sacrifice of both groups at day 61. Average tumor volume $\left(\mathrm{mm}^{3}\right)$ does not differ between the peritumorally injected OSM and PBS control groups. B, Representative images of PBS and OSM injected tumor-bearing mice imaged ventrally by BLI. There is no statistical difference between the groups and recapitulates results seen in A. C left, Representative ex vivo BLI of lungs and spine from mice bearing MDA-MB-231 D3H2LN luc2 tumors, which were treated with peri-tumoral injections of PBS or OSM. C right, Ex vivo BLI intensities were quantified in the lung and spine. Lungs from mice receiving peri-tumoral OSM injections show a 37.9-fold higher BLI intensity as compared to PBS injections, and spines from mice injected with OSM also show an approximately 25.9-fold increase over mice with PBS injections. Data expressed as photon/s (mean $\pm \mathrm{SEM}$; $=5-6$ ). D, Human CTCs containing human Alu DNA were detected from mouse blood by qPCR. In animals that received OSM injections, there is a 4-fold increase in the number of CTCs compared to controls. Data expressed as mean $+/$ - SEM * $\mathrm{p}<0.05$, two-tailed t-test. 


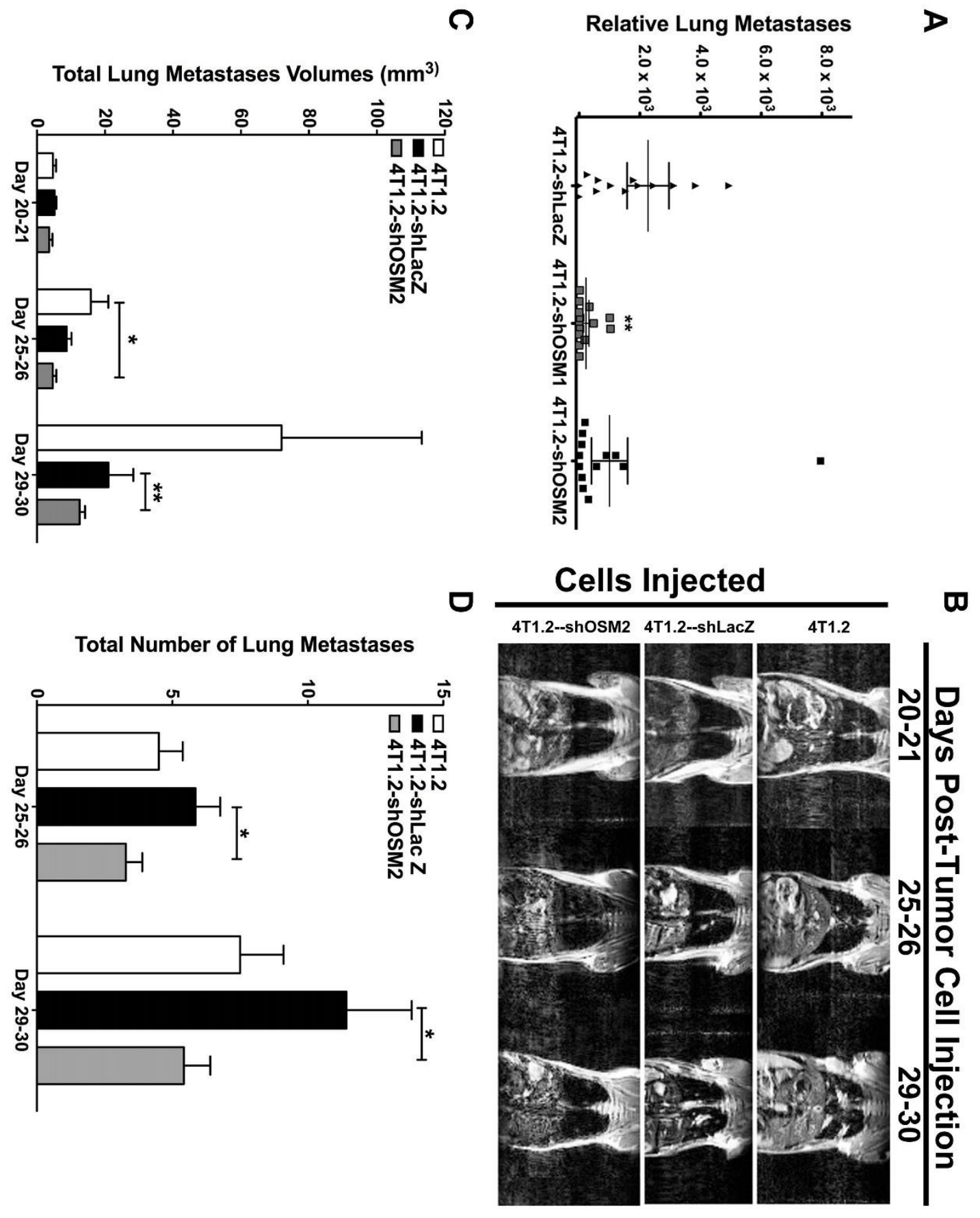

Figure 3.4. Reduced OSM expression results in fewer spontaneous lung metastases and lower total volume of lung metastases by MRI 
Figure 3.4. Reduced OSM expression results in fewer spontaneous lung metastases and lower total volume of lung metastases by MRI.

A, Lung metastatic burden was quantified by qPCR. Mice bearing mouse mammary 4T1.2-shOSM1 or 4T1.2-shOSM2 tumors have less metastasis to lung compared to mice with 4T1.2-shLacZ tumors. B, Mice bearing 4T1.2-shOSM2 tumors have less metastatic lesions in the lung as detected by MRI at the endpoint of the experiment compared to mice bearing parental 4T1.2 or control 4T1.2-shLacZ tumors. C, MRI quantification of lung metastatic volume, and $\mathbf{D}$, total number of lung metastases, shows significantly higher volume and number of lung metastases in the 4T1.2 or 4T1.2shLacZ injected mice as compared to the 4T1.2-shOSM2 injected mice. (4T1.2, n=6; 4T1.2-shLacZ, $n=7 ;$ TT1.2-shOSM2, $n=7)$. Data expressed as mean $\pm \mathrm{SEM}$, ${ }^{*} \mathrm{p}<0.05$, **p $<0.01$, one-way ANOVA with Tukey's post-test. 


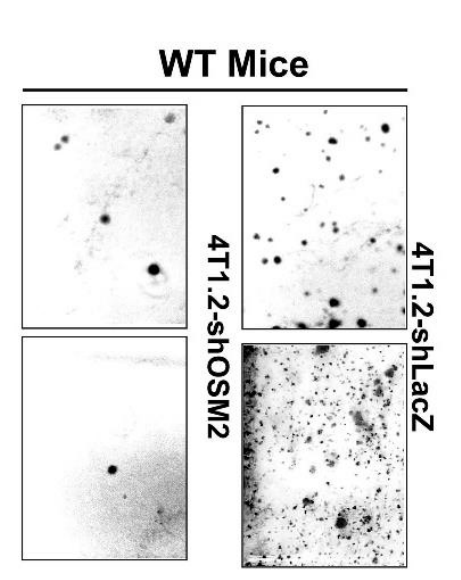

$\Omega$
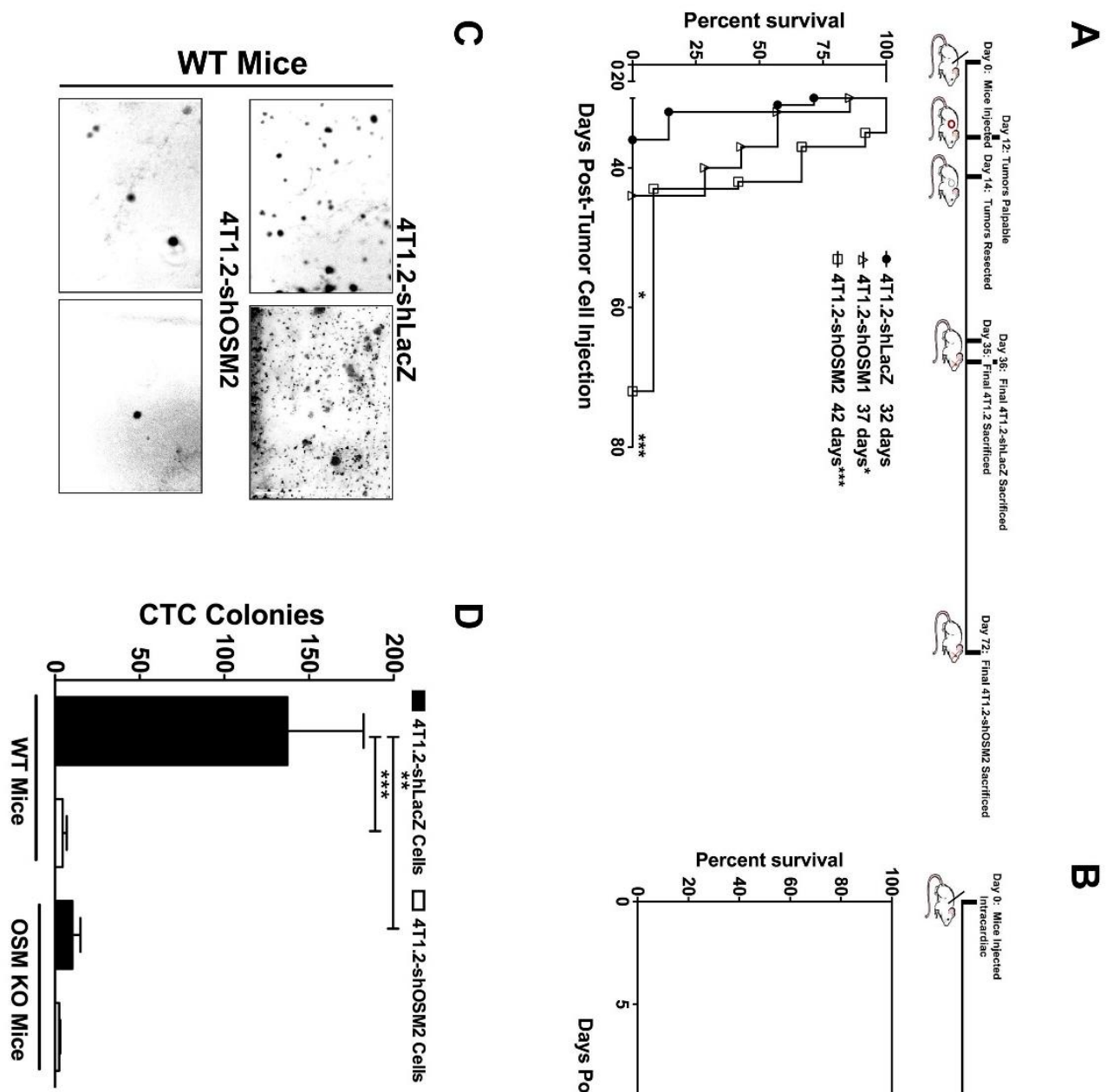

No. of Lung Mets
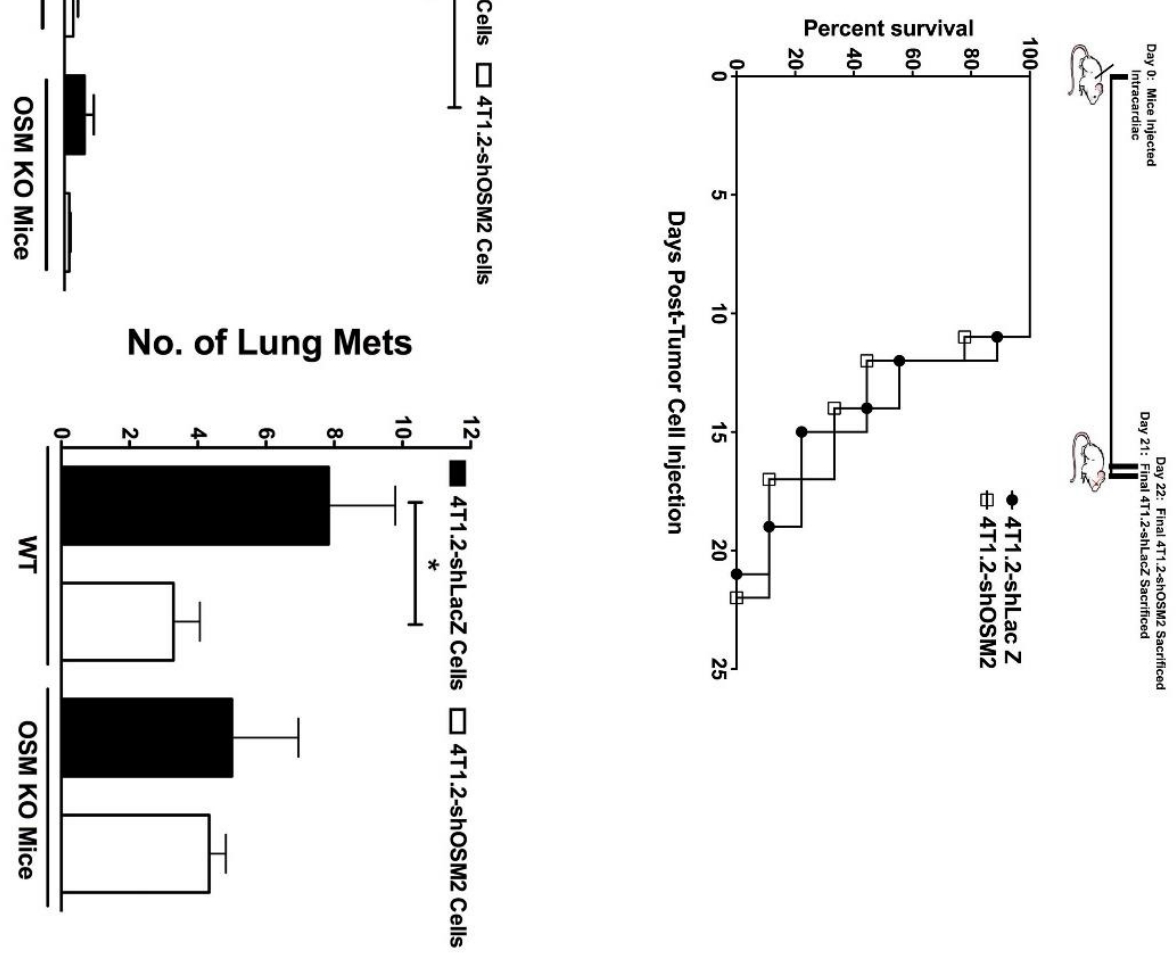

$\boldsymbol{\omega}$

Figure 3.5. Reduced tumor cell OSM expression increases survival in a 4T1.2shOSM mouse model of tumor resection 
Figure 3.5. Reduced tumor cell OSM expression increases survival in a 4T1.2-shOSM mouse model of tumor resection.

A. The timeline shows orthotopic mouse mammary tumor cell injection at day 0 , resection at day 14, and final day of sacrifice per group (ranging from 35 to 72 days). Kaplan-Meier survival analysis following tumor resection shows that mice bearing 4T1.2-shOSM1 or 4T1.2-shOSM2 tumors have significantly increased survival compared to mice with control 4T1.2-shLacZ tumors. *p<0.05, log-rank test $\mathbf{B}$, Timeline shows intracardiac mammary tumor cell injection at day 0 and final day of sacrifice (day 21 to 22). Kaplan-Meier survival analysis shows no difference in survival between mice injected with control 4T1.2-shLacZ versus 4T1.2-shOSM2 cells. C, Blood was collected from wild type and OSM KO animals with 4T1.2-shOSM2 or control tumors and CTC counts were assessed via a Colony forming assay. Representative image depicts higher numbers of colonies that formed from the blood collected from wild type mice with control tumors. D Left, Quantification of the colony formation assay show that wild type animals bearing 4T1.2-shOSM2 tumors have 15 -fold lower number of CTCs compared to the animals bearing control 4T1.2-shLacZ tumors. Furthermore, OSM KO mice with 4T1.2-shLacZ tumors have 10-fold less CTCs compared to wild type mice bearing the same cells. D Right, Wild type mice bearing 4T1.2-shOSM2 tumors have 2.5-fold lower number of lung metastases compared to mice with control 4T1.2-shLacZ tumors. OSM KO mice bearing 4T1.2-shLacZ or 4T1.2-shOSM2 tumors have 2- to 2.5 -fold less lung metastases compared to wild type mice. (4T1.2-shLacZ, n=8-9; 4T1.2-shOSM1, n=7; 4T1.2-shOSM2, $\mathrm{n}=9-12$ ) Data expressed as mean \pm SEM. $* \mathrm{P}<0.05$, $* * * \mathrm{P}<0.001$, oneway ANOVA with Tukey's post-test. 


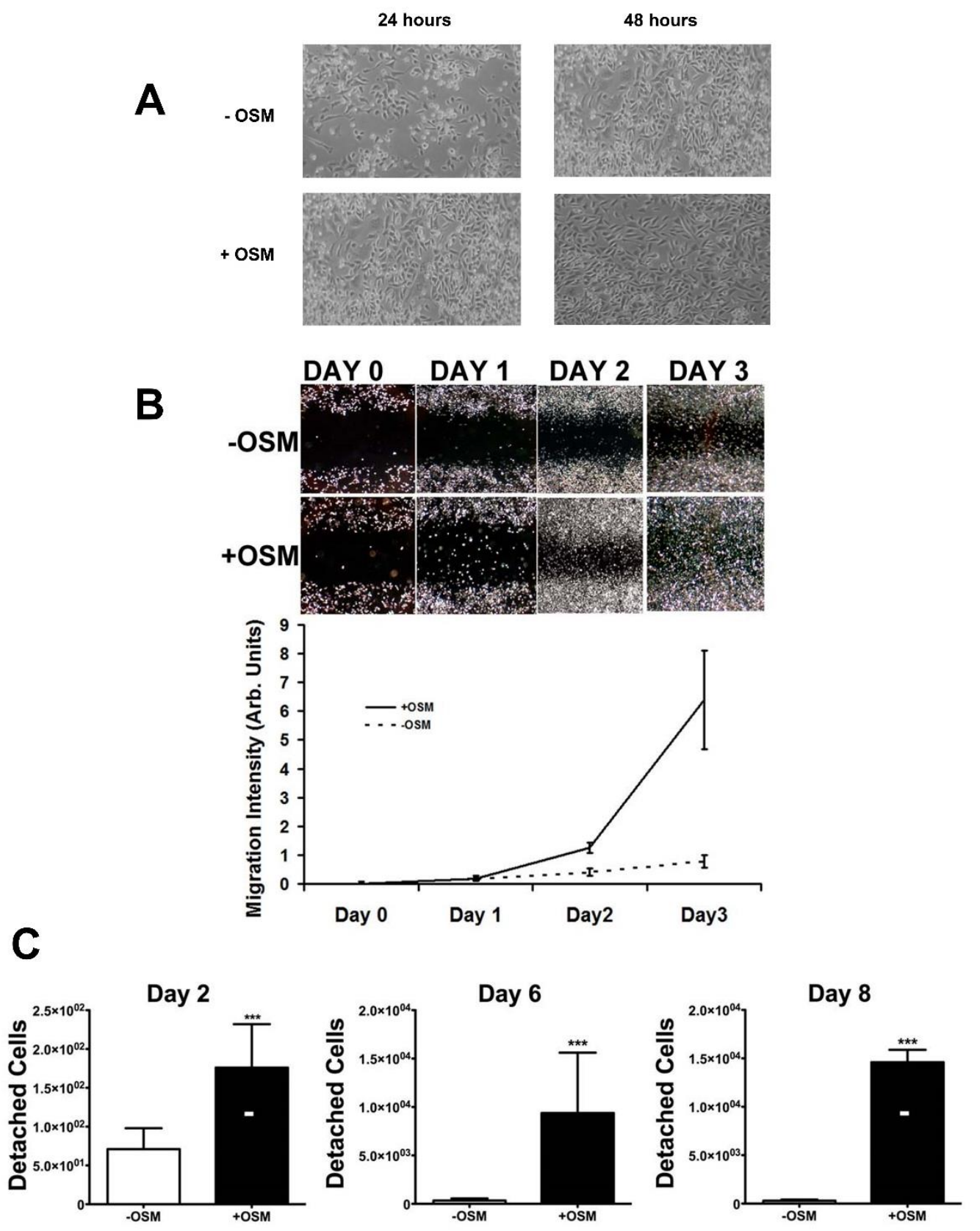

Figure 3.6. OSM promotes 4T1.2 cell detachment and migration 
Figure 3.6. OSM promotes 4T1.2 cell detachment and migration.

A, 4T1.2 mouse mammary cancer cells were plated and treated with rmOSM (25 $\mathrm{ng} / \mathrm{mL}$ ) for 24 and 48 hours, and no morphological changes indicative of EMT are detected. B, 4T1.2 cells were grown to $80 \%$ confluency and a scratch was made. Cells treated with OSM have higher levels of migration compared to untreated controls (7-fold by day 3). C, A detachment assay was performed on $4 \mathrm{~T} 1.2$ cells, and the number of detached cells was quantified. Cells treated with OSM have significantly higher numbers of detached cells (100-fold at day 8). Data expressed as mean \pm SEM. ${ }^{*} \mathrm{p}<0.05$, $* * * \mathrm{p}<0.001$ two-tailed student's t-test. 


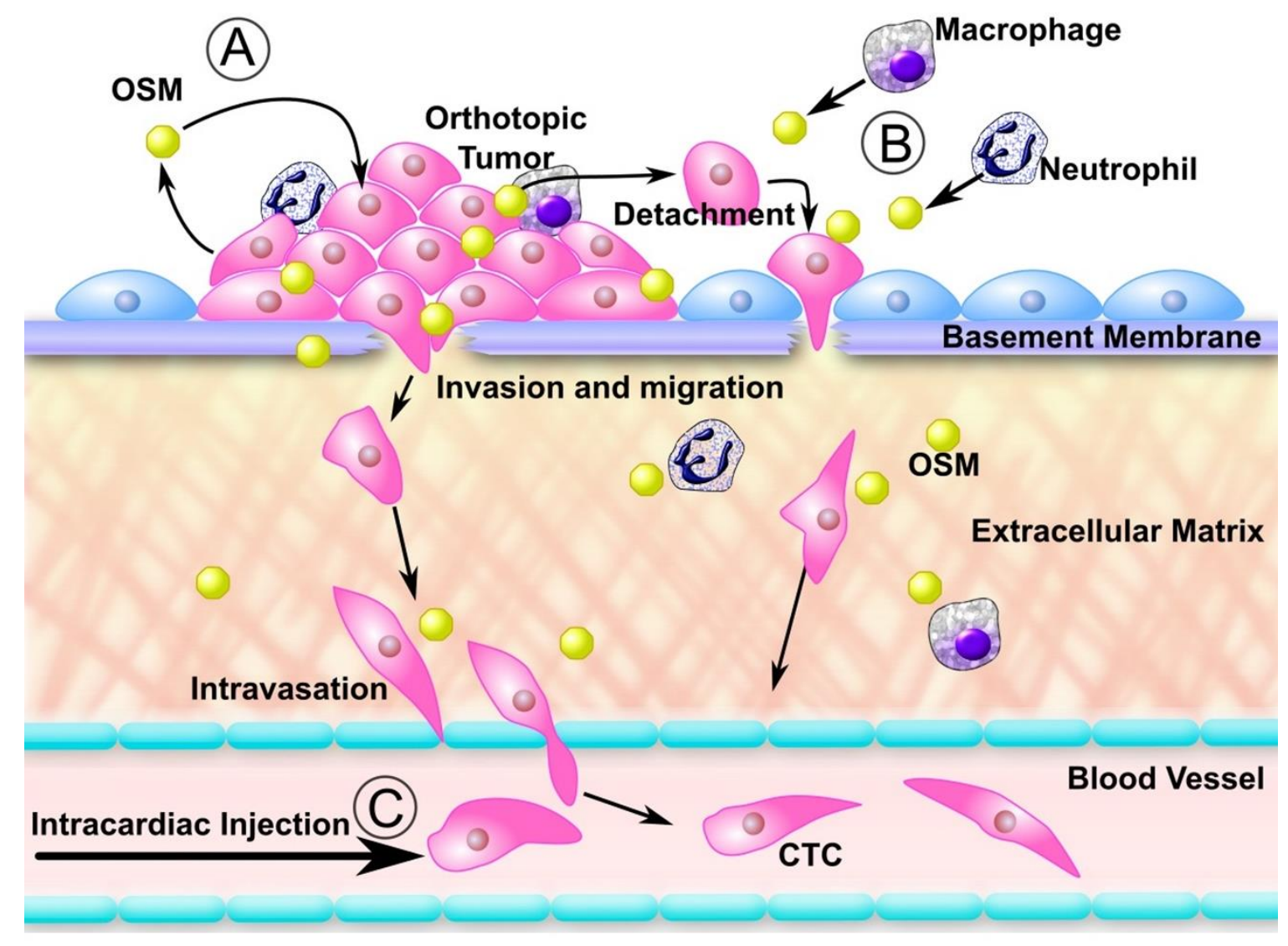

Figure 3.7. Model of OSM-mediated metastasis

Figure 3.7. Model of OSM-mediated metastasis.

OSM is produced in an autocrine fashion by tumor cells $\mathbf{A}$, and by tumorassociated macrophages and neutrophils for paracrine signaling B. OSM promotes preintravasation effects such as tumor cell detachment and migration that can drive tumor cell intravasation into circulation to develop CTCs and eventual metastasis. C, When tumor cells are injected directly into the circulatory system, they bypass the multi-step pre-intravasation aspects of metastases, and our data suggest that OSM has little effect on their extravasation and colonization at a secondary site. 
Table 3.1 Comparison of OSM expression in the ductal epithelial cells of different stages of ductal carcinoma of the breast (DCIS, IDC, and metastatic tissues) and adjacent normal breast tissues.

Mean expression levels are statistically significantly different among the four stages.

\begin{tabular}{|l|l|l|l|l|}
\hline Stage & $\begin{array}{l}\text { No. of } \\
\text { patients } \\
\text { (Total no. of } \\
\text { cores) }\end{array}$ & $\begin{array}{l}\text { Mean } \\
\text { OSM } \\
\text { staining }\end{array}$ & $\begin{array}{l}\text { 95\% Confidence } \\
\text { Limits }\end{array}$ & $\begin{array}{l}\text { Pairwise comparison } \\
\text { of stage means }\end{array}$ \\
\hline Adjacent & $50(83)$ & 1.33 & $(1.15,1.50)$ & A \\
Normal DCIS & $12(18)$ & 2.00 & $(1.71,2.30)$ & B \\
IDC & $72(188)$ & 1.66 & $(1.55,1.77)$ & B \\
Metastatic & $16(29)$ & 1.24 & $(1.02,1.46)$ & A \\
& \\
\hline
\end{tabular}




\section{CHAPTER THREE: SUPPLEMENTAL FIGURES}

A

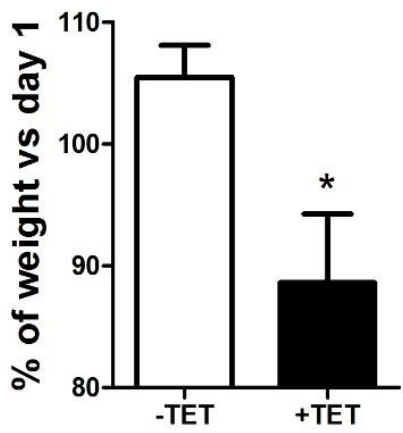

C

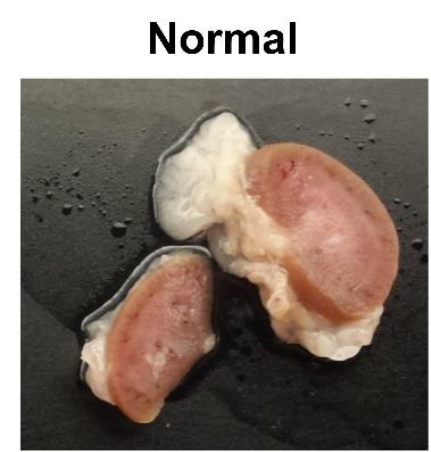

D
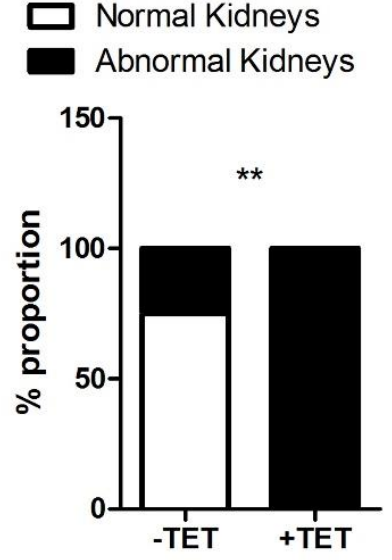

B

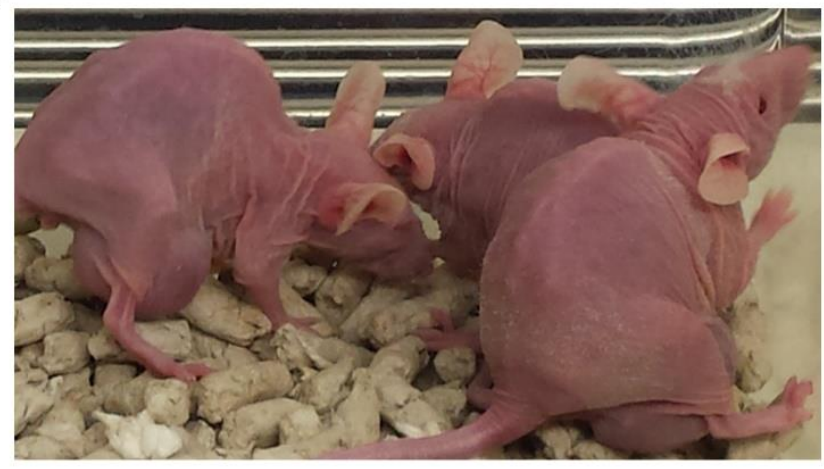

Abnormal (Hypoperfused)

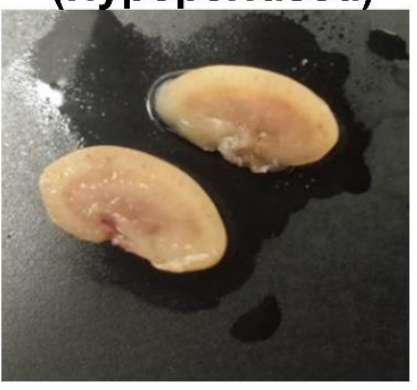

E

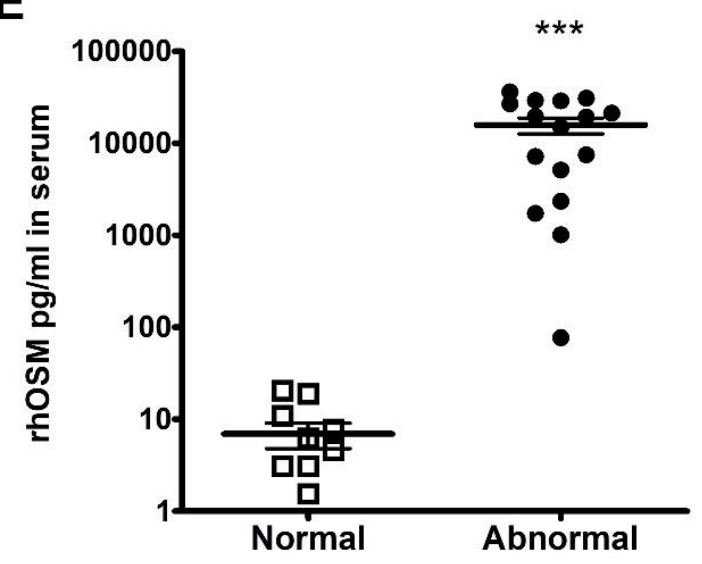

Figure 3.S1. Deterioration of body condition in $\mathrm{MDA}^{\mathrm{TO} / \mathrm{OSM}}$ tumor bearing mice with TET 
Figure 3.S1. Deterioration of body condition in MDATO/OSM tumor bearing mice treated with TET.

A, $\mathrm{MDA}^{\mathrm{TO} / \mathrm{OSM}}$ tumor bearing mice treated with tetracycline (+TET) lost on average $11.4 \%$ of their body weight during TET treatment, compared to -TET mice, which gained on average of $5.5 \%$ of their body weight over the same time period. B, Representative image of mice with MDA ${ }^{\mathrm{TO} / \mathrm{OSM}}$ tumors + TET shows prominent spinal column, muscle wasting, and lack of visible adipose tissue. C, Gross morphology of normal (left) and abnormal kidneys (right). Normal kidneys have a distinct border between the medulla and the cortex, with the cortex shown as a darker pink/red color, and the medulla shown as a lighter pink color. This indicates that normal blood perfusion was taking place. Abnormal kidneys were both pale and hypoperfused (middle), or damaged (right) with no clear distinction between the cortex and the medulla. D, One hundred percent of mice in the +TET group have abnormal kidney morphologies, while only $25 \%$ of the mice in the -TET group have abnormal kidneys. ${ }^{* *} p<0.01$ fisher's exact test $\mathbf{E}$, Serum from mice with abnormal kidneys have a statistically higher level of OSM compared to serum from mice with normal kidneys. Data expressed as mean \pm SEM. ${ }^{*} \mathrm{p}<0.05, * * \mathrm{p}<0.01,{ }^{* * *} \mathrm{p}<0.001$ two-tailed student's t-test. 


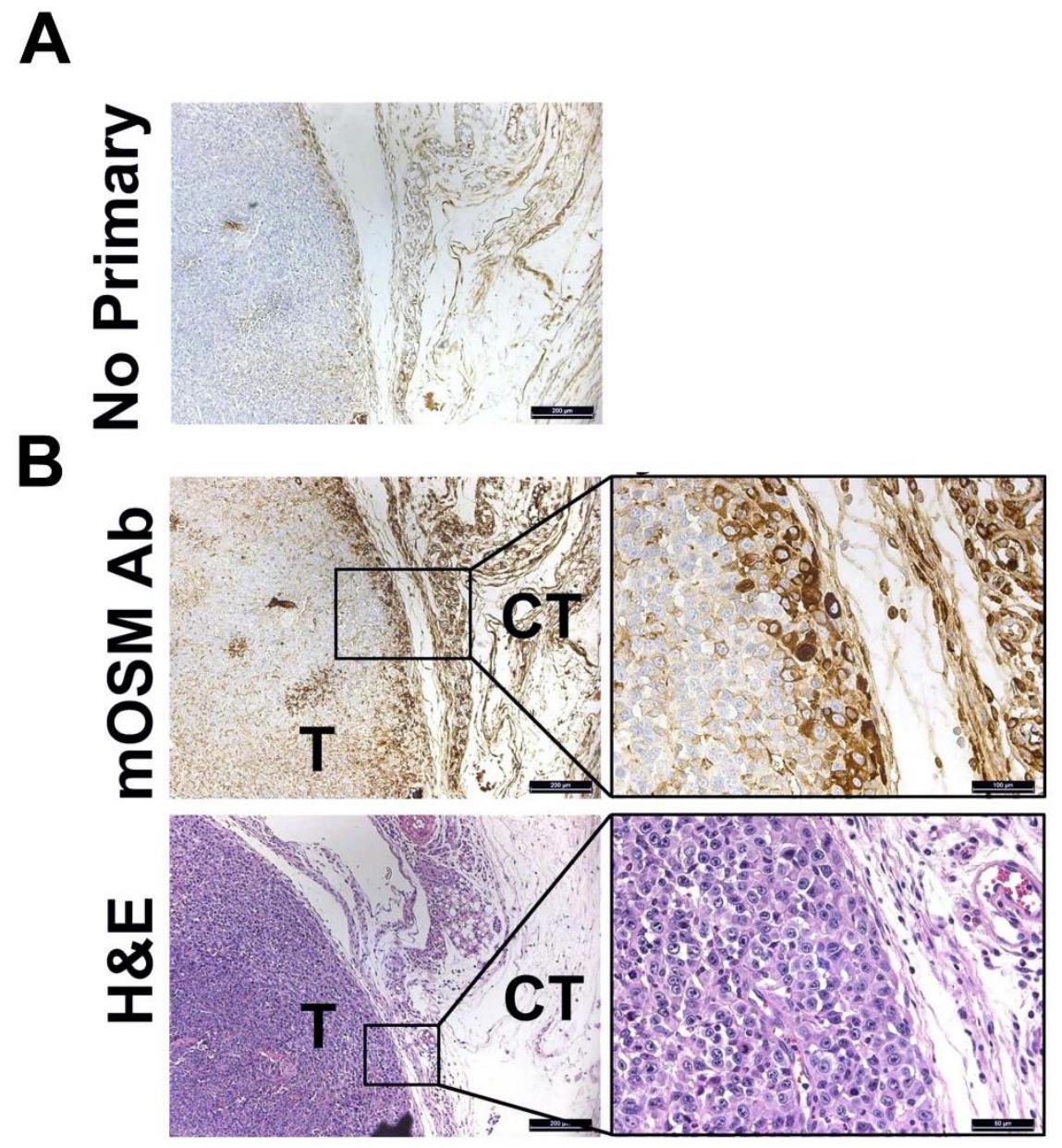

Figure 3.S2. OSM is highly expressed in orthotopic 4T1.2 primary mammary tumors in female Balb/c mice 
Figure 3.S2. OSM is highly expressed in orthotopic 4T1.2 primary mammary tumors in female Balb/c mice.

Histology using H\&E confirms the presence of a large, primary mammary tumor (T) 32 days after 4T1.2 mouse mammary tumor cell injection into the $4^{\text {th }}$ mammary fat pad of female Balb/c mice. High OSM expression is seen in the tumor, as is background expression in the normal breast connective tissue (CT). OSM expression is shown to be highest in the invasive edge of the tumor $(\mathrm{T})$ closest to the normal breast connective tissue (CT). Control slides with no primary OSM antibody show low background staining. 


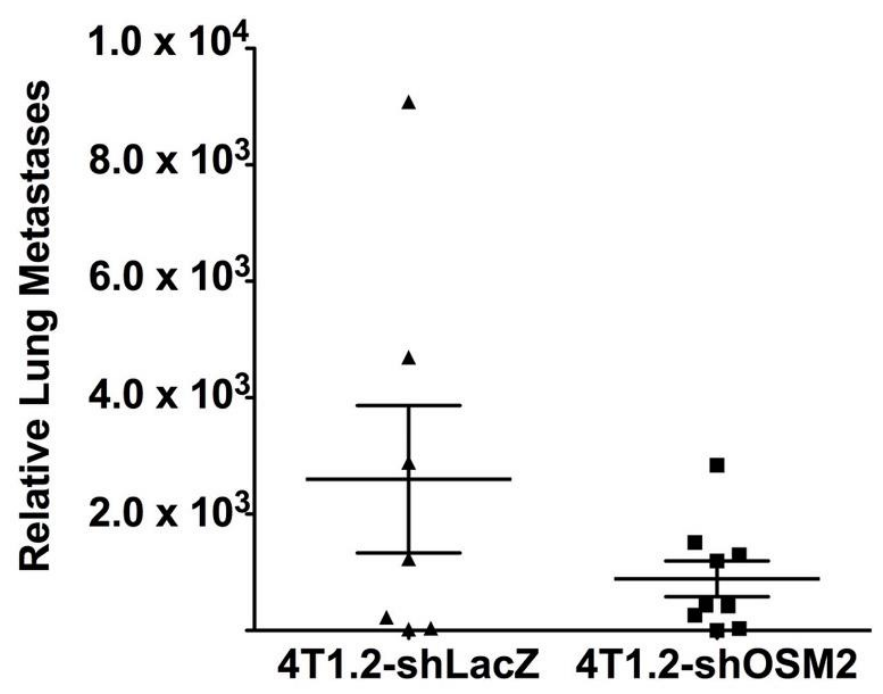

Figure 3.S3. qPCR analysis of lung metastases after intracardiac injections

Figure 3.S3. qPCR analysis of lung metastases after intracardiac injections.

4T1.2-shLacZ cells and 4T1.2-shOSM2 cells were injected through intracardiac injection, and qPCR analysis of the lung metastases indicate that the difference between the groups was not significant. 


\section{Supplemental Materials and Methods}

Complete details for tissue microarrays.

Breast tissue was obtained from paraffin block archives at the Department of Pathology, Mercy Medical Center, Nampa, ID and removed of patient identity as per IRB guidelines. Three tissue microarrays (TMA) of $1 \mathrm{~mm}$ thickness and totaling 72 patients were made using a Quick-Ray, an instrument used for boring tissue from a paraffin block (Woo-Ri Medic, Kent, WA). The TMAs were built in Dr. William Fyffe's lab at Northwest Nazarene University, Nampa, ID. Two blocks consisted of tissues from 54 breast cancer patients (32 adjacent normal, 9 DCIS patients, 54 IDC) without metastasis and included three primary tumor cores and one adjacent normal core for each case. The third block included samples from a total of 18 breast cancer patients (18 adjacent normal, 3 DCIS, 18 IDC and 16 metastatic) with lymph node metastasis and contained three primary tumor cores, two metastatic cores and one adjacent normal tissue core per case. The TMAs included a row of control tissues including spleen, lung, placenta, salivary gland, liver and brain. Spleen and salivary gland served as positive controls for OSM staining.

Immunohistochemistry: The TMAs were stained for oncostatin M using the Histostain Kit (Cat \#95-9843; Invitrogen, Carlsbad, CA) per manufacturer's instructions. The TMAs were deparafinized using Histosol (National Diagnostics, Atlanta, GA) and stained overnight with 1:400 dilution of rabbit anti-human OSM primary antibody (Cat \#sc-129; Santa Cruz Biotechnology, Santa Cruz, CA) and 1 hour with 1:1000 goat-anti rabbit IgG-AP secondary antibody. TMAs stained with secondary antibody alone served as the negative control, and spleen and salivary gland served as positive controls for 
OSM staining. The specificity of the a-OSM antibody was tested by treating tissue sections with 10 times the amount of OSM blocking peptide (Santa Cruz

Biotechnologies, Santa Cruz, CA) and incubating overnight at $4{ }^{0} \mathrm{C}$. The mixture was then diluted to the required concentration and immunohistochemistry was performed as above.

The TMAs were analyzed for OSM expression by an experienced pathologist, Dr. Joseph Kronz, at Mercy Medical Center, Nampa, ID. The intensity of OSM staining in the ductal epithelium was graded as follows: $0=$ No staining; 1=Light staining; 2=Medium staining; 3=Dark staining. In order to confirm reproducibility of the results, the pathologist, Dr. Joseph Kronz, reread 10 TMA cores that were chosen at random, and his observations were consistent with the previous results. In cases where a single core had both cancerous and normal tissue, the OSM expression data in the cancerous part was combined with other cancerous tissues. Similarly, the expression in the normal part was combined with other adjacent normal tissues for statistical analysis.

Statistical analysis: Assessments from multiple cores for each patient were averaged for epithelial cells and stage of malignancy (normal, DCIS, IDC, metastatic) that was present in the core. These four stages were statistically compared among ductal tissues. The OSM staining intensity was analyzed as a mixed model to accommodate repeated observations on each patient. These repeated observations were assumed to have non-negligible correlation and were modeled under standard repeated measures variancecovariance assumptions. Stage was treated as a fixed effect. Additionally, statistical models considering patient prognostic markers were evaluated. These models included the stage and the prognostic marker, with or without an interaction; the model with the lowest $\mathrm{AIC}_{\mathrm{c}}$ was selected to determine whether the prognostic factor was associated with 
OSM staining. These prognostic factors considered were age, tumor size, lymph node status, angiolymphatic invasion, tumor grade, tumor type, histologic grade, nuclear atypia, margin status, mitotic rate, Her2/neu expression, progesterone, and estrogen receptor. Initial assessments indicated that OSM staining intensity did not differ significantly between the two groups of patients (patients with and without lymph node metastasis) so patient group was not included as an analysis factor in the study. All models were assessed for adequacy by residual analysis, a concern here because of the bounds on OSM staining intensity (0-3) and our specific interest in changes in mean staining intensities. No predicted values exceeded the possible observational boundaries and residual patterns were acceptable despite the categorical nature of the data collection. All analyses were conducted using SAS version 9.1.3 (SAS 2004).

\section{$\underline{\text { Animal Histology }}$}

To verify lung metastasis, lungs from each experimental group (4T1.2-LacZ, n=2; 4T1.2-shOSM2, n=2; and 4T1.2-shOSM1, n=1) were placed in ultralight fixative (Ultralight Histology, Nampa, ID) paraffin embedded, and sectioned (Bi-Biomics, Nampa, ID). For each spine, four $1 \mu \mathrm{m}$ sections were collected $10 \mu \mathrm{m}$ apart in the lumbar region and H\&E stained. The sections were then stained overnight with 1:400 dilution of goat anti-mouse OSM primary antibody (Cat \# AF-495-NA; R\&D systems) and 1 hour with 1:1000 donkey-anti goat IgG-AP secondary antibody. The sections were then stained and imaged. 


\section{References}

1. C. Lange, E. Storkebaum, C. R. de Almodovar, M. Dewerchin, P. Carmeliet, Vascular endothelial growth factor: a neurovascular target in neurological diseases. Nat Rev Neurol 12, 439-454 (2016).

2. N. R. West, S. McCuaig, F. Franchini, F. Powrie, Emerging cytokine networks in colorectal cancer. Nat Rev Immunol 15, 615-629 (2015).

3. J. I. Murray, N. R. West, L. C. Murphy, P. H. Watson, Intratumoural inflammation and endocrine resistance in breast cancer. Endocr Relat Cancer 22, R51-67 (2015).

4. T. Deng, C. J. Lyon, S. Bergin, M. A. Caligiuri, W. A. Hsueh, Obesity, Inflammation, and Cancer. Annu Rev Pathol 11, $421-449$ (2016).

5. H. Zahid, E. R. Simpson, K. A. Brown, Inflammation, dysregulated metabolism and aromatase in obesity and breast cancer. Curr Opin Pharmacol 31, 90-96 (2016).

6. H. R. Harris, W. C. Willett, R. L. Vaidya, K. B. Michels, An Adolescent and Early Adulthood Dietary Pattern Associated with Inflammation and the Incidence of Breast Cancer. Cancer Res 77, 1179-1187 (2017).

7. Q. Chang et al., The IL-6/JAK/Stat3 feed-forward loop drives tumorigenesis and metastasis. Neoplasia 15, 848-862 (2013).

8. E. Angevin et al., A phase I/II, multiple-dose, dose-escalation study of siltuximab, an anti-interleukin-6 monoclonal antibody, in patients with advanced solid tumors. Clin Cancer Res 20, 2192-2204 (2014).

9. N. R. West et al., Oncostatin M drives intestinal inflammation and predicts response to tumor necrosis factor-neutralizing therapy in patients with inflammatory bowel disease. Nat Med 23, 579-589 (2017).

10. W. Hui, A. D. Rowan, C. D. Richards, T. E. Cawston, Oncostatin M in combination with tumor necrosis factor alpha induces cartilage damage and matrix metalloproteinase expression in vitro and in vivo. Arthritis Rheum 48, 3404-3418 (2003).

11. Q. Liu et al., Anti-OSM Antibody Inhibits Tubulointerstitial Lesion in a Murine Model of Lupus Nephritis. Mediators Inflamm 2017, 3038514 (2017).

12. A. R. Pradeep, T. M. S, G. Garima, A. Raju, Serum levels of oncostatin M (a gp 130 cytokine): an inflammatory biomarker in periodontal disease. Biomarkers 15, 277-282 (2010).

13. I. García-Tuñón et al., OSM, LIF, its receptors, and its relationship with the malignance in human breast carcinoma (in situ and in infiltrative). Cancer Invest 26, 222-229 (2008).

14. M. Esquivel-Velázquez et al., The role of cytokines in breast cancer development and progression. J Interferon Cytokine Res 35, 1-16 (2015).

15. C. L. Jorcyk, R. G. Holzer, R. E. Ryan, Oncostatin M induces cell detachment and enhances the metastatic capacity of T-47D human breast carcinoma cells. Cytokine 33, 323-336 (2006).

16. R. G. Holzer, R. E. Ryan, M. Tommack, E. Schlekeway, C. L. Jorcyk, Oncostatin $\mathrm{M}$ stimulates the detachment of a reservoir of invasive mammary carcinoma cells: role of cyclooxygenase-2. Clin Exp Metastasis 21, 167-176 (2004). 
17. C. Bolin et al., Oncostatin m promotes mammary tumor metastasis to bone and osteolytic bone degradation. Genes Cancer 3, 117-130 (2012).

18. M. M. Queen, R. E. Ryan, R. G. Holzer, C. R. Keller-Peck, C. L. Jorcyk, Breast cancer cells stimulate neutrophils to produce oncostatin M: Potential implications for tumor progression. Cancer Research 65, 8896-8904 (2005).

19. C. Tripathi et al., Macrophages are recruited to hypoxic tumor areas and acquire a Pro-Angiogenic M2-Polarized phenotype via hypoxic cancer cell derived cytokines Oncostatin M and Eotaxin. Oncotarget, (2014).

20. L. Lapeire et al., Cancer-associated adipose tissue promotes breast cancer progression by paracrine oncostatin M and Jak/STAT3 signaling. Cancer Res 74, 6806-6819 (2014).

21. R. E. Ryan et al., Oncostatin M binds to extracellular matrix in a bioactive conformation: implications for inflammation and metastasis. Cytokine 72, 71-85 (2015).

22. E. C. Walker et al., Murine Oncostatin M Acts via Leukemia Inhibitory Factor Receptor to Phosphorylate Signal Transducer and Activator of Transcription 3 (STAT3) but Not STAT1, an Effect That Protects Bone Mass. J Biol Chem 291, 21703-21716 (2016).

23. N. R. West, L. C. Murphy, P. H. Watson, Oncostatin M suppresses oestrogen receptor- $\alpha$ expression and is associated with poor outcome in human breast cancer. Endocr Relat Cancer 19, 181-195 (2012).

24. L. Humbert, M. Ghozlan, L. Canaff, J. Tian, J. J. Lebrun, The leukemia inhibitory factor (LIF) and p21 mediate the TGF $\beta$ tumor suppressive effects in human cutaneous melanoma. BMC Cancer 15, 200 (2015).

25. M. Murakami et al., IL-6-induced homodimerization of gp130 and associated activation of a tyrosine kinase. Science 260, 1808-1810 (1993).

26. D. A. Smith, A. Kiba, Y. Zong, O. N. Witte, Interleukin-6 and oncostatin-M synergize with the PI3K/AKT pathway to promote aggressive prostate malignancy in mouse and human tissues. Mol Cancer Res 11, 1159-1165 (2013).

27. D. J. Junk et al., Oncostatin M promotes cancer cell plasticity through cooperative STAT3-SMAD3 signaling. Oncogene, (2017).

28. P. C. Heinrich et al., Principles of interleukin (IL)-6-type cytokine signalling and its regulation. Biochem J 374, 1-20 (2003).

29. M. Kortylewski et al., Interleukin-6 and oncostatin M-induced growth inhibition of human A375 melanoma cells is STAT-dependent and involves upregulation of the cyclin-dependent kinase inhibitor p27/Kip1. Oncogene 18, 3742-3753 (1999).

30. C. Li, T. E. Ahlborn, F. B. Kraemer, J. Liu, Oncostatin M-induced growth inhibition and morphological changes of MDA-MB231 breast cancer cells are abolished by blocking the MEK/ERK signaling pathway. Breast Cancer Res Treat 66, 111-121 (2001).

31. N. R. West, J. I. Murray, P. H. Watson, Oncostatin-M promotes phenotypic changes associated with mesenchymal and stem cell-like differentiation in breast cancer. Oncogene 33, 1485-1494 (2014).

32. J. M. Smigiel, N. Parameswaran, M. W. Jackson, Potent EMT and CSC Phenotypes Are Induced By Oncostatin-M in Pancreatic Cancer. Mol Cancer Res 15, 478-488 (2017). 
33. S. L. Fossey, M. D. Bear, W. C. Kisseberth, M. Pennell, C. A. London, Oncostatin M promotes STAT3 activation, VEGF production, and invasion in osteosarcoma cell lines. BMC Cancer 11, 125 (2011).

34. R. Soldi et al., Oncostatin M activates phosphatidylinositol-3-kinase in Kaposi's sarcoma cells. Oncogene 9, 2253-2260 (1994).

35. S. Vollmer et al., Hypoxia-inducible factor 1alpha is up-regulated by oncostatin M and participates in oncostatin M signaling. Hepatology 50, 253-260 (2009).

36. M. Zhu et al., Oncostatin M activates STAT3 to promote endometrial cancer invasion and angiogenesis. Oncol Rep 34, 129-138 (2015).

37. P. R. Jay, M. Centrella, J. Lorenzo, A. G. Bruce, M. C. Horowitz, Oncostatin-M: a new bone active cytokine that activates osteoblasts and inhibits bone resorption. Endocrinology 137, 1151-1158 (1996).

38. P. Guihard et al., Oncostatin M, an Inflammatory Cytokine Produced by Macrophages, Supports Intramembranous Bone Healing in a Mouse Model of Tibia Injury. The American journal of pathology, (2015).

39. C. Bolin, C. Sutherland, K. Tawara, J. Moselhy, C. L. Jorcyk, Novel mouse mammary cell lines for in vivo bioluminescence imaging (BLI) of bone metastasis. Biol Proced Online 14, 6 (2012).

40. I. Martin-Padura et al., Spontaneous cell fusion of acute leukemia cells and macrophages observed in cells with leukemic potential. Neoplasia 14, 1057-1066 (2012).

41. B. L. Eckhardt et al., Genomic analysis of a spontaneous model of breast cancer metastasis to bone reveals a role for the extracellular matrix. Molecular Cancer Research 3, 1-13 (2005).

42. K. J. Livak, T. D. Schmittgen, Analysis of relative gene expression data using real-time quantitative PCR and the 2(-Delta Delta C(T)) Method. Methods 25, 402-408 (2001).

43. J. R. Garbow, J. P. Dugas, S.-K. Song, M. S. Conradi, A simple, robust hardware device for passive or active respiratory gating in MRI and MRS experiments. Concepts in Magnetic Resonance Part B: Magnetic Resonance Engineering 21B, 40-48 (2004).

44. R. H. Mak et al., Wasting in chronic kidney disease. J Cachexia Sarcopenia Muscle 2, 9-25 (2011).

45. Y. Imai et al., Injurious mechanical ventilation and end-organ epithelial cell apoptosis and organ dysfunction in an experimental model of acute respiratory distress syndrome. JAMA 289, 2104-2112 (2003).

46. J. Liu et al., Oncostatin M-specific receptor expression and function in regulating cell proliferation of normal and malignant mammary epithelial cells. Cytokine 10, 295-302 (1998).

47. G. Somlo et al., Multiple biomarker expression on circulating tumor cells in comparison to tumor tissues from primary and metastatic sites in patients with locally advanced/inflammatory, and stage IV breast cancer, using a novel detection technology. Breast Cancer Res Treat 128, 155-163 (2011).

48. H. Liu et al., The biological and clinical importance of epithelial-mesenchymal transition in circulating tumor cells. J Cancer Res Clin Oncol 141, 189-201 (2015). 
49. F. van Zijl, G. Krupitza, W. Mikulits, Initial steps of metastasis: cell invasion and endothelial transmigration. Mutat Res 728, 23-34 (2011).

50. Y. Husemann et al., Systemic spread is an early step in breast cancer. Cancer Cell 13, 58-68 (2008).

51. H. Hosseini et al., Early dissemination seeds metastasis in breast cancer. Nature, (2016).

52. L. D. Hazlett, R. S. Berk, Heightened resistance of athymic, nude (nu/nu) mice to experimental Pseudomonas aeruginosa ocular infection. Infection and immunity 22, 926-933 (1978).

53. J. Koch et al., Immune cells from SR/CR mice induce the regression of established tumors in BALB/c and C57BL/6 mice. PloS one 8, e59995 (2013).

54. A. M. Hicks et al., Transferable anticancer innate immunity in spontaneous regression/complete resistance mice. Proceedings of the National Academy of Sciences of the United States of America 103, 7753-7758 (2006).

55. S. Lauber et al., Novel function of Oncostatin $\mathrm{M}$ as a potent tumour-promoting agent in lung. International journal of cancer. Journal international du cancer 136, 831-843 (2015).

56. M. J. Tisdale, Mechanisms of cancer cachexia. Physiol Rev 89, 381-410 (2009).

57. K. B. Harvey, L. L. Moldawer, B. R. Bistrian, G. L. Blackburn, Biological measures for the formulation of a hospital prognostic index. Am J Clin Nutr 34, 2013-2022 (1981).

58. H. J. Patel, B. M. Patel, TNF- $\alpha$ and cancer cachexia: Molecular insights and clinical implications. Life Sci 170, 56-63 (2017).

59. A. Miller et al., Blockade of the IL-6 trans-signalling/STAT3 axis suppresses cachexia in Kras-induced lung adenocarcinoma. Oncogene 36, 3059-3066 (2017).

60. J. K. Onesti, D. C. Guttridge, Inflammation based regulation of cancer cachexia. Biomed Res Int 2014, 168407 (2014).

61. C. Li et al., Crosstalk between Platelets and the Immune System: Old Systems with New Discoveries. Adv Hematol 2012, 384685 (2012).

62. R. A. Ali, L. M. Wuescher, R. G. Worth, Platelets: essential components of the immune system. Curr Trends Immunol 16, 65-78 (2015).

63. S. Taucher et al., Impact of pretreatment thrombocytosis on survival in primary breast cancer. Thromb Haemost 89, 1098-1106 (2003).

64. C. K. Meikle et al., Cancer and Thrombosis: The Platelet Perspective. Front Cell Dev Biol 4, 147 (2016).

65. L. Yuan, X. Liu, Platelets are associated with xenograft tumor growth and the clinical malignancy of ovarian cancer through an angiogenesis-dependent mechanism. Mol Med Rep 11, 2449-2458 (2015).

66. B. Gligorijevic et al., N-WASP-mediated invadopodium formation is involved in intravasation and lung metastasis of mammary tumors. J Cell Sci 125, 724-734 (2012).

67. N. Bednarz-Knoll, C. Alix-Panabieres, K. Pantel, Clinical relevance and biology of circulating tumor cells. Breast Cancer Res 13, 228 (2011).

68. S. Zhao et al., Circulating tumor cells (CTCs) detected by triple-marker EpCAM, CK19, and hMAM RT-PCR and their relation to clinical outcome in metastatic breast cancer patients. Cell biochemistry and biophysics 65, 263-273 (2013). 
69. T. M. Gorges et al., Circulating tumour cells escape from EpCAM-based detection due to epithelial-to-mesenchymal transition. BMC Cancer 12, 178 (2012).

70. A. Gradilone et al., Circulating tumour cells lacking cytokeratin in breast cancer: the importance of being mesenchymal. Journal of cellular and molecular medicine 15, 1066-1070 (2011).

71. M. Zafrakas et al., Expression analysis of mammaglobin A (SCGB2A2) and lipophilin B (SCGB1D2) in more than 300 human tumors and matching normal tissues reveals their co-expression in gynecologic malignancies. BMC Cancer $\mathbf{6}$, 88 (2006).

72. A. M. Tester, N. Ruangpanit, R. L. Anderson, E. W. Thompson, MMP-9 secretion and MMP-2 activation distinguish invasive and metastatic sublines of a mouse mammary carcinoma system showing epithelial-mesenchymal transition traits. Clin Exp Metastasis 18, 553-560 (2000).

73. M. Y. Kim et al., Tumor self-seeding by circulating cancer cells. Cell 139, 13151326 (2009).

74. R. S. Muraoka et al., Increased malignancy of Neu-induced mammary tumors overexpressing active transforming growth factor beta1. Mol Cell Biol 23, 86918703 (2003). 
CHAPTER FOUR: HIGH EXPRESSION OF OSM AND IL-6 IS ASSOCIATED WITH DECREASED BREAST CANCER SURVIVAL: SYNERGISTIC INDUCTION OF IL-6 SECRETION BY OSM AND IL-1 $\beta$

\begin{abstract}
Authors
Ken Tawara, Hannah Scott, Jacqueline Emathinger, Cody Wolf, Celeste Bolin, Danielle Hedeen, Laura Bond, Cheryl Jorcyk
\end{abstract}

Boise State University, Boise, Idaho 83725

\begin{abstract}
Inflammation is driven by various immunomodulatory cytokines such as interleukin-6 (IL-6), oncostatin M (OSM), and interleukin-1 beta (IL-1 $\beta)$. Independently, each of these cytokines can promote in vitro metrics of cancer metastasis such as tumor cell detachment, epithelial-mesenchymal transition (EMT), and invasive potential. Furthermore, these cytokines promote breast cancer metastases to distant organs in in vivo mouse models. However, anti-IL-6 and anti-IL-1 $\beta$ therapeutics have not yielded significant results against solid tumors in clinical trials. Only anti-OSM therapies remain untested against human cancer progression. Here we show that these three cytokines are
\end{abstract}


interrelated in function, which may explain why single anti-cytokine therapies have been ineffective. Using Oncomine ${ }^{\mathrm{TM}}$ data, we have determined that there is a correlation between high co-expression levels of OSM, IL-6, and IL-1 $\beta$ and reduced breast cancer patient survival. Furthermore, we confirm that OSM induces the expression of IL-6 in breast cancer in a manner that is dependent on both an estrogen receptor-negative (ER-) status, as well as STAT3 signaling. In addition, OSM and IL-1 $\beta$ synergistically induce IL-6 secretion using independent signaling pathways in MDA-MB-231 and MCF7 human breast cancer cells. Specifically, OSM signals through STAT3 and ERK, while IL-1 $\beta$ signals through p65 and ERK. This study demonstrates that OSM, IL-6, and IL-1 $\beta$ expression levels in breast cancer patient data are correlated, suggesting a complex interplay between these cytokines in tumor progression. Importantly, we provide a rationale for a breast cancer treatment regime that simultaneously targets these three proteins, as these cytokines appear to independently possess many overlapping functions that increase metastasis and worsen patient survival.

\section{Introduction}

Breast cancer-related morbidity and mortality remains one of the top concerns for women worldwide with 252,710 new cases of breast cancer predicted for 2017 (1). Despite new treatments and extensive preventative screening initiatives, the breast cancer incidence rate has remained flat, and there has been little improvement in the survival rate for stage 4 metastatic breast cancer (2). Major breast cancer risk factors include metabolic abnormalities and central obesity where there is extensive adipose tissue accumulation in the midsection (3). Obesity incidence rates have been increasing in the developed world and there are strong associations between obesity, cancer, and 
inflammation, suggesting increased rates of breast cancer with increasing obesity (4-7). Inflammatory cytokines such as interleukin-6 (IL-6), tumor necrosis factor-alpha, (TNF $\alpha$ ), and interleukin-1 beta (IL-1 $\beta$ ) have been linked to tumor invasion and metastasis (8). IL-6, in particular, has been associated with increased breast tumor cell proliferation, metastatic capacity, and decreased patient survival rates (9). Although recent research has shown the importance of IL-6 in cancer disease progression, anti-IL-6 therapies have not produced clinically beneficial results for the treatment of solid tumors $(10,11)$. This suggests a redundancy where other pro-inflammatory mediators further contribute.

The IL-6 cytokine is a part of the gp130 family of cytokines which include IL-6, oncostatin M (OSM), leukemia inhibitory factor (LIF), IL-31, IL-11, IL-27, cardiotrophin-1, ciliary neurotrophic factor, and cardiotrophin like cytokine (12). These cytokines have a shared gp130 receptor subunit and have a wide range of functions in cancer and inflammation (12-14). OSM, in particular, has been shown to induce tumor cell detachment, invasion, EMT, induction of cancer stem cells, immune evasion, and osteolytic bone metastases, (15-21). OSM is thought to exert much of its function through binding to the OSM receptor (OSMR), a gp130/ OSMR $\beta$ complex, to induce downstream signaling pathways such as signal transducer and activator of transcription 3 (STAT3), mitogen-activated protein kinase (MAPK), and AKT (22-24).

OSM has been known to synergize with interleukin-1 (IL-1 $\alpha$ ) and IL-1 $\beta$ in the context of cartilage breakdown in the joint, showing an amplified induction of matrix metalloproteinases (MMPs), IL-8, as well as IL-6 (25-27). Additionally, OSM and IL-1 $\beta$ have been shown to synergistically induce vascular endothelial growth factor (VEGF) in 
astroglioma cells (28). Both IL- $1 \alpha$ and IL-1 $\beta$ activate the same IL-1 receptor, (a dimer of IL-1R1 and IL-1RAcP), while IL- $1 \alpha$ is a membrane bound protein and IL-1 $\beta$ is a soluble protein (29). IL-1 $\beta$ increases tumor invasiveness, immunosuppression, and progression of metastatic disease $(29,30)$. IL-1 $\beta$ is able to promote these effects through the activation of p65 and ERK and induce the production of nitric oxide and proteinases (31-33). Similar to our studies with OSM and breast cancer metastasis to bone (18), IL-1 $\beta$ also stimulates the development of bone metastases (34). Unfortunately, anti-IL-1 $\beta$ therapies such as anakinra (Kineret $\left.{ }^{\mathrm{TM}}\right)$ have not demonstrated significant effects against solid tumors, although additional clinical trials are in progress to investigate its effectiveness further (35-37). Together, these studies suggest that there are many overlapping functions between IL-6, OSM, and IL-1 $\beta$ that expose possible weaknesses in the use of singular anti-cytokine therapies.

Gene expression studies on breast cancer subtypes and immunological factors such as TLR9, and signaling factors such as STAT3, MAPK, and AKT suggest that there may be sub-type specific differences in the level of expression and activation of downstream pathways $(38,39)$. This may inform that there are possible breast cancer subtype specific differences in cytokine signaling, which would further complicate possible therapeutic interventions using anti-cytokine biologics. Subtyping breast cancer relies on the positive or negative detection of estrogen receptor (ER) and progesterone receptor (PR), as well as the expression level of human epidermal growth factor receptor 2 (HER2) and Ki67 (40). Specifically, breast cancer cells that are classified as luminal A $\left(\mathrm{ER}+/ \mathrm{PR}+/ \mathrm{Her} 2^{\text {low }}\right)$ are the less aggressive and have good prognosis overall, while triple negative (ER-/PR-/Her2 ${ }^{\text {low }}$ ) are much more aggressive (41). OSM have been shown to 
suppress ER expression in luminal A cells, and drive them into a more metastatic phenotype $(42,43)$. In particular high expression of OSMR has been correlated with low survival rates, which suggest that OSM signaling in general may promote breast cancer progression (42). Other studies suggest that OSM may be higher in triple negative compared to luminal A or B breast cancer phenotypes (44). Collectively, these studies suggest that OSM expression and signaling may be correlated with breast cancer subtypes, and that subtype specific breast cancer response to cytokines may drive breast cancer disease progression.

In this study, we investigate the effect of OSM, IL-6, and IL-1 $\beta$ on breast cancer patient survival as well as how these cytokines are interrelated in terms of cell signaling. Using Oncomine ${ }^{\mathrm{TM}}$ data, we assessed the correlation between OSM and IL-6 with regards to patient survival. Specifically, we found that high expression of OSM or IL-6 was correlated to significantly decreased breast cancer patient survival. Furthermore, we found distinct signaling differences between breast cancer subtypes in terms of OSM induction of IL-6 in vitro with ER- MDA-MB-231 (ER-/PR-/Her2 $\left.{ }^{\text {low }}\right)$, ER+ T47D $\left(\mathrm{ER}+/ \mathrm{PR}+/ \mathrm{Her} 2^{\text {low }}\right)$, and $\mathrm{ER}+\mathrm{MCF} 7\left(\mathrm{ER}+/ \mathrm{PR}+/ \mathrm{Her} 2^{\text {low }}\right)$ cells. Interestingly, OSM induction of IL-6 only occurred in the ER- MDA-MB-231 cells through the STAT3 signaling pathway and not in the two ER+ cells lines tested. We also reveal that cotreatment of breast cancer cells with both OSM and IL-1 $\beta$ led to a synergistic increase in IL-6 secretion. These results highlight that OSM and IL-1 $\beta$ synergistically increase IL-6 in breast cancer, promote a more metastatic phenotype, and reduce breast cancer patient survival. 


\section{Materials and Methods}

$\underline{\text { Oncomine analysis }}$

The Curtis human breast cancer mRNA microarray dataset (45) and the Finak breast cancer stromal gene expression mRNA dataset (46) were obtained from Oncomine $^{\mathrm{TM}}$ (Compendia Bioscience, Ann Arbor, MI). For survival analysis, the Curtis dataset was filtered for "Invasive Ductal Carcinoma" and valid "Alive" or "Dead of Disease" status. From the filtered dataset, upper $\left(>75^{\text {th }}\right.$ percentile $)$, and lower $\left(<25^{\text {th }}\right.$ percentile) quartiles of gene expression for OSM, IL-6, and IL-1 $\beta$ were selected for comparison. For multi-gene co-expression analysis, we calculated for patients high in both OSM and IL-6, or OSM and IL-1 $\beta$. Survival statistical analysis was performed using a log-rank test in Graphpad Prism 5 software.

To analyze gene correlations, the Curtis dataset was subjected to a correlation analysis using Graphpad Prism 5 software and the RealStatistics package on Microsoft Excel. To assess gene expression levels of OSM and IL-1 $\beta$ in stromal tissue, patients in the Finak dataset were separated into normal and breast cancer categories, as well as into ER+ and ER- breast cancer categories. Additionally, the Curtis dataset was also separated into ER+ and ER- breast cancer categories to assess gene expression levels in the tumor cells. Statistical analysis was performed using the 2-tailed student's T-test in Graphpad Prism 5 software.

Enzyme linked immunosorbent assay

Serum was collected from breast cancer patients of various stages at St. Luke's Mountain States Tumor Institute (MSTI) in accordance with the institutional review board or purchased from Proteogenix (Schiltigheim, France) and Bioreclamations 
(Baltimore, Maryland). The serum was diluted 1:3 in PBS and used in the DuoSet ELISA for IL-6 (DY206, R\&D system) or for OSM (DY295, R\&D systems) on Immulon HBX4 ELISA plates (3855, ThermoFisher) in accordance with the manufacturer's instructions. Data was analyzed using Graphpad prism 5.0 to determine correlations between OSMIL-6 serum concentrations.

Cells were incubated with OSM or IL- $1 \beta$ treatments $(10 \mathrm{ng} / \mathrm{mL})$ ranging from 48 72 hours and the resultant cytokine levels were assessed. To measure human IL-6 and OSM in conditioned media, the same R\&D ELISA kits were utilized according to the manufacturer's instructions. Conditioned media from MDA-MB-231 cells were diluted 1:10 to $1: 20$ in order to accurately detect the amount of IL-6 within the range of the standard. Conditioned media was collected from 24-well plates containing $1 \times 10^{5}$ cells at the time of cell plating.

\section{$\underline{\text { Cell lines }}$}

MDA-MB-231, T47D, MCF7, and MDA-MB-468 human breast cancer cells, PC3 and DU145 human prostate cancer cells, and HeLa human cervical cancer cells were obtained from the American Type Culture Collection (Rockville, MD). All human cell lines were maintained in RPMI 1640 media supplemented with $10 \%$ fetal bovine serum and to 100 units/mL of streptomycin and penicillin (Hyclone, Logan UT). 4T1.2 mouse mammary carcinoma cells were maintained in MEM-alpha media supplemented with $10 \%$ fetal bovine serum and to 100 units/mL of streptomycin and penicillin. All cells, and experimental incubations were maintained at $37^{\circ} \mathrm{C}, 5 \%$ carbon dioxide, and $100 \%$ humidity in a water-jacketed cell culture incubator. 


\section{$\underline{\text { Stable and transient transfections }}$}

To generate stably transduced MDA-MB-231 Luc2 D3H2LN cells (Caliper Life Sciences) with inducible expression of OSM, the OSM cDNA (862 bp) (A generous gift from Dr. Atsushi Miyajima, The University of Tokyo) was cloned into the pLenti 6.3/TO/V5-DEST vector (A11144 ThermoFisher). The vector+hOSM was then cotransduced with pLenti3.3/TR (A11144, ThermoFisher) into MDA-MB-231 Luc2 D3H2LN cells using the ViraPower ${ }^{\mathrm{TM}}$ II Lentiviral Gateway ${ }^{\circledR}$ Expression System (K367-20, Life technologies) using the manufacturer's instructions. Stably transduced cells were injected into mice and resultant tumors and animal sera tested for TET induction by western blot and ELISA. The stable TET inducible OSM expressing MDAMB-231 Luc2 D3H2LN clone has been designated as MDA ${ }^{\mathrm{TO} / O S M}$.

To generate stable expression of estrogen receptor alpha (ER $\alpha)$ in ER- MDA-MB231 cells, an ER $\alpha$ expressing plasmid (Cat\# 28230, AddGene) was stably transfected into MDA-MB-231 cells using Lipofectamine LTX (Cat\# 15338100, Life Technologies). For control cells, an empty pEGFP-C1 (Cat\#6084-1, Clontech) vector was stably transfected into these cells. Cells were transfected at $80 \%$ confluency in 96-well plates containing RPMI 1640+10\% FBS with $6 \mu \mathrm{g}$ DNA per well and a lipofectamine:DNA ratio of 1.35:1. Transfected cells were selected for using G418 at a concentration of $500 \mu \mathrm{g} / \mathrm{mL}$. Surviving colonies were expanded under antibiotic pressure and their expression of ER $\alpha$ was verified by western blot analysis.

To transiently suppress STAT3, a siRNA pool targeting STAT3 was purchased from Dharmacon (Cat \#L-003544-00-0005). 100,000 cells per well were seeded in a 24well plate, and the siRNA was transfected in accordance to the Fast-Forward protocol as 
per the technical manual included with the Hyperfect siRNA transfection reagent (Cat\# 301705, Qiagen). STAT3 siRNA was used at $25 \mathrm{nM}$ and the cells were transfected for 72 hours before being treated with OSM or IL-1 $\beta$. Knockdown of STAT3 was assessed by western blot.

$\underline{\text { Animal tumor xenograft model }}$

Six-week old female athymic nude mice were purchased from the NCI Animal Production Facility (Fredrick, MD). The MDA ${ }^{\text {TO/OSM }}$ cells were grown to $90 \%$ confluency and the cells were concentrated to $4.0 \times 10^{7}$ cells $/ \mathrm{mL}$ in PBS containing $10 \%$ RPMI 1640 , and $50 \mathrm{uL}$ of the cell suspension was injected into the $4^{\text {th }}$ mammary fat pad. When the tumors became palpable, the animals were given drinking water containing tetracycline in $2 \%$ sucrose water for one week with doses ranging from $0 \mathrm{mg} / \mathrm{mL}, 0.1$ $\mathrm{mg} / \mathrm{mL}$ and $1 \mathrm{mg} / \mathrm{mL}$. Animals were sacrificed and their serum and tumors collected for analysis.

$\underline{\text { Immunoblot assay }}$

Cells were plated on 24-well plates at 70-80\% confluency and allowed to adhere overnight at $37^{\circ} \mathrm{C}$. Cells were treated with cytokines, OSM (CAT\#300-10T) and/or IL1Beta (CAT\# 200-01B) (Peprotech), and with inhibitors, the ERK inhibitor PD98059 (CAT\# 9900, Cell Signaling) or the p65 inhibitor caffeic acid phenyl ester (CAPE) (CAT\# 2743, Tocris). Cells were treated for 30 minutes or 72 hours. Conditioned media was collected from the cells treated for a 72 hours, and cell lysates were collected from both time points using 1x RIPA buffer containing a protease inhibitor cocktail (Sigma Aldrich, CAT\# P8340). Lysates were run on an SDS-PAGE gel and transferred to nitrocellulose immunoblot membranes via semi-dry transfer. Blots were rinsed in $\mathrm{ddH} 2 \mathrm{O}$ 
and allowed to completely dry before being blocked with PBS-T (PBS, pH 7.4; Tween20,0.05\%; 5\% non-fat dry milk) for 1 hour. After 3 x 5 min PBS-T washes, primary antibodies (1:1000) suspended in PBS-T complemented with 1\% BSA were then applied to the membrane and incubated overnight at $4^{\circ} \mathrm{C}$. After another 3 x 5 min PBS-T washes, horse radish peroxidase conjugated secondary antibodies suspended in PBS-T were then applied to the membrane. Then with a final 5 x 5 min PBS-T wash, the membrane was developed with enhanced chemiluminescence and imaged using Syngene G:BOX imager. All antibodies used for the immunoblots were acquired from Cell Signaling Technologies. STAT3 (CAT\#9132), phospho-STAT3 (Y705) (CAT\# 9145), Beta-Actin

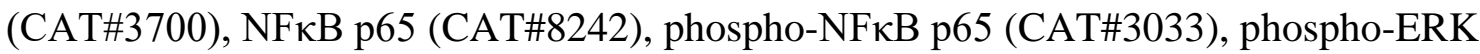
(CAT\# 4370), Anti-rabbit IgG-HRP (CAT\# 7076).

\section{Immunoprecipitation}

MCF7 and T47D cells were incubated with $10 \mathrm{ng} / \mathrm{mL}$ of OSM and/or IL-1 $\beta$ for 48 hours at a density of 100,000 cells per well in a 24-well plate. Cells were lysed with Cell Signaling PathScan ${ }^{\circledR}$ Lysis buffer (Cat\# 7018) using the manufacturer's instructions. The lysates were then used on a Dynabeads ${ }^{\circledR}$ Protein A Immunoprecipitation Kit (Cat\# 10006D, Life Technologies) using ER $\alpha$ IP antibody at 1:50 dilution from Cell Signaling (Cat\# D8H8) in accordance with the kit instructions with the following modification. In order to reduce co-elution of the antibody, the antibody was cross-linked using $20 \mathrm{mM}$ dimethyl pimelimidate dihydrochloride (Cat\# 21666, Pierce) in $0.2 \mathrm{M}$ triethanolamine at a pH of 8.2. The antibody-bead complex was cross-linked for 30 minutes, and the reaction was stopped by resuspending the beads for 
15 minutes in $50 \mathrm{mM} \mathrm{pH7.5} \mathrm{Tris.} \mathrm{The} \mathrm{beads} \mathrm{were} \mathrm{then} \mathrm{used} \mathrm{in} \mathrm{the} \mathrm{rest} \mathrm{of} \mathrm{the}$ immunoprecipitation protocol following a 3x PBS-0.05\% Tween-20 wash.

$\underline{\text { Statistical analysis }}$

All the statistical analyses were performed using GraphPad Prism version 5 software or with the RealStatistics ${ }^{\mathrm{TM}}$ package for Microsoft Excel. To compare multiple groups, one- or two-way ANOVA was performed using Tukey's post-test where appropriate. Comparisons between two groups were assessed by unpaired two-tailed student's t-test. Correlations were assessed using the Spearman nonparametric correlation analysis. Survival data was assessed using the Log-rank test. Statistical significance was assigned to experimental $p$ values that were less than 0.05 . Error bars represent the standard error of the mean unless otherwise specified, and all experiments were performed at least three times.

\section{Results}

OSM and IL-6 expression are correlated with each other and associated with decreased $\underline{\text { invasive breast cancer survival }}$

Both OSM and IL-6 have previously been shown to increase breast cancer metastatic potential in vitro as well as promote metastasis in vivo $(9,18,22,42,47-51)$, which suggests that these cytokines may negatively affect patient survival. In particular, serum IL-6 levels have been correlated with reduced breast cancer patient survival (52). To assess the relevance of tumor tissue expression of OSM and IL-6 in the context of invasive breast cancer patient survival, we used the Curtis Breast dataset obtained from Oncomine TM (45). The upper quartile was delineated as the top $25 \%$ of patient expression levels (high expression), while the lower quartile represents the bottom $25 \%$ 
expression (low expression). High tumor tissue expression of OSM (p<0.001, Fig. 1A) and IL-6 (p<0.001, Fig. 1B) both correlated with a significant decrease in survival. As this data demonstrates that OSM and IL-6 independently correlates with decreased survival in invasive breast cancer patients, we next assessed whether co-expression of high levels of OSM and IL-6 is also correlated with decreased survival. Indeed, high coexpression of both OSM and IL-6 were significantly correlated with decreased survival compared to low co-expression of both OSM and IL-6 (p=0.0091, Fig. 1C). Further assessment revealed that expression of OSM correlates with IL-6 with a Spearman coefficient of 0.576 (p<0.0001, Fig. 1D). Collectively, this suggests that elevated breast cancer tissue levels of OSM and IL-6 leads to decreased survival and that their expression is correlated with each other. This kind of co-expression is indicative of potential coregulation and suggests that these cytokines may induce the expression of each other. $\underline{\text { High OSM serum levels in breast cancer patients are correlated high IL-6 levels }}$

Previous studies suggest that aberrant expression of growth factors and cytokines in the tumor microenvironment (TME) can result in these proteins leaking into the circulation and become detectable in patient serum $(53,54)$. To assess whether serum concentrations of OSM and IL-6 also show a correlation, as seen with the Curtis Breast dataset in Figure 1D, we assessed serum samples collected from breast cancer patients and healthy individuals by ELISA. Breast cancer patient serum had significantly higher levels of OSM and IL-6 compared to serum from individuals without malignancies (Fig. 2A, B). Next, we analyzed the serum sample data to test whether OSM serum concentrations are correlated to IL-6 concentrations. In samples with no OSM, there was very little IL-6 in the serum, while any serum sample with $>0 \mathrm{pg} / \mathrm{mL}$ concentration of 
OSM had a significantly higher level of IL-6 (Fig. 2C). Furthermore, a serum OSM and IL-6 concentration correlation analysis reveal that there is a Spearman coefficient of 0.3774 (p<0.0001, Fig. 2D). These results suggest that patients with breast cancer have elevated serum OSM and IL-6 levels compared to normal patients and that the serum concentrations of these cytokines correlate with each other similarly to the tumor expression levels of OSM and IL-6 seen with the Curtis dataset.

OSM promotes IL-6 expression in a mouse model of human breast cancer

In order to recapitulate potential OSM-induced IL-6 expression in breast cancer, we utilized an MDA-MB-231 orthotopic mouse model of human breast cancer. MDAMB-231-Luc2 cells were stably transfected with a TET-inducible OSM expression vector, and these $\mathrm{MDA}^{\mathrm{TO} / \mathrm{OSM}}$ cells were orthotopically injected into athymic nude mice into the $4^{\text {th }}$ mammary fat pad. Once the tumors were palpable $\sim 3-5 \mathrm{~mm}$, the animals were given drinking water with $2 \%$ sucrose containing $0.1 \mathrm{mg} / \mathrm{mL}$ tetracycline (+TET) or $2 \%$ sucrose water alone (-TET). MDA ${ }^{\mathrm{TO} / O S M}$ tumor bearing animals +TET had a 32-fold higher expression of OSM in their tumors compared to -TET tumors (Fig 3A) and 10.8fold higher IL-6 expression level (Fig 3B) as measured by western blot analysis. After collecting blood, OSM and IL-6 serum levels were assessed by ELISA. TET- treated mice had higher levels of OSM (9.8-fold, Fig 3C Left) and IL-6 (96-fold, Fig 3C Right) in their serum, and the concentrations correlated with each other with an $r^{2}$ coefficient of 0.9058 ( $\mathbf{p = 0 . 0 0 3 4}$, sFig 1). Collectively, TET induction in these mice led to increased levels of OSM and IL-6 in both the tumors and serum, which concurs with the breast cancer patient serum data and the Curtis breast cancer tumor expression data from 
Oncomine. This also suggests that elevated cytokine serum levels may be indicative of high cytokine expression levels in the tumor microenvironment (TME).

OSM induces human IL-6 secretion in the absence of ER from various cancer cells in $\underline{\text { vitro }}$

Our results indicate that there is strong inter-correlation between OSM and IL-6 expression and secretion levels in breast cancer. To assess the nature of cytokine production in breast cancer cells, various cell lines including two human ER+ cell lines, T47D and MCF7, and three ER- cell lines MDA-MB-468, MDA-MB-231, and 4T1.2 mouse mammary cancer cells were treated with OSM, and their IL-6 secretion was assessed. In addition, PC3 and Du145 human prostate cancer cells as well as HeLa human cervical carcinoma cells were tested for OSM-induced IL-6 secretion. Recombinant hOSM (25 ng/mL) was used to treat the human cell lines, while recombinant mouse OSM (rmOSM; $25 \mathrm{ng} / \mathrm{mL}$ ) was used for the 4T1.2. The cells were treated with OSM for 48 hours, and IL-6 levels in the conditioned media (CM) were assessed by ELISA. Interestingly, OSM did not induce IL-6 secretion in the ER+ MCF7 or T47D cells but did in the ER- cells (Fig. 4A). OSM promoted IL-6 secretion approximately 5-fold in MDA-MB-468 cells, 4-fold in MDA-MB-231 cells, and 4-fold in 4T1.2 mouse mammary carcinoma cells (Fig 4A). Androgen receptor-negative (AR-) PC3 cells express high levels of IL-6 with or without OSM, while OSM induced IL-6 approximately 6.5-fold in the AR- Du145 cells (Fig 4B). Also in the ER- HeLa cells, IL6 levels were undetectable in the absence of OSM treatment, but the cells produced 1245 $\mathrm{pg} / \mathrm{mL}$ of IL-6 with OSM treatment (Fig 4B). On the other hand, IL-6 had no effect on OSM secretion in MDA, T47D, or MCF7 cells (sFig 2), which suggest that there is no 
reciprocal induction of cytokine secretion. These results show that OSM increased IL-6 expression in cells that are more aggressive and may correlate to estrogen receptor status.

To assess whether the presence of ER is essential for OSM induction of IL-6, ERMDA-MB-231 cells were stably transfected with an ER expression vector (pEGFP-C1). Two independent colonies, $\mathrm{MDA}^{\mathrm{ER}+/ \mathrm{C} 7}$, and $\mathrm{MDA}^{\mathrm{ER}+/ \mathrm{H} 6}$, were shown to express $\mathrm{ER}$ by western blot analysis (Fig 4C). Parental MDA-MB-231 cells, $\mathrm{MDA}^{\mathrm{ER}+/ \mathrm{C} 7}$, and $\mathrm{MDA}^{\mathrm{ER}+\mathrm{H} 6}$ were treated with rhOSM $(25 \mathrm{ng} / \mathrm{mL})$ for 48 hours to assess their IL-6 secretion. The CM was then collected and their IL-6 concentrations were analyzed by ELISA. $\mathrm{MDA}^{\mathrm{ER}+/ \mathrm{C} 7}$ cells exhibited a 7.8-fold decrease and $\mathrm{MDA}^{\mathrm{ER}+/ \mathrm{H} 6}$ cells demonstrated a 12.1-fold decrease in the levels of IL-6 produced in response to OSM, as compared to the parental MDA-MB-231 cells (Fig 4D). This result indicates that the ER+ MDA-MB231 cells have limited OSM-induced IL-6 expression and suggests that ER may play a negative regulatory role in OSM signaling that leads to IL-6 expression.

STAT3 is phosphorylated by OSM signaling in both ER- and ER+ cells

Previous studies show that IL-6 induction is dependent on the activation of NFאB and STAT3 signaling pathways (55). MDA-MB-231, T47D, and MCF7 were treated with $25 \mathrm{ng} / \mathrm{mL}$ OSM for 5 and 30 minutes to assess which signaling pathways are activated in response to OSM in these cells. STAT3, AKT, ERK, and JNK pathways were activated by OSM in all three cell lines, however only MDA-MB-231 cells produced IL-6 in response to OSM (Fig 5A). This suggests that despite the nearly uniform activation of OSM-induced pathways in both ER+ and ER- cells, there must be something else occurring that is suppressing IL-6 production in the ER+ cells. Further investigation using siRNA (20 nM) against STAT3 completely abrogated OSM-induced IL-6 secretion 
in MDA-MB-231 cells as detected by immunoblot (Fig 5B) and ELISA (Fig 5C), thereby implicating STAT3 as the primary signaling pathway responsible for OSM induction of IL-6 secretion. This also demonstrates that STAT3 is not able to induce IL-6 expression in ER+ T47D or MCF7 cells despite the apparent phosphorylation of STAT3 by OSM. $\underline{\text { Invasive breast cancer patient stroma expresses high levels of OSM and IL-1 } \beta}$

IL-1 $\beta$ has been known to synergize with OSM in the context of inflammatory conditions such as arthritis and progressive joint deterioration $(25,56)$ and has been shown to induce the expression of IL-6 in vitro (57). As inflammation appears to have negative effects on breast cancer patient survival, we assessed patient data related to these cytokines. In particular, stromal cytokines in the breast cancer microenvironment appear to play a major role in the progression of metastatic disease (58). We assessed OSM and IL-1 $\beta$ stromal expression in both normal and invasive breast cancer patient data using the Finak Breast Stromal dataset (46) obtained from Oncomine ${ }^{\mathrm{TM}}$. Stromal OSM expression was 5.9-fold higher in invasive breast cancer compared to normal samples (Fig 6A), and stromal IL-1 $\beta$ was 5.4-fold higher in invasive breast cancer compared to normal samples (Fig 6B). There was also a significant increase in stromal OSM (sFig 3A) and IL-1 $\beta$ expression (sFig 3B) in ER- samples compared to ER+ samples. Using the Curtis dataset, a significant increase in tumor OSM expression (sFig 3C) as well as tumor IL-1 $\beta$ (sFig 3D) expression was seen in ER- samples compared to ER+ samples. These data suggest that in breast cancer patients both paracrine and autocrine production of OSM and IL-1 $\beta$ may work in an ER-dependent manner to affect patient survival. 
$\underline{\text { OSM and IL-1 } \beta \text { promote increased lymph node metastases and decreased survival: a }}$ three-way correlation for OSM-IL-1 $\beta$-IL-6 expression

The correlation between tumor OSM and IL- $1 \beta$ expression and metastatic capacity was assessed in the Curtis patient dataset. A 2.1-fold increase in lymph node metastasis seen with high OSM versus low OSM expression for invasive breast cancer (Fig 6C Left). Similarly, lymph node metastasis was higher in patients with high IL-1 $\beta$ expression (Fig 6C Center). Furthermore, when both OSM and IL-1 $\beta$ co-expression was high, there was also a significantly higher number of lymph node metastases compared to the low OSM and IL-1 $\beta$ co-expression group (Fig 6C Right). High expression of OSM and IL-1 $\beta$ also led to decreased patient survival (Fig 6D). In addition, OSM expression levels correlated with IL-1 $\beta$ expression level (sFig 4A) and IL-1 $\beta$ expression levels correlated with IL-6 expression level (sFig 4B). To determine whether all three cytokines were correlated with each other, OSM, IL-6, and IL-1 $\beta$ expression levels were assessed using a least squares multiple correlation analysis (Fig 6E). The three-way correlation coefficient of OSM, IL-6, and IL-1 $\beta$ was 0.6001 , with a p-value of $2.2 \times 10^{-23}$, indicating significant correlation between the three cytokines in this analysis. Collectively, these results suggest that OSM, IL-6, and IL-1 $\beta$ are interrelated in breast cancer patient metastasis and survival. These results demonstrate that not only does each one of these cytokines increase metastasis and decrease survival in breast cancer patients but also induce the expression of other cytokines as well. This may provide at least in part, an explanation as to why clinical trials using single anti-cytokine therapies thus far have failed. 
OSM works synergistically with IL-1 $\beta$ to promote IL-6 secretion

We have shown that high OSM, IL-6, and IL-1 $\beta$ levels all correlate with decreased breast cancer patient survival, and that high expression levels of these three cytokines correlate with each other in patient samples. Therefore, it is possible that OSM and IL-1 $\beta$ may work synergistically to promote IL-6 expression and secretion. To better assess the relationship between the two cytokines, we decreased the amount of OSM and IL- $1 \beta$ used in the experiments from $25 \mathrm{ng} / \mathrm{mL}$ to $10 \mathrm{ng} / \mathrm{mL}$, thus reducing the probability of saturating the cell's capacity to produce IL-6. Treating ER- MDA-MB-231 cells with a combination of OSM and IL- $1 \beta$ for 72 hours resulted in a 44.8 -fold increase in IL-6 secretion by ELISA, while OSM alone lead to a 6.4-fold increase and IL-1 $\beta$ alone lead to a 17.3-fold increase compared to not-treated cells (Fig 7A). This suggests that OSM and IL-1 $\beta$ induce IL-6 production in a synergistic manner in ER- cells. No induction of IL-6 secretion by OSM was seen in either ER+ T47D or MCF7 cells. After adjusting the scale for IL-6 secretion levels, an increase in IL-6 secretion by IL-1 $\beta$ was seen in ER+ MCF7 cells, as compared to untreated controls (Fig 7B). MCF7 cells also exhibited a synergistic 24.8-fold increase in IL-6 secretion by treatment with both OSM and IL-1 $\beta$ compared to IL-1 $\beta$ alone (Fig 7B). Although the level of IL-6 production is much lower in the ER+ MCF7 cells compared to the ER-MDA-MB-231 cells, there is a clear indication of a synergistic relationship between OSM and IL-1 $\beta$ in this cell line as well. T47D cells, on the other hand, were unable to produce any IL- 6 in response to OSM or IL-1 $\beta$, even though their activity was confirmed by an increased EMT-like morphology and invadopodia formation (sFig 5). This indicates that even if a breast cancer cell line like 
T47D is unable to produce IL-6 in response to OSM or IL-1 $\beta$, it can still promote invasive characteristics independently of IL-6 (20).

OSM and IL-1 $\beta$ activate separate signaling pathways to promote IL-6 production

The synergistic upregulation of IL-6 secretion in response to OSM and IL-1 $\beta$ suggests that these cytokines may be using separate pathways to promote IL-6 in breast cancer cells. Our data here and previously published work demonstrate that IL-1 $\beta$ utilizes the NFkB pathway and OSM uses the STAT3 pathway to induce IL-6 production (55). Interestingly, a past study indicated that there is crosstalk between STAT3 and NFkB to promote downstream effects (59). To assess gross signaling pathway differences between OSM and IL-1 $\beta$ on MDA-MB-231, T47D, and MCF7 cells, the cells were treated with both cytokines (10 ng/mL) for 20 minutes (Fig 7C) or 72 hours (Fig 7D). In all breast cancer cell lines tested, OSM specifically induced STAT3 phosphorylation, while IL-1 $\beta$ induced p65 phosphorylation. This suggests that OSM may primarily induce IL-6 expression through STAT3 activation, while IL- $1 \beta$ induces IL-6 expression through the activation of the NFkB pathway. Additionally, ERK phosphorylation was unaffected in ER- MDA-MB-231 cells, but both OSM and IL-1 $\beta$ increased phospho-ERK in both T47D and to a lesser extent, in MCF7 cells. This data hints that there may be some ERK signaling related aspects to IL-6 secretion as both OSM and IL-1 $\beta$ affected ERK phosphorylation. To investigate the involvement of the ERK pathway, a small molecule inhibitor against ERK (PD98059) was used (sFig 6A). While MDA-MB-231 cells were unaffected by ERK inhibition (sFig 6B), IL-1 $\beta$ and OSM+IL-1 $\beta$ induced IL-6 secretion in MCF7 cells was significantly reduced (sFig 6C). Therefore, IL-1 $\beta$-mediated induction 
of IL-6 in MCF7 cells may utilize the ERK signaling pathway in addition to the NFאB pathway, while only the NFאB pathway is used in MDA-MB-231 cells,

Another signaling difference between the cell lines was that OSM-induced STAT3 phosphorylation was much weaker in MCF7 cells compared to T47D or MDAMB-231 cells (Fig 7C and D). This could suggest that IL-6 induction by OSM and IL-1 $\beta$ might not be dependent on STAT3 activation in MCF7 cells unlike with MDA-MB-231 cells. To confirm that IL-1 $\beta$ does not signal through STAT3 to induce IL-6, STAT3 siRNA was used in the OSM+ IL-1 $\beta$ co-treatment experiment. In the MCF7 cells, STAT3 siRNA did not affect IL-1 $\beta$-induced IL-6 levels (sFig 7). However, OSM-induced IL-6 was suppressed by STAT3 siRNA in MDA-MB-231 cells, and with the OSM+IL-1 $\beta$ cotreatment, IL-6 levels were lower compared to control siRNA levels. Additionally, caffeic acid phenyl ester (CAPE), a p65 inhibitor, suppressed OSM induction of IL-6 in MDA-MB-231 cells despite OSM's inability to activate p65 phosphorylation (sFig 8). This may suggest some type of crosstalk occurring between STAT3 and NFkB signaling pathways in MDA-MB-231 cells (59). Overall, these results indicate that there are minor differences between cell lines in terms of intracellular signaling activation in response to OSM and IL-1 $\beta$, and suggest that there may be a mechanism of IL- 6 suppression that is independent of phosphorylation in the ER+ cells.

ER interaction with STAT3 is suppressed by OSM and IL-1 $\beta$ in MCF7 cells

In other studies, ER has been known to directly interact with various signaling molecules including p65 to suppress downstream signaling despite its apparent activation by protein phosphorylation (60). To assess whether ER may be binding to intracellular signaling pathway proteins, an immunoprecipitation with ER pull-down was performed 
on ER+ MCF7 and T47D cell lysates treated with OSM and/or IL-1 $\beta$ for 48 hours. While no interaction between ER and p65, AKT, or ERK was observed (data not shown), a 50\% reduction in interaction between ER and STAT3 in response to OSM or IL-1 $\beta$ in MCF7 cells was seen using a STAT3 immumoblot of ERa-imunoprecipitation eluates (Fig 7E). With T47D cells, no interaction between ER and any of the signaling proteins was detected (data not shown). This suggests that the interaction of ER with STAT3 may suppress OSM induction of IL-6 in MCF7 cells, and that in the ER+ T47D cells an alternate mechanism may be operant. Collectively, OSM and IL-1 $\beta$ appear to induce IL-6 in a synergistic manner with OSM signaling through STAT3 and IL-1 $\beta$ operating through p65 and ERK.

\section{Discussion}

Previous therapeutic interventions against inflammatory cytokines such as IL-6 have failed in clinical trials despite their well-known role in tumor and metastasis promotion $(9,10,12)$. Nevertheless, there has been renewed interest in mitigating inflammation in the tumor microenvironment, as a growing recognition that chronic tumor inflammation leads to angiogenesis, immunosuppression, proliferation, and metastasis exists (61). Inflammatory cytokines such as OSM, IL-6 and IL-1 $\beta$ have individually been shown to promote effects associated with metastatic cancer $(9,29,62$, 63). Here we show for the first time the interplay between the three cytokines in breast cancer. We demonstrate that not only were the levels of tumor and stromal expression of these cytokines correlated with metastatic disease and decreased patient survival, but that there was an ER subtype-specific response in terms of OSM- and or IL-1 $\beta$-mediated induction of IL-6. Specifically, ER+ MCF7 and T47D human breast cancer cells failed to 
produce IL-6 in response to OSM, while high levels of IL-6 was produced with OSM treatment in the ER- MDA-MB-231 cells. Other studies have shown that there are subtype-specific differences in breast cancer's response to IL-6 cytokines where the magnitude of STAT3 pathway activation in response to IL-6 correlated to ER expression $(64,65)$. Additionally, our results indicated that, OSM and IL- $1 \beta$ synergistically increased IL-6 production by activating two separate pathways, STAT3 and NFאB, in ER-MDA-MB-231 cells. This was also seen in the ER+ MCF7 cells, albeit at a much lower level. Studies on arthritis and joint physiology have also demonstrated a synergistic relationship between OSM and IL-1 $\beta$ in terms of joint damage and synovial fibroblastmediated inflammation $(25,56)$. We further showed that OSM induces IL-6 secretion in the MDA-MB-231 cells through the STAT3 pathway, and that ER appears to inhibit downstream STAT3 signaling (Fig. 8). Unlike in the ER+ MCF7 cells, which produced IL-6 in response to IL- $1 \beta$ and OSM co-treatment, T47D cells did not produce IL-6 under any conditions tested. This suggests that despite these two cell lines being the same luminal A breast cancer cell subtype, there are major differences in their intracellular signaling mechanisms. Additional studies are required to elucidate this difference as there may be mechanisms independent of pathway activation via phosphorylation. Taken together, this study highlights the complex interplay of various inflammatory cytokines in a manner that is subtype specific.

Of the three cytokines studied in the paper, only therapeutic interventions targeting IL-6 have received a lot of attention $(9,47,66-68)$. Recent clinical trials using an anti-IL-6 biologic, siltuximab, have failed to produce any clinically positive results in the treatment of solid tumors $(10,11)$. Our results suggest that inflammation in the tumor 
microenvironment may be instigated by multiple cytokines and that IL- 6 may only be a part of the whole picture. Here, we have shown that OSM and IL-6 are correlated with each other in patient serum data and in an in vivo mouse model of breast cancer (manuscript in preparation). Additionally, we show that OSM, IL-6, and IL-1 $\beta$ levels are correlated with each other in patient data, and that high levels of these cytokines lead to reduced patient survival. To date, there are no anti-OSM therapeutics available, nor are there any clinical trials in progress or planned to investigate anti-OSM therapies against solid tumors. On the other hand, there are several anti-IL-1 $\beta$ (Anakinra) therapeutics available that were developed for Castleman's disease, where abnormally high levels of inflammatory cytokines are produced by various immune cells $(69,70)$. While this antiIL-1 $\beta$ therapeutics appeared promising for the treatment metastatic disease (35), it failed to produce significantly positive results in a colorectal cancer model (36). Additional studies and clinical trials for anti- IL-1 $\beta$ therapeutics are underway to assess their antitumor capacity in other types of metastatic disease $(37,71,72)$.

An interesting aspect of this study is that we demonstrated a breast cancer subtype-specific difference in the production of IL- 6 in response to OSM and/or IL-1 $\beta$, thus suggesting that subtype-specific markers like ER may play a role in this difference. Previous studies have shown that induction of IL-6 expression is dependent on the NFKB pathway and that ER suppresses this signaling through inhibition of p65 $(60,73)$. However, our immunoprecipitation results suggest that ER may interact with STAT3 to suppress OSM induced-IL-6 secretion. Furthermore, suppression of the STAT3 pathway by siRNA also reduced OSM-induced IL-6 production to untreated control levels in MDA-MB-231 cells. On the other hand, induction of IL- 6 by IL- $1 \beta$ alone or by both 
OSM and IL-1 $\beta$ was not affected by STAT3 siRNA. Instead, IL-1 $\beta$ has been previously shown to induce IL-6 expression through p65 and PI3K dependent pathways (57). Here, we show that IL-1 $\beta$ induction of IL-6 is partially dependent on ERK signaling in MCF7 cells, as the usage of the ERK inhibitor suppressed IL-1 $\beta$ and IL- $1 \beta+$ OSM induction of IL-6.

Our immunoprecipitation result in MCF7 cells also suggested that treatment of the cells with OSM, IL-1 $\beta$, or with both cytokines reduced the association of ER with STAT3. Initially this seems to be an unusual result as IL-1 $\beta$ does not utilize the STAT3 pathway to induce downstream signaling. However there appears to be some measure of cross talk between the STAT3 pathway and the p65 pathway, where in some cases the function of the pathway may be interdependent on one another (74). We also saw reduction in OSM induced IL-6 secretion from MDA-MB-231 cells with the use of the NFאB inhibitor caffeic acid phenyl ester (CAPE) (75), despite OSM's inability to activate p65 phosphorylation. This suggests that there may be crosstalk between STAT3 and $\mathrm{NF} \kappa \mathrm{B}$ for the induction of IL-6, and may explain the synergistic relationship between OSM and IL-1 $\beta$. While the exact nature of the crosstalk mechanism is not well known, it appears that NFאB and STAT3 signaling proteins must be activated and directly bind to each other during signaling $(59,74)$ (Fig. 8). However, if this is the case, STAT3 siRNA should have inhibited IL-1 $\beta$-mediated IL-6 secretion in MDA-MB-231 and MCF7 cells. A possible alternative explanation is that ER forms an inhibitory complex with STAT3 to suppress IL-6 expression, similar to the ER/p65 complex which is known to have a regulatory role in gene expression (76). This would make suppression of total STAT3 ineffective for reduction of IL-6 expression, as doing so would also render the ER- 
STAT3 inhibitory complex less effective at regulating IL-6 gene expression. While we have not been able to elucidate the exact mechanism of STAT3/ER interaction and the crosstalk with $\mathrm{NF \kappa B}$, these results necessitate investigation into pathways that are not necessarily canonically known to be activated by the specific cytokine. Therefore, further investigation into the mechanistic nature of how these signaling pathways interact with each other in the context of breast cancer is needed.

\section{Conclusions}

Collectively, our study demonstrates that OSM, IL-6, and IL-1 $\beta$ levels are correlated with each other and that cytokine signaling differs in an ER subtype-specific manner. This highlights the possible implications of multi-cytokine effects in the tumor microenvironment and that singular anti-cytokine therapies may not be sufficient for the successful treatment of metastatic breast cancer. This may be due to the fact that OSM, IL-6, and IL-1 $\beta$ each work independently to impair breast cancer patient survival and increase metrics of metastases. Thus, suppression of one cytokine may be countered by an increase in a different but related cytokine to maintain the cancer metastatic potential. Therefore, this study substantiates the rationale for a therapeutic design that simultaneously targets multiple cytokines, such as OSM, IL-6, and IL-1 $\beta$, as these cytokines are strongly intercorrelated in breast cancer. In conclusion, we suggest that further studies need to be done to unravel the complex interplay between these cytokines and that future clinical trials should include the inhibition of multiple cytokines. 


\section{Declarations}

Serum assessed for this chapter contain patient samples that were handled in accordance with the St. Luke's Medical Center (12-0298) and Boise State University (006MED15-006) Institutional Review Boards.

All animal experiments were approved by and performed in accordance with the animal guidelines of the Boise Veterans Affairs Medical Center (\#JOR0013-1) Institutional Animal Care and Use Committees. ' 


\section{Chapter Four: Figures}
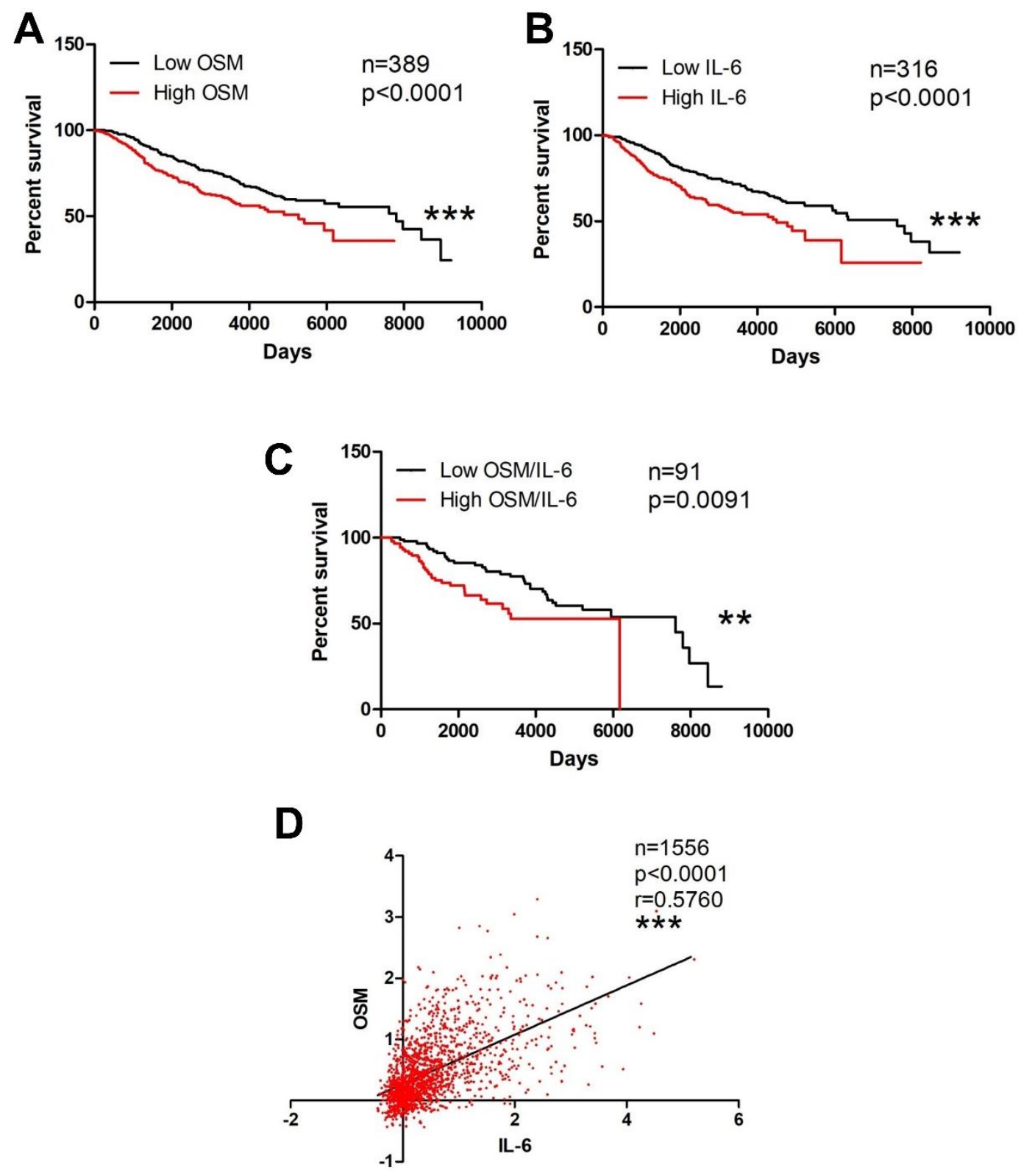

Figure 4.1. OSM and IL-6 are associated with decreased invasive breast cancer survival 
Figure 4.1. OSM and IL-6 are associated with decreased invasive breast cancer survival.

A, Kaplan-Meier curves of invasive breast cancer patient samples with high OSM expression levels show significant reduction in survival compared to curves of patients with low OSM expression levels. Log-rank test $(\mathrm{p}<0.0001) \mathbf{B}$, This trend is repeated with IL-6. Survival curves of breast cancer patients with high IL-6 expression have reduction in survival compared to patients with low IL-6 expression level Log-rank test $(\mathrm{p}<0.0001)$. C, Survival curves of breast cancer patients with high co-expression of OSM and IL-6 also demonstrate a reduction in survival compared to patients with low co-expression of both OSM and IL-6 Log-rank test $(\mathrm{p}=0.0091)$. D, Two-way correlation analysis depicts a statistically significant correlation between OSM and IL-6 expression levels in breast cancer patients with a Spearman coefficient of 0.576. $(\mathrm{p}<0.0001)$. 


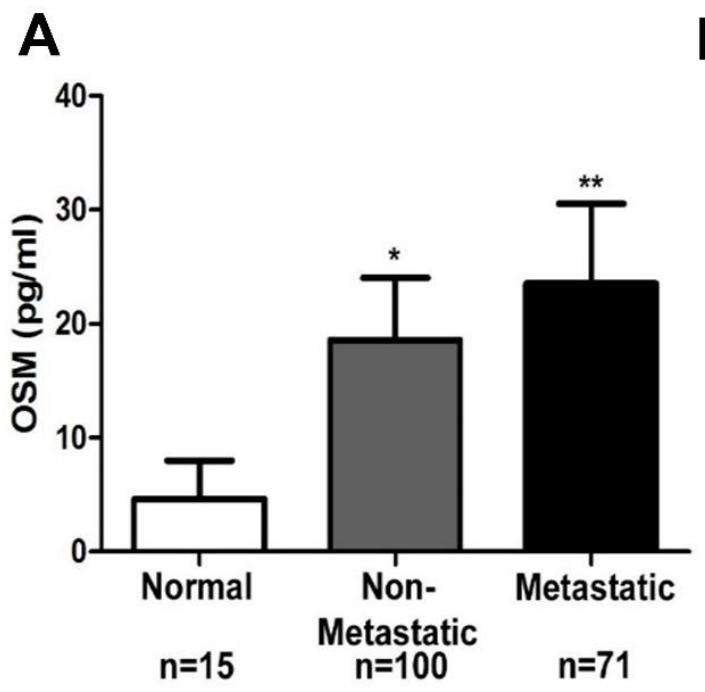

B
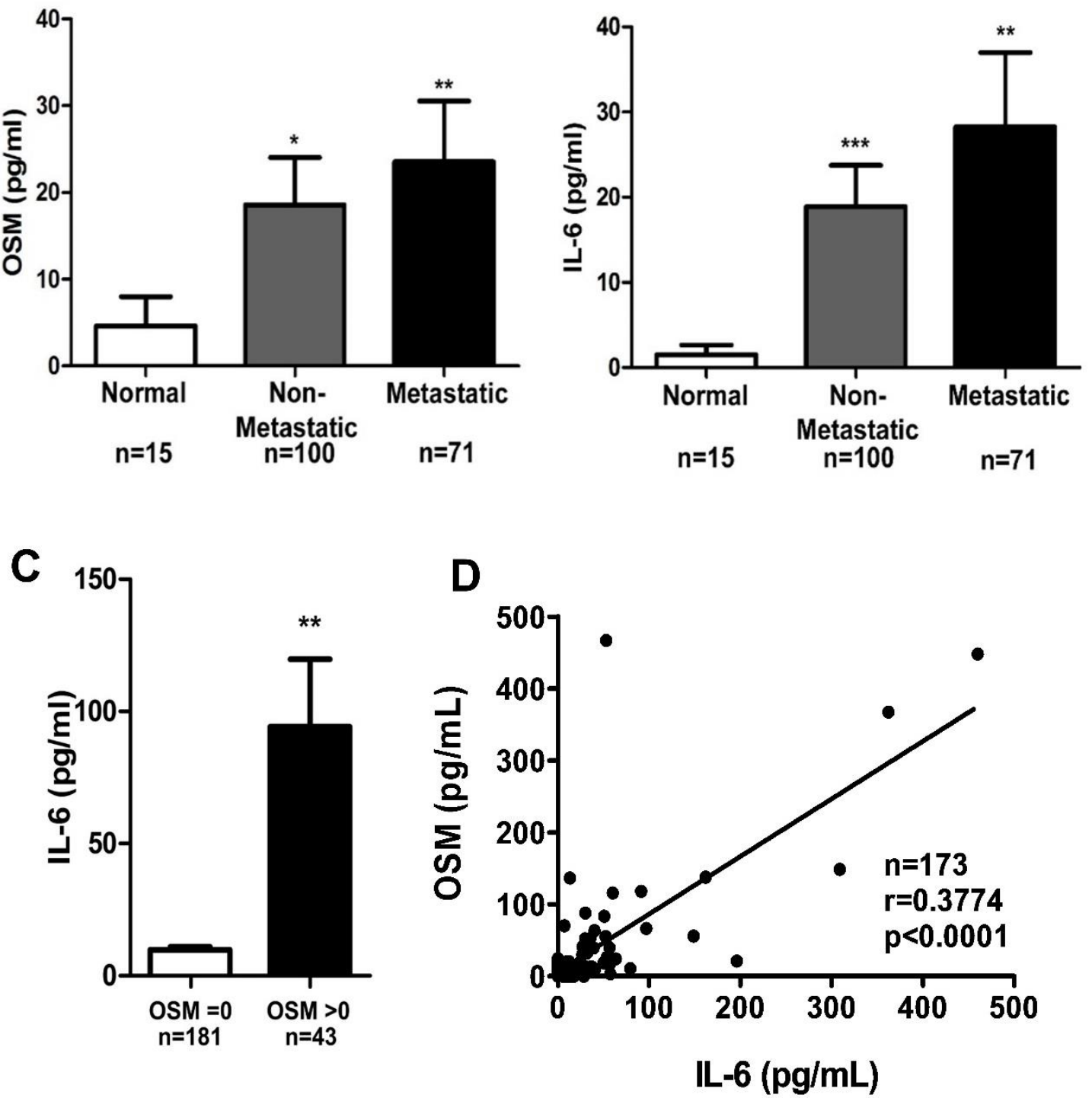

Figure 4.2. OSM breast cancer patient serum levels correlate with IL-6 levels

Figure 4. 2. OSM breast cancer patient serum levels correlate with IL-6 levels.

Sera from breast cancer patients were procured from various sources, and OSM levels (A) and IL-6 levels (B) were measured by ELISA. When OSM and IL-6 were assessed between normal patients versus patients with non-metastatic or metastatic breast cancer, there was a significant increase in serum OSM and IL-6 levels in both patients 
with non-metastatic or metastatic breast cancer versus normal patients. C, Patient sera with undetectable OSM levels also have low IL-6 levels $(8.5 \mathrm{pg} / \mathrm{ml})$, while patient sera with detectable levels of OSM have high levels of IL-6 (89 pg/ml). D, The serum cytokines concentration data was then assessed for correlation and suggests that higher levels of serum OSM correlates with higher levels of serum IL-6 (Spearman Correlation coefficient $=0.923(95 \%$ CI $0.825,1.0) \mathrm{P}<0.0001)$. Data expressed as mean $+/-$ SEM. and assessed by one-way ANOVA with Tukey's post-test*p $<0.05$, **p<0.01, $* * * p<0.001$. 
A

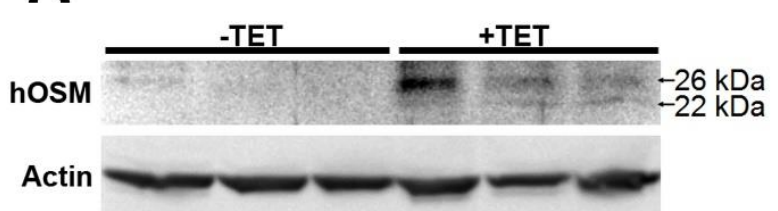

B
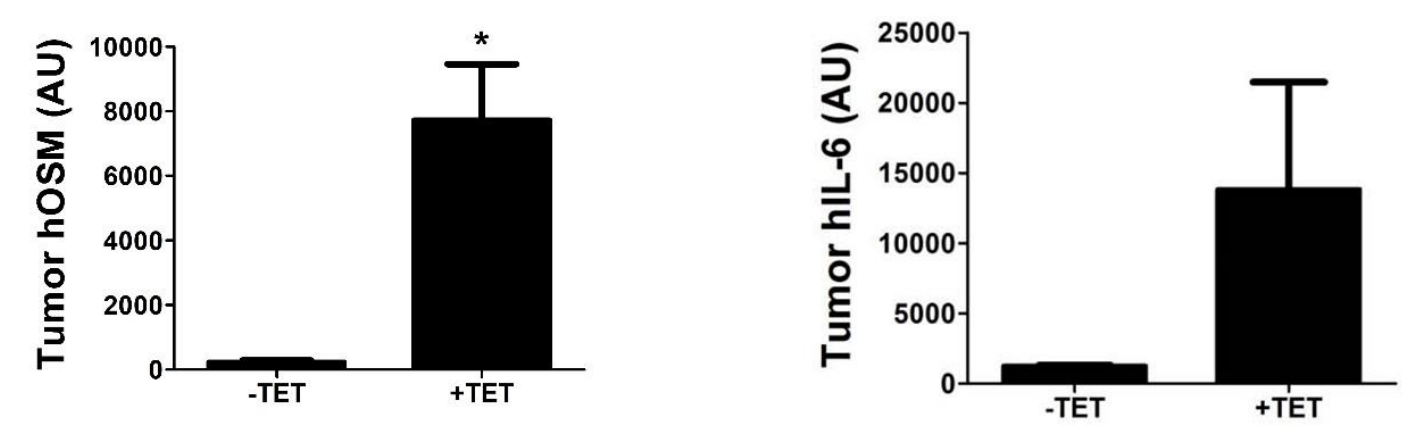

C

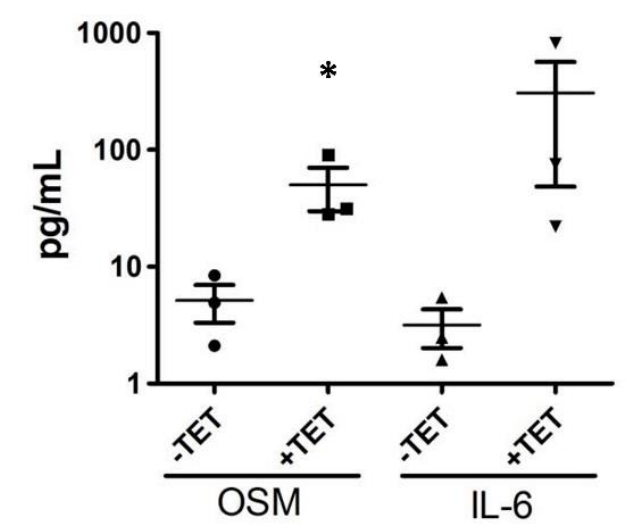

Figure 4.3. OSM induces IL-6 in an animal model of human breast cancer 
Figure 4.3. OSM induces IL-6 in an animal model of human breast cancer.

A,.MDA ${ }^{\mathrm{TO} / \mathrm{OSM}}$ cells were injected orthotopically in vivo in athymic nude mice. After the tumors became palpable, the animals were given tetracycline, and after one week, the animals were sacrificed and tumors harvested. Western blot analysis of the tumors indicates that OSM levels increase in response to tetracycline administration (top). Densitometry of western blots indicate a 40-fold increase in tumor OSM in animals given tetracycline compared to animals given control water (bottom). B, IL-6 levels in the tumor, as assessed by western blot, also show similarly elevated levels in the tetracycline-treated animals. C, Sera collected from the animals were assessed for hOSM and hIL-6 levels by ELISA. Animals given tetracycline have an $~ 10$-fold increase in mean serum hOSM levels and an 100-fold increase in mean serum hIL-6 levels. Data expressed as mean +/- SEM, and significance assessed by two-tailed student's t-test. $* \mathrm{p}<0.05$. 

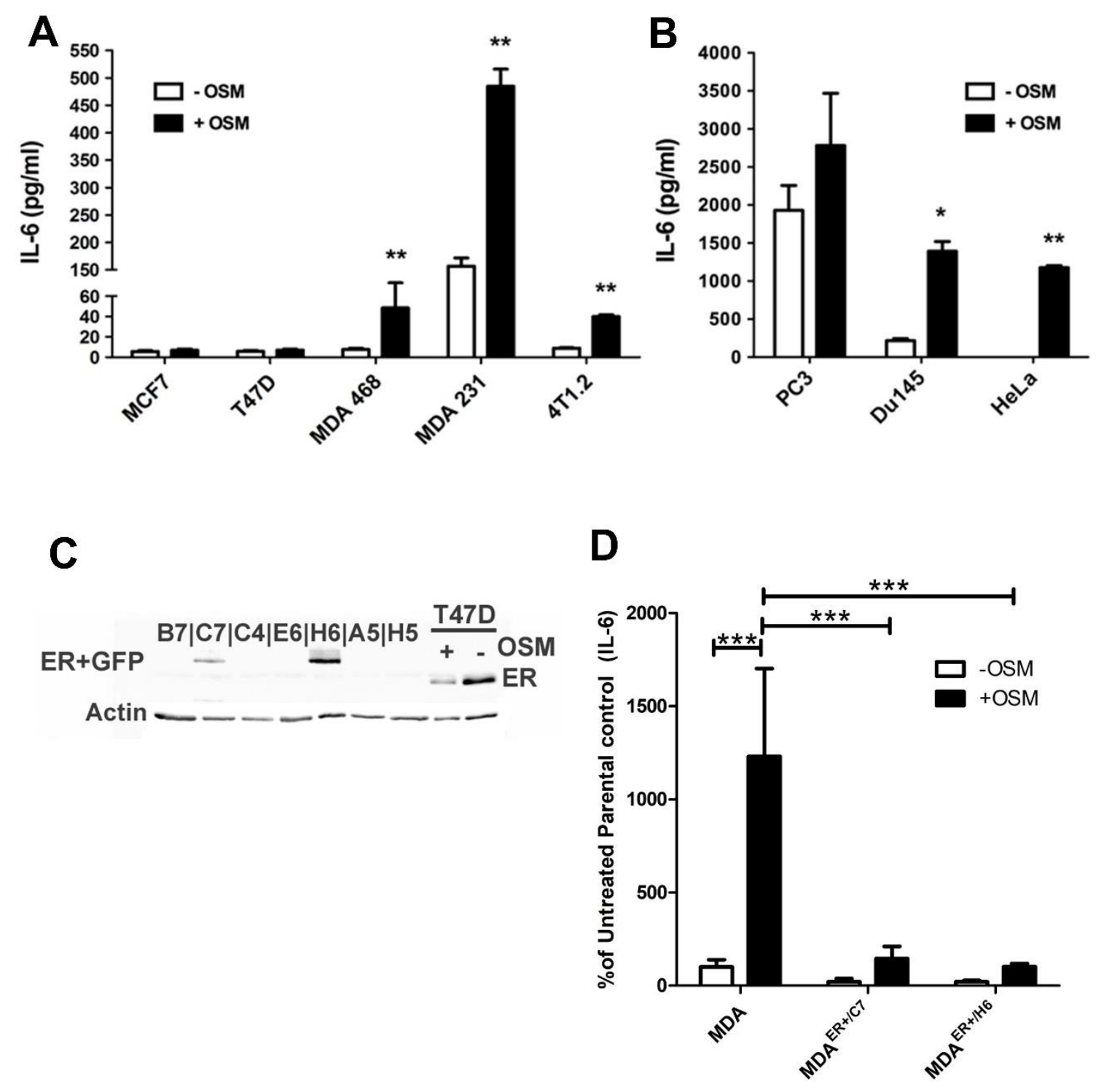

Figure 4.4. OSM induces IL-6 secretion in an ER-dependent manner 
Figure 4.4. OSM induces IL-6 secretion in an ER-dependent manner.

A, IL-6 secretion levels were measured by ELISA on CM collected from various OSM-treated cells. ER-MDA-MB-231, MDA-MB-468, and 4T1.2 cells display high (over 4-fold) levels of OSM-induced IL-6 secretion, while ER+ MCF7, and T47D cells do not. B, OSM also induces IL-6 secretion in PC3 and DU145 (prostate) and HeLa (ovarian) ER- non-breast cancer cell types. C, MDA-MB-231 cells were transfected with an ER $\alpha$ expression vector, and the presence of ER $\alpha$ in transfected colonies was determined by western blot. The two ER expressing cell lines are designated as $\mathrm{MDA}^{\mathrm{ER}+/ \mathrm{C} 7}$ and MDA ${ }^{\mathrm{ER}+/ \mathrm{H} 6}$. D, $\mathrm{MDA}^{\mathrm{ER}+/ \mathrm{C} 7}$ cells secrete 9-fold less IL-6 and MDA ${ }^{\mathrm{ER}+/ \mathrm{H} 6}$ cells secrete 12-fold less IL-6 in response to OSM compared to the parental MDA-MB231 cells. Data expressed as mean \pm SEM and significance assessed by one-way ANOVA with Tukey's post-test. ${ }^{*} \mathrm{p}<0.05, * * \mathrm{p}<0.01, * * * \mathrm{p}<0.001$. 
A

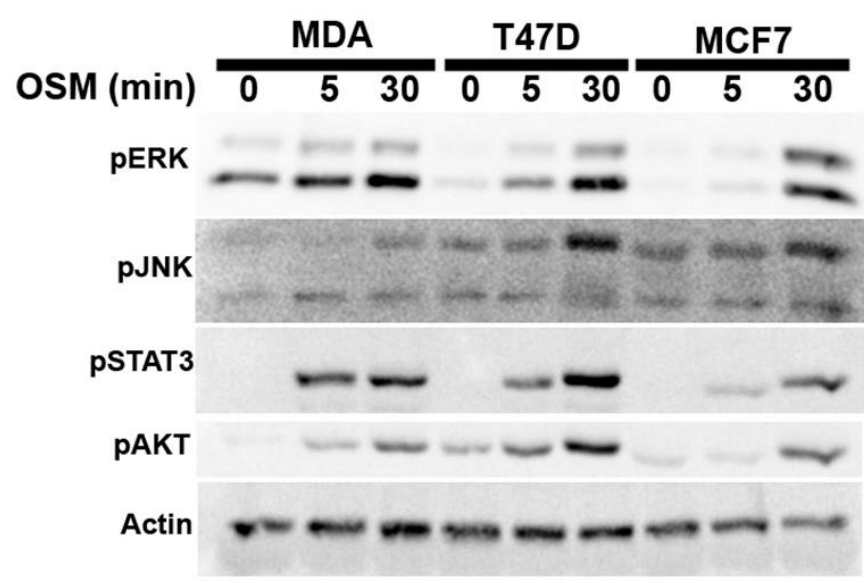

B

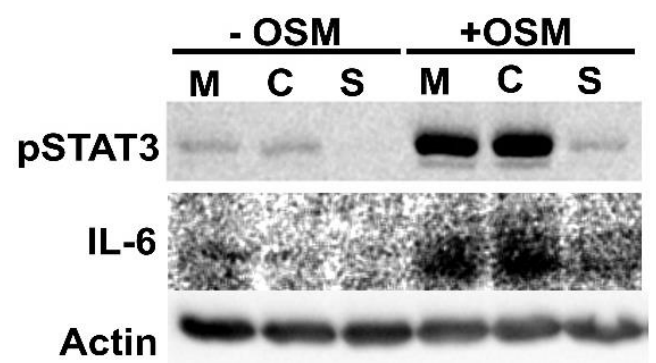

$M=$ Mock $\quad C=C$ trl siRNA $S=S T A T 3$ siRNA

C

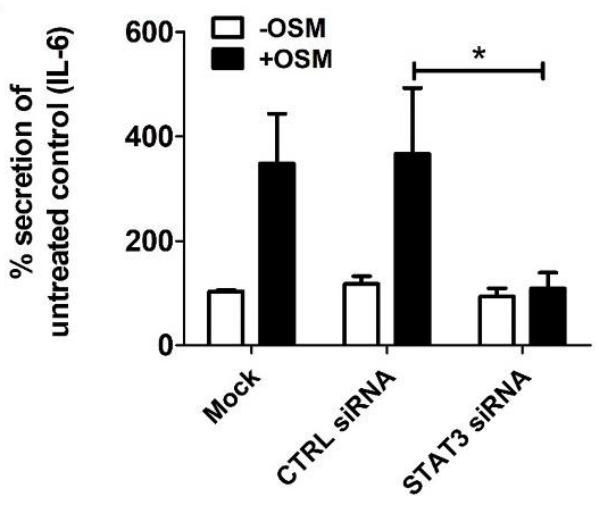

Figure 4.5. OSM signals through STAT3 in ER-MDA-MB-231 cells to induce IL- 
Figure 4.5. OSM signals through STAT3 in ER- MDA-MB-231 cells to induce IL-6.

A, OSM induces the phosphorylation of multiple signaling pathways including ERK, JNK, AKT, and STAT3 as assessed by western blot. Overall, phosphorylation of STAT3 and ERK occurs faster than other signaling pathway proteins. B, With STAT3 suppression by STAT3 siRNA, there is little induction of Y705 phosphorylation of STAT3 by OSM. Additionally, OSM-induced IL-6, as detected by western blot, is also suppressed. C, STAT3 siRNA suppressed OSM induction of IL-6 production as assessed by ELISA in MDA-MB-231 cells. Data expressed as mean \pm SEM, and significance assessed by one-way ANOVA with Tukey's post-test. *p<0.05. 
A

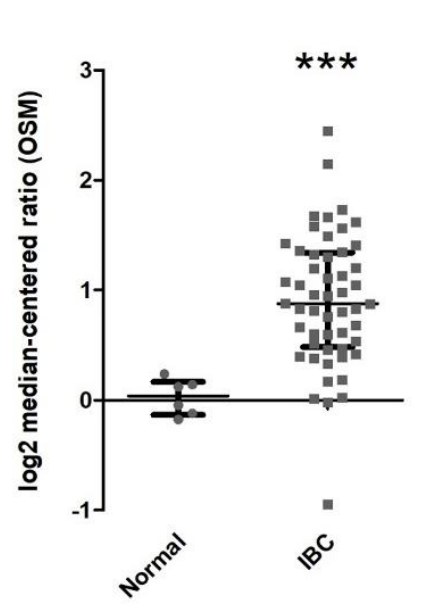

C
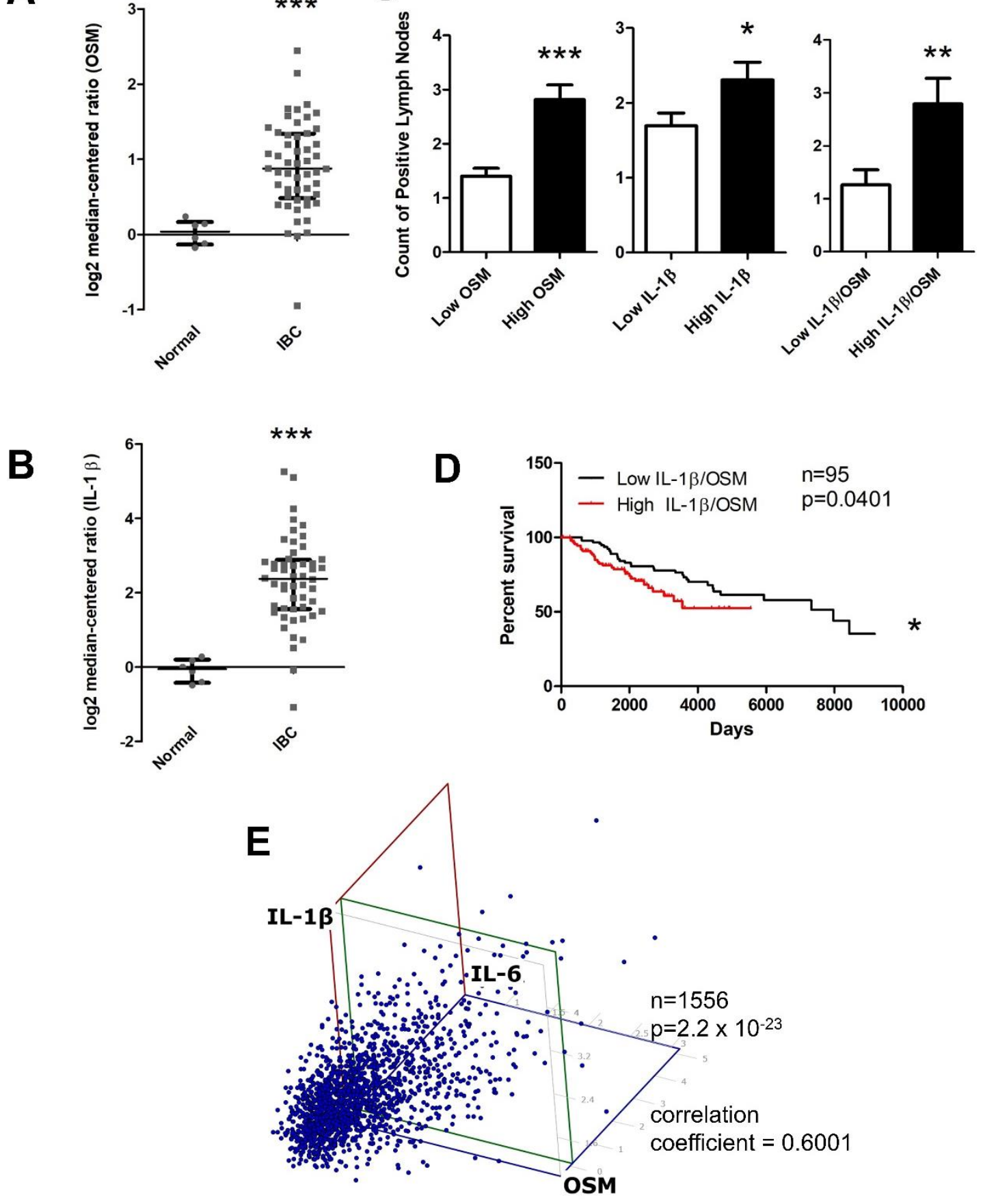

Figure 4.6. OSM and IL-1 $\beta$ expression is higher in invasive breast cancer compared to normal tissue and correlates with higher lymph node metastasis, decreased survival, and IL-6 levels 
Figure 4.6. OSM and IL-1 $\beta$ expression is higher in invasive breast cancer compared to $\underline{\text { normal tissue and correlates with higher lymph node metastasis, decreased survival, and }}$ IL-6 levels.

A, Using the FINAK dataset obtained from Oncomine ${ }^{\mathrm{TM}}$ we assessed stromal tissue expression of OSM and IL-1 $\beta$. Stromal tissue expression of OSM is 5.9-fold higher in invasive breast cancer patients compared to normal patients. B, Similarly, expression of IL-1 $\beta$ is 5.4 -fold higher in the stromal tissue of invasive breast cancer patients compared to normal patients. C, Using the Curtis dataset obtained from Oncomine ${ }^{\mathrm{TM}}$, we correlated OSM and IL-1 $\beta$ tissue expression levels to the number of lymph node metastases. Patients with high OSM (Left), high IL-1 $\beta$ (Center), and high co-expression of both OSM and IL-1 $\beta$ (Right) have significantly higher number of lymph node metastatic nodules compared to the respective low expression group. D, High coexpression of both OSM and IL-1 $\beta$ leads to a decreased overall patient survival. Log-rank test $(\mathrm{p}=0.0401)$. E, Expression of OSM, IL-1 $\beta$, and IL-6 were analyzed in a three-way correlation analysis with OSM on the $\mathrm{x}$-axis, IL- 6 on the $\mathrm{y}$-axis, and IL- $1 \beta$ on the z-axis. There is significant correlation with a coefficient of 0.6001 , with a p-value of $2.2 \times 10^{-23}$. Bar and scatter plot data expressed as mean \pm SEM, and significance assessed by twotailed student's t-test. $* \mathrm{p}<0.05, * * \mathrm{p}<0.01, * * * \mathrm{p}<0.001$ 


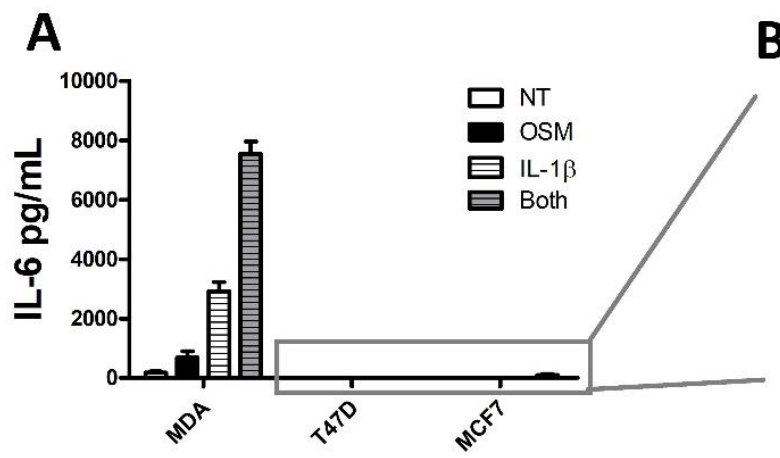

C

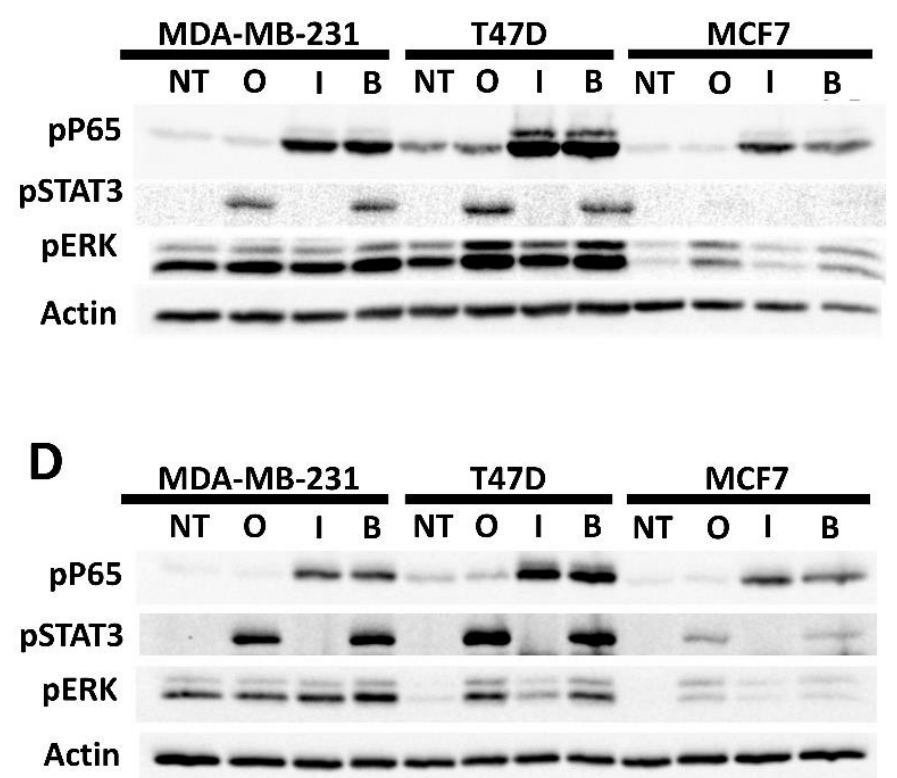

B

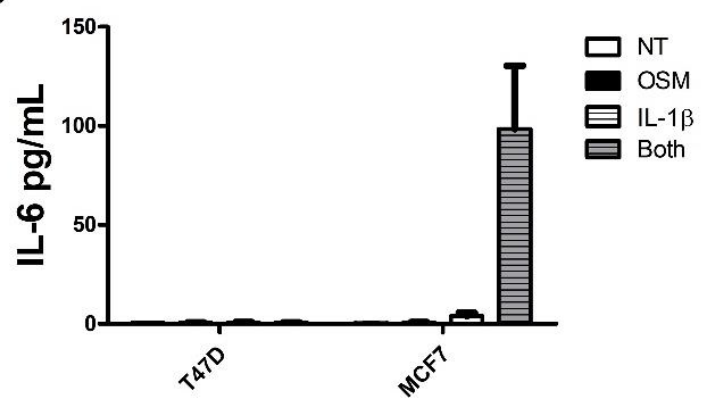

E

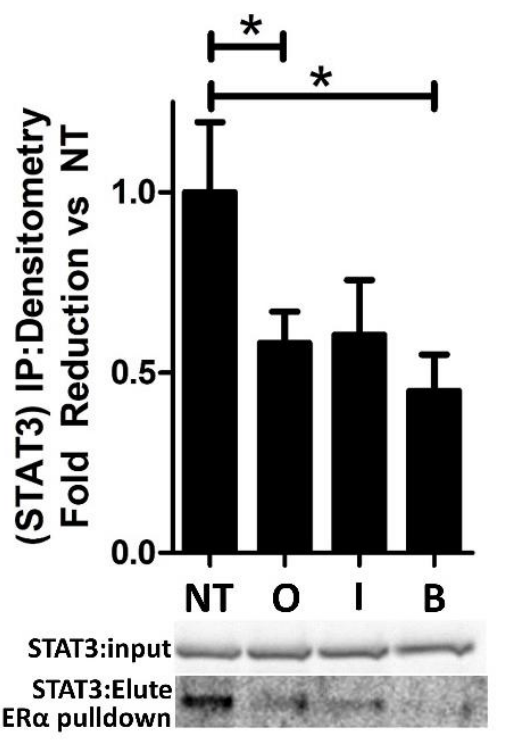

$$
\begin{aligned}
& \text { NT }=\text { No treatment } \quad I=10 \mathrm{ng} / \mathrm{mL} \text { IL-1 } \beta \\
& O=10 \mathrm{ng} / \mathrm{mL} \text { OSM } B=\text { Both at } 10 \mathrm{ng} / \mathrm{mL} \text { each }
\end{aligned}
$$

Figure 4.7. OSM and IL-1 $\beta$ activate separate signaling pathways and synergistically induce IL-6 secretion 
Figure 4.7. OSM and IL-1 $\beta$ activate separate signaling pathways and synergistically induce IL-6 secretion.

A, IL-1 $\beta$ alone promotes IL-6 production in MDA-MB-231 and MCF7 cells, while a combination of OSM and IL-1 $\beta$ causes a synergistic response in IL-6 secretion. T47D cells do not produce IL-6 in any of these conditions. B, Reduced IL-6 scale to allow visualization of MCF7 cell-IL-6 induction. C, With a 20-minute cytokine treatment with OSM, phosphorylation of STAT3 but not p65 is upregulated, while ERK is moderately phosphorylated. MCF7 cells have a weak STAT3 induction in response to OSM. A 20-minute cytokine treatment with IL-1 $\beta$ on the other hand induces the phosphorylation of p65 but not STAT3. D, The above phosphorylation of STAT3 and p65 are sustained at 72 hours with the same cytokine treatments. E, MCF7 cells were treated with OSM and/or IL-1 $\beta$ for 48 hours, and the cell lysates were run through an immunoprecipitation with an ER pulldown. The eluate was then immunoblotted with the input for STAT3. Lysates collected from MCF7 cells treated with OSM or with both cytokines have significantly reduced ER-STAT3 interaction. Data expressed as mean \pm SEM, and significance assessed by one-way ANOVA with Tukey's post-test. * $\mathrm{p}<0.05$, 


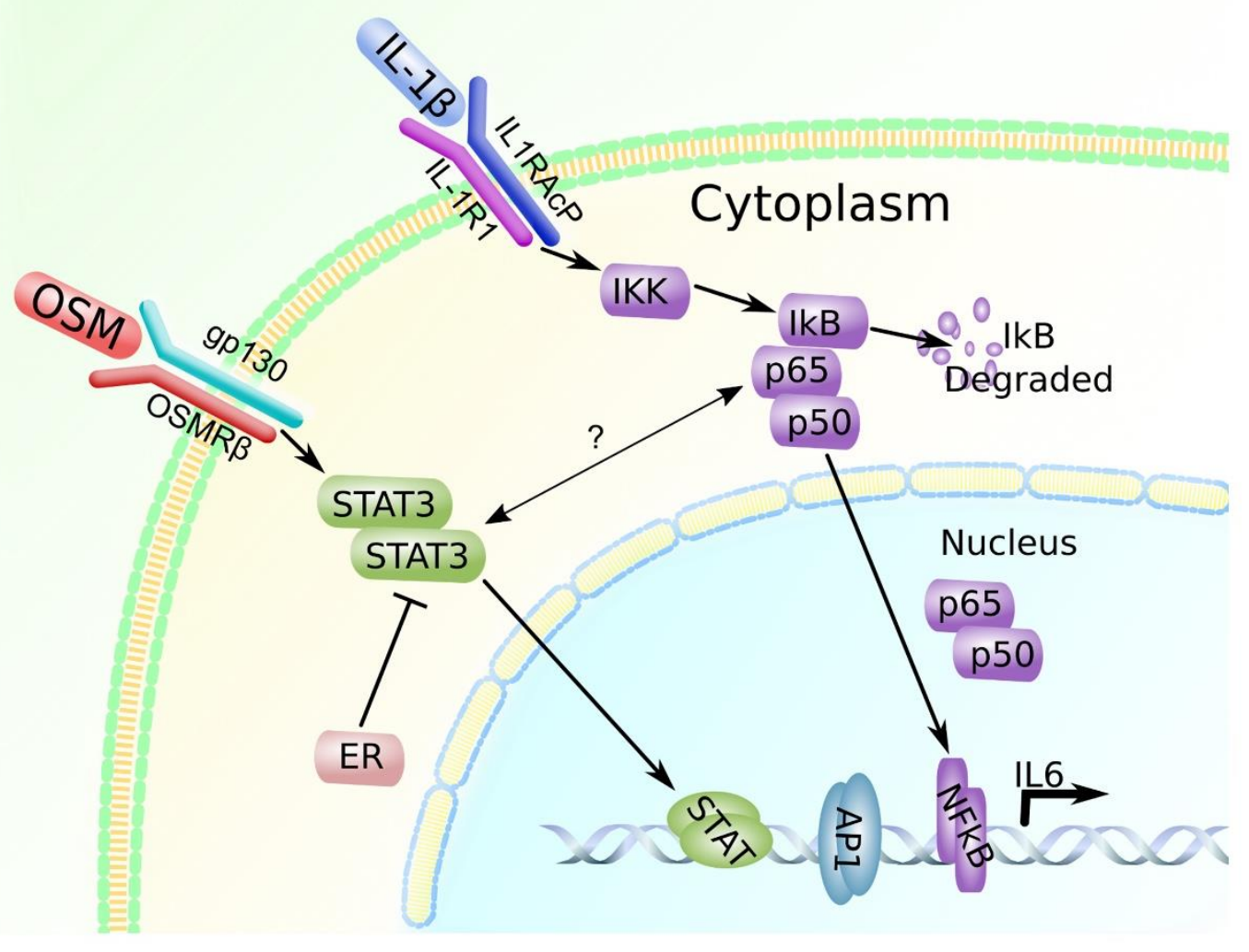

Figure 4.8. OSM and IL-1 $\beta$ promote IL-6 expression in a breast cancer cellsubtype specific manner

Figure 4.8. OSM and IL-1 $\beta$ promote IL-6 expression in a breast cancer cell-subtype specific manner.

OSM signals through the STAT3 pathway and leads to IL-6 induction in MDAMB-231 cells, while IL-1 $\beta$ induces IL-6 through the p65 pathway. In MCF7 cells, ER may be interacting with STAT3 to suppress IL-6 production. There may also be some crosstalk-like interaction between STAT3 and p65, however the exact nature of this interaction is not known $(59,74)$. 


\section{Chapter Four: Supplemental Figures}

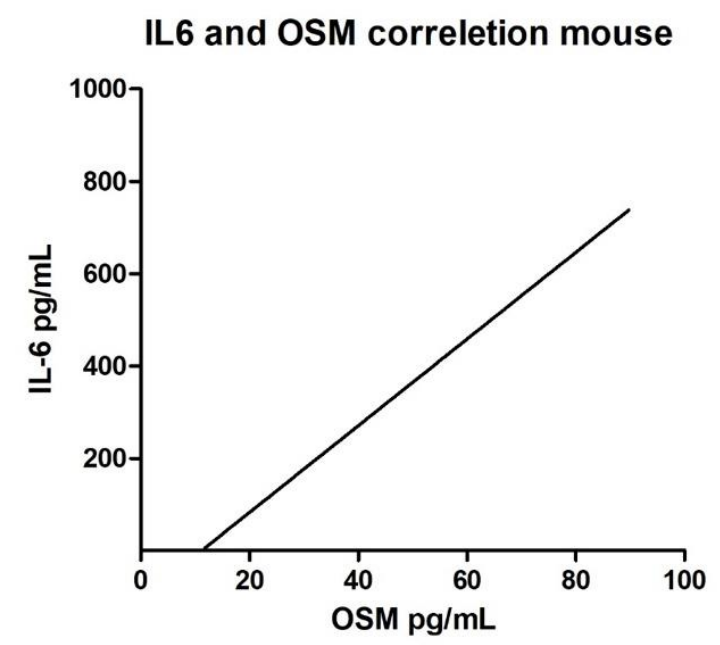

Spearman coefficient of $0.9058 \quad \mathbf{p}=\mathbf{0 . 0 0 3 4}$

\section{Figure 4.S1. OSM and IL-6 serum concentration is correlated in vivo}

Figure 4.S1. OSM and IL-6 serum concentration is correlated in vivo.

OSM and IL-6 serum concentrations in the MDA ${ }^{\text {TO/OSM }}$ mouse model were assessed by ELISA, and the resultant numbers were analyzed using a correlation analysis. OSM and IL-6 levels correlate with a spearman correlation coefficient of 0.9058 and a pvalue of 0.0034 . 


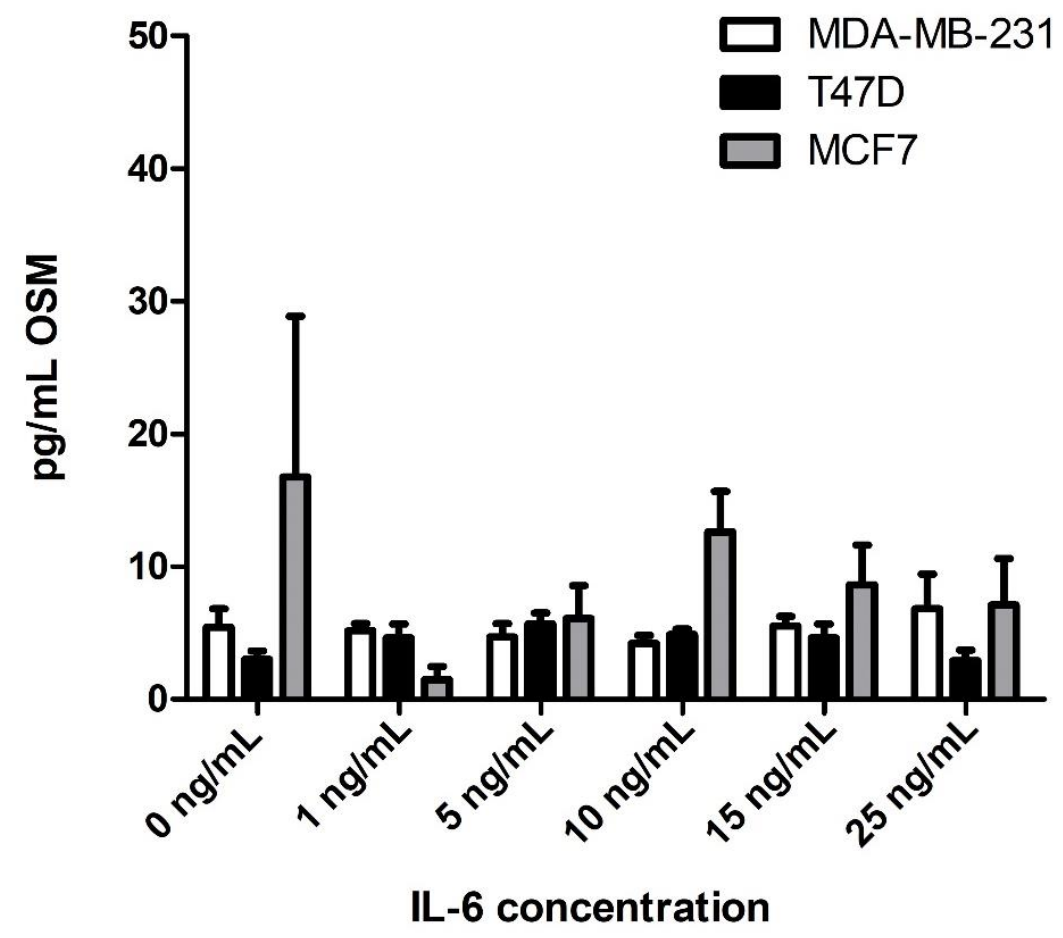

Figure 4.S2. IL-6 does not promote OSM secretion

Figure 4.S2. IL-6 does not promote OSM secretion.

MDA, T47D, and MCF7 cells were treated with IL-6, and secreted OSM levels in the conditioned media were assessed by ELISA. IL-6 did not promote OSM secretion in any of the cell lines tested. 


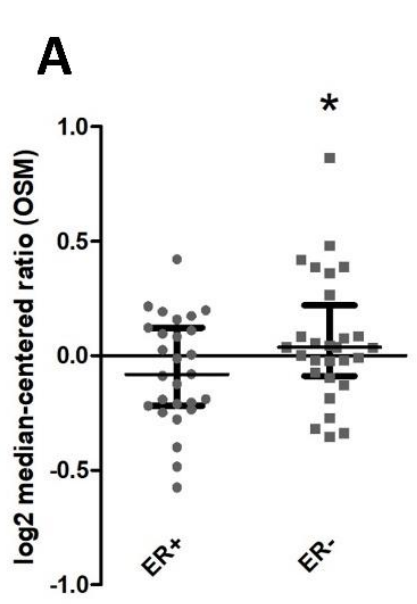

B

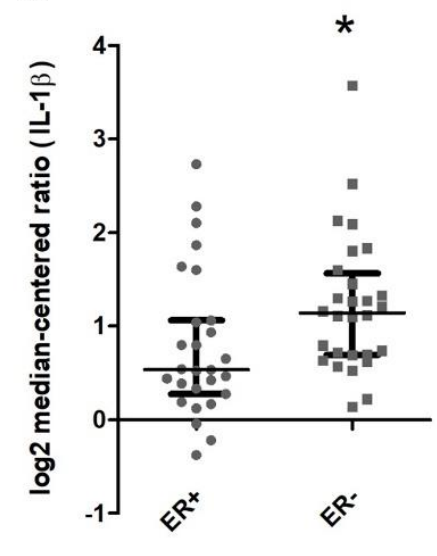

C

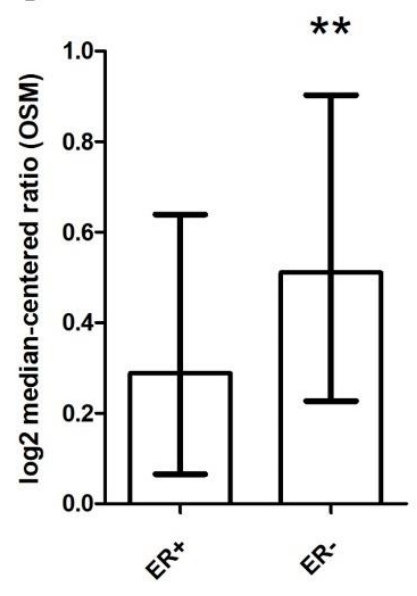

D

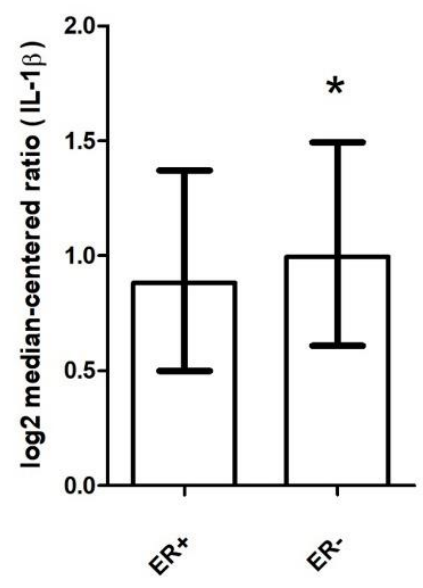

Figure 4.S3. IL-6 does not promote OSM secretion 
Figure 4.S3. Stromal and tumor tissue expression of both OSM and IL-1 $\beta$ are higher in ER-patients.

A, Stromal OSM and B, IL-1 $\beta$ expression is significantly higher in patients with ER- breast cancer compared to ER+ breast cancer, as assessed using the Finak Oncomine $^{\mathrm{TM}}$ dataset. Additionally, using the Curtis Oncomine ${ }^{\mathrm{TM}}$ dataset, tumor tissue expression of OSM (C) and IL-1 $\beta$ (D) is also significantly higher in ER- breast cancer patients compared to patients with ER+ breast cancer. Data expressed as $\log _{2}$ mediancentered ratio +/- SD and significance assessed by two-tailed student's t-test. $* \mathrm{p}<0.05$, $* * \mathrm{p}<0.01, * * * \mathrm{p}<0.001$ 

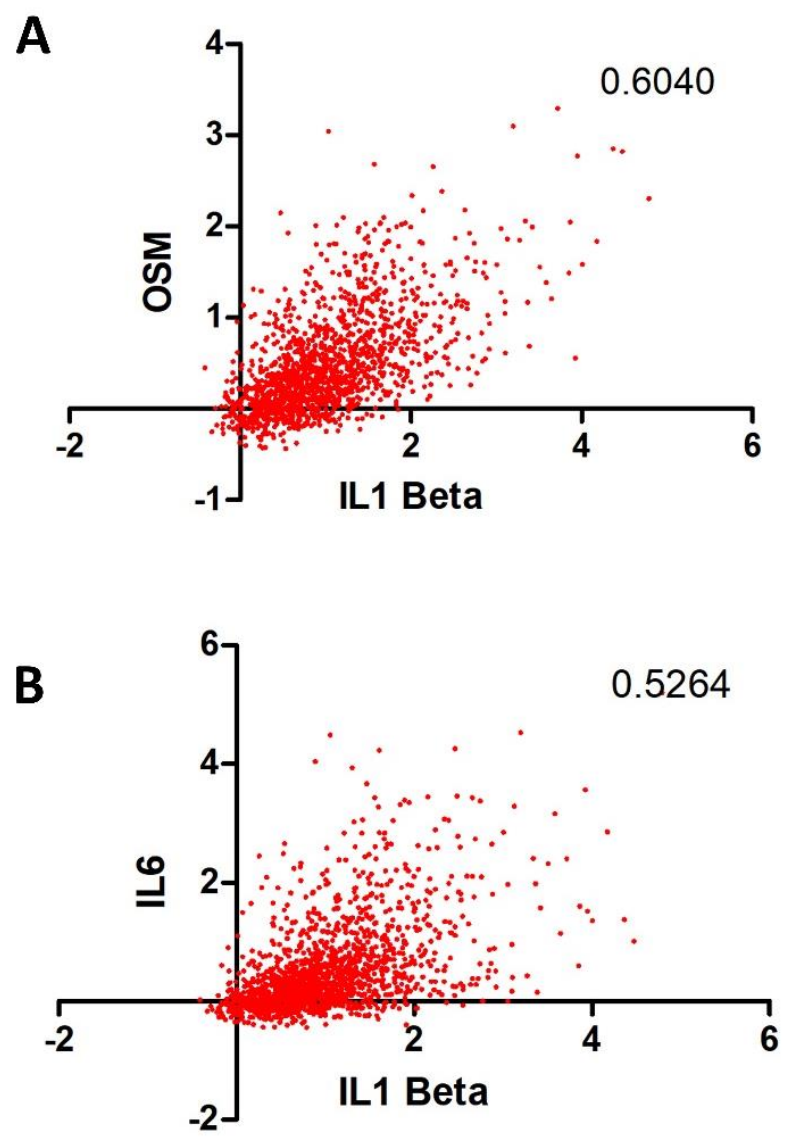

\section{Figure 4.S4. IL-1 $\beta$ mRNA expression levels correlate with both OSM and IL-6 in breast cancer patients}

Figure 4.S4. IL-1 $\beta$ mRNA expression levels correlate with both OSM and IL-6 in breast cancer patients.

The Curtis breast cancer dataset was assessed for OSM, IL-6, and IL-1 $\beta$ tumor mRNA expression levels. A, OSM and IL-1 $\beta$ expression levels correlates with each other with a spearman coefficient of 0.604 with a $p$ value of less than 0.0001 . B, IL-6 and IL$1 \beta$ expression levels correlates with each other with a spearman coefficient of 0.526 and a $\mathrm{p}$ value of less than 0.0001. Correlation coefficients were assessed using the Spearman correlation analysis. 


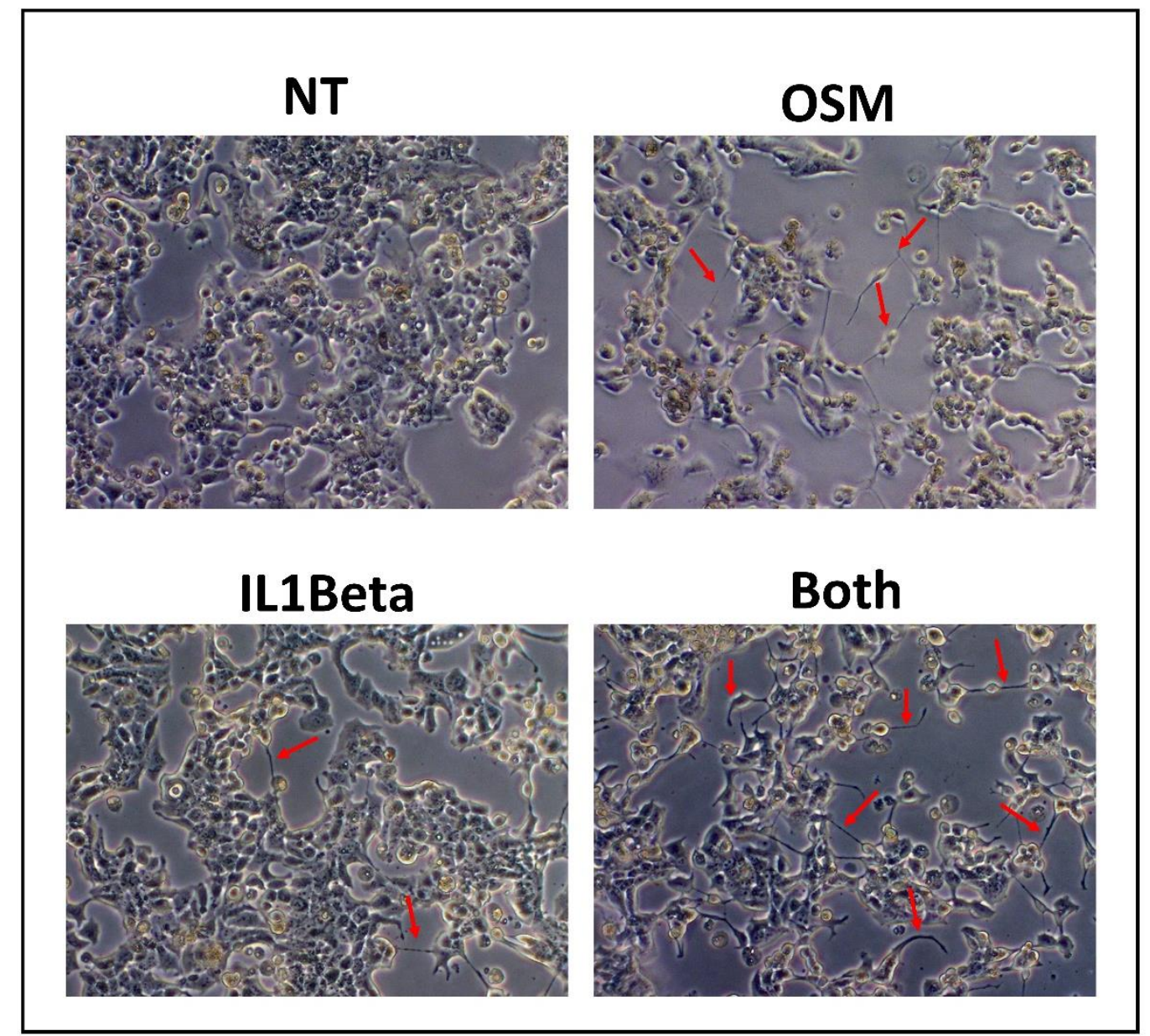

Figure 4.S5. OSM and IL-1 $\beta$ promotes EMT-like morphology in T47D cells

Figure 4.S5. OSM and IL-1 $\beta$ promotes EMT-like morphology in T47D cells.

T47D cells did not produce any IL-6 under any treatment conditions. To assess whether the cytokines were having any effect on T47D cells, they were treated with 10 $\mathrm{ng} / \mathrm{mL}$ of OSM and/or IL-1 $\beta$ for 72 hours and the cell morphology assessed by phase contrast microscopy. Treatment of the cells with OSM and/or IL- $1 \beta$ promotes an EMTlike cell morphology with the cells becoming more mesenchymal and spreading apart from each other. Red arrows indicate areas with invadopodium formation. These results indicate that OSM and IL-1 $\beta$ have an effect on T47D cells but does not produce IL-6 in response. 

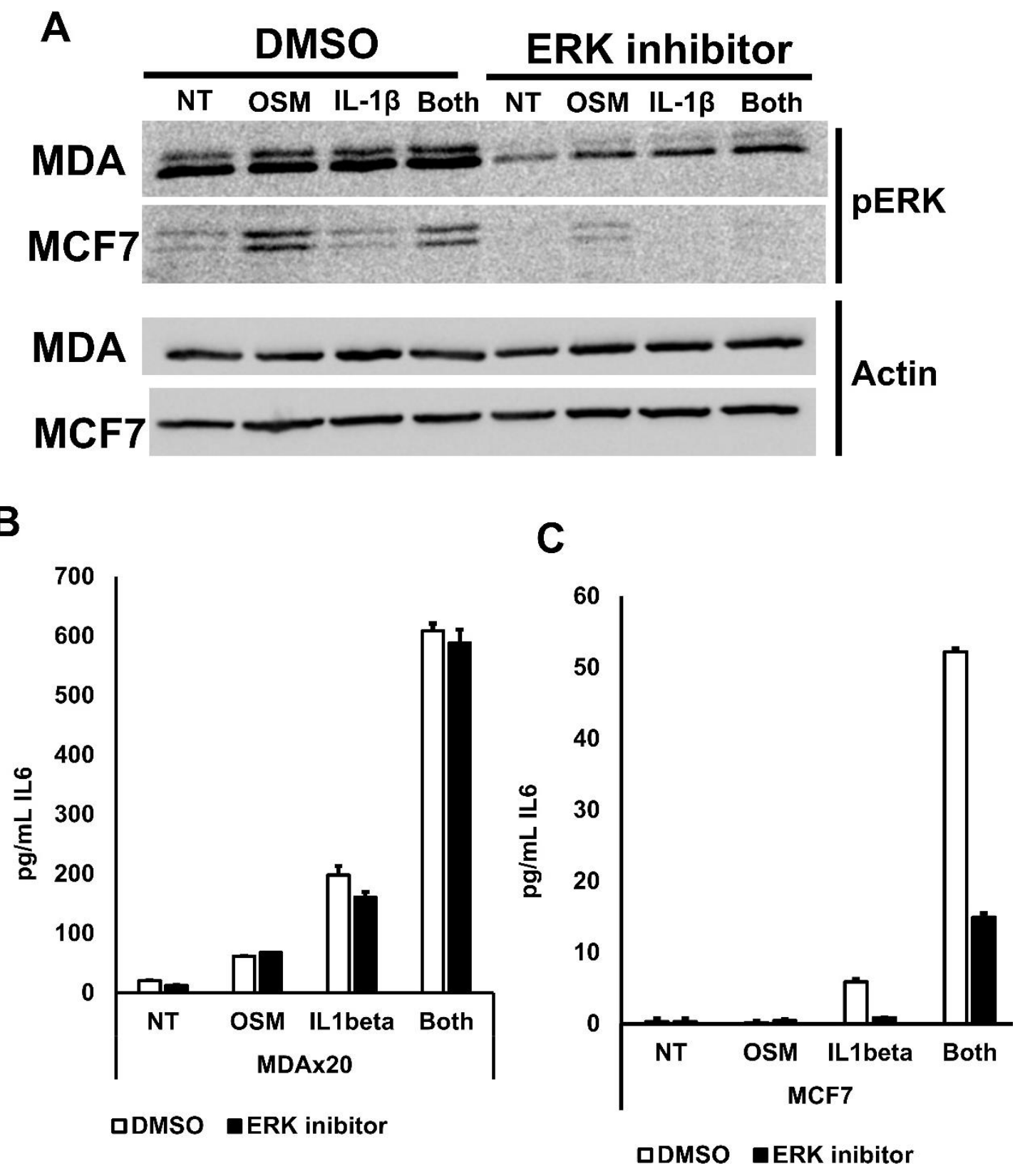

Figure 4.S6. ERK signaling is required for MCF7's synergistic response to OSM and IL-1 $\beta$ 
Figure 4.S6. ERK signaling is required for MCF7's synergistic response to OSM and IL-

$\underline{1 \beta}$.

MCF7 and MDA-MB-231 cells were treated with $10 \mathrm{ng} / \mathrm{mL}$ of OSM and/or IL-1 $\beta$ with or without an ERK inhibitor (50 $\mu \mathrm{M}, \mathrm{PD} 98059)$ or carrier (DMSO) for 48 hours. A, The ERK inhibitor suppresses pERK levels in both MCF7 and MDA-MB-231 cells. B, The ERK inhibitor suppresses IL-6 secretion in both IL-1 $\beta$ and OSM+ IL-1 $\beta$ treatment groups in MCF7 cells, but not in C, MDA-MB-231 cells. 


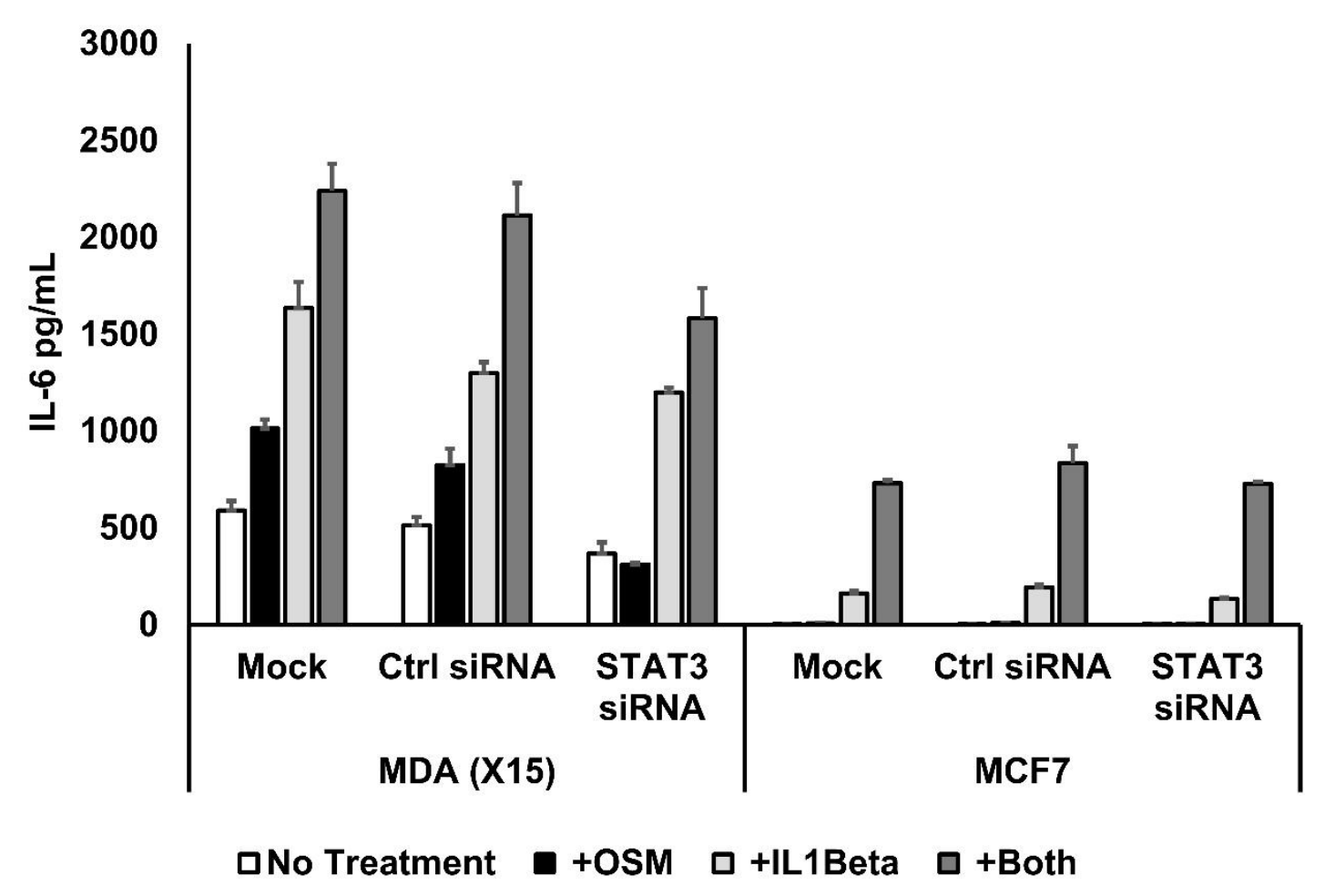

Figure 4.S7. STAT3 siRNA does not affect IL-1 $\beta$-induced IL-6 secretion

Figure 4.S7. STAT3 siRNA does not affect IL-1 $\beta$-induced IL-6 secretion.

MDA-MB-231 and MCF7 cells were treated with $25 \mathrm{nM}$ of STAT3 siRNA with or without $10 \mathrm{ng} / \mathrm{mL}$ of OSM and/or IL-1 $\beta$ for 72 hours. Although the usage of the STAT3 siRNA suppresses OSM-induced IL-6 secretion, it had no effect on IL-1 $\beta$ induced IL-6 secretion. 


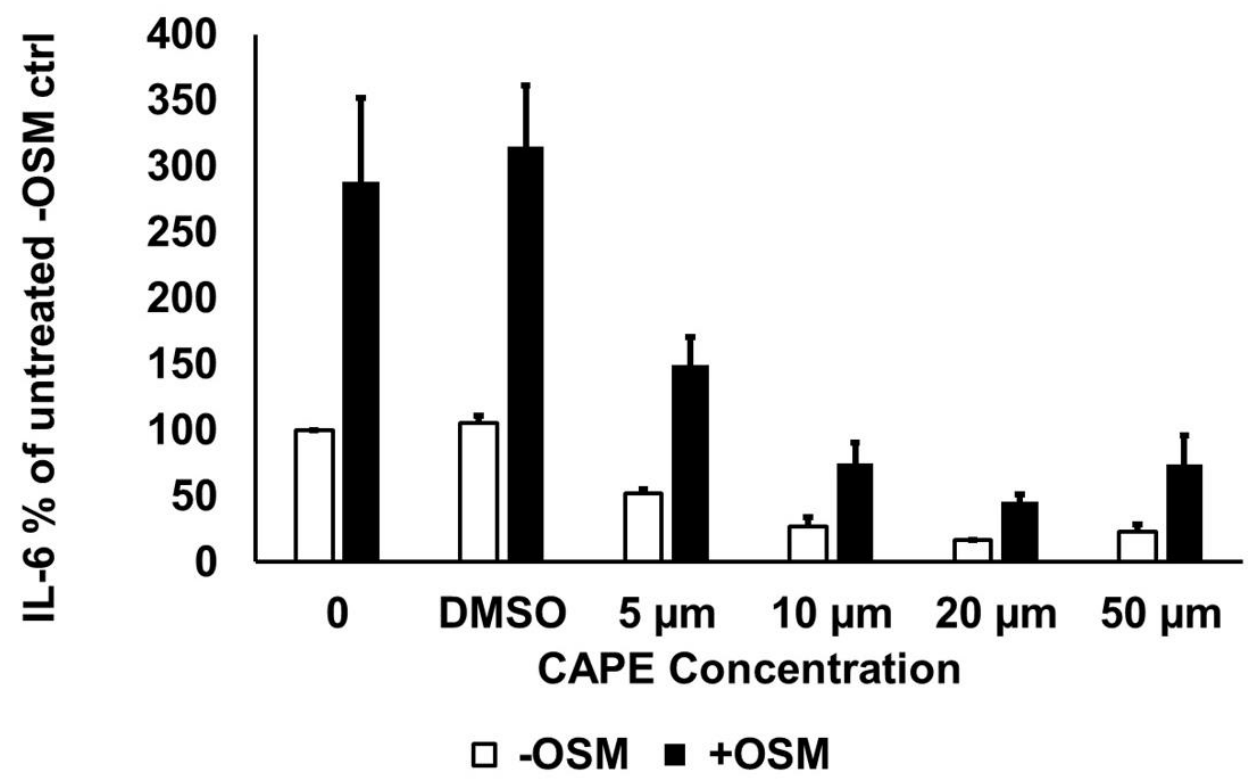

Figure 4.S8. CAPE inhibits OSM-induced IL-6 secretion

Figure 4.S8. CAPE inhibits OSM-induced IL-6 secretion.

MDA-MB-231 cells were treated with various concentrations of the p65 inhibitor CAPE with or without $25 \mathrm{ng} / \mathrm{mL}$ of OSM. By ELISA, CAPE inhibits OSM-induced IL-6 secretion by approximately 6-fold with $20 \mu \mathrm{M}$ of the inhibitor. 


\section{References}

1. Society AC. Cancer Facts and Figures 2017. In: American Cancer Society I, editor. Atlanta2017. p. 1-56.

2. Society AC. Breast Cancer Facts and Figures 2015-2016. Atlanta: American Cancer Society, Inc; 2016.

3. Park YM, White AJ, Nichols HB, O'Brien KM, Weinberg CR, Sandler DP. The association between metabolic health, obesity phenotype and the risk of breast cancer. Int J Cancer. 2017;140(12):2657-66. Epub 2017/03/28. doi: 10.1002/ijc.30684. PubMed PMID: 28268252; PMCID: PMC5406245.

4. Crespi E, Bottai G, Santarpia L. Role of inflammation in obesity-related breast cancer. Curr Opin Pharmacol. 2016;31:114-22. Epub 2016/11/24. doi: 10.1016/j.coph.2016.11.004. PubMed PMID: 27889687.

5. Deng T, Lyon CJ, Bergin S, Caligiuri MA, Hsueh WA. Obesity, Inflammation, and Cancer. Annu Rev Pathol. 2016;11:421-49. doi: 10.1146/annurev-pathol-012615044359. PubMed PMID: 27193454.

6. Iyengar NM, Gucalp A, Dannenberg AJ, Hudis CA. Obesity and Cancer Mechanisms: Tumor Microenvironment and Inflammation. J Clin Oncol. 2016;34(35):4270-6. Epub 2016/11/07. doi: 10.1200/JCO.2016.67.4283. PubMed PMID: 27903155.

7. Zahid H, Simpson ER, Brown KA. Inflammation, dysregulated metabolism and aromatase in obesity and breast cancer. Curr Opin Pharmacol. 2016;31:90-6. Epub 2016/11/19. doi: 10.1016/j.coph.2016.11.003. PubMed PMID: 27875786.

8. Khan S, Shukla S, Sinha S, Meeran SM. Role of adipokines and cytokines in obesity-associated breast cancer: therapeutic targets. Cytokine Growth Factor Rev. 2013;24(6):503-13. Epub 2013/10/21. doi: 10.1016/j.cytogfr.2013.10.001. PubMed PMID: 24210902.

9. Tawara K, Oxford JT, Jorcyk CL. Clinical significance of interleukin (IL)-6 in cancer metastasis to bone: potential of anti-IL-6 therapies. Cancer Manag Res. 2011;3:177-89. Epub 2011/05/18. doi: 10.2147/CMR.S18101. PubMed PMID: $21625400 ;$ PMCID: PMC3101113.

10. Angevin E, Tabernero J, Elez E, Cohen SJ, Bahleda R, van Laethem JL, Ottensmeier C, Lopez-Martin JA, Clive S, Joly F, Ray-Coquard I, Dirix L, Machiels JP, Steven N, Reddy M, Hall B, Puchalski TA, Bandekar R, van de Velde H, Tromp B, Vermeulen J, Kurzrock R. A phase I/II, multiple-dose, dose-escalation study of siltuximab, an anti-interleukin-6 monoclonal antibody, in patients with advanced solid tumors. Clin Cancer Res. 2014;20(8):2192-204. Epub 2014/02/21. doi: 10.1158/10780432.CCR-13-2200. PubMed PMID: 24563479.

11. Hudes G, Tagawa ST, Whang YE, Qi M, Qin X, Puchalski TA, Reddy M, Cornfeld M, Eisenberger M. A phase 1 study of a chimeric monoclonal antibody against interleukin-6, siltuximab, combined with docetaxel in patients with metastatic castrationresistant prostate cancer. Invest New Drugs. 2013;31(3):669-76. Epub 2012/07/25. doi: 10.1007/s10637-012-9857-z. PubMed PMID: 22828917.

12. Silver JS, Hunter CA. gp130 at the nexus of inflammation, autoimmunity, and cancer. J Leukoc Biol. 2010;88(6):1145-56. Epub 2010/07/07. doi: 10.1189/jlb.0410217. PubMed PMID: 20610800; PMCID: PMC2996896. 
13. Boulanger MJ, Garcia KC. Shared cytokine signaling receptors: structural insights from the gp130 system. Adv Protein Chem. 2004;68:107-46. doi: 10.1016/s00653233(04)68004-1. PubMed PMID: 15500860.

14. Heo TH, Wahler J, Suh N. Potential therapeutic implications of IL-6/IL6R/gp130-targeting agents in breast cancer. Oncotarget. 2016;7(13):15460-73. doi: 10.18632/oncotarget.7102. PubMed PMID: 26840088; PMCID: PMC4941253. 15. Smigiel JM, Parameswaran N, Jackson MW. Potent EMT and CSC Phenotypes Are Induced By Oncostatin-M in Pancreatic Cancer. Mol Cancer Res. 2017;15(4):47888. Epub 2017/01/04. doi: 10.1158/1541-7786.MCR-16-0337. PubMed PMID: 28053127; PMCID: PMC5380554.

16. Guihard P, Boutet MA, Brounais-Le Royer B, Gamblin AL, Amiaud J, Renaud A, Berreur M, Rédini F, Heymann D, Layrolle P, Blanchard F. Oncostatin m, an inflammatory cytokine produced by macrophages, supports intramembranous bone healing in a mouse model of tibia injury. Am J Pathol. 2015;185(3):765-75. Epub 2015/01/02. doi: 10.1016/j.ajpath.2014.11.008. PubMed PMID: 25559270.

17. Caffarel MM, Coleman N. Oncostatin M receptor is a novel therapeutic target in cervical squamous cell carcinoma. J Pathol. 2014;232(4):386-90. doi: 10.1002/path.4305. PubMed PMID: 24659184; PMCID: PMC4260121.

18. Bolin C, Tawara K, Sutherland C, Redshaw J, Aranda P, Moselhy J, Anderson R, Jorcyk CL. Oncostatin $\mathrm{m}$ promotes mammary tumor metastasis to bone and osteolytic bone degradation. Genes Cancer. 2012;3(2):117-30. doi: 10.1177/1947601912458284. PubMed PMID: 23050044; PMCID: PMC3463924.

19. Junk DJ, Bryson BL, Smigiel JM, Parameswaran N, Bartel CA, Jackson MW. Oncostatin M promotes cancer cell plasticity through cooperative STAT3-SMAD3 signaling. Oncogene. 2017. Epub 2017/03/13. doi: 10.1038/onc.2017.33. PubMed PMID: 28288136.

20. Jorcyk CL, Holzer RG, Ryan RE. Oncostatin M induces cell detachment and enhances the metastatic capacity of T-47D human breast carcinoma cells. Cytokine. 2006;33(6):323-36. doi: DOI: 10.1016/j.cyto.2006.03.004.

21. Lapeire L, Hendrix A, Lambein K, Van Bockstal M, Braems G, Van Den Broecke R, Limame R, Mestdagh P, Vandesompele J, Vanhove C, Maynard D, Lehuédé C, Muller C, Valet P, Gespach CP, Bracke M, Cocquyt V, Denys H, De Wever O. Cancerassociated adipose tissue promotes breast cancer progression by paracrine oncostatin $\mathrm{M}$ and Jak/STAT3 signaling. Cancer Res. 2014;74(23):6806-19. Epub 2014/09/24. doi: 10.1158/0008-5472.CAN-14-0160. PubMed PMID: 25252914.

22. Smith DA, Kiba A, Zong Y, Witte ON. Interleukin-6 and oncostatin-M synergize with the PI3K/AKT pathway to promote aggressive prostate malignancy in mouse and human tissues. Mol Cancer Res. 2013;11(10):1159-65. doi: 10.1158/1541-7786.MCR-130238. PubMed PMID: 23867565; PMCID: PMC3800471.

23. Fossey SL, Bear MD, Kisseberth WC, Pennell M, London CA. Oncostatin M promotes STAT3 activation, VEGF production, and invasion in osteosarcoma cell lines. BMC Cancer. 2011;11:125. doi: 1471-2407-11-125 [pii] 10.1186/1471-2407-11-125. PubMed PMID: 21481226; PMCID: PMC3079692. 24. Li C, Ahlborn TE, Kraemer FB, Liu J. Oncostatin M-induced growth inhibition and morphological changes of MDA-MB231 breast cancer cells are abolished by 
blocking the MEK/ERK signaling pathway. Breast Cancer Res Treat. 2001;66(2):111-21. PubMed PMID: 11437097.

25. Rowan AD, Koshy PJ, Shingleton WD, Degnan BA, Heath JK, Vernallis AB, Spaull JR, Life PF, Hudson K, Cawston TE. Synergistic effects of glycoprotein 130 binding cytokines in combination with interleukin-1 on cartilage collagen breakdown. Arthritis Rheum. 2001;44(7):1620-32. doi: 10.1002/1529-0131(200107)44:7<1620::AIDART285>3.0.CO;2-B. PubMed PMID: 11465713.

26. Hui W, Rowan AD, Richards CD, Cawston TE. Oncostatin M in combination with tumor necrosis factor alpha induces cartilage damage and matrix metalloproteinase expression in vitro and in vivo. Arthritis Rheum. 2003;48(12):3404-18. doi:

10.1002/art.11333. PubMed PMID: 14673992.

27. Barksby HE, Hui W, Wappler I, Peters HH, Milner JM, Richards CD, Cawston TE, Rowan AD. Interleukin-1 in combination with oncostatin M up-regulates multiple genes in chondrocytes: implications for cartilage destruction and repair. Arthritis Rheum. 2006;54(2):540-50. doi: 10.1002/art.21574. PubMed PMID: 16447230.

28. Repovic P, Fears CY, Gladson CL, Benveniste EN. Oncostatin-M induction of vascular endothelial growth factor expression in astroglioma cells. Oncogene. 2003;22(50):8117-24. doi: 10.1038/sj.onc.1206922. PubMed PMID: 14603252. 29. Apte RN, Dotan S, Elkabets M, White MR, Reich E, Carmi Y, Song X, Dvozkin T, Krelin Y, Voronov E. The involvement of IL-1 in tumorigenesis, tumor invasiveness, metastasis and tumor-host interactions. Cancer Metastasis Rev. 2006;25(3):387-408. doi: 10.1007/s10555-006-9004-4. PubMed PMID: 17043764.

30. Voronov E, Dotan S, Krelin Y, Song X, Elkabets M, Carmi Y, Rider P, Idan Cohen, Romzova M, Kaplanov I, Apte RN. Unique Versus Redundant Functions of IL$1 \alpha$ and IL-1 $\beta$ in the Tumor Microenvironment. Front Immunol. 2013;4:177. Epub 2013/07/08. doi: 10.3389/fimmu.2013.00177. PubMed PMID: 23847618; PMCID: PMC3703603.

31. Kwon G, Corbett JA, Rodi CP, Sullivan P, McDaniel ML. Interleukin-1 betainduced nitric oxide synthase expression by rat pancreatic beta-cells: evidence for the involvement of nuclear factor kappa B in the signaling mechanism. Endocrinology. 1995;136(11):4790-5. doi: 10.1210/endo.136.11.7588208. PubMed PMID: 7588208.

32. Raymond L, Eck S, Mollmark J, Hays E, Tomek I, Kantor S, Elliott S, Vincenti M. Interleukin-1 beta induction of matrix metalloproteinase-1 transcription in chondrocytes requires ERK-dependent activation of CCAAT enhancer-binding proteinbeta. J Cell Physiol. 2006;207(3):683-8. doi: 10.1002/jcp.20608. PubMed PMID: 16453302.

33. Mon NN, Senga T, Ito S. Interleukin-1 $\beta$ activates focal adhesion kinase and Src to induce matrix metalloproteinase-9 production and invasion of MCF-7 breast cancer cells. Oncol Lett. 2017;13(2):955-60. Epub 2016/12/20. doi: 10.3892/ol.2016.5521. PubMed PMID: 28356984; PMCID: PMC5351262.

34. Holen I, Lefley DV, Francis SE, Rennicks S, Bradbury S, Coleman RE, Ottewell P. IL-1 drives breast cancer growth and bone metastasis in vivo. Oncotarget. 2016;7(46):75571-84. doi: 10.18632/oncotarget.12289. PubMed PMID: 27765923; PMCID: PMC5342762. 
35. Dinarello CA. Why not treat human cancer with interleukin-1 blockade? Cancer Metastasis Rev. 2010;29(2):317-29. doi: 10.1007/s10555-010-9229-0. PubMed PMID: 20422276 ; PMCID: PMC2865633.

36. Lubberink M, Golla SS, Jonasson M, Rubin K, Glimelius B, Sörensen J, Nygren P. (15)O-Water PET Study of the Effect of Imatinib, a Selective Platelet-Derived Growth Factor Receptor Inhibitor, Versus Anakinra, an IL-1R Antagonist, on Water-Perfusable Tissue Fraction in Colorectal Cancer Metastases. J Nucl Med. 2015;56(8):1144-9. Epub 2015/06/11. doi: 10.2967/jnumed.114.151894. PubMed PMID: 26069310.

37. M.D. Anderson Cancer Center: Anakinra or Denosumab and Everolimus in Advanced CancerDecember 2016; Identification No. NCT01624766.

38. Meseure D, Vacher S, Drak Alsibai K, Trassard M, Nicolas A, Leclere R, Lerebours F, Guinebretiere JM, Marangoni E, Lidereau R, Bieche I. Biopathological Significance of TLR9 Expression in Cancer Cells and Tumor Microenvironment Across Invasive Breast Carcinomas Subtypes. Cancer Microenviron. 2016;9(2-3):107-18. Epub 2016/07/09. doi: 10.1007/s 12307-016-0186-1. PubMed PMID: 27392414; PMCID: PMC5264659.

39. Lehmann BD, Bauer JA, Chen X, Sanders ME, Chakravarthy AB, Shyr Y, Pietenpol JA. Identification of human triple-negative breast cancer subtypes and preclinical models for selection of targeted therapies. J Clin Invest. 2011;121(7):2750-67. doi: 10.1172/JCI45014. PubMed PMID: 21633166; PMCID: PMC3127435.

40. Kohler BA, Sherman RL, Howlader N, Jemal A, Ryerson AB, Henry KA, Boscoe FP, Cronin KA, Lake A, Noone AM, Henley SJ, Eheman CR, Anderson RN, Penberthy L. Annual Report to the Nation on the Status of Cancer, 1975-2011, Featuring Incidence of Breast Cancer Subtypes by Race/Ethnicity, Poverty, and State. J Natl Cancer Inst. 2015;107(6):djv048. Epub 2015/03/30. doi: 10.1093/jnci/djv048. PubMed PMID: 25825511; PMCID: PMC4603551.

41. Hon JD, Singh B, Sahin A, Du G, Wang J, Wang VY, Deng FM, Zhang DY, Monaco ME, Lee P. Breast cancer molecular subtypes: from TNBC to QNBC. Am J Cancer Res. 2016;6(9):1864-72. Epub 2016/09/01. PubMed PMID: 27725895; PMCID: PMC5043099.

42. West NR, Murphy LC, Watson PH. Oncostatin M suppresses oestrogen receptor$\alpha$ expression and is associated with poor outcome in human breast cancer. Endocr Relat Cancer. 2012;19(2):181-95. doi: 10.1530/erc-11-0326. PubMed PMID: 22267707. 43. West NR, Murray JI, Watson PH. Oncostatin-M promotes phenotypic changes associated with mesenchymal and stem cell-like differentiation in breast cancer. Oncogene. 2014;33(12):1485-94. doi: 10.1038/onc.2013.105. PubMed PMID: 23584474. 44. Bottai G, Diao L, Baggerly KA, Paladini L, Győrffy B, Raschioni C, Pusztai L, Calin GA, Santarpia L. Integrated MicroRNA-mRNA Profiling Identifies Oncostatin M as a Marker of Mesenchymal-Like ER-Negative/HER2-Negative Breast Cancer. Int J Mol Sci. 2017;18(1). Epub 2017/01/19. doi: 10.3390/ijms18010194. PubMed PMID: 28106823 ; PMCID: PMC5297825.

45. Curtis C, Shah SP, Chin SF, Turashvili G, Rueda OM, Dunning MJ, Speed D, Lynch AG, Samarajiwa S, Yuan Y, Gräf S, Ha G, Haffari G, Bashashati A, Russell R, McKinney S, Langerød A, Green A, Provenzano E, Wishart G, Pinder S, Watson P, Markowetz F, Murphy L, Ellis I, Purushotham A, Børresen-Dale AL, Brenton JD, Tavaré S, Caldas C, Aparicio S, Group M. The genomic and transcriptomic architecture of 2,000 
breast tumours reveals novel subgroups. Nature. 2012;486(7403):346-52. doi: 10.1038/nature10983. PubMed PMID: 22522925; PMCID: PMC3440846.

46. Finak G, Bertos N, Pepin F, Sadekova S, Souleimanova M, Zhao H, Chen H, Omeroglu G, Meterissian S, Omeroglu A, Hallett M, Park M. Stromal gene expression predicts clinical outcome in breast cancer. Nat Med. 2008;14(5):518-27. Epub 2008/04/27. doi: 10.1038/nm1764. PubMed PMID: 18438415.

47. Sullivan NJ, Sasser AK, Axel AE, Vesuna F, Raman V, Ramirez N, Oberyszyn TM, Hall BM. Interleukin-6 induces an epithelial-mesenchymal transition phenotype in human breast cancer cells. Oncogene. 2009;28(33):2940-7. doi: 10.1038/onc.2009.180. PubMed PMID: 19581928.

48. Walter M, Liang S, Ghosh S, Hornsby PJ, Li R. Interleukin 6 secreted from adipose stromal cells promotes migration and invasion of breast cancer cells. Oncogene. 2009;28(30):2745-55. doi: 10.1038/onc.2009.130. PubMed PMID: 19483720; PMCID: 2806057.

49. Lin S, Gan Z, Han K, Yao Y, Min D. Interleukin-6 as a prognostic marker for breast cancer: a meta-analysis. Tumori. 2015;101(5):535-41. doi: 10.5301/tj.5000357. PubMed PMID: 26045123.

50. Holzer RG, Ryan RE, Tommack M, Schlekeway E, Jorcyk CL. Oncostatin M stimulates the detachment of a reservoir of invasive mammary carcinoma cells: role of cyclooxygenase-2. Clin Exp Metastasis. 2004;21(2):167-76. PubMed PMID: 15168734. 51. Queen MM, Ryan RE, Holzer RG, Keller-Peck CR, Jorcyk CL. Breast cancer cells stimulate neutrophils to produce oncostatin M: Potential implications for tumor progression. Cancer Research. 2005;65(19):8896-904. doi: 10.1158/0008-5472.can-051734. PubMed PMID: ISI:000232199400043.

52. Salgado R, Junius S, Benoy I, Van Dam P, Vermeulen P, Van Marck E, Huget P, Dirix LY. Circulating interleukin-6 predicts survival in patients with metastatic breast cancer. Int J Cancer. 2003;103(5):642-6. doi: 10.1002/ijc.10833. PubMed PMID: 12494472.

53. Ben QW, Zhao Z, Ge SF, Zhou J, Yuan F, Yuan YZ. Circulating levels of periostin may help identify patients with more aggressive colorectal cancer. Int J Oncol. 2009;34(3):821-8. PubMed PMID: 19212687.

54. Bilgiç CI, Tez M. Serum VEGF levels in gastric cancer patients: correlation with clinicopathological parameters. Turk J Med Sci. 2015;45(1):112-7. PubMed PMID: 25790539.

55. Yoon S, Woo SU, Kang JH, Kim K, Shin HJ, Gwak HS, Park S, Chwae YJ. NF$\kappa \mathrm{B}$ and STAT3 cooperatively induce IL6 in starved cancer cells. Oncogene.

2012;31(29):3467-81. Epub 2011/11/21. doi: 10.1038/onc.2011.517. PubMed PMID: 22105366.

56. Le Goff B, Singbrant S, Tonkin BA, Martin TJ, Romas E, Sims NA, Walsh NC. Oncostatin $M$ acting via OSMR, augments the actions of IL-1 and TNF in synovial fibroblasts. Cytokine. 2014;68(2):101-9. Epub 2014/04/22. doi: 10.1016/j.cyto.2014.04.001. PubMed PMID: 24767864.

57. Oh K, Lee OY, Park Y, Seo MW, Lee DS. IL-1 $\beta$ induces IL-6 production and increases invasiveness and estrogen-independent growth in a TG2-dependent manner in human breast cancer cells. BMC Cancer. 2016;16(1):724. Epub 2016/09/08. doi: 10.1186/s12885-016-2746-7. PubMed PMID: 27609180; PMCID: PMC5017052. 
58. Gangadhara S, Barrett-Lee P, Nicholson RI, Hiscox S. Pro-metastatic tumorstroma interactions in breast cancer. Future Oncol. 2012;8(11):1427-42. doi:

10.2217/fon.12.134. PubMed PMID: 23148616.

59. Han SS, Yun H, Son DJ, Tompkins VS, Peng L, Chung ST, Kim JS, Park ES, Janz S. NF-kappaB/STAT3/PI3K signaling crosstalk in iMyc E mu B lymphoma. Mol Cancer. 2010;9:97. Epub 2010/04/30. doi: 10.1186/1476-4598-9-97. PubMed PMID: 20433747; PMCID: PMC2876994.

60. Liu H, Liu K, Bodenner DL. Estrogen receptor inhibits interleukin-6 gene expression by disruption of nuclear factor kappaB transactivation. Cytokine. 2005;31(4):251-7. doi: 10.1016/j.cyto.2004.12.008. PubMed PMID: 16043358.

61. Nakamura K, Smyth MJ. Targeting cancer-related inflammation in the era of immunotherapy. Immunol Cell Biol. 2017;95(4):325-32. Epub 2017/01/10. doi: 10.1038/icb.2016.126. PubMed PMID: 27999432.

62. Hurst SM, McLoughlin RM, Monslow J, Owens S, Morgan L, Fuller GM, Topley N, Jones SA. Secretion of oncostatin M by infiltrating neutrophils: regulation of IL-6 and chemokine expression in human mesothelial cells. J Immunol. 2002;169(9):5244-51. PubMed PMID: 12391243.

63. Ryan RE, Martin B, Mellor L, Jacob RB, Tawara K, McDougal OM, Oxford JT, Jorcyk CL. Oncostatin M binds to extracellular matrix in a bioactive conformation: implications for inflammation and metastasis. Cytokine. 2015;72(1):71-85. doi: 10.1016/j.cyto.2014.11.007. PubMed PMID: 25622278; PMCID: PMC4328881.

64. Casneuf T, Axel AE, King P, Alvarez JD, Werbeck JL, Verhulst T, Verstraeten K, Hall BM, Sasser AK. Interleukin-6 is a potential therapeutic target in interleukin-6 dependent, estrogen receptor- $\alpha$-positive breast cancer. Breast Cancer (Dove Med Press). 2016;8:13-27. Epub 2016/02/03. doi: 10.2147/BCTT.S92414. PubMed PMID: 26893580; PMCID: PMC4745841.

65. Sasser AK, Sullivan NJ, Studebaker AW, Hendey LF, Axel AE, Hall BM. Interleukin-6 is a potent growth factor for ER-alpha-positive human breast cancer. FASEB J. 2007;21(13):3763-70. Epub 2007/06/22. doi: 10.1096/fj.07-8832com. PubMed PMID: 17586727.

66. Asgeirsson KS, Olafsdóttir K, Jónasson JG, Ogmundsdóttir HM. The effects of IL-6 on cell adhesion and e-cadherin expression in breast cancer. Cytokine. 1998;10(9):720-8. doi: 10.1006/cyto.1998.0349. PubMed PMID: 9770334.

67. Badache A, Hynes NE. Interleukin 6 inhibits proliferation and, in cooperation with an epidermal growth factor receptor autocrine loop, increases migration of T47D breast cancer cells. Cancer Res. 2001;61(1):383-91. PubMed PMID: 11196191. 68. Heinrich PC, Behrmann I, Haan S, Hermanns HM, Müller-Newen G, Schaper F. Principles of interleukin (IL)-6-type cytokine signalling and its regulation. Biochem J. 2003;374(Pt 1):1-20. doi: 10.1042/bj20030407. PubMed PMID: 12773095; PMCID: PMC1223585.

69. Galeotti C, Tran TA, Franchi-Abella S, Fabre M, Pariente D, Koné-Paut I. IL1RA agonist (anakinra) in the treatment of multifocal castleman disease: case report. J Pediatr Hematol Oncol. 2008;30(12):920-4. doi: 10.1097/MPH.0b013e31818ab31f. PubMed PMID: 19131781.

70. El-Osta H, Janku F, Kurzrock R. Successful treatment of Castleman's disease with interleukin-1 receptor antagonist (Anakinra). Mol Cancer Ther. 2010;9(6):1485-8. Epub 
2010/05/25. doi: 10.1158/1535-7163.MCT-10-0156. PubMed PMID: 20501803; PMCID: PMC3108331.

71. National Cancer Institute (NCI): Anakinra in Treating Patients With Metastatic Cancer Expressing the Interleukin-1 GeneApril 29, 2015;NCT00072111. 72. Baylor Research Institute: Gemcitabine, Nab-Paclitaxel, Cisplatin and Anakinra Treatment on Patients With Pancreatic CancerMarch 16, 2016;NCT02550327.

73. Galien R, Garcia T. Estrogen receptor impairs interleukin-6 expression by preventing protein binding on the NF-kappaB site. Nucleic Acids Res. 1997;25(12):24249. PubMed PMID: 9171095; PMCID: PMC146754.

74. Yoshida Y, Kumar A, Koyama Y, Peng H, Arman A, Boch JA, Auron PE. Interleukin 1 activates STAT3/nuclear factor-kappaB cross-talk via a unique TRAF6- and p65-dependent mechanism. J Biol Chem. 2004;279(3):1768-76. Epub 2003/10/30. doi: 10.1074/jbc.M311498200. PubMed PMID: 14593105.

75. Natarajan K, Singh S, Burke TR, Grunberger D, Aggarwal BB. Caffeic acid phenethyl ester is a potent and specific inhibitor of activation of nuclear transcription factor NF-kappa B. Proc Natl Acad Sci U S A. 1996;93(17):9090-5. PubMed PMID: 8799159; PMCID: PMC38600.

76. Feldman I, Feldman GM, Mobarak C, Dunkelberg JC, Leslie KK. Identification of proteins within the nuclear factor-kappa $\mathrm{B}$ transcriptional complex including estrogen receptor-alpha. Am J Obstet Gynecol. 2007;196(4):394.e1-11; discussion .e11-3. doi: 10.1016/j.ajog.2006.12.033. PubMed PMID: 17403432; PMCID: PMC2175481. 